\title{
Morphological and molecular adaptation of Aspergillus niger to simulated spaceflight and Mars-like conditions
}

\section{Dissertation}

\author{
to obtain the Ph.D. degree \\ at the Faculty of Biology and Psychology \\ at the Georg-August-University Göttingen, Germany
}

in frame of the doctoral program

Georg-August University School of Science (GAUSS) - Biology

submitted by

\section{Marta Filipa Barros Cortesão Rocha Fernandes}

Born in Porto, Portugal on $17^{\text {th }}$ March 1993

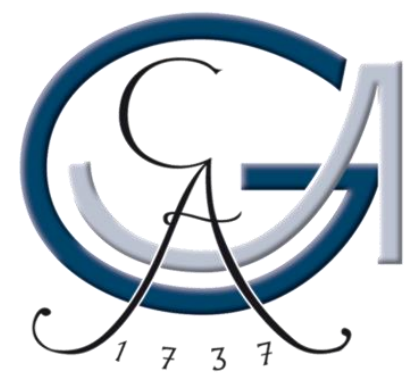

Göttingen, May 2021 


\section{Thesis Advisory Committee}

\section{Prof. Dr. Stefanie Pöggeler}

Department of General Microbiology, Institute of Microbiology and Genetics, Georg-August University of Göttingen

\section{Prof. Dr. Ralf Möller}

Aerospace Microbiology Research Group, Radiation Biology Department, Institute of Aerospace Medicine, German Aerospace Center (DLR), Cologne

University of Applied Sciences Bonn-Rhein-Sieg (BRSU), Department of Natural Sciences, Rheinbach, Germany

\section{Prof. Dr. Michael Hoppert}

Department of General Microbiology, Institute of Microbiology and Genetics, Georg August University of Göttingen

\section{Members of the examination board}

\section{First reviewer:}

\section{Prof. Dr. Stefanie Pöggeler}

Department of General Microbiology, Institute of Microbiology and Genetics, Georg-August University of Göttingen

\section{Second reviewer:}

\section{PD Dr. Michael Hoppert}

Department of General Microbiology, Institute of Microbiology and Genetics, Georg-August University of Göttingen

\section{Additional members of the examination board:}

\section{Prof. Dr. Ralf Möller}

Aerospace Microbiology Research Group, Radiation Biology Department, Institute of Aerospace Medicine, German Aerospace Center (DLR), Cologne

University of Applied Sciences Bonn-Rhein-Sieg (BRSU), Department of Natural Sciences, Rheinbach, Germany

\section{Prof. Dr.-Ing. Vera Meyer}

Chair of Applied and Molecular Microbiology, Institute of Biotechnology, Technical University of Berlin (TU Berlin)

\section{PD Dr. Wilfried Kramer}

Department of Molecular Genetics, Institute of Microbiology and Genetics, Georg-August University of Göttingen

\section{Prof. Dr. Kai. Haimel}

Department of Molecular Microbiology and Genetics, Institute of Microbiology and Genetics, Georg-August University of Göttingen

Date of Disputation:

$17^{\text {th }}$ June 2021 


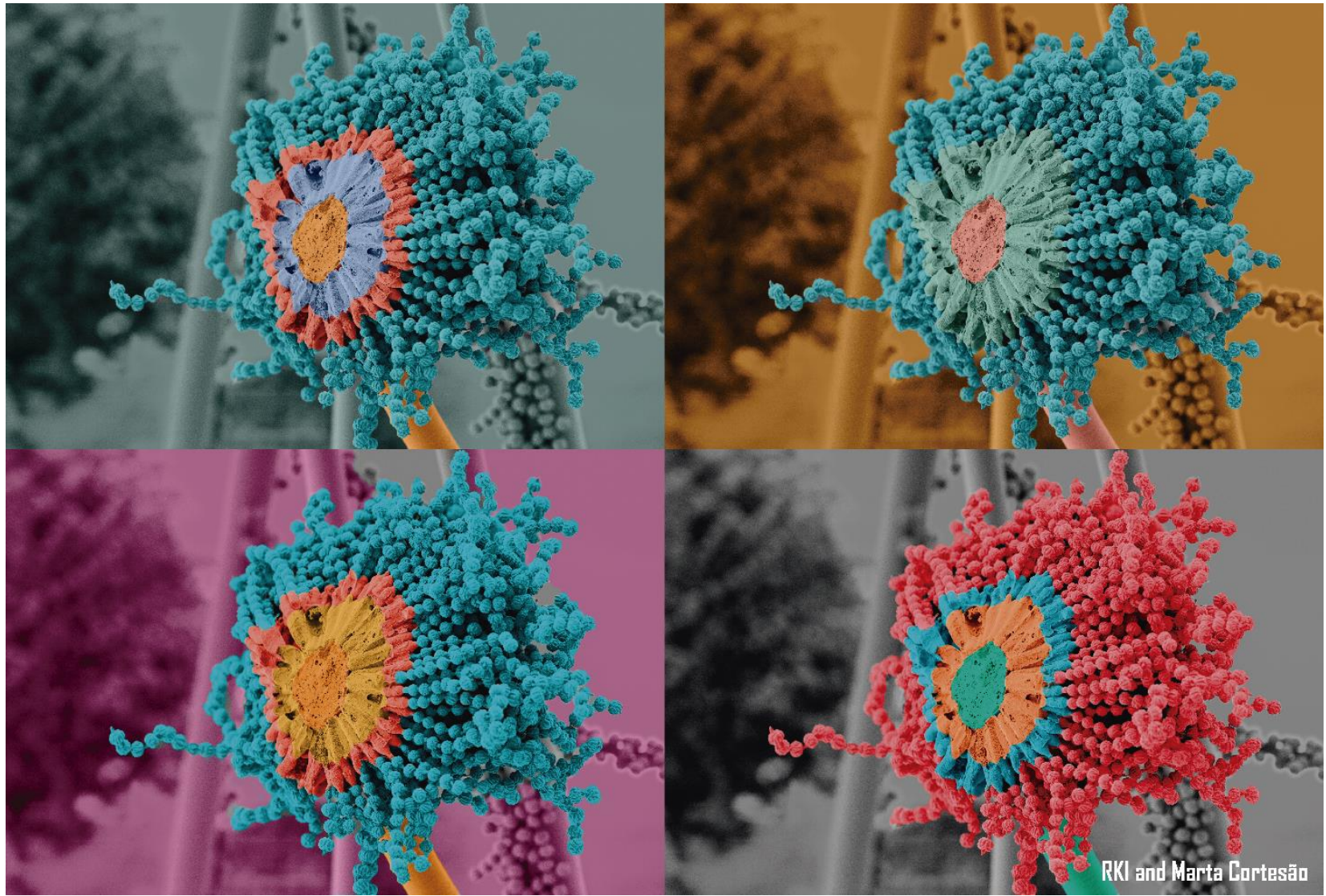

Aspergillus niger conidiophore seen via Scanning Electron Microscopy and edited in Adobe Photoshop.

- We are all made of Star stuff, we belong to the Universe - 


\section{Acknowledgements}

People have asked me why I came to Germany. The only reason was this: I wanted to become an Astrobiologist. I had no idea what was waiting for me in Cologne. It was hard in the beginning, to set in a new country, new language, a new lab, and new friends. It was also the first time having my own apartment. What an adventure these 4 years were. Now I know I have grown exponentially, and I have a lot of people to thank for that growth. To Felix, Sebastian, Andrea, Bahar, Aram, Rebecca, Jan, Nikea, Florian, Stella, Ina, Erika, Andrea, and Bentley, and everyone that has joined us in our group's cool and crazy spirit. Thank you for helping me feel at home, even though I am very far away. Thank you, Vera, Tabea, Lars, Sophia, Claudia, Charlie and Paul and all the others for welcoming in Berlin, for helping me in my fungal-existential questions and for making my PhD a thousand times better! Thank you, Prof. Pöggeler, Daniela, Gertrud and students, for welcoming me in Göttingen and GAUSS. Thank you, Prof. Dr. Commichau and Prof. Dr. Hoppert for acting as my doctoral supervisors. Thank you, Prof. Dr. Heimel and Prof. Dr. Kramer for accepting to participate in the examination board of my doctoral thesis. Thank you Luis, Phil, Zeena, Ryley, Tobias and Karen for welcoming me in CU Boulder, for the experiments, the experiences and the good times, I absolutely loved being there. Thank you Venkat, Parag, Snehit, Ada, Donatella, Cynthia, Nitin for welcoming me in at JPL and the city of Pasadena. Thank you, David and Sam, for being awesome and welcoming me at NASA Ames. Thank you, Akira and Hiro, for welcoming me in Chiba and for sharing with me your amazing Japanese culture. Thank you, Tiago, for helping me get through the hard times and teaching me to enjoy Germany. Thank you, Hector, for helping me stay motivated this final year, especially through Corona, and for helping me re-set life-goals and ambitions. Thank you, mom, sis, aunt, uncle and all my family for always supporting me, even when far away. Special thanks to you, mom, for being my biggest supporter, for all the rides to and from the airport, and for cooking my favourite foods whenever I come back to Porto! Thank you dad, for turning me into a space geek (it definitely showed in my career decision) - I know you would be proud, and I wish you could be here to celebrate with us. Most of all, thank you Ralf for being an exemplary mentor, for teaching me the tips and tricks of being a scientist and for trusting me and giving me the freedom to try, to fail, to succeed, but mainly, to learn and grow. These were four years of many conferences and presentations, networking meetings, networking dinners and networking lunches. Four years of many lab days, coffee breaks, lab cleaning and rearranging. Of many days in public transportation, either in train rides across Germany or on flight journeys across the world. Through all of this I have learned to be more of myself, to be a better person and to be a better scientist. I must confess I never expected to have such a great time during my PhD. I am looking forward to what's coming next!

- Marta Cortesão, May 2021 


\section{Affidavit}

Here I declare that my doctoral thesis entitled

"Morphological and molecular adaptation of Aspergillus niger to simulated spaceflight and Mars-like conditions"

has been written independently with no other sources and aids than quoted.

Signature

Marta Cortesão

Göttingen, June 30th 2021 


\section{Table of contents}

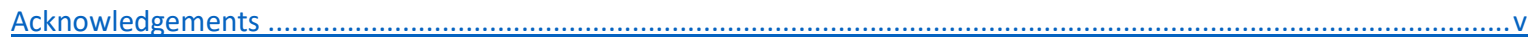

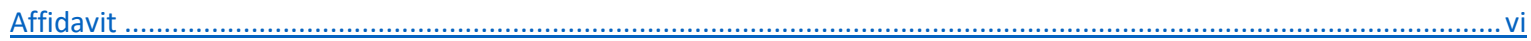

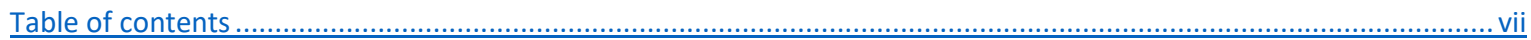

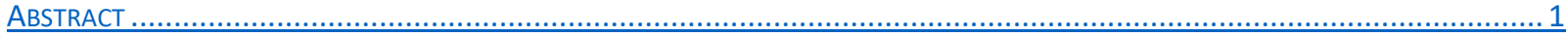

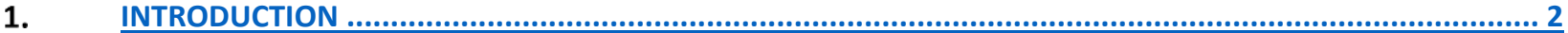

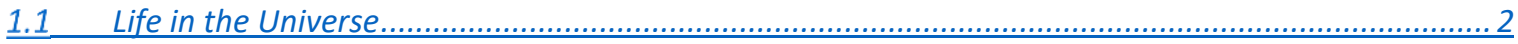

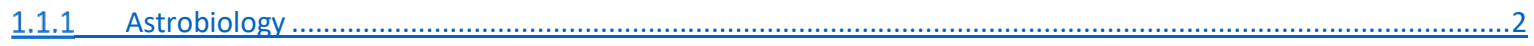

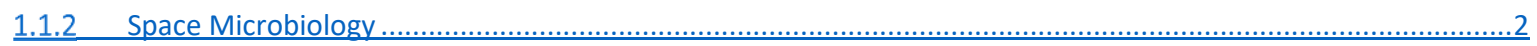

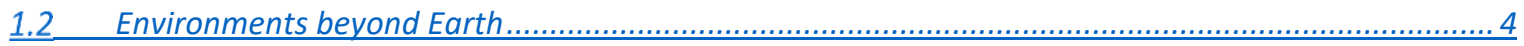

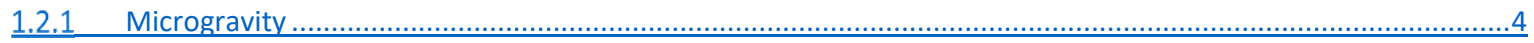

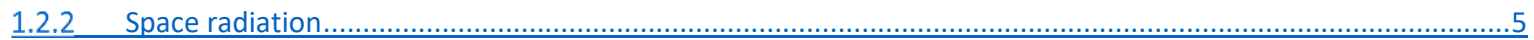

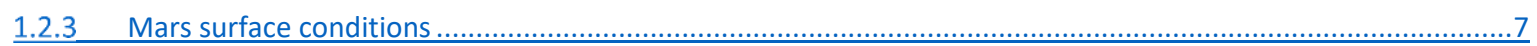

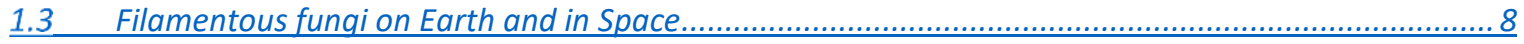

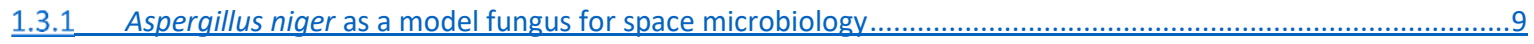

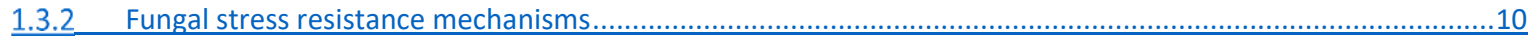

1.3.3 Real and simulated space experiments with filamentous fungi ...........................................................15

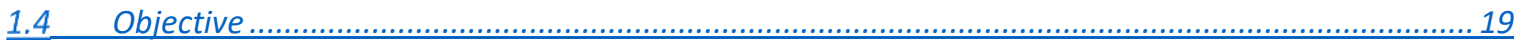

2. CHAPTER I: ASPERGILLUS NIGER SPORES ARE HIGHLY RESISTANT TO SPACE RADIATION..................... 20

3. CHAPTER II: MARSBOX: BACTERIAL AND FUNGAL ENDURANCE FROM A BALLOON-FLOWN ANALOG

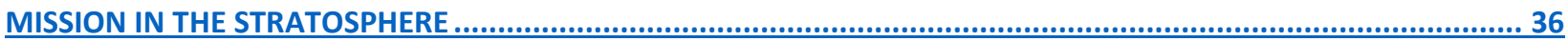

4. CHAPTER III: COLONY GROWTH AND BIOFILM FORMATION OF ASPERGILLUS NIGER UNDER SIMULATED

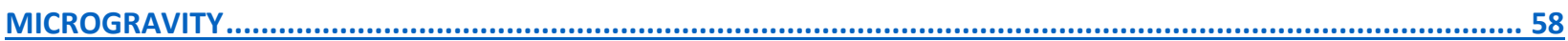

5. CHAPTER IV: DESIGN OF A SPACEFLIGHT BIOFILM EXPERIMENT AND CONTROLLING SPACEFLIGHT

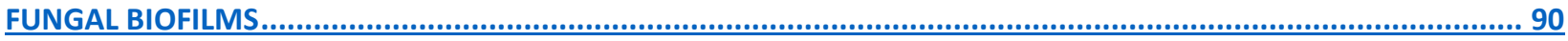

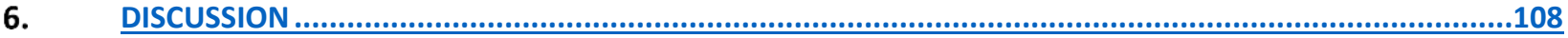

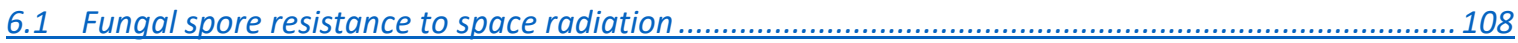

6.2 Fungal spore survival in a Mars-like environment......................................................... 109

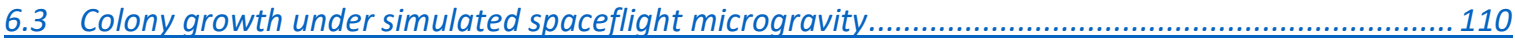

6.4 Penicillium rubens as model organism for a spaceflight experiment ....................................112

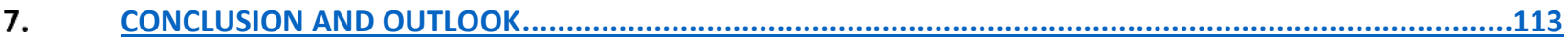

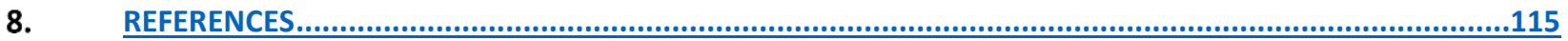

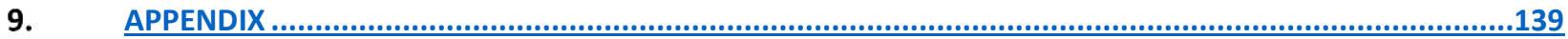




\section{Abstract}

The International Space Station (ISS) is an indoor-closed environment in Low Earth Orbit (LEO). Outside of the ISS, radiation is the most challenging factor outside. In turn, inside the ISS, spaceflight microgravity is the one factor that cannot be evaded. Aspergillus niger and Penicillium rubens are two common isolates of the ISS microbiota. As filamentous fungi, they form highly resistant airborne spores that can easily spread and colonize the spacecraft habitat. Fungi surface-associated growth (or biofilm formation), can biodegrade surfaces and clog life-support systems, and their spores can potentially infect the humans on board. In contrast, on Earth filamentous fungi play an important role in biotechnology, producing a widerange of compounds of interest, from food to antibiotics. Because of this, envisioned long-term spaceflight missions going far beyond low Earth orbit, to the Moon or Mars, will require an intensification of the fungal research, not only in relation to astronaut health and spacecraft safety, but also establishing opportunities for fungal-based biotechnology in space. Thus, this thesis aims to answer three main questions: i) can $A$. niger spores resist space radiation, and if yes, could they endure interplanetary space travel? ii) if brought to the surface of Mars, could $A$. niger spores survive the martian environment? and iii) how does simulated microgravity affect $A$. niger colony growth and biofilm formation? In total, four strains of $A$. niger were analyzed in this thesis: the industrial and highly pigmented wild-type strain (N402), a strain defective in pigmentation $(\triangle f w n A)$, a strain defective in DNA repair $(\Delta k u s A)$, and a strain defective in polar growth $(\triangle \operatorname{rac} A)$. To assess the level of resistance and survival limits of fungal spores in a long-term interplanetary mission scenario, $A$. niger spores were exposed to high radiation doses of $\mathrm{X}$-rays and cosmic radiation (helium- and iron-ions) and of UV-C radiation. Results show that wild-type spores of $A$. niger were able to withstand high doses of the all tested types of space radiation. This suggests that $A$. niger spores might endure space travel, when considering the radiation factor alone. To evaluate the survival of $A$. niger to Mars surface conditions, dried spores were launched in a stratospheric balloon mission called MARSBOx. Throughout the mission, $A$. niger spores were exposed to desiccation, simulated martian atmosphere and pressure, as well as to full UV-VIS radiation. Results revealed that the highly pigmented wild-type spores would survive in a Mars-like middle stratosphere environment with radiation exposure, even as a spore monolayer $\left(10^{6}\right.$ spores $\left./ \mathrm{ml}\right)$, i.e. with no self-shielding. Spore survival to space radiation and martian conditions suggest that current planetary protection guidelines should be revisited integrating the high resistance of fungal spores. Furthermore, $A$. niger colony growth and biofilm formation under simulated microgravity was investigated. Scanning Electron Microscopy (SEM) pictures reveal never-before seen ultrastructure of $A$. niger colonies and biofilms (i.e. vegetative mycelium embedded in extracellular matrix). Results reveal changes in biofilm thickness, spore production and dry biomass, suggesting an increased potential for $A$. niger to colonize spaceflight habitats. Lastly, $P$. rubens was proven as a model organism for a spaceflight biofilm experiment aboard the International Space Station. Overall, this thesis highlights the extraordinary resistance of fungal spores to extraterrestrial conditions and reveals their ability to cope with spaceflight microgravity. This advocates for future research that will enable better monitoring and controlling of fungal contaminations in space habitats, and that will help establish filamentous fungi as valuable companions of human space exploration. 


\section{Introduction}

\subsection{Life in the Universe}

\subsubsection{Astrobiology}

How has life originated and why? Is there is life beyond Earth? Will we ever find it? These are some of the oldest and most challenging questions of humankind. With the 1960s space age there was a need to understand how life adapts and survives in space. Not only humans, but also other organisms. With this, scientists such as Joshua Lederberg and Carl Sagan created the discipline of Exobiology (Lederberg, 1960). 30 years later, this is discipline became known as Astrobiology - the scientific field that studies the origin, evolution and distribution of life in the Universe (Soffen, 1997). Astrobiology is highly inter-disciplinary, requiring people from different scientific fields (e.g. biology, chemistry, geology, astronomy, physics and engineering) working side-by-side to unravel the mysteries of life in the Universe. Earth is, so far, the only planet we know that harbors life. Because of this, astrobiologists use what it's called "the Great Analogy", where Earth is the main (and only) example used to predict how life elsewhere could be possible. Given we only have ourselves to start with, astrobiologists extensively study planet Earth and its surrounding environment (our Solar System, our Galaxy), for clues on how life could have originated here. Active research in the Astrobiology field is being conducted world-wide. Both the NASA Astrobiology Roadmap (Des Marais et al., 2008) and the European Astrobiology Roadmap (Horneck et al., 2016) summarize guidelines, and focus areas for astrobiology. Today astrobiological research includes studying our own planet's extreme environments; life-detection missions on Mars; studying the composition of icy moons of our solar system, such as Enceladus (moon of Saturn), and Europa (moon of Jupiter); as well as the search for potentially habitable exoplanets (planets outside of our solar system) (Horneck et al., 2010). Another important astrobiological research area is the study of the limits of life and how terrestrial microorganisms survive and adapt to extreme extraterrestrial environments. To this research area we call Space Microbiology.

\subsubsection{Space Microbiology}

Space Microbiology is part of the research topic number 4 "Life and Habitability", of the European Astrobiology Roadmap (Horneck et al., 2016). It studies how terrestrial microorganisms survive and adapt to extreme environments, and it helps us understand the limits of life as we know it and whether or not there might be life on other planetary bodies. Extremophiles are "extreme-loving" microbes that are able to survive and grow in extreme conditions. They can not only provide valuable information on how life was on Early Earth, 4.5 billion years ago, but also whether or not modern terrestrial life could exist beyond Earth. Earth harbors many extreme environments, and some can be considered analogs for extraterrestrial environments. For instance, the cold McMurdo Dry Valleys in Antarctica, or the Atacama Desert in Chile, can be considered analog to Mars (Azua-Bustos et al., 2012; Van Ombergen et al., 2021), whereas deepsea hydrothermal vents are considered analog environments to the ocean world of Europa (moon of Jupiter) (Longo and Damer, 2020). The International Space Station (ISS) itself, established in 1998, acts as a valuable space microbiology laboratory. On the ISS, astronauts work as scientists, conducting microbiology experiments both inside and outside of the spacecraft (Horneck et al., 2010). Microbiology 
experiments aboard the International Space Station make up some of the most innovative and challenging research. However, space research is also met with several limitations. Costs, time, astronaut and instrument availability, microbial transport and stowage conditions, and space agency regulations are some of the most common limitations. This often results in a reduced flexibility of the experimental design, and reduced quality and quantity of the desired outcomes. To overcome these limitations, ground-based space simulation facilities allow the performance of space-relevant experiments, reducing the costs and increasing experimental flexibility. Several platforms are available world-wide and are able to provide functional simulations (more on section 1.2). When possible, space simulation experiments are validated by comparison with real space experiments.

How microbes tolerate and grow in extreme environments gives us knowledge on the cellular and molecular mechanisms behind their resistance. It can also inform us on current planetary protection policies regulated by The Committee on Space Research (COSPAR). COSPAR was created in 1958 with affiliations from countries involved in spaceflight activities (Conley, 2014). These policies aim to prevent cross-contamination between planetary bodies, and consist on cleaning and sterilization protocols as well as definition of thresholds limiting the amount of microbial burden (or bioburden) on a spacecraft surface. The policies, or guidelines, depend on the main objective of each space mission (COSPAR, 2020; Gradini et al., 2019; Regberg et al., 2020). Indeed, space microbiology research is in the forefront of science allowing for novel insights on how microbes could survive and adapt to extraterrestrial environments. Interestingly, space stations are scientific experiments in themselves. As indoor-closed habitats, monitoring microbial diversity is crucial to guarantee spacecraft safety and astronaut health (Ichijo et al., 2016; Mhatre et al., 2020; Mora et al., 2016; Yamaguchi et al., 2014). Microbial monitoring was done already in early space missions such as Apollo, Skylab, and Space Shuttle, where microbial samples were taken of the spacecraft, and were brought back to Earth to be isolated and characterized (Brockett and Ferguson, 1975; Castro et al., 2004; Pierson, 2001; Puleo et al., 1973). Today, state-of-the-art sequencing methods allow the characterization of the ISS's microbiome, detecting and identifying even non-culturable organisms (Be et al., 2017; Lang et al., 2017; Stahl-Rommel et al., 2021; Venkateswaran et al., 2014). The most common bacterial isolates aboard the ISS were spore-formers such as Bacillus spp. and Paenibacillus spp., and the most common fungal isolates were found to be Aspergillus and Penicillium spp., which are also sporeformers (Checinska et al., 2015). Interestingly, the former Russian space station Mir was known to welcome astronauts with a "strange smell", attributed to a diverse fungal flora. In fact, the high microbial burden was one of the main reasons why the Mir-station was terminated, given the risk to astronaut health and material integrity (Makimura et al., 2001). Currently there are several measures being taken aboard the ISS to monitor and control microbial contamination. For instance, astronauts are responsible for a thorough cleaning of the ISS, and air-filtration systems have HEPA-filters that are able to prevent microorganisms from further circulating the indoor habitat. Still, many microorganisms can go undetected and find their way to contaminate a wide range of spacecraft surfaces and systems. Unfortunately microbe-led material corrosion can affect metals, plastics and other surfaces (Beech et al., 2005; Gu et al., 1998; Klintworth et al., 1999; Videla and Herrera, 2005).

While on the one hand, controlling microbial contamination from negatively affecting life-support systems such as drinking water system, air-ventilation system, heating and electricity supply systems is a focus of crewed space exploration (Haines et al., 2019; Thornhill and Kumar, 2018; Vaishampayan and Grohmann, 2019). On the other hand, many research efforts look forward to bring such microorganisms into play to 
improve mentioned life-support systems. Due to their light weight and multi-purpose character, microorganisms can be of great help in space exploration when grown in a planned and controlled manner. Indeed, the use of microorganism-based technologies for in situ food production, water, air, and waste recovery are being investigated (Ilgrande et al., 2019; Soreanu et al., 2021; Wagner et al., 2016). For instance, extremophile applications in biotechnology, e.g. through extremoenzymes, have been a focus area (Gabani and Singh, 2013; Raddadi et al., 2015). With all of this, knowing how microorganisms adapt and grow in environments beyond Earth, will not only be essential to better understand the origin of life on Earth and establish the limits of life as we know it, but also to develop efficient space exploration strategies that help thrive the human presence in space.

\subsection{Environments beyond Earth}

\subsubsection{Microgravity}

On Earth, gravity of 1-g (or $9.81 \mathrm{~m} / \mathrm{s}^{2}$ ) has been the one constant along the history of evolution of life. Because of this, both flora and fauna have adapted to gravity on Earth (Najrana and Sanchez-Esteban, 2016), and so have all associated chemical, physical and biological processes. In turn, microgravity, also known as weightlessness, or zero $\mathrm{g}$, is considered when the gravitational regime is in lower than $1 \times 10^{-3}$ g. The International Space Station orbits the Earth at $400 \mathrm{~km}$ altitude (in low Earth orbit), and at a constant speed of $8 \mathrm{~km} /$ second, achieving a microgravity environment of $1 \times 10^{-4}$ to $10^{-6}$-g (Stenzel, 2016). Spaceflight microgravity is one of the most unique environments, affecting every living organism aboard the international space station. An average astronaut mission on the ISS lasts 6 months, during which the spaceflight environment affects the human body in numerous ways. A compromised immune system, shifts in body fluids, muscle and bone loss, are some consequences of exposure to microgravity, low dose radiation, changes in diet, and mission stress (e.g. sleep, schedule) (Bigley et al., 2019; Mann et al., 2019). Space microbiology experiments have found several spaceflight-induced changes in different microorganisms, such as increased pathogenicity, increased resistance towards antibiotics, differences in growth and changes in biofilm formation (Horneck et al., 2010; Huang et al., 2018; Kim et al., 2013; Leys et al., 2004; Nickerson et al., 2004; Ott et al., 2012; Rosenzweig et al., 2010, 2014; Senatore et al., 2018; Zea et al., 2017). In outer space, i.e. interplanetary space or deep space, microgravity reaches $1 \times 10^{-12} \mathrm{~g}$, which astronauts will experience for example on during the flight to the Moon, or Mars. Once at our interplanetary destination, partial gravity of the Moon (0.17-g) and Mars (0.38-g), will also affect biological processes of humans and microbes (Garshnek, 1994; Horneck et al., 2003; Kiss, 2014; Santomartino et al., 2020).

Knowing how life adapts to microgravity (and other gravitational regimes) will be important to assess both risks and opportunities of space exploration. Given that real microgravity experiments are both costly and of difficult access, studies have been performed using microgravity simulations. Different facilities and devices vary in quality of microgravity simulation, as well as in duration of exposure (Herranz et al., 2012; Kiss, 2014). Platforms such as drop towers or parabolic flights provide short-term high-quality microgravity, where samples are exposed in the time range of seconds (10 - 30 seconds) (Braun et al., 2002; Limbach et al., 2005). Moreover, sounding rockets can provide good quality microgravity environment in the time range of minutes (6 - $12 \mathrm{~min}$ ) (Hemmersbach et al., 2006; Kopp et al., 2018). 
Suborbital platforms such as re-entry capsules can provide both good quality microgravity $\left(10^{-5}-\mathrm{g}\right)$ and long-duration exposure (over 2 weeks), however they are very rare and cost-intensive (Cogoli, 1993; Gulimova et al., 2019; Pletser, 2004). In contrast, small devices, such as the Rotating Wall Vessel (RWV), the High Aspect Ration Vessel (HARV), the clinostat (also known as 2-D clinostat) (Walther et al., 2000), or the Random Positioning Machine (RPM) (also known as 3-D clinostat) (Borst and van Loon, 2008), are very cost-effective and can provide long-duration microgravity simulation in the range of days and weeks, depending on the model organism. These small devices provide a functional simulation of microgravity sharing the same principle: by continuously rotating the samples they reduce the gravity vector to close to zero, and consequently prevent particle sedimentation. In 2-D clinostats, microgravity is simulated by continuously rotating the samples around the horizontal axis. Here the best quality of microgravity is simulated at the very center of the rotation axis. Further from the center, shear forces occur and the gravitational force increases (van Loon et al., 2003, 2016). Notably, the 2-D clinostat has been recognized to provide better simulation of microgravity than HARV, given that HARV can only provide low shear modelled microgravity (Klaus, 2001). The 2-D clinostat can be set to be fast-rotating or slow-rotating, depending on the viscosity of the culture medium, to ensure the least amount of shear forces. Here, liquid cultures (low viscosity) would require a slow-clinorotation (10 - 25 revolutions per minute, or r.p.m), whereas solid media (agar) cultures (with high viscosity) would require fast-clinorotation (60-90 r.p.m). The clinorotation principle has been described as early as 1872 , where plants were rotated $0.1-0.03$ r.p.m (Newcombe, 1904), and has evolved to be an established scientific device for microgravity simulation. Today, different clinostat set-ups can carry various sample holders (petri-dish, slide-flask, pipettes, FEPbag, etc.), in both liquid or solid cultures (Brungs et al., 2015; Garschagen et al., 2019; Herranz et al., 2012; Liu et al., 2020).

\subsubsection{Space radiation}

On Earth, the magnetic field, ozone layer and atmosphere protect humans from heavy radiation exposure, preventing damages to our cells and DNA. Beyond Earth and into the depths of space, the environment becomes extreme. With vacuum, extreme temperatures and high doses of different types of radiation, outer space is the most extreme environment to life as we know it (Horneck et al., 2006). In space, protection from radiation is hard to achieve, and so radiation is considered the most challenging factor for space exploration (Chancellor et al., 2014). There are different qualities of space radiation (Figure 1). Nonionizing Ultraviolet (UV) radiation can be divided into three types, depending on the wavelength: UV-A (315 - $400 \mathrm{~nm}$ ) which is completely absorbed by the ozone layer and atmosphere; UV-B (280 - $315 \mathrm{~nm}$ ) which is mostly absorbed by the ozone layer; and UV-C $(100-280 \mathrm{~nm})$ which is not absorbed by the ozone layer and is the most damaging for cells. Besides UV radiation, our sun naturally emits ionizing X-rays. When there are solar particle events (or solar flares) these emit high doses of X-rays, up to $>5000 \mu \mathrm{Gy} / \mathrm{h}$ which are dangerous to the astronauts on board the ISS (Dachev et al., 2017). In addition, cosmic events such as supernova explosions or pulsars emit what is known as Galactic Cosmic Radiation (here termed cosmic rays). Cosmic rays consist mainly of protons ( $87 \%)$, helium ( $12 \%)$, and heavy ions ( $1 \%)$. The $1 \%$ heavy ions are considered highly $(\mathrm{H})$ charged (atomic number, $\mathrm{Z}$ ) and energy (E) particles (HZE) such as Iron (Fe) ions (Horneck et al., 2010). Cosmic rays with charges higher than $Z>2$ have energy high enough to pierce through space hardware shielding of $1 \mathrm{~mm}$ thick, and can pose a risk to organisms aboard 
(Horneck et al., 2010). Solar flares, and cosmic events can also emit Gamma rays, which are high-energy, short-wavelength electromagnetic waves produced as a result of the disintegration of radioactive atomic nuclei and in decay of subatomic particles. In 2016, cosmic ray exposure on the ISS was registered as an average of $286 \mu \mathrm{Gy} /$ day (Berger et al., 2017). These doses increase dramatically if we are considering interplanetary travel. For instance, on a 360-day round-trip to Mars yields a dose of $0.66 \pm 0.12 \mathrm{~Gy}$ (Zeitlin et al., 2013). It is important to note that acute radiation syndrome (followed by death) can be induced in humans at a dose of around 5 Gy (Cucinotta and Durante, 2006; Hellweg and Baumstark-Khan, 2007). Because UV-C greatly damages cells and biomolecules, monochromatic UV-C radiation (254 $\mathrm{nm}$ ) is commonly used on Earth for food sterilization, in hospital surface disinfection (Corrêa et al., 2017; Santos and de Castro, 2021), or in microbiology laboratories, for instance, in safety cabinets where the UV lamp is used for around $15 \mathrm{~min}$, reaching a UV dose of approximately $2000 \mathrm{~J} / \mathrm{m}^{2}$ (Begum et al., 2009). Also Xrays, gamma rays and electron-beam (e-beam) radiation have been extensively used in sterilization procedures of medical devices, pharmaceuticals and food (Harrell et al., 2018; Hasanain et al., 2014; Moosekian et al., 2012; Munir and Federighi, 2020; Ravindran and Jaiswal, 2019). Despite the damaging effects of radiation, many microorganisms are known to be able to resist outstandingly high doses.

Microbial radiation exposure experiments can be done both outside of the ISS and on Earth, using ground facilities. For instance, the German Aerospace Center (DLR), in Cologne, has so-called planetary simulation facilities, as an official part of ESA's space simulation platforms (Rabbow et al., 2016). Here, UV lamps and UV crosslinkers allow for easy and cost-effective exposure to monochromatic (e.g. $254 \mathrm{~nm}$ ) or polychromatic UV radiation (200-400 nm). Also X-ray systems are commercially available and enable the application of targeted doses to biological samples. Both UV and X-ray exposure can be specified by adjusting the dose rate (measured using a dosimeter) and regulating the height between the radiation source and the sample (Moeller et al., 2007). Exposure to heavy ions can be achieved with a heavy ion accelerator (Moeller et al., 2008).

\section{Space Radiation}

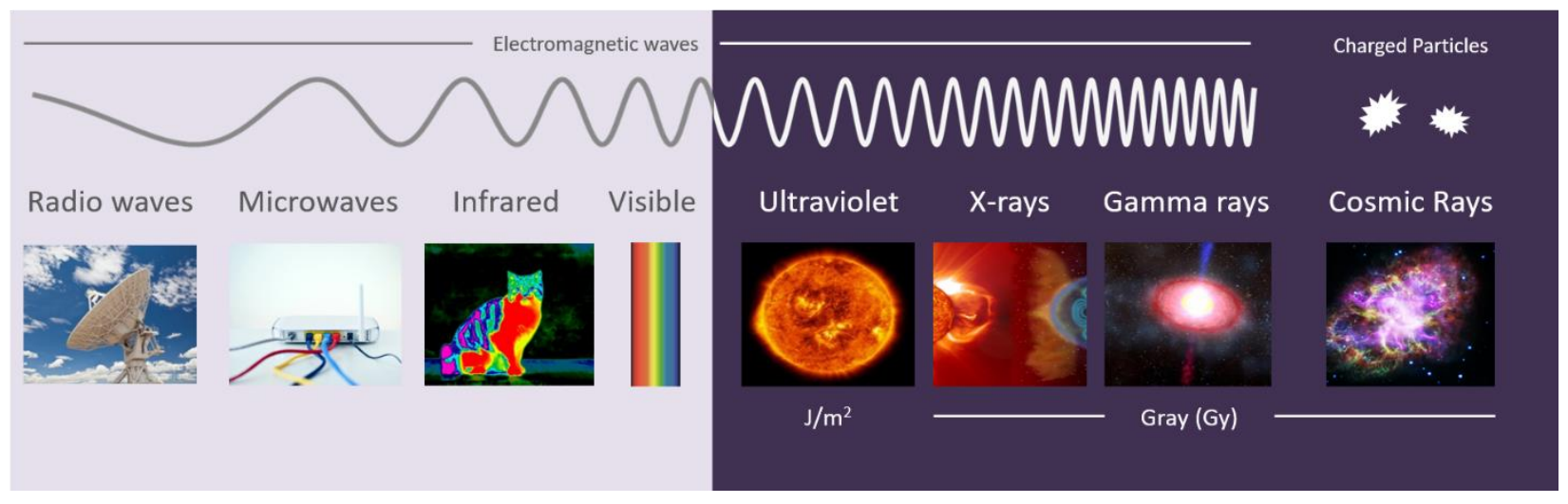

Figure 1. Different types of radiation. Electromagnetic waves include infrared radiation, used in our microwaves and Wi-fi routers, and visible light (the colors of the rainbow) as well as UV, X-rays and Gamma rays. Space radiation can be defined as including UV, $\mathrm{X}$-rays, Gamma rays as well as cosmic rays (i.e. charged particles). Non-ionizing UV radiation is measured in Joules per square meter $\left(\mathrm{J} / \mathrm{m}^{2}\right)$ whereas ionizing radiation (e.g. X-rays, Gamma rays and cosmic rays) are measured in Gray (Gy). 
There are only few facilities in the world able to accelerate heavy ions, such as the Helmholtz Centre for Heavy lon Research (GSI) in Darmstadt, Germany, or the Heavy lon Medical Accelerator (HIMAC) Chiba, Japan (GSI, 2021; NIRS-HIMAC, 2021). Depending on the ion species, the linear energy transfer (LET, $\mathrm{keV} / \mu \mathrm{m})$ rate differs significantly. Different LETs deposit different amounts of energy in the biological sample, and thus cause different cellular damage (Moeller et al., 2010a). However, while exposure to individual types of radiation is relatively easy, exposure to multiple types of radiation simultaneously is very challenging.

\subsubsection{Mars surface conditions}

Each planetary body, e.g. the Moon or Mars, has their own set of extreme environmental conditions, e.g. atmosphere (or lack thereof), gravity, and radiation exposure that will impact all living systems on it. Space agencies and private companies have joined efforts in human space exploration where Mars is one of the primary goals (SpaceX, 2021; Witze, 2020). However, with Mars being far from Earth (more specifically $6.79 \times 10^{7} \mathrm{~km}$ away), and with crewed flight taking 6-8 months in one direction, one can say that traveling to the red planet is a challenging endeavor (Cucinotta et al., 2010). Once in the martian surface, the challenge continues. Undeniably, the martian surface is an extreme environment. It is highly desiccating, it has low atmospheric pressure (5-10 mbar) and extreme temperatures $\left(-73^{\circ} \mathrm{C}\right.$ to $20{ }^{\circ} \mathrm{C}$ ) (Barlow, 2008). The Mars atmosphere is composed mostly of carbon dioxide $\left(96 \% \mathrm{CO}_{2}, 1.9 \% \mathrm{Ar}, 1.9 \% \mathrm{~N}_{2}, 0.14 \% \mathrm{O}_{2}\right.$, $0.07 \%$ CO) (Mahaffy et al., 2013). Moreover, it's thin atmosphere and weak magnetosphere (Acuña et al., 2001) let through more radiation compared to what happens on Earth. Solar non-ionizing UV radiation $(100-400 \mathrm{~nm}$ ) fluence on the Mars equator is $\sim 5 \mathrm{~mW} / \mathrm{cm}$ (Schuerger et al., 2003), and ionizing radiation can reach $225 \mu \mathrm{Gy} /$ day (Hassler et al., 2014).

Regrettably, microbial exposure to the real Mars environment is not yet possible, and accurate and simultaneous multi-factorial Mars-like environmental simulations are hard to achieve. Nonetheless, some Mars simulation platforms allow for simultaneous exposure to multiple conditions. For instance, the Mars Environmental Simulation Chamber (MESCH) (Jensen et al., 2008), or the Space Environmental Chamber for Planetary Studies (Santos and de Castro, 2021; Ramachandran et al., 2020). There is also the Mars Simulation Facility (MSF) in DLR Berlin which exposed the fungus Cryomyces antarcticus (Zakharova et al., 2014). What's more, the Mars simulation chamber (MSC) has been used to expose different microorganisms, using elevated radiation and desiccation paired with low pressure and temperature alongside a Mars gas mixture (Cortesão et al., 2019; Jensen et al., 2008; Motamedi et al., 2015; Schuerger et al., 2008). The MARTE vacuum chamber of the Center for Astrobiology (CAB), in Madrid, was reported to be able to simulate martian hydrological cycles (Sobrado et al., 2014; Sobrado, 2020). Besides, scientific balloons can also be used to expose microbes to simulated martian environment. These balloons can fly up to the Earth's stratosphere, where temperature fluctuations and UV fluences are similar to those on Mars (Smith and Sowa, 2017). Moreover, combined Mars simulation experiments with real space radiation (i.e. outside the space station) have also been performed. For instance, the Biology and Mars Experiment (BIOMEX) experiments aboard the ISS combined real outer space radiation exposure with simulated Mars atmosphere and/or regolith, were a successful platform in space microbiology research (Aerts et al., 2020; Onofri et al., 2018). Moreover, the characterization of fungal isolates from terrestrial Mars-analog sites is also used to inform on how terrestrial microbes could survive in the martian environment. For instance, 
characterization of microbes isolated from Mars analog habitats such as Antarctica (Van Ombergen et al., 2021), or the Atacama Desert (Santiago et al., 2018).

\subsection{Filamentous fungi on Earth and in Space}

Filamentous fungi are ubiquitous in the environment and can present themselves both as opportunities and as threats both on Earth and space habitats alike. On the one hand, they are indoor colonizers, foodcontaminators and opportunistic human pathogens (Brown et al., 2012). As an example, Aspergillus fumigatus causes $90 \%$ of worldwide fungal infections, followed by Aspergillus flavus and $A$. niger (Bongomin et al., 2017), and the Leading International Fungal Education (LIFE) portal estimated the burden of serious fungal infections as over 5.7 billion people, i.e. over $80 \%$ of the world's population (Denning, 2017). On the other hand, filamentous fungi such $A$. niger play a major role in modern-day biotechnology, producing a wide-range of valuable compounds (e.g. food, enzymes, antibiotics, etc.), and will likely play an essential role in establishing a circular bioeconomy, both on Earth and in Space (Cortesão et al., 2020; Meyer et al., 2011, 2020).

The modern built environment creates unique habitats that are ideal for the survival and interaction of microbial communities, with no exception for fungi. Fungal contaminations are commonly found in airplane fuel tanks, hospital air-filtration systems and surgical instruments (Caggiano et al., 2014; McNamara et al., 2005), as well as on the International Space Station. In fact, fungi have been found on several areas of the ISS: windows, walls, water recycling and air-filtration systems (Checinska et al., 2015; Sielaff et al., 2019; Novikova et al., 2006)(Figure 2). There is a notorious picture of mold fungi growing on the wall of the ISS, where astronauts would hang their sport-clothes to dry (Figure 2). The sweat, i.e. water full of nutrients, would condensate on the wall and, coupled with controlled humidity and temperature created the perfect environment for fungal growth. Analysis of ISS bioaerosols have detected fungal spores (Haines et al., 2019), and fungi have also been found as part of the astronaut microbiome (Satoh et al., 2021). Filamentous fungi have been found colonizing diverse surfaces aboard the ISS and in spacecraftassembly clean rooms on Earth (Novikova et al., 2006; Regberg et al., 2020; Satoh et al., 2016). Surface colonization is particularly challenging, since it can lead to material biodegradation. In fact, the fungus $P$. rubens was found responsible for degradation of surfaces in the Mir station (Klintworth et al., 1999). A detailed review on fungi in space stations Mir and ISS, with a special focus on the risk of mycotoxin exposure in space habitats is provided by (De Middeleer et al., 2019). 

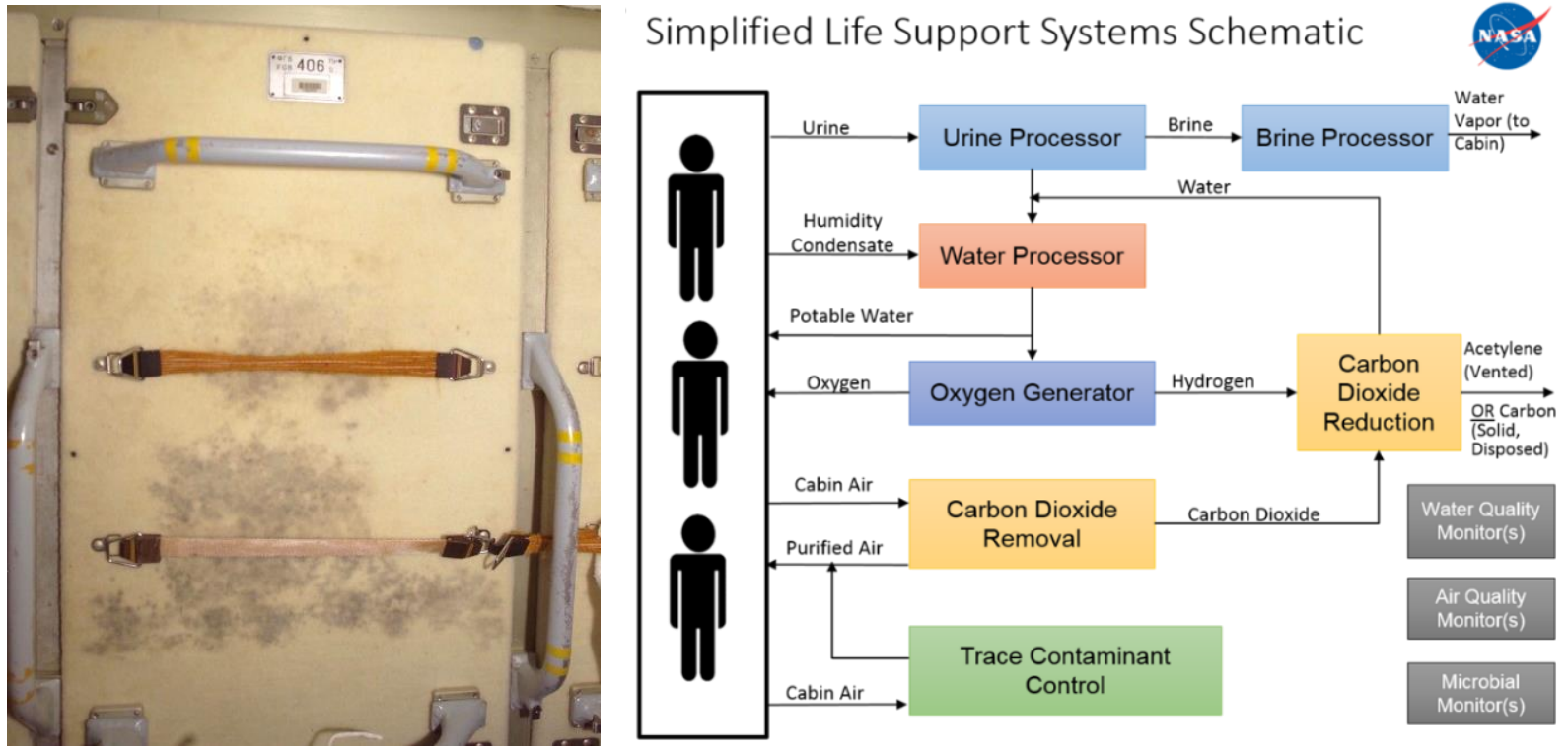

Figure 2. On the left, fungal growth on a panel wall of the ISS. On the right, a simplified scheme of connected life support systems of the ISS living environment. Credit: NASA

\subsubsection{Aspergillus niger as a model fungus for space microbiology}

Aspergillus niger is an industrially and medically relevant filamentous fungus (Ascomycete). The industrial wild-type industrial $A$. niger strain N402 (ATCC 64974), has a genome with $35.57 \mathrm{Mb}, 49.6 \%$ GC content, 8 chromosomes and more than 11,000 genes (Pel et al., 2007). Searching for $A$. niger related literature in PubMed reveals more than 10.000 entries (PubMed-NCBI, 2021). A. niger has highly pigmented and resistant spores which are airborne and can easily colonize different habitats, foods and animals. It is also a human opportunistic pathogen, and inhalation of its spores can cause invasive pulmonary aspergillosis and ear infections in immunocompromised humans (Person et al., 2010; Schuster et al., 2002). A. niger has been found as a common isolate of the Mir space station and ISS microbiota, and was also found in intensely sterilized spacecraft assembly facilities (Be et al., 2017; Checinska et al., 2015; Makimura et al., 2001; Venkateswaran et al., 2014). A. niger spores are of particular interest for space microbiology since they can potentially endure space travel and impact planetary protection policies. Its surface-associated colony growth and biofilm formation is also of interest to space habitats, since they impact both spacecraft surface integrity and life-support systems (Ramage et al., 2011). Moreover, $A$. niger can be cultivated over a wide range of conditions: temperature $\left(10-50^{\circ} \mathrm{C}\right), \mathrm{pH}(2-11)$, salinity $(0-34 \%)$ and water activity $(0.6$ - $1 \mathrm{aw}$ ), as well as under nutrient-poor or nutrient-rich conditions (Meyer et al., 2011). When grown in solid-media static and aerial conditions, $A$. niger colonies are considered analogs to lung-infection biofilms and to surface-associated contaminations (Beauvais et al., 2014). When grown in liquid-media submerged conditions, A. niger forms dispersed or pelleted macromorphological units that can be used as production and secretion system of valuable biotechnological compounds (Cairns et al., 2019; Meyer et al., 2011). With a well-annotated genome sequenced in 2007 (Pel et al., 2007) and well established advanced genetic engineering and system biology tools, such as a high-quality transcriptomic database (Schäpe et al., 2019), A. niger is as an established model system for biotechnology, and microbiology (Cairns et al., 2018; Geib 
and Brock, 2017; Schuetze and Meyer, 2017). Currently, A. niger is used in industrial scale citric acid biosynthesis with submerged fermentation techniques (Behera, 2020; Currie, 1917; Tong et al., 2019). Citric acid is extensively used in different areas of our daily life, from food industry (e.g. as acidulate, $\mathrm{pH}$ adjuster, flavoring agent, antioxidant additive, or ice-cream emulsifier) to cosmetic and industrial applications (e.g. as a metal ion chelating agent) (He et al., 2018; Lee and Arepally, 2012; Soccol et al., 2006). Indeed, as a key-player in modern-day biotechnology on Earth, $A$. niger will likely play an important role in fungal-based biotechnology in space. Astronauts settled in space habitats (e.g. Moon or Mars) will require resource-independence from Earth, and filamentous fungi, such as $A$. niger can be valuable companions in the production of needed compounds such as pharmaceuticals (Cortesão et al., 2020). As a Eukaryote, A. niger's molecular machinery is closely related to that of plants and humans (i.e., in comparison to bacteria, or archaea). This facilitates not only the utilization of $A$. niger has a valuable eukaryotic expression system, but also facilitates the transfer of knowledge concerning molecular mechanisms behind the adaptation and resistance to extreme terrestrial and extraterrestrial environments to eukaryotic systems.

\subsubsection{Fungal stress resistance mechanisms}

Understanding how fungi adapt and resist to terrestrial extremes will help us understand how they could resist and adapt to environmental conditions beyond Earth, in particular to the most challenging ones such as radiation. However, compared with other microbes (e.g. Escherichia coli or Bacillus subtilis) or with humans, the filamentous fungi stress response is not yet deeply characterized. Overall, when exposed to stress conditions cells have two main lines of defense: damage prevention and damage repair. When these lines of defense fail, and damage is too great, cells can enter cell-cycle arrest (apoptosis) (Goldman et al., 2002). Damage prevention is mostly dependent on stress response, with a particular focus on the cell wall. Damage repair is mostly dependent on the DNA damage response (DDR), with a focus on DNA repair.

Stress response in fungi is complex and depends on pathway cross-communication. In sum, stress sensors activate the Mitogen-activated protein kinase (MAPK) cascades, i.e. signalling pathways that include the Cell Wall Integrity (CWI) pathway, the High Osmolality-Glycerol (HOG) pathway and the Pheromone module pathway (Hagiwara et al., 2016) (Figure 3). These MAPK pathways are believed to crosscommunicate with other pathways such as oxidative stress pathway, the calcium-responsive pathway and likely also DNA damage response (or DNA repair) (de Castro et al., 2019; Fuchs and Mylonakis, 2009). MAPK allows fungal adaptation to a wide range of stress conditions, from osmotic stress, to cell wall stress, development, secondary metabolite production, and spore stress resistance (Hagiwara et al., 2016). Interestingly, a relation between oxidative stress and secondary metabolism has been suggested in filamentous fungi (Montibus et al., 2015), and possibly also between osmotic stress and secondary metabolism (Duran et al., 2010) (Figure 3). The pheromone module pathway is implicated in the regulation of fungal development, secondary metabolism and pathogenicity (Frawley and Bayram, 2020). 
Stress response in the genus Aspergillus

\begin{tabular}{|c|c|c|c|}
\hline \multicolumn{3}{|c|}{ MAPK cascade } & \multirow[b]{2}{*}{ Oxidative stress } \\
\hline $\begin{array}{l}\text { Pheromone } \\
\text { module } \\
\text { pathway }\end{array}$ & $\begin{array}{c}\text { Cell Wall } \\
\text { Integrity (CWI) } \\
\text { pathway }\end{array}$ & $\begin{array}{c}\text { High Osmolality } \\
\text { Glycerol (HOG) } \\
\text { pathway }\end{array}$ & \\
\hline $\begin{array}{l}\text { Hyphal growth } \\
\text { (actin organization } \\
\text { Rho-GTPases) }\end{array}$ & $\begin{array}{c}\text { Cell wall } \\
\text { biosynthesis }\end{array}$ & Osmotic stress & DNA repair \\
\hline $\begin{array}{c}\text { Asexual } \\
\text { development } \\
\text { (sporulation) }\end{array}$ & Virulence & $\begin{array}{c}\text { Spore stress } \\
\downarrow\end{array}$ & $\begin{array}{c}\text { Calcium-responsive } \\
\text { pathway }\end{array}$ \\
\hline $\begin{array}{l}\text { Spore viability } \\
\text { Secondary } \\
\text { metabolite } \\
\text { production }\end{array}$ & $\begin{array}{l}\text { Secondary } \\
\text { metabolite } \\
\text { (pigments) }\end{array}$ & $\begin{array}{l}\text { Compatible solutes } \\
\text { (trehalose, mannitol) } \\
\text { Conidia-specific } \\
\text { catalase (CatA) }\end{array}$ & Cell-cycle arrest \\
\hline
\end{tabular}

Figure 3. Stress response in the genus Aspergilus. Multiple signaling pathways contribute to an efficient stress response. There are three main pathways via the MAPK cascade: the Pheromone module pathway; the Cell Wall Integrity pathway, and the High Osmolality Glycerol pathway. These cross-communicate with other pathways, such as oxidative stress, DNA repair, calcium-responsive and cell-cycle arrest (Valiante et al., 2015, Fuchs and Mylonakis 2009; Braga et al., 2015; Hagiwara et al., 2016; de Castro et al., 2019; Frawley and Bayram 2020; Zhang et al., 2020).

Since damage typically acts first on the cell wall, one of the most important pathways in stress response is the CWI signaling pathway (Valiante et al., 2015). The fungal cell wall is essential for growth and for resisting environmental stresses, such that cell wall is a common target for antifungal drugs. Overall, $A$. niger's cell wall is rich in chitin $10-15 \%$ and $\alpha$-1,3-glucan (10-35\%)(Figure4). Fibrillar polysaccharides such as chitin and $\beta-1,3$-glucan are essential in the cell wall construction and good antifungal targets. Amorphous polysaccharides like $\alpha$-1,3-glucans, although not essential, have adhesive properties and stabilize the cell wall (Beauvais et al., 2014; Latgé, 2017). Remarkably, the fungal cell wall should be seen as flexible rather than a fixed component. It undergoes many changes from resting spore, to swollen spore, to germling, to hyphae (Figure 5). Indeed, the spore cell wall is different from the hyphal cell wall (Figure 4), the main difference being the outer layer (Garcia-Rubio et al., 2020) (Figure 4). The outer layer of the hyphal cell wall contains galactosaminogalactan - an important virulence factor (Beauvais et al., 2014); whereas the outer layer of spores is composed of hydrophobin rodlets and pigments (e.g. melanin) that confer hydrophobic properties and prevent immune recognition - spores being the infective morphotype. Indeed, both RodA (hydrophobin) and melanin are known key-players in fungal virulence. Melanin, in particular, is associated with reduced susception to antifungal drugs, and is known to have a strong antiphagocitic function (Cordero and Casadevall, 2017; Latgé, 2017).

Different fungal morphotypes (spore, germling and hyphae) have different cellular characteristics and thus different stress responses (Figure 5). Fungal spores are highly resistant structures able to withstand a widerange of extreme conditions. They have been known to resist harsh conditions, such as heat, low water 
activity as well as house-cleaning products, food-sterilization, and antifungal treatments (Esbelin et al., 2013; Segers et al., 2018; Singaravelan et al., 2008; Tribst et al., 2009). The high resistance of fungal spores is due to two main properties: the cell wall structure and its inner molecules.

Firstly, the spore's structure in itself - a multi-layered cell wall, with rodlet, pigment, cell wall, cell membrane (Figure 4), that helps to physically prevent the damage from reaching the most important molecule - the DNA. Moreover, CWI pathway plays a key role in remodeling and dealing with damage to the cell wall, especially upon revival after stress exposure (i.e. germination) (Figure 5). Response to cell wall stress in $A$. niger activates transcription of cell wall reinforcing genes and requires at least three known transcription factors (RmIA, MsnA and CrzA) (Damveld et al., 2005). Moreover, in A. niger cell wall stress normally responds with an increased production of chitin through RImA transcription factor that regulates the expression of genes agsA and gfaA, responsible for chitin synthesis (Fiedler et al., 2014; Latgé, 2017). Particularly important with regards to resisting space radiation are the pigments in the spore cell wall. Melanins are composed of indole- and phenol-type compounds that result in an insoluble and acid resistant material. They are able to adsorb all UV, visible and infrared radiation and are have also been reported to adsorb X-rays and Gamma rays (Casadevall et al., 2017). Indeed, melanotic fungi are commonly found in extreme environments such as Antarctic or Chernobyl. Some fungi are even able to harvest electromagnetic radiation and transduce this radiation into forms of energy (i.e. radiosynthesis or radiation-induced growth), suggesting the ability of melanotic fungi to be autotrophs (Casadevall et al., 2017). In filamentous fungi there are two different pathways for melanin production: Polyketide 1,8dihydroxynaphthalene (DHN) and 3,4-dihydroxyphenylalanine (L-DOPA) (Eisenman and Casadevall, 2012). In A. niger the DHN-melanin pigment has not been identified so far (Pombeiro-Sponchiado, 2017), and only three DHN-melanin gene orthologs (namely fwnA, olvA and brnA) seem to be present, in comparison to other DHN-melanin producing fungi (such as $P$. rubens and A. fumigatus) (Guzmán-Chávez et al., 2018; Jørgensen et al., 2011; Perez-Cuesta et al., 2020). A. niger strains with a deletion of fwnA, and thus defective in a polyketide synthase (PKS) present light-brown pigmentation, while disruptions of the ortholog gene alb1 in A. fumigatus have led to a white phenotype (Jørgensen et al., 2011; Perez-Cuesta et al., 2020). White $A$. niger strains have only been found after disruption of the pptA gene, regulating PKS and Non-Ribosomal-Pepetide Synthase (NRPS) enzymes. This suggests a more complex pigment synthesis in $A$. niger, with the likely synthesis of another pigment of either of polyketide or non-ribosomal peptide nature, that is responsible for the residual fawn color of $\Delta f w n A$ strains (Jørgensen et al., 2011).

Secondly, the spore counts with valuable inner molecules, such as antioxidants and compatible solutes that help to adapt to stress conditions (Figure 5). Accumulation of trehalose and mannitol - compatible solutes that act as protective molecules by creating high cytoplasmic viscosity - is facilitated by the spore's dormancy and minimal metabolic activity (Dijksterhuis et al., 2007; Thammahong et al., 2017). Compatible solutes were involved in heat resistance of Penicillium roqueforti (Punt et al., 2020) and in extreme water deprivation in Aspergillus sydowii (Jiménez-Gómez et al., 2020). Moreover, studies with Aspergillus wentii have identified compatible solutes as key-molecules to extend the biotic window, i.e. to extend the environmental limits where life is possible (Alves et al., 2015). In addition, a fungal spore-specific catalase (CatA) has been identified in $A$. fumigatus as an important molecule in oxidative stress response as a scavenger of hydrogen peroxide $\left(\mathrm{H}_{2} \mathrm{O}_{2}\right)$ (Paris et al., 2003). 
Hyphae Cell Wall

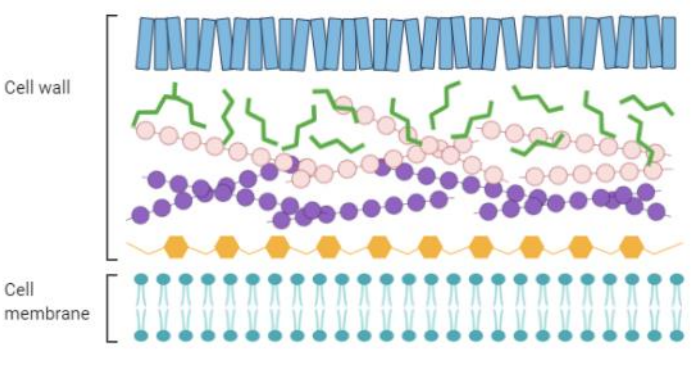

Spore Cell Wall

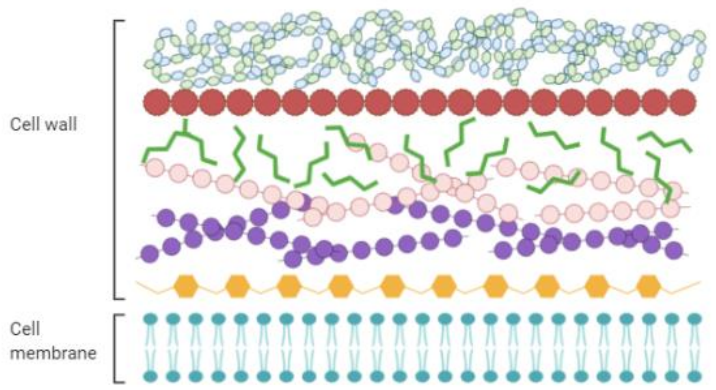

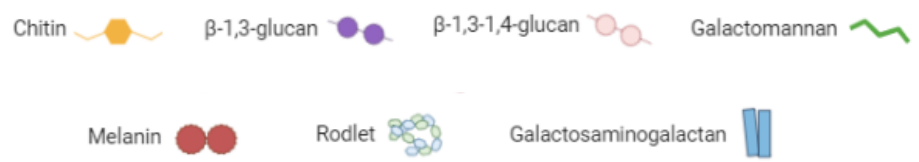

Figure 4. Differences between A. niger hyphae and spore cell wall. The outer layer of the hyphal cell wall contains galactosaminogalactan - an important virulence factor in A. fumigatus whereas the outer layer of conidia is composed of hydrophobin rodlets and pigments (e.g. melanin). Pigments are also important virulence factors and key-players in spore resistance (Beauvais et al., 2014; Latgé, 2017).

Radiation is the most limiting factor for space exploration, and, like other stressors, it acts first on cell wall. However, if damage cannot be prevented it will reach the DNA and threaten genomic integrity. Thus, an efficient DNA damage response that activates several DNA repair mechanisms and ensures genomic integrity is crucial for radiation-resistance. Radiation damage can be direct, e.g. DNA lesions, and lesions to the proteins, and lipids; or indirect, e.g. generated by reactive oxygen species (ROS), which can cause oxidative stress within cells (Hayyan et al., 2016; Horneck et al., 2006). Compared with prokaryotes and humans, DNA damage response in filamentous fungi is not well understood. Nevertheless, studies with Neurospora crassa (Inoue, 1999, 2011), Aspergillus fumigatus (Hartmann et al., 2011), Aspergillus nidulans (Goldman and Kafer, 2004) have been unravelling the mechanisms behind genome integrity and DNA repair. Different qualities of radiation affect the cell and the DNA in different ways, and therefore activate different DNA repair mechanisms. For instance, UV radiation induces mainly DNA photolesions such as cyclobutane pyrimidine dimers (CPDs) and 6-4 pyrimidine-pyrrolidine (Goldman et al., 2002). Indeed, photoreactivation via a DNA photolyase has been found to be one of the main pathways for DNA repair in filamentous fungi (Goldman et al., 2002). Nucleotide excision repair (NER) is also known to be the mostly used repair mechanism to deal with UV-induced single-strand breaks (or SSBs) (Goldman et al., 2002).

In contrast, ionizing radiation mainly causes double-strand breaks (or DSBs). Here, there are two main repair pathways for dealing with double-strand breaks: Non-Homologous End Joining (NHEJ) and Homologous Recombination (HR). NHEJ is highly efficient, although error-prone, in filamentous fungi such as $A$. niger. In fact, in the production of new strains it was found that removing NHEJ-repair capabilities results in a higher efficiency of gene targeting through homologous integration (Meyer et al., 2007). Interestingly, one key difference between bacterial and fungal DNA damage response was found to be based on the fact that multinucleated hyphae and multiple genome copies can facilitate the repair of DNA lesions (Goldman et al., 2002). Here, DNA damage checkpoints can enable cell-cycle arrest, and advice the 
cell to enter facilitated cell death (or apoptosis). What's more, a difference between radiation resistance of aseptate and septate hyphae has also been identified, where septate hyphae can compartmentalize damage and better ensure genomic integrity (Goldman et al., 2002). Recently, studies with $A$. fumigatus propose that the protein DefA - required for proper fungal growth and development - plays a crucial role in protecting hyphae against DNA damage stresses. The study suggests that the velvet protein VeA - a transcriptional regulator involved in sporulation that has a DNA-binding motif - controls levels of DefA and therefore proposes a VeA-DefA mediation of DNA damage response in A. fumigatus spores (Shin et al., 2016). How soon after exposure to stress conditions the different types of damage are repaired, i.e. whether upon spore revival (germination) or if only in hyphal growth, is not yet known (Baltussen et al., 2020). Studies with A. fumigatus have identified the fungal-specific histone acetyltransferase Rtt109 as being involved in DNA damage response; however, the connection of Rtt109 with spore germination was discarded (Zhang et al., 2020).

Overall, the ability of filamentous fungi such as A. niger to withstand and adapt to various stress conditions is a complex process, and interconnects many physiological and molecular properties. Although there is still much to learn when it comes to stress response and DNA repair in Aspergilli, fungal pathogenicity and stress resistance are undoubtfully interconnected (Abad et al., 2010; Brown and Goldman, 2016; Hartmann et al., 2011).

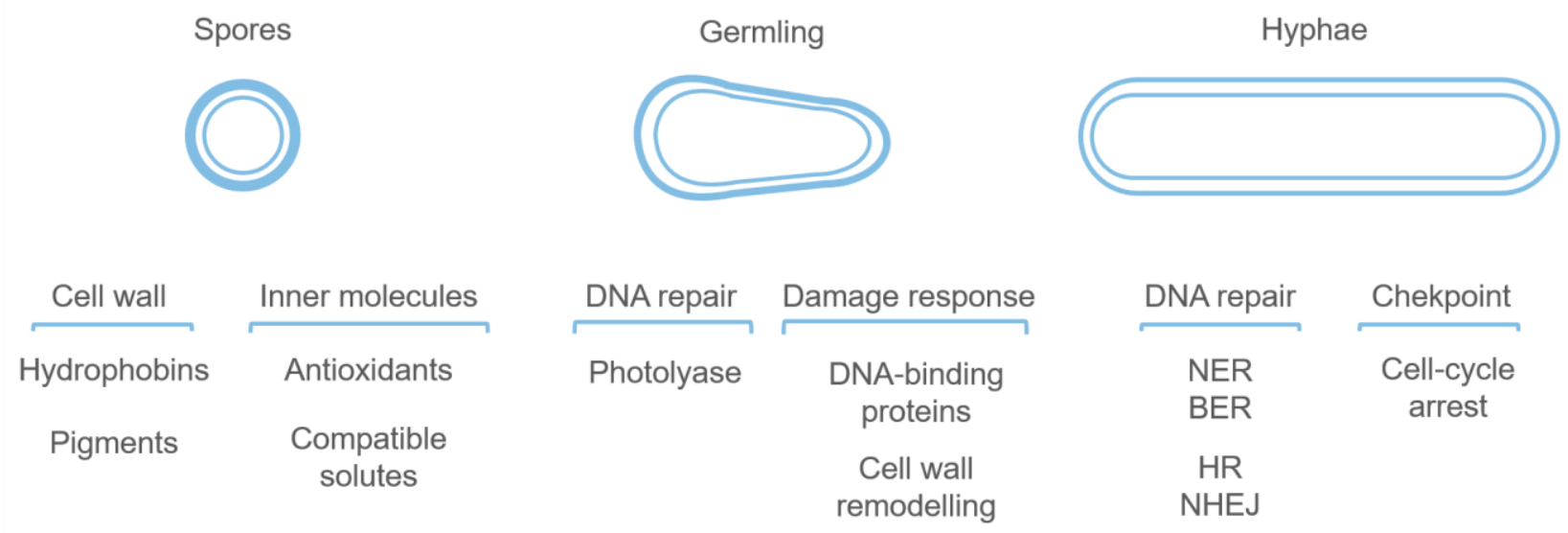

Figure 5. Aspergillus morphotypes and their main stress response properties. Spores resistance is highly dependent on the multilayered cell wall, in particular the outer layer (with hydrophobins and pigments), as well as and inner molecules (such as antioxidants and compatible solutes); Upon spore revival, the germling stress reponse involves efficient DNA repair, likely through an initial approach via DNA photolyase, as well as a coordinated DNA damage response through action of DNA-binding proteins and efficient cell wall remodeling. Later on, hyphae count with efficient DNA repair mechanisms (BER, NER, HR and NHEJ) as well as DNA damage checkpoints that can advise the cell to arrest its cycle and possibly entering apoptosis (Baltussen et al., 2020; Shin et al., 2016; Zhang et al., 2020). 


\subsubsection{Real and simulated space experiments with filamentous fungi}

Currently, the best way to expose microorganisms to the space environment is to have space experiments aboard the ISS. These space experiments can be set either on the outside of the space station (i.e. testing the outer space environment with high doses of radiation, vacuum, and extreme temperatures), or on the inside the space station, i.e. testing the spaceflight microgravity, low dose radiation and controlled temperatures. Alternatively, space experiments can also take advantage of outer space to test microorganism survival to Lunar or Mars-simulated conditions. However, given space experiments have many limitations, many ground-based simulations platforms have been used to test microbial adaptation to space conditions (Rabbow et al., 2016). Table 1 presents a summary of real and simulated space experiments with filamentous fungi.

So far, most studies conducted on microbial viability to space radiation have been performed with bacterial spores (Moeller et al., 2014; Nicholson et al., 2012; Wassmann et al., 2010; Zammuto et al., 2018). In fact, the effect of different types of radiation in B. subtilis spores was tested already in Apollo 16, Spacelab 1, and D2 missions (Bucker and Horneck, 1975; Facius et al., 1979; Horneck et al., 1974, 1994). However, while knowledge of bacterial spore resistance mechanisms is extensive (Setlow, 2014), knowledge on fungal spore survival to extreme conditions is rather scarce. Early studies tested $A$. niger spore survival to ultra-high vacuum and solar radiation and hinted the high resistance of fungal spores where dried spore monolayers registered $24 \%$ survival after exposure to $\sim 16 \mathrm{~kJ} \mathrm{~m}^{-2}$ of UVB (280 - 320 $\mathrm{nm}$ )(Silverman et al., 1967). A later study tested Penicillium sp. and Aspergillus sp. spore resistance to gamma and e-beam radiation and revealed survival to high doses of radiation (Blank and Corrigan, 1995). Moreover a study that exposed Penicillium expansum to the outside of the spacecraft for 7 months, revealed morphological changes such as the increase of polysaccharide capsule and melanin layer in $P$. expansum (Dadachova and Casadevall, 2008). Recently, ESA space microbiology missions EXPOSE, EXPOSE$\mathrm{R}$ and EXPOSE-R2 exposed several microorganisms, including fungi, to the outside of the ISS in low Earth orbit, testing microbial survival to different outer space and Mars-simulated conditions (Rabbow et al., 2009, 2015, 2017).

To date, there are no studies on the best microgravity simulation method for filamentous fungi, however, several experiments with filamentous fungi have tested different methods. Cortesão et al., 2020 provide an historical review on the development of fungal experiments in the space environment, and the platforms used to expose the test organisms to both simulated and real microgravity. Overall, liquid cultures of $A$. niger and $P$. rubens (formerly P. chrysogenum) in HARV, at a rotation speed of 25 r.p.m., found no significant changes in spore germination, mycelium growth or cell wall integrity (Sathishkumar et al., 2014). Further studies with HARV found changes in $P$. rubens transcriptome of cell wall $A B C$ transporter as well as increase in isopenicillin N Acyltransferase under low shear modeled microgravity (Sathishkumar et al., 2016). Further studies that tested the growth of Aspergillus carbonarius in solid, static and aerial conditions under simulated microgravity by slow-clinorotation of 20 r.p.m., revealed no changes in cell growth or colony appearance, but showed an increase in organic acids (e.g. oxalic acid and citric acid) and changes in metabolite transportation (Jiang et al., 2019). A study exposing A. niger to simulated microgravity using the RPM (also known as 3-D clinostat) revealed no differences in morphology, growth or susceptibility to antifungal agents (Yamazaki et al., 2012). Further investigations on morphology and antifungal susceptibility of ISS isolate strains of the fungi Penicillium sp., Aspergillus sp. and Cladosporium sp. found no significant differences to ground strains (Satoh et al., 2016). Additional studies with A. niger 
investigating changes in proteome showed that the ISS isolate had increased abundance of proteins involved in starvation response, oxidative stress, cell wall modulation and nutrient acquisition (Romsdahl et al., 2018). A follow-up study with the same strain, revealed enhanced production levels of therapeutically relevant secondary metabolites in the ISS isolate (Romsdahl et al., 2020). Another two studies with $A$. fumigatus revealed but found increased virulence, and increased abundance in proteins involved in stress responses, carbohydrate and secondary metabolism in ISS isolates (Knox et al., 2016; Blachowicz et al 2019a). In addition, A. nidulans colony growth on solid media (agar) aboard the ISS for 47 days, identified changes in secondary metabolite production (Romsdahl et al., 2019). Investigations with a Fusarium oxyporum ISS isolate compared with ground strains revealed potential changes in virulence (Urbaniak et al., 2019). Research on Ulocladium chartarum colony growth aboard the ISS, exposed to both spaceflight microgravity and to ionizing radiation doses of $150 \mu \mathrm{Gy}$, revealed reduced growth rate of aerial mycelium (Gomoiu et al., 2013). Exposure of the yeast Saccharomyces cerevisiae to real microgravity reported random budding patterns, reduced invasive filamentous growth and up-regulation of proteins related to anaerobic conditions (Van Mulders et al., 2011).

When investigating microbial adaptation to Mars surface conditions, there has been extensive studies on bacterial spores (Cortesão et al., 2019; Newcombe et al., 2005; Schuerger et al., 2003). Despite relevance of fungal spore resistance to space and Mars conditions due to planetary protection measures and potential for space biotechnology, few studies have addressed filamentous fungi resistance to Mars surface conditions. Previous studies investigated the survival of $A$. niger and $B$. subtilis spores in dark (non UV-irradiated) extreme conditions of the Atacama Desert up to 15 months (Mars analog), where A. niger spores were more resistant than $B$. subtilis spores (30\% versus $15 \%$ survival)(Dose et al., 2001). Another study using a stratospheric balloon as a Mars-analog investigated the survival of pigmented spores of the fungus Fuligo spectica, showed that spores were viable after $9 \mathrm{~h}$ (Díez et al., 2020). In addition, the Lichens and Fungi Experiment (LIFE) on EXPOSE-E mission and later the BIOMEX experiment on EXPOSE-R2 mission, have exposed the highly melanized fungus Cryomyces antarcticus aboard the ISS (de Vera et al., 2019). This fungus was isolated from cryptoendolithic microbial communities in the McMurdo Dry Valleys in Antarctica, and has been exposed to several astrobiology and space microbiology related experiments, from simulated Mars conditions aboard the International space station (de Vera et al., 2019; Onofri et al., 2008, 2012; Selbmann et al., 2018) to high doses of space radiation (Pacelli et al., 2017; Selbmann et al., 2018), as well as to accelerated helium ions combined within Mars regolith (Pacelli et al., 2020). Recently, a study investigated the proteome and metabolome of twelve fungal strains isolated from Chernobyl, and one A. fumigatus strain isolated from the ISS after exposure to Mars conditions and UV-C irradiation. Here, A. fumigatus was shown to survive for $30 \mathrm{~min}$ in the Mars-simulated environment, and to have $20 \%$ survival after exposure to $4000 \mathrm{~J} / \mathrm{m}^{2}$ (Blachowicz, et al., 2019b). Besides, fungal diversity has been studied in Mars analog sites such as the Atacama Desert (Santiago et al., 2018), and rock-associated fungi from Atacama and Antarctic are being tested for their bioactive compounds and potential human pathogenicity (Gonçalves et al., 2016, 2017). Interestingly, studies with Aspergillus penicillioides have shown its ability to tolerate extremely low water activity (aw $=0.585)$, which challenges the limits to life in extraterrestrial environments such as Mars (Stevenson et al., 2017). 
Table 1. Real and simulated space experiments with filamentous fungi

\begin{tabular}{|c|c|c|c|c|c|}
\hline Fungus & Space Condition & Exposure & $R / S$ & Effect of space condition & Reference \\
\hline \multicolumn{6}{|c|}{ Aspergillus niger } \\
\hline & Microgravity & $\begin{array}{l}\text { HARV, Liquid } \\
\text { culture }\end{array}$ & $S$ & No changes in stress response & $\begin{array}{l}\text { (Sathishkumar et } \\
\text { al., 2014) }\end{array}$ \\
\hline & $\begin{array}{l}\text { Microgravity, } \\
\text { Radiation }\end{array}$ & ISS isolate & $\mathrm{R}$ & $\begin{array}{l}\text { Enhanced production of naphto- } \\
\text { Y-pyrones and secondary } \\
\text { metabolites (pyranonigrin) }\end{array}$ & $\begin{array}{l}\text { (Romsdahl et al., } \\
\text { 2020) }\end{array}$ \\
\hline & Microgravity & & $\mathrm{S}$ & $\begin{array}{l}\text { No differences in morphology, } \\
\text { growth asexual development or } \\
\text { antifungal susceptibility }\end{array}$ & $\begin{array}{l}\text { (Yamazaki et al., } \\
\text { 2012) }\end{array}$ \\
\hline & $\begin{array}{l}\text { Cosmic rays } \\
\text { Gamma rays }\end{array}$ & $\begin{array}{l}\text { Spore } \\
\text { suspensions }\end{array}$ & $\mathrm{S}$ & $\begin{array}{l}\text { Survival } L_{90}=0.245 \mathrm{kGy}, \\
\text { Survival } \mathrm{LD}_{90}=0.199 \mathrm{kGy}\end{array}$ & $\begin{array}{l}\text { (Blank and Corrigan, } \\
\text { 1995) }\end{array}$ \\
\hline & UV-B radiation & Dried spores & $\mathrm{S}$ & $24 \%$ survival to $1.6 \mathrm{~kJ} / \mathrm{m} 2$ & $\begin{array}{l}\text { (Silverman et al., } \\
\text { 1967) }\end{array}$ \\
\hline & $\begin{array}{l}\text { Microgravity and } \\
\text { Radiation }\end{array}$ & ISS & $\mathrm{R}$ & No changes in spore viability & $\begin{array}{l}\text { (Gomoiu et al., } \\
\text { 2013) }\end{array}$ \\
\hline \multicolumn{6}{|c|}{ Aspergillus nidulans } \\
\hline & $\begin{array}{l}\text { Microgravity, } \\
\text { Radiation }\end{array}$ & ISS & $\mathrm{R}$ & $\begin{array}{l}\text { Changes in stress response and } \\
\text { secondary metabolites }\end{array}$ & $\begin{array}{l}\text { (Romsdahl et al., } \\
\text { 2019) }\end{array}$ \\
\hline & UV-B & $\begin{array}{l}\text { Spore } \\
\text { suspensions }\end{array}$ & $S$ & $\begin{array}{l}0.04 \text { CPDs per } 10 \mathrm{~kb} \text { at a dose of } \\
900 \mathrm{~J} / \mathrm{m}^{2}\end{array}$ & $\begin{array}{l}\text { (Nascimento et al., } \\
\text { 2010) }\end{array}$ \\
\hline \multicolumn{6}{|c|}{ Aspergillus fumigatus } \\
\hline & $\begin{array}{l}\text { Microgravity, } \\
\text { Radiation }\end{array}$ & ISS isolate & $\mathrm{R}$ & $\begin{array}{l}\text { Enhanced growth, increased } \\
\text { virulence. }\end{array}$ & (Knox et al., 2016) \\
\hline & $\begin{array}{l}\text { Microgravity, } \\
\text { Radiation }\end{array}$ & ISS isolate & $\mathrm{R}$ & $\begin{array}{l}\text { Increased abundance in proteins } \\
\text { involved in stress responses, } \\
\text { carbohydrate and secondary } \\
\text { metabolism }\end{array}$ & $\begin{array}{l}\text { (Blachowicz et al., } \\
\text { 2019a) }\end{array}$ \\
\hline & UV-B & $\begin{array}{l}\text { Spore } \\
\text { suspensions }\end{array}$ & $\mathrm{S}$ & $\begin{array}{l}1.62 \mathrm{CPDs} \text { per } 10 \mathrm{~kb} \text { at a dose of } \\
5400 \mathrm{~J} / \mathrm{m}^{2}\end{array}$ & $\begin{array}{l}\text { (Nascimento et al., } \\
2010 \text { ) }\end{array}$ \\
\hline & $\begin{array}{l}\text { Mars } \\
\text { UV-C }\end{array}$ & $\begin{array}{l}\text { ISS isolate, } \\
\text { Dried Spores, } \\
\text { MSC }\end{array}$ & $S$ & $\begin{array}{l}\text { Survive mars-like conditions for } \\
30 \mathrm{~min}, 20 \% \text { spore survival at } \\
4000 \mathrm{~J} / \mathrm{m}^{2}\end{array}$ & $\begin{array}{l}\text { (Blachowicz et al., } \\
\text { 2019b) }\end{array}$ \\
\hline \multicolumn{6}{|c|}{ Aspergillus carbonarius } \\
\hline & Microgravity & $\begin{array}{l}\text { Clinostat, } \\
\text { Solid media, } \\
20 \text { r.p.m. }\end{array}$ & $\mathrm{S}$ & $\begin{array}{l}\text { No effect in cell or colony } \\
\text { growth, but increased organic } \\
\text { acid production }\end{array}$ & (Jiang et al., 2019) \\
\hline \multicolumn{6}{|c|}{ Penicillium rubens (formerly P. chrysogenum) } \\
\hline & Microgravity & $\begin{array}{l}\text { HARV, Liquid } \\
\text { culture }\end{array}$ & $S$ & $\begin{array}{l}\text { Changes in cell wall; Increased } \\
\text { expression of Acyl-coenzyme: } \\
\text { isopenicillin N acyltransferase }\end{array}$ & $\begin{array}{l}\text { (Sathishkumar et } \\
\text { al., 2016) }\end{array}$ \\
\hline & Microgravity & $\begin{array}{l}\text { HARV, Liquid } \\
\text { culture }\end{array}$ & $S$ & $\begin{array}{l}\text { Number of mitochondria } \\
\text { increase }\end{array}$ & $\begin{array}{l}\text { (Sathishkumar et } \\
\text { al., 2014) }\end{array}$ \\
\hline & $\begin{array}{l}\text { Microgravity and } \\
\text { Radiation }\end{array}$ & ISS isolate & $\mathrm{R}$ & $\begin{array}{l}\text { No changes in morphology or } \\
\text { antifungal susceptibility }\end{array}$ & (Satoh et al., 2016) \\
\hline
\end{tabular}


Table 1.(Continuation)

\begin{tabular}{|c|c|c|c|c|c|}
\hline Fungus & Space Condition & Exposure & $R / S$ & Effect of space condition & Reference \\
\hline \multicolumn{6}{|c|}{ Penicillium expansum } \\
\hline & Microgravity & ISS, outside & $\mathrm{R}$ & Increase of polysaccharide capsule & (Dadachova and \\
\hline & and Radiation & & & and melanin layer & Casadevall, 2008) \\
\hline \multicolumn{6}{|c|}{ Fusariun oxysporum } \\
\hline & $\begin{array}{l}\text { Microgravity } \\
\text { and Radiation }\end{array}$ & ISS isolate & $\mathrm{R}$ & $\begin{array}{l}\text { Higher abundance of polyketide } \\
\text { synthase domains }\end{array}$ & $\begin{array}{l}\text { (Urbaniak et al., } \\
\text { 2019) }\end{array}$ \\
\hline \multicolumn{6}{|c|}{ Cryomyces antarcticus } \\
\hline & $\begin{array}{l}\text { Microgravity, } \\
\text { Space Radiation, } \\
\text { Mars }\end{array}$ & $\begin{array}{l}\text { EXPOSE-E, } \\
\text { EXPOSE-R2 }\end{array}$ & $\mathrm{R} / \mathrm{S}$ & $\begin{array}{l}12 \% \text { survival to full outer space, } \\
\text { including cold, ionizing and UV } \\
\text { radiation up to } 900 \mathrm{~kJ}\end{array}$ & $\begin{array}{l}\text { (de Vera et al., } \\
\text { 2019; Onofri et } \\
\text { al., 2012, 2018) }\end{array}$ \\
\hline & $\begin{array}{l}\text { X-rays, UV, } \\
\text { Cosmic Rays }\end{array}$ & $\begin{array}{l}\text { Commercial } \\
\text { radiation } \\
\text { sources }\end{array}$ & $\mathrm{S}$ & $\begin{array}{l}\text { Melanin protection at } 300 \text { Gy X-rays } \\
\text { and at } 1.5 \mathrm{kGy} \text { Deuterons (heavy } \\
\text { hydrogen) }\end{array}$ & $\begin{array}{l}\text { (Pacelli et al., } \\
\text { 2017, 2018, 2019; } \\
\text { Selbmann et al., } \\
2018 \text { ) }\end{array}$ \\
\hline & $\begin{array}{l}\text { Cosmic rays (He- } \\
\text { ions) with Mars } \\
\text { regolith }\end{array}$ & $\begin{array}{l}\text { Dried } \\
\text { colonies }\end{array}$ & $\mathrm{R}, \mathrm{S}$ & $\begin{array}{l}\text { Survival up to } 1 \mathrm{kGy} \text {, preservation of } \\
\text { DNA and melanin - still detectable } \\
\text { after exposure (biosignatures) }\end{array}$ & $\begin{array}{l}\text { (Pacelli et al., } \\
2019,2020)\end{array}$ \\
\hline \multicolumn{6}{|c|}{ Neurospora crassa } \\
\hline & $\begin{array}{l}\text { X-rays } \\
\text { Cosmic Rays }\end{array}$ & $\begin{array}{l}\text { Commercial } \\
\text { radiation } \\
\text { sources }\end{array}$ & $\mathrm{S}$ & $\begin{array}{l}\text { NHEJ-deficiency led to differences in } \\
\text { survival between X-ray and heavy } \\
\text { ions ( } \mathrm{Ar} \text { and Fe) }\end{array}$ & (Ma et al., 2018) \\
\hline
\end{tabular}

\begin{tabular}{|c|c|c|c|c|}
\hline $\begin{array}{l}\text { Microgravity } \\
\text { Radiation }\end{array}$ & $\begin{array}{l}\text { ISS, Solid } \\
\text { media }\end{array}$ & $\mathrm{R}$ & $\begin{array}{l}\text { Formation of microcolonies, changes } \\
\text { in colony growth, no changes in spore } \\
\text { viability }\end{array}$ & $\begin{array}{l}\text { (Gomoiu et al., } \\
2013,2016)\end{array}$ \\
\hline \multicolumn{5}{|l|}{ Saccharomyces cerevisiae } \\
\hline $\begin{array}{l}\text { Microgravity } \\
\text { Radiation }\end{array}$ & $\begin{array}{l}\text { Soyus and } \\
\text { ISS }\end{array}$ & $\mathrm{R}$ & $\begin{array}{l}\text { Up-regulation of proteins linked to } \\
\text { anaerobic conditions; random } \\
\text { budding patterns; reduced invasive } \\
\text { growth }\end{array}$ & $\begin{array}{l}\text { (Van Mulders et } \\
\text { al., 2011) }\end{array}$ \\
\hline \multicolumn{5}{|l|}{ Sordaria macrospora } \\
\hline Microgravity & $\begin{array}{l}\text { Clinostat, } 4 \\
\text { r.p.m. }\end{array}$ & $\mathrm{S}$ & Changes in crossover & $\begin{array}{l}\text { (Henkel and Hock, } \\
\text { 1991) }\end{array}$ \\
\hline Microgravity & $\begin{array}{l}\text { Space } \\
\text { Shuttle and } \\
\text { Mir }\end{array}$ & $\mathrm{R}$ & $\begin{array}{l}\text { No changes in crossing-over } \\
\text { frequencies under microgravity. } \\
\text { Increase gene recombination } \\
\text { frequencies under heavy ion radiation }\end{array}$ & $\begin{array}{l}\text { (Hahn and Hock, } \\
\text { 1999) }\end{array}$ \\
\hline \multicolumn{5}{|l|}{ Knufia chersonesos } \\
\hline Microgavity & HARV & $\mathrm{S}$ & $\begin{array}{l}\text { No changes in morphology, } \\
\text { upregulation of enzyme involved in } \\
\text { the synthesis of (DNH) melanin }\end{array}$ & (Tesei et al., 2021) \\
\hline
\end{tabular}

$\mathrm{R} / \mathrm{S}=$ Real (R) or Simulated (S); HARV = High Aspect Ration Vessel; ISS = International Space Staion; UV = Ultraviolet Radiation. $\mathrm{LD}_{90}=$ dose at which there is $90 \%$ spore inactivation CPDs = cyclobutane pyrimidine dimers. r.p. $\mathrm{m}=$ revolutions per minute; $\mathrm{MSC}=$ Mars Simulation Chamber. 


\subsection{Objective}

Despite the relevance to future space exploration endeavors, in particular in crewed long-term missions, the limits of fungal spore resistance to both spaceflight and Mars-like conditions are not yet known. Moreover, how fungal growth and biofilm formation can impact spacecraft safety and astronaut health is also not well understood. Thus, within the framework of this thesis, the industrial A. niger strain N402 ATCC 64974 was used as model organism to study the effects of space radiation and simulated martian environment in spore survival; and to investigate the effect of simulated microgravity in colony growth and biofilm formation. Additionally, in the scope of this doctoral thesis, the filamentous fungus $P$. rubens DSM 1075 (previously known as $P$. chrysogenum) was used as a model organism for the design and establishment of a space microbiology experiment aboard the International Space Station. This thesis is divided in four main chapters, each answers the following scientific questions:

1. Can A. niger spores tolerate different types of space radiation? How does spore pigmentation affect survival?

Relevant publication: Cortesão, M., de Haas, A., Unterbusch, R., Fujimori, A., Schuetze, T., Meyer, v., and Moeller, R. (2020) Aspergillus niger spores are highly resistant to space radiation. Frontiers in Microbiology. 11:560

2. Does the martian-like environment affect the viability of $A$. niger spores? How does self-shielding (spore multilayer) impact survival?

Relevant publication: Cortesão, M. and Siems, K., Koch, S., Beblo-Vranesevic, K., Rabbow, E., Berger, T., Lane, M., James, L., Johnson, P., Waters, S.M, Verma, S.D., Smith, D.J., and Moeller, R (2021). MARSBOx: Fungal and bacterial endurance from a balloon-flown analog mission in the stratosphere. Frontiers in Microbiology. 12:601713.

3. How does simulated microgravity affect colony ultrastructure and spore vitality of $A$. niger when grown in static, aerial conditions? Is FwnA-derived pigmentation involved in adaptation to microgravity?

Relevant publication: Cortesão, M., Holland, G. Laue, M., Schuetze, T., Moeller, R and Meyer, V. Growth and biofilm formation of Aspergillus niger under simulated microgravity, npj Microgravity (submitted: NPJMGRAV-00634)

4. Which experimental design can be established to test biofilm formation of $P$. rubens in a real spaceflight biofilm experiment aboard the ISS?

Relevant publications: Zea, L., Nisar, Z., Rubin, P., Cortesao, M., Luo, J., McBride, S., Moeller, R., Klaus, D., Mueller, D., Varanasi, K., Mücklich, F., Stodieck L. "Design of a spaceflight biofilm experiment". Acta Astronautica 148, 294-300, 2018.

Cortesao, M., Rubin, P., Luo, J., Hellweg, C.E., Stodieck L. Mücklich, F., Klaus, D., Moeller, R., and Zea, L. "Controlling spaceflight fungal biofilms: the search for antimicrobial surfaces". International Astronautical Congress (IAC) 2018 


\title{
2. Chapter I: Aspergillus niger spores are highly resistant to space radiation
}

\author{
Publication \\ Cortesão, M., de Haas, A., Unterbusch, R., Fujimori, A., Schuetze, T., Mever, V., and Moeller, R. (2020) \\ Aspergillus niger spores are highly resistant to space radiation. Frontiers in Microbiology. 11:560 doi: \\ 10.3389/fmicb.2020.00560
}

\section{Author contribution}

Planning: $80 \%$, Experiment: $50 \%$, Manuscript: $75 \%$

$\mathrm{MC}, \mathrm{AH}$, and RU performed the experiments, analyzed the data, and wrote the manuscript. VM, TS, RM, and $\mathrm{AF}$ contributed to the conception and design of the study, and manuscript preparation. 


\section{OPEN ACCESS}

Edited by: André Antunes,

Macau University of Science and Technology, China

Reviewed by:

Douglas Galante,

National Center for Research in Energy and Materials (CNPEM),

Marta Filipa Simōes, Macau University of Science and Technology, China

*Correspondence:

Marta Cortesão marta.cortesao@dlr.de

Specialty section: This article was submitted to Extreme Microbiology, a section of the journal Frontiers in Microbiology

Received: 21 January 2020 Accepted: 16 March 2020 Published: 03 April 2020

Citation:

Cortesão M, de Haas A, Unterbusch R, Fujimori A, Schütze T, Meyer $V$ and Moeller $R$ (2020) Aspergillus niger Spores Are Highly Resistant to Space Radiation. Front. Microbiol. 11:560. doi: $10.3389 / f m i c b .2020 .00560$

\section{Aspergillus niger Spores Are Highly Resistant to Space Radiation}

\author{
Marta Cortesão ${ }^{1 *}$, Aram de Haas ${ }^{1}$, Rebecca Unterbusch ${ }^{1}$, Akira Fujimori², \\ Tabea Schütze ${ }^{3}$, Vera Meyer ${ }^{3}$ and Ralf Moeller ${ }^{1}$
}

'Space Microbiology Research Group, Radiation Biology Department, Institute of Aerospace Medicine, German Aerospace Center, Cologne, Germany, ${ }^{2}$ Department of Basic Medical Sciences for Radiation Damages, National Institutes for Quantum and Radiological Science and Technology, Chiba, Japan, ${ }^{3}$ Chair of Applied and Molecular Microbiology, Institute of Biotechnology, Technische Universität Berlin, Berlin, Germany

The filamentous fungus Aspergillus niger is one of the main contaminants of the International Space Station (ISS). It forms highly pigmented, airborne spores that have thick cell walls and low metabolic activity, enabling them to withstand harsh conditions and colonize spacecraft surfaces. Whether $A$. niger spores are resistant to space radiation, and to what extent, is not yet known. In this study, spore suspensions of a wild-type and three mutant strains (with defects in pigmentation, DNA repair, and polar growth control) were exposed to X-rays, cosmic radiation (helium- and iron-ions) and UV-C (254 nm). To assess the level of resistance and survival limits of fungal spores in a long-term interplanetary mission scenario, we tested radiation doses up to $1000 \mathrm{~Gy}$ and $4000 \mathrm{~J} / \mathrm{m}^{2}$. For comparison, a 360-day round-trip to Mars yields a dose of $0.66 \pm 0.12$ Gy. Overall, wild-type spores of $A$. niger were able to withstand high doses of X-ray (LD90 = 360 Gy) and cosmic radiation (helium-ion $L_{90}=500$ Gy; and ironion $L_{90}=100$ Gy). Drying the spores before irradiation made them more susceptible toward X-ray radiation. Notably, $A$. niger spores are highly resistant to UV-C radiation $\left(\mathrm{LD}_{90}=1038 \mathrm{~J} / \mathrm{m}^{2}\right)$, which is significantly higher than that of other radiation-resistant microorganisms (e.g., Deinococcus radiodurans). In all strains, UV-C treated spores (1000 J/m²) were shown to have decreased biofilm formation (81\% reduction in wildtype spores). This study suggests that $A$. niger spores might not be easily inactivated by exposure to space radiation alone and that current planetary protection guidelines should be revisited, considering the high resistance of fungal spores.

Keywords: Aspergillus niger, A. niger spores, spore survival, space, radiation, X-ray, UV, international space station

\section{INTRODUCTION}

Radiation is the most challenging factor for life in the space environment (Horneck et al., 2010; Chancellor et al., 2014). On the one hand, the Sun emits UV radiation (non-ionizing), X-rays (ionizing electromagnetic waves) and solar flares (intense bursts of high-energy ionizing radiation) (Sliney, 2007). On the other hand, cosmic events such as supernova explosions or pulsars, emit 
galactic cosmic radiation (GCR) (Chancellor et al., 2018). GCR particle spectrum spans from light particles, such as hydrogenions ( $85 \%)$ and helium-ions ( $\mathrm{He}, 14 \%)$, to high charge $\mathrm{Z}$ and energy particles (HZE) like iron-ions (Fe, $0.03 \%$ ) (Horneck et al., 2010). Radiation shielding on the International Space Station (ISS) is provided by both the Earth's magnetosphere and the walls of the space station. However, not all types of radiation are easily shielded. For instance, HZE particles are still capable of penetrating current space vehicles (Chancellor et al., 2018). Protecting living systems from radiation becomes particularly challenging beyond low Earth orbit (LEO). Due to the absence of Earth's magnetosphere, space missions toward the Moon or Mars will be exposed to substantially higher radiation doses than those currently experienced on the ISS (Cucinotta et al., 2013; Chancellor et al., 2014, 2018; Narici et al., 2017). Studies on how radiation affects cells have identified two main types of damage: direct and indirect. Direct damage targets DNA (e.g., singleor double-strand breaks), proteins, or lipids. Whereas indirect damage is induced by the generation of reactive oxygen species (ROS) - which are produced by the interaction of radiation with cellular water molecules in a process called radiolysis (Cadet et al., 2015; Moeller et al., 2017).

Regardless of the damage, many microorganisms, especially spore formers, are able to withstand high radiation doses (Horneck et al., 2010; Moeller et al., 2010). Spores of the bacterium Bacillus subtilis are known to be highly resistant to extreme space conditions and therefore are currently being used as indicators for decontamination protocols and planetary protection policies (Kminek et al., 2019). However, while survival of bacterial spores has been extensively studied in both Earth and spaceflight contexts (Moeller et al., 2014; Setlow, 2014; Khodadad et al., 2017), survival of fungal spores has not. Samples from the ISS indoor microbiome identified Aspergillus niger as one of the most common fungal contaminants (Novikova et al., 2006; Checinska et al., 2015). Contrary to B. subtilis spores, which are formed as a response to stressful conditions, asexual spores of A. niger (i.e., conidia) are produced as a natural part of its life cycle (Krijgsheld et al., 2013). A. niger spores are highly pigmented and can be easily dispersed through the air which facilitates habitat colonization. Also, as an opportunistic human pathogen, inhalation of $A$. niger spores can lead to human respiratory infections (Silverman et al., 1967; Latgé, 1999; Esbelin et al., 2013). Thus, the ability of $A$. niger to survive and grow in the spaceflight environment is a potential threat to both astronaut health and spacecraft safety. Nonetheless, A. niger is also a well-established cell factory used in modernday biotechnology to produce various compounds such as proteins, enzymes, and pharmaceuticals (Meyer et al., 2015; Cairns et al., 2018). This makes A. niger a potential asset in long-term space missions, where astronauts will have to produce their own compounds of interest such as vitamins or antibiotics (Cortesão et al., 2020).

Despite the relevance of $A$. niger spores in the space context, it is not yet known whether they are able to withstand extreme space radiation conditions. Fungal spore survival generally depends on two main components. One is the spore cell wall, which helps to prevent radiation damage on the DNA.
The spore cell wall is composed of polysaccharides (mainly chitin and glucans), and is covered by an outer layer of rodlets (hydrophobins) and pigments (e.g., melanin). These outer layers of the cell wall make spores highly hydrophobic and highly pigmented (Beauvais et al., 2014). Pigments, such as melanin, are known to be involved in different cellular processes, from adhesion to virulence, as well as to protect cells from radiation-induced stress and ROS (Cockell and Knowland, 1999; Eisenman and Casadevall, 2012; Cordero and Casadevall, 2017). Melanized fungi have been reported in Chernobyl sites (Zhdanova et al., 2000; Casadevall et al., 2017), and some were even found displaying increased growth after X-ray irradiation (Dadachova et al., 2007). Previous studies have reported the presence of melanin in $A$. niger spores as an adaptive trait conferring resistance toward UV-A (315400) (Singaravelan et al., 2008). Moreover, studies on clinical isolates of Aspergillus fumigatus reported the involvement of DHN-melanin in UV-C protection. Here, loss of a polyketide synthase from the DHN-melanin pathway $(\Delta p k s P)$ resulted in decreased survival, when exposed to $100 \mathrm{~J} / \mathrm{m}^{2}$. This was not the case for an A. fumigatus strain isolated from the ISS, where loss of $p k s P$ did neither reduce nor increase viability (Blachowicz et al., 2020). However, a recent review emphasizes that pigment biosynthesis in Aspergillus species is not yet fully understood (Chang et al., 2020). Pigmentation in A. niger is particularly puzzling. The pigment spectrum of $A$. niger spores was shown to have two main absorbance peaks, which together absorb light in the entire VIS-spectrum and thus result in the black color. These are thought to be two distinct components - one green (peak at $\sim 575 \mathrm{~nm}$ ), and one brown component $(\sim 425 \mathrm{~nm})$ - and were both shown to be FwnA dependent (Jorgensen et al., 2011). FwnA is an ortholog of pksP, and deletion of the $f w n A$ gene $(\triangle f w n A)$ results in fawncolored (not white as for A. fumigatus) spores. Knowing if and how pigmentation is involved in spore resistance will be crucial to understand the limits of spore survival, which will, in turn, help develop adequate decontamination approaches. Another important component in fungal spore resistance is DNA repair. When damage occurs, several pathways can be activated: nucleotide excision repair (NER), mismatch repair (MMR), homologous recombination (HR), or non-homologous end-joining (NHEJ) recombination (Sinha and Hader, 2002). A. niger strains deficient in NHEJ $(\triangle k u s A)$ are widely used to generate mutant strains, but it was also shown that these strains are more sensitive to UV and X-ray irradiation (Meyer et al., 2007).

When considering fungal contamination in indoor habitats, the ability to colonize is not only dependent on spore survival, but also on the ability for spores to adhere to a surface, germinate, and grow. Germination and hyphal growth are established through polarized growth (Kwon et al., 2011, 2013). In $A$. niger, the stabilization of polarity axes during germination is dependent on the Rho GTPase RacA. A racA deletion displays a hyperbranching phenotype which results in compact colonies (Kwon et al., 2011). Spore adhesion to surfaces is facilitated by proteins in the cell wall that help fungi to grow on a wide-range of substrates (e.g., from quartz used on 
windows to silicone and polycarbonate used in medical/scientific instruments) (Makimura et al., 2001; Mora et al., 2016, 2019). Furthermore, fungal growth is surface-associated, which can induce biocorrosion. In fact, fungal-induced biocorrosion has led to major problems in spacecraft safety such as those in the Mir space station (Klintworth and Reher, 1999; Novikova et al., 2006). Understanding whether polar growth impacts spore revival and subsequent surface-associated growth is important to better control fungal contamination in the spaceflight context.

For these reasons, understanding whether A. niger spores resist to space radiation, and to what extent, will be crucial to assess both the risks and opportunities of fungal spore survival during space travel. This study has assessed $A$. niger spore survival to different types of space radiation (X-rays, cosmic radiation and $\mathrm{UV}-\mathrm{C})$. Three mutant strains were included to elucidate the role of pigmentation $(\triangle f w n A)$ and DNA repair $(\Delta k u s A)$ on spore resistance to radiation. In addition, high radiation doses were tested to assess the limits of resistance of fungal spores and their survival potential during long-term space travel. In addition, a fourth strain deficient in polar growth $(\triangle \mathrm{rac} A)$ was tested to assess the impact of UV-C treatment in spore revival and consequent ability for surface colonization.

\section{MATERIALS AND METHODS}

\section{Strains and Media}

Aspergillus niger wild-type (N402) and three mutant strains with defects in pigmentation $(\triangle f n w A)$, DNA repair $(\triangle k u s A)$, and polar growth control $(\triangle \mathrm{racA})$, were used in this study and are listed in Table 1. A. niger spores were harvested from 3-day-old cultures incubated on complete medium (CM) at

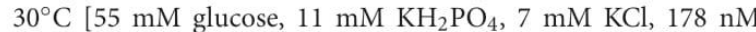
$\mathrm{H}_{3} \mathrm{BO}_{3}, 2 \mathrm{mM} \mathrm{MgSO}$, $76 \mathrm{nM} \mathrm{ZnSO}, 70 \mathrm{mM} \mathrm{NaNO}$, $6.2 \mathrm{nM} \mathrm{Na}_{2} \mathrm{MoO}_{4}, 18 \mathrm{nM} \mathrm{FeSO}, 7.1 \mathrm{nM} \mathrm{CoCl}, 6.4 \mathrm{nM}$ $\mathrm{CuSO}_{4}, 25 \mathrm{nM} \mathrm{MnCl}_{2}, 174 \mathrm{nM}$ EDTA, 0.5\% (w/v) yeast extract and $0.1 \%(\mathrm{w} / \mathrm{v})$ casamino acids] by flooding the agar plates with saline solution $(0.9 \% \mathrm{NaCl})$ and harvesting the spores using a cotton stick. Spore suspensions were filtered using Miracloth (Millipore) to remove hyphal fragments and were kept at $4^{\circ} \mathrm{C}$. Counting was done using a Neubauer chamber. All experiments were performed with spore suspensions not older than 2 weeks. Viability assays were done using minimal

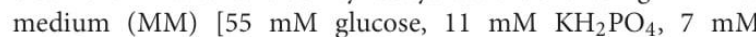
$\mathrm{KCl}, 178 \mathrm{nM} \mathrm{H}_{3} \mathrm{BO}_{3}, 2 \mathrm{mM} \mathrm{MgSO} 4,76 \mathrm{nM} \mathrm{ZnSO} 4,70 \mathrm{mM}$ $\mathrm{NaNO}_{3}, 6.2 \mathrm{nM} \mathrm{Na} \mathrm{MoO}_{4}, 18 \mathrm{nM} \mathrm{FeSO}, 7.1 \mathrm{nM} \mathrm{CoCl}$, $6.4 \mathrm{nM} \mathrm{CuSO}_{4}, 25 \mathrm{nM} \mathrm{MnCl}_{2}, 174 \mathrm{nM}$ EDTA]. For MM or $\mathrm{CM}$ agar plates, $15 \mathrm{~g}$ agar was added per liter (adapted from Carvalho et al., 2010).

\section{X-Ray Radiation Exposure}

Spores of $A$. niger strains were exposed to X-ray radiation in PCR tubes (Brand), each filled with $100 \mu \mathrm{l}$ of saline solution $(0.9 \%$ $\mathrm{NaCl}$ ) at a concentration of $10^{7}$ spores $/ \mathrm{ml}$. This concentration was chosen after testing the effect of initial spore concentration (inoculum) on survivability toward X-rays (Supplementary
Figure 1). Radiation exposure was performed using the RS225 $\mathrm{X}$-ray machine (Gulmay Medical Systems, Camberley, Surrey, United Kingdom) operated without filter at $200 \mathrm{kV}$ and $15 \mathrm{~mA}$ which allowed exposure of high doses in a short amount of time. Dose rate, in Gy/min, was determined using the UNIDOS webline with an ionization chamber type TM30013 (PTW, Freiburg, Germany). For each desired dose, the sample exposure time was adjusted given that distance and dose rate were kept constant. X-ray exposure time was calculated as follows:

$$
t(\min )=\frac{R(\mathrm{~Gy})}{d(\mathrm{~Gy} / \mathrm{min})}
$$

where $t=$ time (in minutes); $R=$ desired radiation dose (in Gy), $d$ (dosimeter value in $\mathrm{Gy} / \mathrm{min}$ ). Samples were exposed to 50, $100,250,500$, and $1000 \mathrm{~Gy}$. Given that the average dose rate was $\sim 20 \mathrm{~Gy} / \mathrm{min}$, the maximum time a sample was exposed to $\mathrm{X}$-ray radiation was $50 \mathrm{~min}$ (corresponding to $1000 \mathrm{~Gy}$ ). To test irradiation of dried spores, $25 \mu \mathrm{l}$ of a spore suspension (to a total of $10^{7}$ spores per PCR tube) either in water $\left(\mathrm{H}_{2} \mathrm{O}\right)$ or in saline solution $(0.9 \% \mathrm{NaCl})$ was placed in PCR tubes, which were left to air-dry overnight on the bench $\left(22^{\circ} \mathrm{C}\right)$ before irradiation. After irradiation, the spores were suspended in $100 \mu \mathrm{l}$ of water $\left(\mathrm{H}_{2} \mathrm{O}\right)$ or saline solution $(0.9 \% \mathrm{NaCl})$. Radiation exposure included at least three biological replicates per strain and was performed two independent times $(n=6)$. Viability was determined by colony forming units (CFUs) (see section "Viability Assay").

\section{Cosmic Radiation Exposure}

Spores were exposed to helium- and iron-ions (two components of cosmic radiation) in PCR tubes (Brand) each filled with $100 \mu \mathrm{l}$ of saline solution $(0.9 \% \mathrm{NaCl})$ at a concentration of $10^{7}$ spores $/ \mathrm{ml}$. PCR tubes were placed inside Petri dishes stacked together inside plastic bags and placed directly facing the ion beam. Samples were exposed to 10, 100, 250, and 500 Gy. Non-irradiated controls were left at room temperature. Viability was calculated by CFU (see section "Viability Assay"). Radiation exposure included three biological replicates per strain $(n=3)$. Cosmic radiation exposure was performed at the Heavy Ion Medical Accelerator (HIMAC) facility at the National Institute of Radiological Sciences (NIRS) in Chiba, Japan. The helium-ion beam had an energy of $150 \mathrm{MeV} / \mathrm{n}$ and linear energy transfer (LET) of $2.2 \mathrm{keV} / \mu \mathrm{m}$. The iron-ion beam had an energy of $500 \mathrm{MeV} / \mathrm{n}$ and LET of $200 \mathrm{keV} / \mu \mathrm{m}$. Each tested dose was adjusted by exposure time, since beam energy and LET was kept constant.

\section{UV-C Radiation Exposure}

Spores of $A$. niger were exposed to UV radiation in Petri dishes with an initial concentration of $10^{6}$ spores $/ \mathrm{ml}$ in $15 \mathrm{ml}$ of saline solution $(0.9 \% \mathrm{NaCl})$. The concentration of $10^{6}$ spores $/ \mathrm{ml}$ ensures a spore monolayer and prevents survival due to shielding by the spores themselves. The UV lamp (MagneTel, Menomonee Falls, WI, United States) was used a UV-C monochromatic wavelength of $254 \mathrm{~nm}$. During irradiation, magnetic stirrers continuously mixed the spore suspension in order to avoid 
TABLE 1 | Aspergillus niger strains used in the study.

\begin{tabular}{|c|c|c|c|c|}
\hline Name & Strain & $\begin{array}{l}\text { Relevant } \\
\text { genotype }\end{array}$ & Description & References \\
\hline Wild-type & $\mathrm{N} 402$ & & $\begin{array}{l}\text { Wild-type strain capable of DNA repair and pigment formation which give } \\
\text { black-colored spores }\end{array}$ & Bos et al., 1988 \\
\hline Color mutant & MA93.1 & $\triangle f w n A$ & Loss of pigment, due to lack of polyketide synthase results in fawn-colored spores & Jorgensen et al., 2011 \\
\hline NHEJ mutant & MA78.6 & $\triangle k u s A$ & Inactive in NHEJ pathway and thus impaired in DNA repair & Meyer et al., 2007 \\
\hline Polar growth mutant & MA80.1 & $\Delta k u s A, \Delta r a c A$ & Inactive in NHEJ pathway and polar growth control & Kwon et al., 2011 \\
\hline
\end{tabular}

mutual shielding of the spores. The radiation dose $\left[\mathrm{J} / \mathrm{m}^{2}\right]$ was adjusted through exposure time, since the height of the UV lamp was kept constant. For that, UV fluence was determined using the dosimeter (UVP UVX radiometer), and exposure time was adjusted for each sample to reach each desired UV dose (i.e., 150, $250,500,1000,2000,3000$, and $4000 \mathrm{~J} / \mathrm{m}^{2}$ ). UV-C exposure time was calculated as follows:

$$
t(\mathrm{~s})=\frac{R\left(\mathrm{~J} / \mathrm{m}^{2}\right) \times 100}{d\left(\mu \mathrm{W} / \mathrm{cm}^{2}\right)}
$$

where $t=$ time (in seconds); $R=$ desired radiation dose (in $\left.\mathrm{J} / \mathrm{m}^{2}\right), d$ (dosimeter value for UV fluence, in $\mu \mathrm{W} / \mathrm{cm}^{2}$ ). After each time point, corresponding to a certain dose, $100 \mu \mathrm{l}$ of sample were taken in triplicate from the spore suspension and transferred into PCR tubes. Viability was calculated by CFU (see section "Viability Assay"). Radiation exposure included at least three biological replicates per strain and was performed two independent times $(n=6)$.

\section{Oxidative Stress Assay}

Oxidative stress resistance toward hydrogen peroxide $\left(\mathrm{H}_{2} \mathrm{O}_{2}\right)$ was measured using a protocol adapted from Riesenman and Nicholson (2000). Spores of A. niger were diluted to a final concentration of $10^{8}$ spores $/ \mathrm{ml}$ in saline solution $(0.9 \% \mathrm{NaCl})$, $833 \mu \mathrm{l}$ of which were placed in a $5 \mathrm{ml}$ tube (Eppendorf). Afterward $167 \mu \mathrm{l}$ of $30 \% \mathrm{H}_{2} \mathrm{O}_{2}$ were added (Sigma-Aldrich). The spore- $\mathrm{H}_{2} \mathrm{O}_{2}$ suspension was gently mixed at RT $\left(\sim 22^{\circ} \mathrm{C}\right)$ and incubated for up to $15 \mathrm{~min}$ in a final concentration of $5 \%$ $\mathrm{H}_{2} \mathrm{O}_{2} .30 \mu \mathrm{l}$ of the suspension were taken at different time points and diluted $1: 10$ in saline solution $(0.9 \% \mathrm{NaCl})$ with bovine catalase $(100 \mu \mathrm{g} / \mathrm{ml})$ to stop the oxidation reaction. Samples were serially diluted and used to determine viability (see section "Viability Assay"). This assay included three biological replicates per tested strain, and was performed two independent times $(n=6)$.

\section{Viability Assay}

Viability of $A$. niger spores was determined by their ability to form colonies after exposure to the tested environments. Samples were serially diluted up to $10^{-8}$ using a 96-well plate, each well with a total volume of $300 \mu \mathrm{l}$. To count the CFUs, $20 \mu \mathrm{l}$ of each dilution was plated out in triplicate on $1 / 8$ of a Petri dish with MM agar, containing Triton X-100 (0.05\%) to facilitate counting. The plates were incubated for 2 days at $30^{\circ} \mathrm{C}$ before the colonies were counted. This allowed calculation of the survival fraction ratio (N/NO, in which $\mathrm{N}$ is the number of $\mathrm{CFU}$ of the treated samples and N0 that of the controls). To analyze the microscopic morphology, previously irradiated samples were diluted 100 -fold before plating $10 \mu \mathrm{l}$ on a MM agar plate. These were incubated for 1 day at $30^{\circ} \mathrm{C}$, after which a Zeiss microscope (Axio Imager.M2) was used to take images of representative areas on the agar plate for further analysis (Supplementary Figure 2). Viability assays included three biological replicates per tested strain and were performed at two independent times $(n=6)$.

\section{Crystal Violet Assay}

To assess fungal biofilm formation, i.e., surface-associated growth with production of extracellular matrix, a crystal violet assay was performed, adapted from Mowat et al. (2007). In a 96well plate each well contained $100 \mu \mathrm{l}$ spore suspension (to a total concentration of $10^{5}$ spores $/ \mathrm{ml}$ per well), $100 \mu \mathrm{l} \mathrm{MM}$, and $100 \mu \mathrm{l}$ of distilled $\mathrm{H}_{2} \mathrm{O}\left(\mathrm{dH}_{2} \mathrm{O}\right)$ (the controls contained an additional $100 \mu \mathrm{l} \mathrm{MM}$ instead of $100 \mu \mathrm{l}$ spore suspension) and were incubated for $48 \mathrm{~h}$ at $30^{\circ} \mathrm{C}$. After incubation, the wells were washed three times with $\mathrm{dH}_{2} \mathrm{O}$, and $300 \mu \mathrm{l}$ of crystal violet $(0.5 \%)$ were added to each well to stain the surface-associated biomass (crystal violet stains hyphae and extracellular matrix that do not detach after washing). Excess staining was removed by washing with $\mathrm{dH}_{2} \mathrm{O}$. De-staining was carried out by adding $300 \mu \mathrm{l}$ of $95 \%$ ethanol. The absorbance of the ethanol-crystal violet solution was measured at $570 \mathrm{~nm}$. The higher the absorbance value the greater the quantity of biological material. The absorbance was evaluated using the VICTOR Nivo Multimode Microplate Reader (PerkinElmer, Waltham, MA, United States). The assay included eight biological replicates per tested strain and was performed twice $(n=16)$

\section{Data Analysis}

Student's $t$-test was performed to analyze the significance between individual data points where a two-tailed $p$-value $\leq 0.05$, was considered significant. Error bars as standard error. Linear regression on survival fraction data was used to calculate the lethal dose for $90 \%$ of the population ( $\mathrm{LD}_{90}$ values) - which is the same as $\mathrm{D}_{10}$ values ( $10 \%$ survivability).

\section{RESULTS}

\section{Aspergillus niger Spore Resistance Toward X-Ray Radiation}

Spores from the wild-type, color mutant $(\triangle f w n A)$ and NHEJ mutant $(\triangle k u s A)$ strains were exposed to different X-ray doses 
TABLE 2 | Lethal dose (LDgo) values for Aspergillus niger spores irradiated with X-rays under different space-relevant conditions.

\begin{tabular}{|c|c|c|c|c|}
\hline Strain & $0.9 \% \mathrm{NaCl}$ & $\mathrm{H}_{2} \mathrm{O}$ & Air-dried $(0.9 \% \mathrm{NaCl})$ & Air-dried $\left(\mathrm{H}_{2} \mathrm{O}\right)$ \\
\hline Wild-type & $366\left(R^{2}=0.97\right)$ & $362\left(R^{2}=0.96\right)$ & $187\left(R^{2}=0.99\right)$ & $204\left(R^{2}=0.99\right)$ \\
\hline Color mutant $(\triangle f w n A)$ & $353\left(R^{2}=0.98\right)$ & $306\left(R^{2}=0.99\right)$ & $175\left(R^{2}=0.99\right)$ & $185\left(R^{2}=0.99\right)$ \\
\hline NHEJ mutant ( $\triangle$ kusA) & $57\left(R^{2}=0.93\right)$ & $55\left(R^{2}=0.92\right)$ & $35\left(R^{2}=0.99\right)$ & $45\left(R^{2}=0.95\right)$ \\
\hline
\end{tabular}

Data reported as $L D_{90}$ values - dose of radiation treatment leading to a $90 \%$ inactivation of the initial CFU (same as $D_{10}$ values). Values in Gray (Gy).

TABLE 3 | Lethal dose (LDgo) values for Aspergillus niger spores irradiated with different types of ionizing radiation.

\begin{tabular}{lccc}
\hline Strain & X-rays (Gy) & Helium-ion (Gy) & Iron-ion (Gy) \\
\hline Wild-type & $366\left(R^{2}=0.97\right)$ & $506\left(R^{2}=0.98\right)$ & $112\left(R^{2}=0.99\right)$ \\
Color mutant $(\triangle f w n A)$ & $353\left(R^{2}=0.98\right)$ & $567\left(R^{2}=0.96\right)$ & $112\left(R^{2}=0.99\right)$ \\
NHEJ mutant $(\triangle$ kuSA) & $57\left(R^{2}=0.93\right)$ & $55\left(R^{2}=0.99\right)$ & $50\left(R^{2}=0.99\right)$
\end{tabular}

Data reported as $L D_{90}$ values - dose of radiation treatment leading to a $90 \%$ inactivation of the initial CFU (same as $D_{10}$ values).

(up to $1000 \mathrm{~Gy}$ ) in saline solution. The lethal dose required to inactivate $90 \%$ of the spores was similar for both wild-type $\left(\mathrm{LD}_{90}=366 \mathrm{~Gy}\right)$ and $\Delta f w n A$ strains $\left(\mathrm{LD}_{90}=353 \mathrm{~Gy}\right)$. After the maximum tested radiation dose of $1000 \mathrm{~Gy}$, the $\Delta f w n A$ strain demonstrated no significant differences in survival when compared with the wild-type $\left(5.3 \times 10^{3} \pm 1.3 \times 10^{3} \mathrm{CFU} / \mathrm{ml}\right.$, versus $1 \times 10^{4} \pm 3.2 \times 10^{3} \mathrm{CFU} / \mathrm{ml}$ ). In contrast, at $500 \mathrm{~Gy}$, $\triangle k u s A$ was shown to be significantly more sensitive to $\mathrm{X}$-ray radiation $\left(1.25 \times 10^{4} \pm 1.1 \times 10^{3} \mathrm{CFU} / \mathrm{ml}\right.$ with a $\mathrm{LD}_{90}$ of $\left.57 \mathrm{~Gy}\right)$, when compared to the wild-type $\left(5 \times 10^{6} \pm 8.7 \times 10^{5} \mathrm{CFU} / \mathrm{ml}\right)$ $(p=0.000)$. No CFU were detected above $500 \mathrm{~Gy}$ for $\Delta k u s A$. Survivability and $\mathrm{LD}_{90}$ values of the strains toward $\mathrm{X}$-ray radiation, in all tested conditions, are shown summarized in Table 2 .

\section{Air-Drying of $\boldsymbol{A}$. niger Spores Reduces Their Resistance Toward X-Ray Radiation}

Survivability of air-dried A. niger spores was compared with survivability of wet spores (both in water and saline solution). The highest X-ray dose at which there were detectable colonies was $500 \mathrm{~Gy}$ for both wild-type and $\triangle f w n A$ strains, and $250 \mathrm{~Gy}$ for $\Delta k u s A$. Results show that survivability of dried spores was decreased in comparison to wet spores (both in water and saline solution) in all tested strains (Figure 1). At $500 \mathrm{~Gy}$, wild-type spores in water $\left(4.9 \times 10^{6} \pm 1.1 \times 10^{6} \mathrm{CFU} / \mathrm{ml}\right)$ survive better than dried spores from water $\left(4.6 \times 10^{4} \pm 7.2 \times 10^{3} \mathrm{CFU} / \mathrm{ml}\right)$ $(p=0.001)$. The same is seen for spores in saline solution where wet spores $\left(5 \times 10^{6} \pm 8.7 \times 10^{5} \mathrm{CFU} / \mathrm{ml}\right)$ survived better than dried spores from saline solution $\left(8.2 \times 10^{3} \pm 1.2 \times 10^{3} \mathrm{CFU} / \mathrm{m}\right)$ ( $p=0.000)$ (Table 2). When comparing water- versus salinedried spores, wild-type spores dried from saline solution $\left(8.2 \times 10^{3} \pm 1.2 \times 10^{3} \mathrm{CFU} / \mathrm{ml}\right)$ survive significantly less than spores dried from water $\left(4.6 \times 10^{4} \pm 7.2 \times 10^{3} \mathrm{CFU} / \mathrm{ml}\right)$ $(p=0.000)$. Loss of pigmentation did not affect resistance to desiccation (0 Gy) ( $p=0.6$ ); or radiation resistance (at $500 \mathrm{~Gy}$ ) when spores were air-dried from saline solution $(p=0.4)$. However, loss of pigmentation decreased spore survival in irradiated spores dried in water: at $500 \mathrm{~Gy}, \Delta f w n A$ spores were significantly more sensitive $\left(5.2 \times 10^{3} \pm 1.2 \times 10^{3} \mathrm{CFU} / \mathrm{ml}\right)$ than wild-type spores $\left(4.6 \times 10^{4} \pm 7.2 \times 10^{3} \mathrm{CFU} / \mathrm{ml}\right)(p=0.002)$.

\section{Aspergillus niger Spores Resistance Toward Cosmic Radiation}

The effect of cosmic radiation on the survival of $A$. niger was tested by irradiating spores with helium- and ironions. Spores from the wild-type, color mutant $(\triangle f w n A)$ and NHEJ mutant $(\triangle k u s A)$ strains were exposed to up to $500 \mathrm{~Gy}$ of helium- and iron-ions. At $500 \mathrm{~Gy}$, wildtype spores survived less when irradiated with iron-ions $\left(4.7 \times 10^{2} \pm 6.01 \times 10^{1} \mathrm{CFU} / \mathrm{ml}\right)$ than when irradiated with $\mathrm{X}$-rays $\left(5 \times 10^{6} \pm 8.7 \times 10^{5} \mathrm{CFU} / \mathrm{ml}\right)(p=0.06)$, or heliumions $\left(8.7 \times 10^{6} \pm 1.9 \times 10^{6} \mathrm{CFU} / \mathrm{ml}\right)(p=0.01)$ (Table 3$)$. The same trend holds for $\triangle$ fwnA spores at $500 \mathrm{~Gy}$, where survival toward iron-ions $\left(4.3 \times 10^{2} \pm 1.9 \times 10^{2} \mathrm{CFU} / \mathrm{ml}\right)$ was decreased in comparison to both X-rays $\left(3.4 \times 10^{6} \pm 5.4 \times 10^{5} \mathrm{CFU} / \mathrm{ml}\right)$ $(p=0.004)$ and helium-ions $\left(1.2 \times 10^{7} \pm 2.0 \times 10^{6} \mathrm{CFU} / \mathrm{ml}\right)$ $(p=0.004)$ (Figures 1, 2 and Table 3 ). This is consistent with the fact that helium-ions are lighter elements in cosmic radiation whereas iron-ions are heavier particles, which cause greater damage to cells (Cucinotta and Durante, 2006). At $250 \mathrm{~Gy}$, survival of $\Delta k u s A$ spores was significantly reduced when irradiated with iron-ions $\left(1.9 \times 10^{3} \pm 9.9 \times 10^{1} \mathrm{CFU} / \mathrm{ml}\right)$ than when irradiated with X-rays $\left(4.7 \times 10^{2} \pm 6.01 \times 10^{1} \mathrm{CFU} / \mathrm{ml}\right)$ $(p=0.02)$, with no colony formation being observed above 250 Gy of cosmic radiation (Figure 2). Exposure to heliumand iron-ions (cosmic radiation) showed that both wild-type and $\Delta k u s A$ spores were able to germinate after exposure to 250 and $500 \mathrm{~Gy}$, respectively (Supplementary Figure 2), suggesting that there is a greater level of resistance if germination would be considered instead of colony forming ability. Since $\Delta f w n A$ spores do not show reduced resistance toward X-ray radiation (and subsequent ROS) in comparison to the wild-type strain, we tested whether pigmentation is involved in protecting the spore from $\mathrm{H}_{2} \mathrm{O}_{2}$-induced oxidative-stress by incubating the spores up to $15 \mathrm{~min}$ in $5 \% \mathrm{H}_{2} \mathrm{O}_{2}$ (Figure 3). Both the wild-type and color mutant strains decreased in survival when incubated with $\mathrm{H}_{2} \mathrm{O}_{2}$. The survival of $\triangle$ fwnA spores after $15 \mathrm{~min}$ was lower than that of the wild-type, with the $\mathrm{LD}_{90}$ value for the 


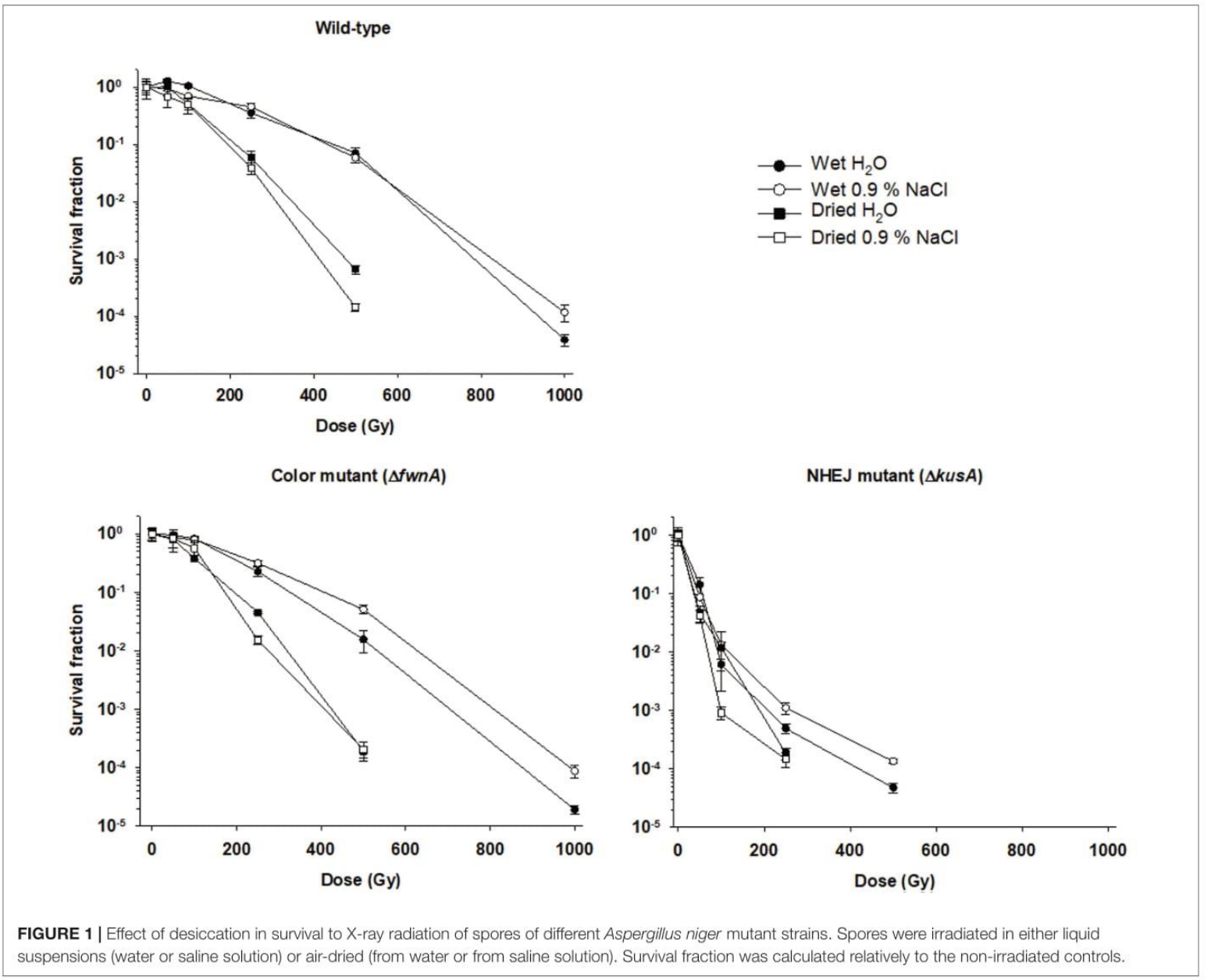

wild-type strain ( $5 \mathrm{~min}$ ) being higher than that of the $\Delta f w n A$ (3.1 min) (Figure 3).

\section{Aspergillus niger Spores Are Highly Resistant Toward UV-C Radiation}

To investigate the impact of UV radiation on A. niger spore survivability, spore suspensions with $10^{6}$ spores $/ \mathrm{ml}$ (spore monolayer) were exposed to $0-4000 \mathrm{~J} / \mathrm{m}^{2} \mathrm{UV}-\mathrm{C}$ radiation $(254 \mathrm{~nm})$. At the highest tested dose of $4000 \mathrm{~J} / \mathrm{m}^{2}$, both the wild-type $\left(7.1 \times 10^{2} \pm 4.3 \times 10^{2} \mathrm{CFU} / \mathrm{ml}\right)$ and polar growth mutant $\left(4.2 \times 10^{1} \pm 2.5 \times 10^{1} \mathrm{CFU} / \mathrm{ml}\right)$ demonstrated high survival (Figure 4). No CFU were detected for $\Delta k u s A$ at $4000 \mathrm{~J} / \mathrm{m}^{2}$. All tested strains were able to cope with $3000 \mathrm{~J} / \mathrm{m}^{2}$ of UV-C exposure. At $3000 \mathrm{~J} / \mathrm{m}^{2}$ wild-type spores $\left(1.3 \times 10^{3} \pm 6.8 \times 10^{2} \mathrm{CFU} / \mathrm{ml}\right)$ and $\triangle \mathrm{racA}$ spores $\left(2.3 \times 10^{2} \pm 1.3 \times 10^{2} \mathrm{CFU} / \mathrm{ml}\right)$ displayed high survivability; whereas $\triangle f w n A$ spores $\left(6.3 \times 10^{1} \pm 3.7 \times 10^{1} \mathrm{CFU} / \mathrm{ml}\right)$ and $\Delta$ kusA spores $\left(1.2 \times 10^{2} \pm 0.1 \times 10^{1} \mathrm{CFU} / \mathrm{ml}\right)$ displayed low survivability. The UV-C dose required to eliminate $90 \%$ of the wild-type spores $\left(\mathrm{LD}_{90}\right)$ was $1038 \mathrm{~J} / \mathrm{m}^{2} . \mathrm{LD}_{90}$ of $\triangle \mathrm{racA}$ spores was $826 \mathrm{~J} / \mathrm{m}^{2}$, of $\Delta k u s A$ spores was $580 \mathrm{~J} / \mathrm{m}^{2}$, and for $\Delta f w n A$ spores it was $512 \mathrm{~J} / \mathrm{m}^{2}$. The data also shows that deletion of $\mathrm{racA}$ increases survival toward UV-C (Figure 4), in all tested doses.

\section{Defect in Both NHEJ and Polar Growth Decreases A. niger Biofilm Formation}

When assessing $A$. niger biofilm formation (quantified as amount of surface-adhered biomass detected in the well after washing), a defect in pigmentation $(\triangle f w n A)$ decreased biofilm formation by $23 \%(p=0.02)$; a defect in the NHEJ pathway $(\Delta k u s A)$ decreased biofilm formation by $25 \%(p=0.01)$; and a defect in both NHEJ and polar growth ( $\triangle k u s A, \triangle r a c A$ ) showed a decrease of $49 \%$ $(p=0.007)$ (Figure 5B). When assessing the effect of UV-C radiation in wild-type biofilm formation, we found that biomass decreased as doses increased up to $4000 \mathrm{~J} / \mathrm{m}^{2}$ (Figure 5A). Thus, because the UV-C $L_{90}$ value for wild-type spores was $1000 \mathrm{~J} / \mathrm{m}^{2}$, we tested biofilm formation after treatment with $1000 \mathrm{~J} / \mathrm{m}^{2} \mathrm{UV}$ C for all strains. UV-C treatment led to $81 \%$ reduction in biofilm 

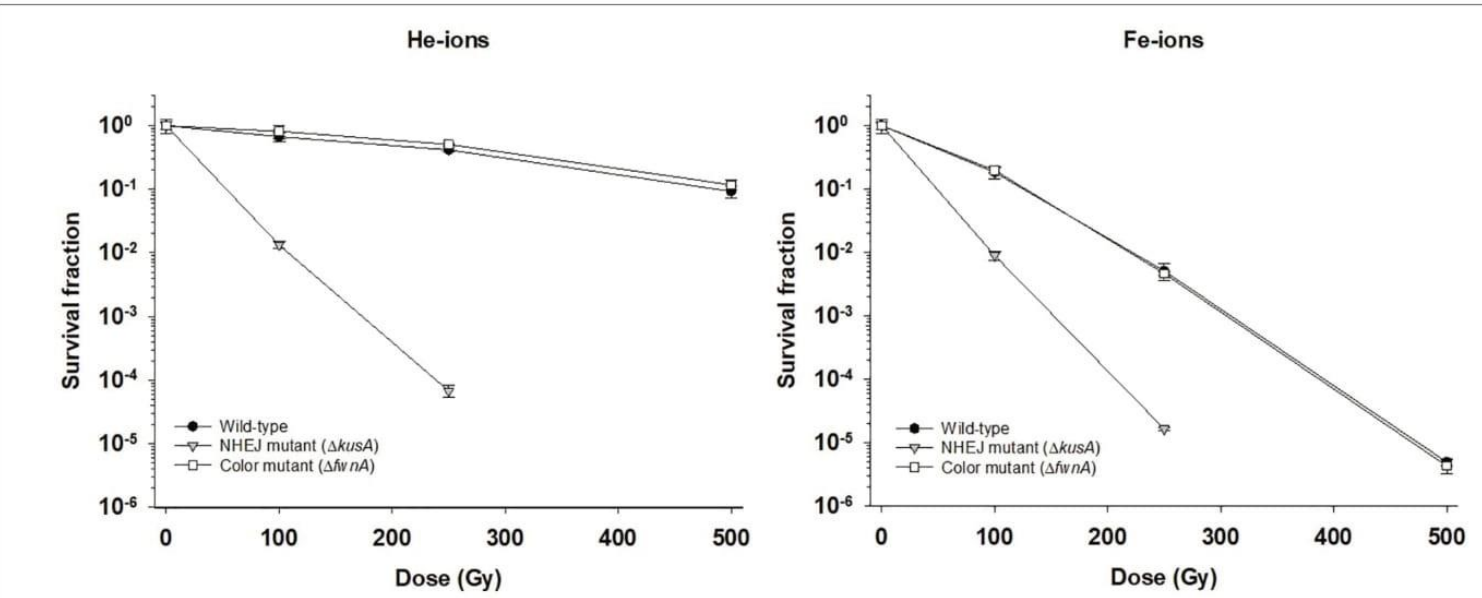

FIGURE 2 | Effect of cosmic radiation (helium-ions - left; and iron-ions - right) on survival of spores of different Aspergillus niger strains. Survival fraction was calculated relatively to the non-irradiated controls.

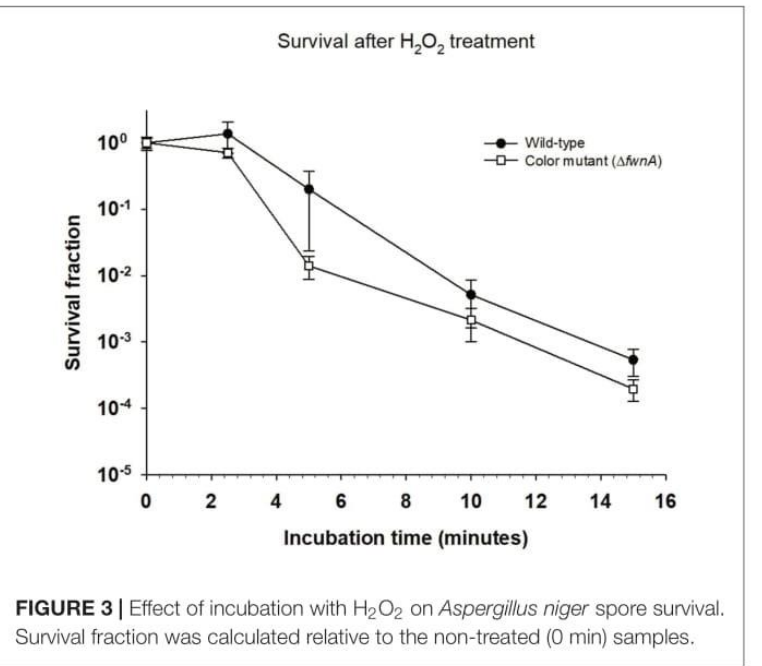

formation for wild-type spores $(p=0.04), 97 \%$ reduction in $\triangle$ fnwA spores, $(p=0.001), 82 \%$ in $\triangle k u s A$ spores $(p=0.001)$, and $94 \%$ in $\triangle$ racA $\triangle$ kusA spores, $(p=0.005)$ (Figure 5B).

\section{DISCUSSION}

\section{Air Drying A. niger Spores Reduces Their Resistance Toward X-Ray Radiation}

Spore survival toward X-rays was tested in both air-dried and liquid conditions (a comparison is provided in Table 4). Results show that air-dried spores have lower survival rates than spores irradiated in liquid suspension (either in water, or in saline solution) (Figure 1). This result is unexpected, as the presence

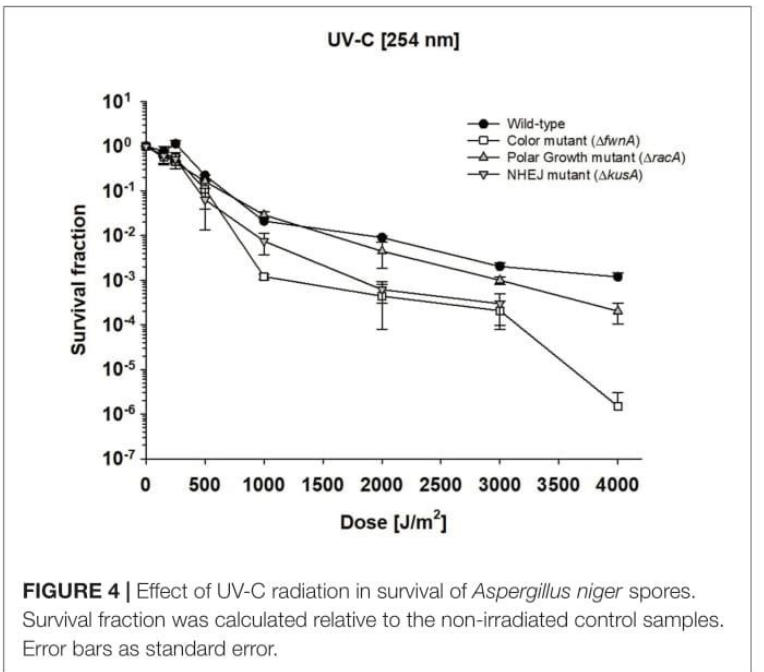

of water is known to decrease radiation resistance via ROS formation. Nevertheless, A. niger spores have previously been shown to have increased radiation sensitivity when vacuum-dried and irradiated in air compared to wet spores irradiated in air (Silverman et al., 1967). However, the same study reported that vacuum-dried spores irradiated in vacuum were found to be more resistant to radiation, which implies that space vacuum desiccation might increase A. niger spore resilience (Silverman et al., 1967). Interestingly, a study assessing the impact of water in radiation resistance of yeast reports that small amounts of water substantially increases radiation sensitivity (Hutchinson et al., 1957). Thus, we consider the possibility that drying the spores overnight might have not been enough to retrieve all water from the spore suspension. 

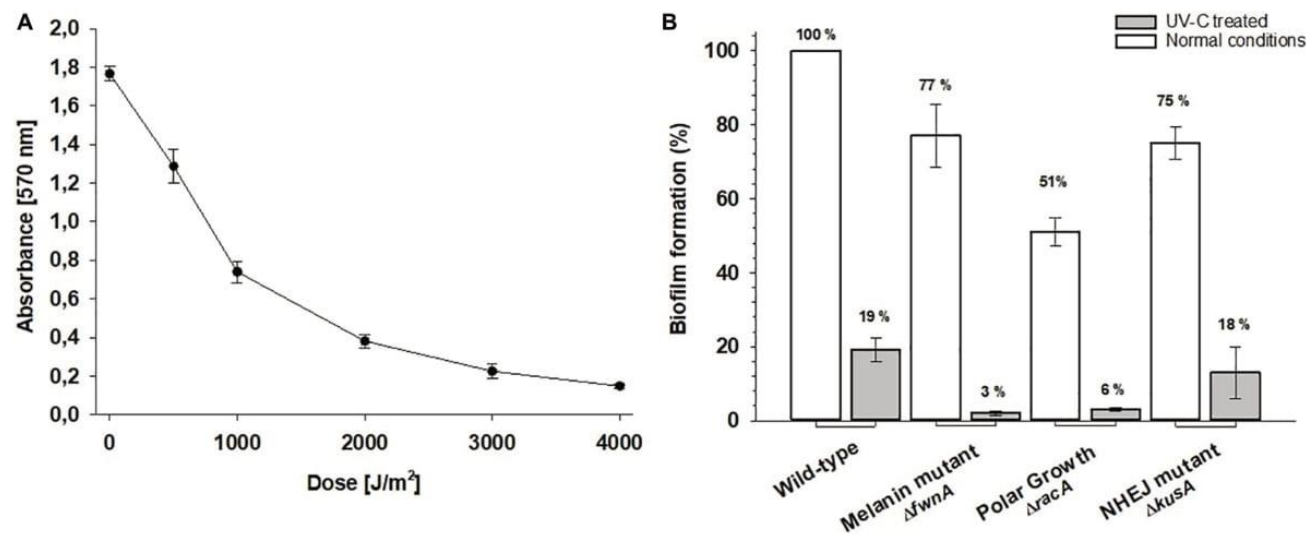

FIGURE 5 | (A) Biofilm formation of Aspergillus niger wild-type after 0 to $4000 \mathrm{~J} / \mathrm{m}^{2} \mathrm{UV}-\mathrm{C}(254 \mathrm{~nm})$. Absorbance values are proportional to the amount of detected biomass. (B) Effect of UV-C treatment on A. niger biofilm formation. The biofilm formation is shown for normal conditions (white bars) and after treatment with $1000 \mathrm{~J} / \mathrm{m}^{2}$ UV-C (gray bars).

TABLE 4 | Lethal dose $\left(L D_{90}\right)$ values for Aspergillus niger spores in comparison to different organisms in response to UV-C radiation (254 nm) and X-ray radiation. Values for cells/spores in suspension (wet), irradiated in air.

\begin{tabular}{lclcl}
\hline Strain & UV-C $\left(\mathbf{J} / \mathbf{m}^{2}\right)$ & References & X-rays (Gy) & References \\
\hline Aspergillus niger (spores) & 1038 & This study & 366 & This study \\
Bacillus subtilis (spores) & 100 & Newcombe et al., 2005 & 857 & Moeller et al., 2014 \\
Deinococcus radiodurans (cells) & 656 & Bauermeister et al., 2009 & ca. 8000 & Moseley and Laser, 1965; Driedger et al., 1970
\end{tabular}

Data reported as $L D_{90}$ values - dose of radiation treatment leading to a $90 \%$ inactivation of the initial CFU (same as $D_{10}$ values).

Additionally, pigmentation did not have an effect on desiccation resistance (at $0 \mathrm{~Gy}$ ), which is consistent with previous findings concerning the role of pigmentation in fungal survival to low water activity (Segers et al., 2018). When irradiating air-dried spores with $500 \mathrm{~Gy}$, there was no difference between wild-type and color mutant for spores dried in saline. However, the color mutant had increased sensitivity when spores were dried in water. Thus, to fully understand the effect of desiccation (air- or vacuum-induced) on fungal spore resistance to extreme radiation further studies are required.

\section{High-Versus Low-LET Response in A. niger Spores}

The biological effectiveness of radiation in a given biological sample is dependent on the LET. This means that equal doses of different types of radiation can induce different types of damage, and thus different cellular responses (Moeller et al., 2017). With this, previous studies with animals suggest that indirect damage is the main biological effect of low-LET radiations (such as X-rays or helium-ions), whereas direct damage is the main biological effect of high-LET radiation (such as iron-ions) (Kennedy, 2014). In contrast, the current study suggests that direct damage, in the form of DNA double-strand breaks, is induced by low-LET radiation exposure (X-rays and helium-ions), given that $A$. niger spore survivability was highly dependent on the NHEJ pathway $(\triangle k u s A)$. Additionally, $\Delta k u s A$ spores showed similar survival rates toward both high-LET iron-ion radiation (Figure 2) and
low-LET X-ray radiation (Figure 1). This implies that radiation with iron-ions promotes additional cellular damages besides double-strand breaks, possibly as indirect damage in the form of ROS generation. Multiple experiments have shown similar results in yeasts exposed to ion radiation (Ikpeme et al., 1995; Kiefer et al., 2002). Conversely, a study with the filamentous fungus Neurospora crassa, showed that an NHEJ-deficient strain had $\sim 80 \%$ survival after 100 Gy of high-LET carbon-ion irradiation (Ma et al., 2013), whereas the NHEJ-deficient A. niger strain tested in the current study demonstrated survival of only $\sim 17 \%$ after $100 \mathrm{~Gy}$ of low-LET helium-ion radiation (a lighter element than carbon). Nevertheless, this discrepancy in survival might be attributed to the fact that hyphal compartments of $N$. crassa can contain up to 100 nuclei, which in turn can lower the hit rate of radiation induced DNA damage (Roper et al., 2011).

\section{High Resistance of $A$. niger Spores Toward UV-C}

UV-derived decontamination methods are commonly used in modern laboratories and healthcare systems (Yang et al., 2019), with UV-C lamps used to sterilize biosafety cabinets reaching around $300 \mathrm{~J} / \mathrm{m}^{2}$ in $12.5 \mathrm{~min}$ (Meechan and Wilson, 2006). Yet, in this study $A$. niger wild-type spores demonstrated high resistance toward UV-C (Figure 4, and Supplementary Figure 3), where the lethal dose required to eliminate $90 \%$ of wild-type spores was $1038 \mathrm{~J} / \mathrm{m}^{2}$. This is significantly higher than the $\mathrm{LD}_{90}$ of other microorganisms (Table 4), and becomes particularly clear 
in comparison with previously characterized radiation-resistant organisms such as Deinococcus radiodurans $\left(\mathrm{LD}_{90}=660 \mathrm{~J} / \mathrm{m}^{2}\right)$ or B. subtilis spores $\left(\mathrm{LD}_{90}=100 \mathrm{~J} / \mathrm{m}^{2}\right)$. In a study using UV$\mathrm{C}$ to treat drinking water, $A$. niger was found to be completely inactivated after exposure to $1920 \mathrm{~J} / \mathrm{m}^{2} \mathrm{UV}-\mathrm{C}$ (Sisti et al., 2017), which is in agreement with our study $\left(\mathrm{LD}_{90}=1038 \mathrm{~J} / \mathrm{m}^{2}\right)$.

The NHEJ pathway was shown to be an important DNA repair mechanism for survival of A. niger spores after exposure to UV-C and X-ray radiation. Interestingly, previous studies with NHEJ mutants of N. crassa and Cryptococcus neoformans did not show differences in survival after UV-C irradiation when compared to the wild-type (Ninomiya et al., 2004; Goins et al., 2006). It is possible that point mutations and singlestrand breaks caused by UV radiation can be repaired by KusA independent repair mechanisms (Eckardt-Schupp and Klaus, 1999; Goldman et al., 2002). However, the higher nucleus number per hyphal compartment in N. crassa, and the capsule formation of C. neoformans spores (McFadden and Casadevall, 2001) may contribute to their increased radiation tolerance despite mutations in this repair pathway.

\section{Pigmentation as Key-Protection Against UV-C but Not X-Rays or Cosmic Radiation}

Pigments, such as melanin are involved in different cellular processes from adhesion to virulence (Eisenman and Casadevall, 2012), and are known to help cells against radiation-induced stress and ROS (Cockell and Knowland, 1999). Studies concerning the role of pigmentation in radiation resistance of fungal spores have been performed with monochromatic UV-C $(254 \mathrm{~nm})$ and pulsed light up to $1770 \mathrm{~J} / \mathrm{m}^{2}$ on inoculated agar with concentrations up to $10^{7}-10^{8}$ spores $/ \mathrm{ml}$ (Esbelin et al., 2013). The current study irradiated spore suspensions in liquid, using a concentration of $10^{6}$ spores $/ \mathrm{ml}$. This concentration was chosen to guarantee a spore monolayer and prevent selfshielding (Figure 4). As expected, pigment-deficient spores $(\triangle f w n A)$ demonstrated lower survivability after exposure to UV-C radiation. Interestingly, deficiency in pigmentation did not alter survivability after exposure to ionizing radiation (X-rays or cosmic radiation) (Figures 1, 2). This seems to contradict previous studies where melanin was shown to have a protective effect against $\mathrm{X}$-ray irradiation in the fungi C. neoformans and Cryomyces antarcticus (Pacelli et al., 2017). However, it is to note that the strain tested in the current study lacks a putative polyketide synthase which results in fawn-colored (not white) spores, which might provide sufficient amount of pigmentation to display protective properties.

Both ionizing radiation and hydrogen peroxide are known to affect cell survival through the generation of ROS. To better understand how pigment-deficient spores resist to ionizing radiation, these were incubated in hydrogen peroxide. Results show that $\triangle f w n A$ spores were more sensitive to hydrogen peroxide than wild-type spores (Figure 3). This indicates that pigmentation is involved in protecting the spore from $\mathrm{H}_{2} \mathrm{O}_{2}$-induced oxidative-stress, but not in X-ray- or cosmic radiation-induced oxidative stress. Previous studies analyzing the effect of $\mathrm{H}_{2} \mathrm{O}_{2}$ on $A$. niger spores were able to show that ROS scavenging was facilitated by increased catalase expression resistance (Angelova et al., 2005). From the results presented here, contrary to what has been suggested, pigmentation does not influence $A$. niger survival to space-like ionizing radiation.

\section{UV-C Radiation Decreases Biofilm Formation Effectively}

To address the contamination risks and possible decontamination procedures concerning $A$. niger growth in the spaceflight environment, wild-type spores were exposed to up to $4000 \mathrm{~J} / \mathrm{m}^{2}$ of UV-C and we assessed the impact of UV-C on surface-associated growth, i.e., biofilm formation (Figure 5A). Because wild-type spores were $90 \%$ inactivated at a dose of $1000 \mathrm{~J} / \mathrm{m}^{2}$, the ability for biofilm formation was assessed, for all strains, before and after treatment with $1000 \mathrm{~J} / \mathrm{m}^{2}$ UV-C. Here, one additional strain, deficient in polar growth, was included. This strain lacks the Rho GTPase RacA involved in establishing polarized tip extension via regulation of the actin filaments, which is important for proper cell wall formation in A. niger hyphae (Kwon et al., 2011, 2013). Results show that $\triangle \mathrm{racA}$ spores ability for biofilm formation was reduced by $49 \%$ before UV-C treatment, and $94 \%$ after UV-C treatment, when compared with the wild-type (Figure 5B). The underlying molecular mechanism for reduced surface-associated growth (before UV-C) might be due reduced adhesion to hydrophobic surfaces, and/or results in less spore aggregation during spore outgrowth - a hypothesis worth studying further. The reduction of surface-associated growth after exposure to radiation suggests that the function of RacA plays a role (direct or indirect) in UV-C resistance of $A$. niger. Moreover, the tested color mutant $(\triangle f w n A)$ strain also demonstrated decreased ability of surface-associated growth, both before and after UV-C treatment, which suggests the involvement of pigments in spore adhesion.

\section{CONCLUSION}

This study shows that spores of $A$. niger are extremely resistant to space radiation. Spores were able to withstand high doses of X-ray $\left(\mathrm{LD}_{90}=360 \mathrm{~Gy}\right)$, cosmic radiation (helium-ion $\mathrm{LD}_{90}=500 \mathrm{~Gy}$; and iron-ion $\mathrm{LD}_{90}=100 \mathrm{~Gy}$ ), and UV-C radiation $\left(\mathrm{LD}_{90}=1038 \mathrm{~J} / \mathrm{m}^{2}\right)$. A. niger spore resistance to UV-C is particularly interesting, given that it is even higher than that of other radiation-resistant microorganisms (e.g., D. radiodurans). Air-drying the spores made them more susceptible to X-ray radiation. Moreover, wild-type spores treated with $1000 \mathrm{~J} / \mathrm{m}^{2} \mathrm{UV}$ C were shown to have decreased biofilm formation ability $(81 \%$ reduction). It is important to note that the ionizing radiation doses used in this study (up to $1000 \mathrm{~Gy}$ ) are multiple times higher than doses expected from traveling in interplanetary space. For example, a 360-day round-trip to Mars would yield a dose of $0.66 \pm 0.12 \mathrm{~Gy}$ (Zeitlin et al., 2013). It is therefore unlikely that 
A. niger spores become easily inactivated due to space radiation alone. We thus recommend that current planetary protection guidelines are revisited to address the high resistance of fungal spores in space travel scenarios. In addition, further studies are needed in order to address fungal spore resistance to other space environmental factors such as vacuum, changes in pressure, and extreme temperature fluctuations.

\section{DATA AVAILABILITY STATEMENT}

All datasets generated for this study are included in the article/Supplementary Material.

\section{AUTHOR CONTRIBUTIONS}

$\mathrm{MC}, \mathrm{AH}$, and $\mathrm{RU}$ performed the experiments, analyzed the data, and wrote the manuscript. VM, TS, RM, and AF contributed to the conception and design of the study, and manuscript preparation.

\section{REFERENCES}

Angelova, M. B., Pashova, S. B., Spasova, B. K., Vassilev, S. V., and Slokoska, L. S. (2005). Oxidative stress response of filamentous fungi induced by hydrogen peroxide and paraquat. Mycol. Res. 109, 150-158. doi: 10.1017/ s0953756204001352

Bauermeister, A., Bentchikou, E., Moeller, R., and Rettberg, P. (2009). Roles of PprA, IrrE, and RecA in the resistance of Deinococcus radiodurans to germicidal and environmentally relevant UV radiation. Arch. Microbiol. 191, 913-918. doi: 10.1007/s00203-009-0522-7

Beauvais, A., Fontaine, T., Aimanianda, V., and Latge, J. P. (2014). Aspergillus cell wall and biofilm. Mycopathologia 178, 371-377. doi: 10.1007/s11046-0149766-0

Blachowicz, A., Raffa, N., Bok, J. W., Choera, T., Knox, B., Lim, F. Y., et al (2020). Contributions of spore secondary metabolites to UV-C protection and virulence vary in different Aspergillus fumigatus Strains. mBio 11:e03415-19. doi: $10.1128 / \mathrm{mBio} .03415-19$

Bos, C. J., Debets, A. J., Swart, K., Huybers, A., Kobus, G., and Slakhorst, S. M. (1988). Genetic analysis and the construction of master strains for assignment of genes to six linkage groups in Aspergillus niger. Curr. Genet. 14, 437-443. doi: $10.1007 / \mathrm{bf} 00521266$

Cadet, J., Grand, A., and Douki, T. (2015). "Solar UV radiation-induced DNA bipyrimidine photoproducts: formation and mechanistic insights," in Photoinduced Phenomena in Nucleic Acids II: DNA Fragments and Phenomenological Aspects, eds M. Barbatti, A. C. Borin, and S. Ullrich (Cham: Springer International Publishing), 249-275. doi: 10.1007/128_ 2014_553

Cairns, T. C., Nai, C., and Meyer, V. (2018). How a fungus shapes biotechnology: 100 years of Aspergillus niger research. Fungal. Biol. Biotechnol. 5:13. doi: 10. 1186/s40694-018-0054-5

Carvalho, N. D., Arentshorst, M., Jin Kwon, M., Meyer, V., and Ram, A. F. (2010). Expanding the ku70 toolbox for filamentous fungi: establishment of complementation vectors and recipient strains for advanced gene analyses. Appl. Microbiol. Biotechnol. 87, 1463-1473. doi: 10.1007/s00253-0102588-1

Casadevall, A., Cordero, R. J. B., Bryan, R., Nosanchuk, J., and Dadachova, E. (2017). Melanin, radiation, and energy transduction in fungi. Microbiol Spectr 5, 1-6.

Chancellor, J. C., Blue, R. S., Cengel, K. A., Auñón-Chancellor, S. M., Rubins, K. H., Katzgraber, H. G., et al. (2018). Limitations in predicting the space radiation

\section{FUNDING}

RM was supported by the DLR grant FuE-Projekt "ISS LIFE" (Programm RF-FuW, Teilprogramm 475). MC was supported by the DLR/DAAD Research Fellowship Doctoral Studies in Germany, 2017 (57370122). AF and RM - MEXT Grant-in-Aid for Scientific Research on Innovative Areas "Living in Space" (Grant Numbers: 15H05935, 15K21745).

\section{ACKNOWLEDGMENTS}

We thank Andrea Schröder and the HIMAC operators for their excellent technical assistance during parts of this research.

\section{SUPPLEMENTARY MATERIAL}

The Supplementary Material for this article can be found online at: https://www.frontiersin.org/articles/10.3389/fmicb. 2020.00560/full\#supplementary-material

health risk for exploration astronauts. NPJ Micrograv. 4:8. doi: 10.1038/s41526 018-0043-2

Chancellor, J. C., Scott, G. B. I., and Sutton, J. P. (2014). Space radiation: the number one risk to astronaut health beyond low earth orbit. Life 4, 491-510. doi: 10.3390/life 4030491

Chang, P.-K., Cary, J. W., and Lebar, M. D. (2020). Biosynthesis of conidial and sclerotial pigments in Aspergillus species. Appl. Microbiol. Biotechnol. 104 2277-2286. doi: 10.1007/s00253-020-10347-y

Checinska, A., Probst, A. J., Vaishampayan, P., White, J. R., Kumar, D., Stepanov, V. G., et al. (2015). Microbiomes of the dust particles collected from the international space station and spacecraft assembly facilities. Microbiome 3:50. doi: $10.1186 /$ s40168-015-0116-3

Cockell, C. S., and Knowland, J. (1999). Ultraviolet radiation screening compounds. Biol. Rev. Camb. Philos. Soc. 74, 311-345. doi: 10.1111/j.1469-185x.1999. tb00189.x

Cordero, R. J., and Casadevall, A. (2017). Functions of fungal melanin beyond virulence. Fungal Biol. Rev. 31, 99-112. doi: 10.1016/j.fbr.2016.12.003

Cortesão, M., Schütze, T., Marx, R., Moeller, R., and Meyer, V. (2020). "Fungal biotechnology in space: why and how?" in Grand Challenges in Fungal Biotechnology, ed. H. Nevalainen (Cham: Springer International Publishing), 501-535. doi: 10.1007/978-3-030-29541-7_18

Cucinotta, F. A., and Durante, M. (2006). Cancer risk from exposure to galactic cosmic rays: implications for space exploration by human beings. Lancet. Oncol. 7,431-435. doi: 10.1016/s1470-2045(06)70695-7

Cucinotta, F. A., Kim, M.-H. Y., Chappell, L. J., and Huff, J. L. (2013). How safe is safe enough? Radiation risk for a human mission to Mars. PLoS One 8:e74988. doi: 10.1371/journal.pone.0074988

Dadachova, E., Bryan, R. A., Huang, X., Moadel, T., Schweitzer, A. D., et al. (2007). Ionizing radiation changes the electronic properties of melanin and enhances the growth of melanized fungi. PLoS One 2:e457. doi: 10.1371/journal.pone 0000457

Driedger, A. A., James, A. P., and Grayston, M. J. (1970). Cell survival and X-ray-induced DNA degradation in Micrococcus radiodurans. Radiat. Res. 44 835-845

Eckardt-Schupp, F., and Klaus, C. (1999). Radiation inducible DNA repair processes in eukaryotes. Biochimie 81, 161-171. doi: 10.1016/s0300-9084(99) 80049-2

Eisenman, H. C., and Casadevall, A. (2012). Synthesis and assembly of fungal melanin. Appl. Microbiol. Biotechnol. 93, 931-940. doi: 10.1007/s00253-011 $3777-2$ 
Esbelin, J., Mallea, S., Ram, A. F., and Carlin, F. (2013). Role of pigmentation in protecting Aspergillus niger conidiospores against pulsed light radiation. Photochem. Photobiol. 89, 758-761. doi: 10.1111/php.12037

Goins, C. L., Gerik, K. J., and Lodge, J. K. (2006). Improvements to gene deletion in the fungal pathogen Cryptococcus neoformans: absence of $\mathrm{Ku}$ proteins increases homologous recombination, and co-transformation of independent DNA molecules allows rapid complementation of deletion phenotypes. Fungal. Genet. Biol. 43, 531-544. doi: 10.1016/j.fgb.2006.02.007

Goldman, G. H., Mcguire, S. L., and Harris, S. D. (2002). The DNA damage response in filamentous fungi. Fungal Genet. Biol. 35, 183-195. doi: 10.1006/ fgbi.2002.1344

Horneck, G., Klaus, D. M., and Mancinelli, R. L. (2010). Space microbiology. Microbiol. Mol. Biol. Rev. 74, 121-156. doi: 10.1128/MMBR.000 16-09

Hutchinson, F., Preston, A., and Vogel, B. (1957). Radiation sensitivity of enzymes in wet and in dry yeast cells. Radiat. Res. 7, 465-472.

Ikpeme, S., Lobrich, M., Akpa, T., Schneider, E., and Kiefer, J. (1995). Heavy ion-induced DNA double-strand breaks with yeast as a model system. Radiat. Environ. Biophys. 34, 95-99. doi: 10.1007/bf01275213

Jorgensen, T. R., Park, J., Arentshorst, M., Van Welzen, A. M., Lamers, G., Vankuyk, P. A., et al. (2011). The molecular and genetic basis of conidial pigmentation in Aspergillus niger. Fungal Genet Biol. 48, 544-553. doi: 10.1016/j.fgb.2011. 01.005

Kennedy, A. R. (2014). Biological effects of space radiation and development of effective countermeasures. Life Sci. Space Res. 1, 10-43. doi: 10.1016/j.lssr.2014. 02.004

Khodadad, C. L., Wong, G. M., James, L. M., Thakrar, P. J., Lane, M. A., Catechis, J. A., et al. (2017). Stratosphere conditions inactivate bacterial endospores from a Mars spacecraft assembly facility. Astrobiology 17, 337-350. doi: 10.1089/ast. 2016.1549

Kiefer, J., Egenolf, R., and Ikpeme, S. (2002). Heavy ion-induced DNA doublestrand breaks in yeast. Radiat. Res. 157, 141-148. doi: 10.1667/0033-7587(2002 157[0141:hiidds]2.0.co;2

Klintworth, R., and Reher, H. J. (1999). Biological induced corrosion of material II: new test methods and experiences from mir station. Acta Astronaut. 44, 569-578. doi: 10.1016/s0094-5765(99)00069-7

Kminek, G., Fellous, J., Rettberg, P., Moissl-Eichinger, C., Sephton, M. A., Royle S. H., et al. (2019). The international planetary protection handbook. Space Res Today 205, e1-e120. doi: 10.1016/j.srt.2019.09.001

Krijgsheld, P., Bleichrodt, R., Van Veluw, G. J., Wang, F., Müller, W. H. Dijksterhuis, J., et al. (2013). Development in Aspergillus. Stud. Mycol. 74, 1-29. doi: $10.3114 /$ sim0006

Kwon, M. J., Arentshorst, M., Roos, E. D., Van Den Hondel, C. A., Meyer, V., and Ram, A. F. (2011). Functional characterization of Rho GTPases in Aspergillus niger uncovers conserved and diverged roles of Rho proteins within filamentous fungi. Mol. Microbiol. 79, 1151-1167. doi: 10.1111/j.1365-2958.2010.07524.x

Kwon, M. J., Nitsche, B. M., Arentshorst, M., Jorgensen, T. R., Ram, A. F. and Meyer, V. (2013). The transcriptomic signature of RacA activation and inactivation provides new insights into the morphogenetic network of Aspergillus niger. PLoS One 8:e68946. doi: 10.1371/journal.pone.0068946

Latgé, J. P. (1999). Aspergillus fumigatus and aspergillosis. Clin. Microbiol. Rev. 12 310-350. doi: 10.1128/CMR.12.2.310

Ma, L., Kazama, Y., Inoue, H., Abe, T., Hatakeyama, S., and Tanaka, S. (2013). The type of mutations induced by carbon-ion-beam irradiation of the filamentous fungus Neurospora crassa. Fungal. Biol. Biotechnol. 117, 227-238. doi: 10.1016/ j.funbio.2013.01.002

Makimura, K., Hanazawa, R., Takatorl, K., Tamura, Y., Fujisaki, R., Nishiyama, Y., et al. (2001). Fungal flora on board the Mir-space station, identification by morphological features and ribosomal DNA sequences. Microbiol. Immunol. 45 , 357-363. doi: 10.1111/j.1348-0421.2001.tb02631.x

McFadden, D. C., and Casadevall, A. (2001). Capsule and melanin synthesis in Cryptococcus neoformans. Med. Mycol. 39(Suppl. 1), 19-30. doi: 10.1080/ 744118883

Meechan, P. J., and Wilson, C. (2006). Use of ultraviolet lights in biological safety cabinets: a contrarian view. Appl. Biosaf. 11, 222-227. doi: 10.1177/ 153567600601100412

Meyer, V., Arentshorst, M., El-Ghezal, A., Drews, A. C., Kooistra, R., Van Den Hondel, C. A., et al. (2007). Highly efficient gene targeting in the Aspergillus niger kusA mutant. J. Biotechnol. 128, 770-775. doi: 10.1016/j.jbiotec.2006. 12.021

Meyer, V., Fiedler, M., Nitsche, B., and King, R. (2015)، “The cell factory Aspergillus enters the big data era: opportunities and challenges for optimising product formation," in Filaments in Bioprocesses, eds R. Krull and T. Bley (Cham Springer), 91-132. doi: 10.1007/10_2014_297

Moeller, R., Raguse, M., Leuko, S., Berger, T., Hellweg, C. E., Fujimori, A., et al. (2017). STARLIFE-An international campaign to study the role of galactic cosmic radiation in astrobiological model systems. Astrobiology 17, 101-109. doi: 10.1089 /ast.2016.1571

Moeller, R., Raguse, M., Reitz, G., Okayasu, R., Li, Z., Klein, S., et al. (2014). Resistance of Bacillus subtilis spore DNA to lethal ionizing radiation damage relies primarily on spore core components and DNA repair, with minor effects of oxygen radical detoxification. Appl. Environ. Microbiol. 80, 104-109. doi: 10.1128/AEM.03136-13

Moeller, R., Reitz, G., Berger, T., Okayasu, R., Nicholson, W. L., and Horneck, G. (2010). Astrobiological aspects of the mutagenesis of cosmic radiation on bacterial spores. Astrobiology 10, 509-521. doi: 10.1089/ast.2009.0429

Mora, M., Mahnert, A., Koskinen, K., Pausan, M. R., Oberauner-Wappis, L., Krause, R., et al. (2016). Microorganisms in confined habitats: microbia monitoring and control of intensive care units, operating rooms, cleanrooms and the international space station. Front. Microbiol. 7:1573. doi: 10.3389/fmicb. 2016.01573

Mora, M., Wink, L., Kögler, I., Mahnert, A., Rettberg, P., Schwendner, P., et al. (2019). Space Station conditions are selective but do not alter microbial characteristics relevant to human health. Nat. Commun. 10:3990.

Moseley, B. E., and Laser, H. (1965). Repair of X-ray in Micrococcus radiodurans. Proc. R. Soc. Lond. B. Biol. Sci. 162, 210-222. doi: 10.1098/rspb.1965.0035

Mowat, E., Butcher, J., Lang, S., Williams, C., and Ramage, G. (2007). Development of a simple model for studying the effects of antifungal agents on multicellular communities of Aspergillus fumigatus. J. Med. Microbiol. 56, 1205-1212. doi: 10.1099/jmm.0.47247-0

Narici, L., Casolino, M., Di Fino, L., Larosa, M., Picozza, P., Rizzo, A., et al. (2017). Performances of Kevlar and Polyethylene as radiation shielding on-board the International Space Station in high latitude radiation environment. Sci. Rep. 7 1644-1644. doi: 10.1038/s41598-017-01707-2

Newcombe, D. A., Schuerger, A. C., Benardini, J. N., Dickinson, D., Tanner, R., and Venkateswaran, K. (2005). Survival of spacecraft-associated microorganisms under simulated martian UV irradiation. Appl. Environ. Microbiol. 71, 81478156. doi: $10.1128 /$ aem.71.12.8147-8156.2005

Ninomiya, Y., Suzuki, K., Ishii, C., and Inoue, H. (2004). Highly efficient gene replacements in Neurospora strains deficient for nonhomologous end-joining. Proc. Natl. Acad. Sci. U.S.A. 101:12248. doi: 10.1073/pnas.0402780101

Novikova, N., De Boever, P., Poddubko, S., Deshevaya, E., Polikarpov, N., Rakova, N., et al. (2006). Survey of environmental biocontamination on board the International Space Station. Res. Microbiol. 157, 5-12. doi: 10.1016/j.resmic. 2005.07.010

Pacelli, C., Bryan, R. A., Onofri, S., Selbmann, L., Shuryak, I., and Dadachova, E. (2017). Melanin is effective in protecting fast and slow growing fungi from various types of ionizing radiation. Environ. Microbiol. 19, 1612-1624. doi $10.1111 / 1462-2920.13681$

Riesenman, P. J., and Nicholson, W. L. (2000). Role of the spore coat layers in Bacillus subtilis spore resistance to hydrogen peroxide, artificial UV-C. UV-B, and solar UV radiation. Appl. Environ. Microbiol. 66, 620-626. doi: 10.1128/ aem.66.2.620-626.2000

Roper, M., Ellison, C., Taylor, J. W., and Glass, N. L. (2011). Nuclear and genome dynamics in multinucleate ascomycete fungi. Curr. Biol. 21, R786-R793. doi: 10.1016/j.cub.2011.06.042

Segers, F. J. J., Wosten, H. A. B., and Dijksterhuis, J. (2018). Aspergillus niger mutants affected in conidial pigmentation do not have an increased susceptibility to water stress during growth at low water activity. Lett. Appl. Microbiol. 66, 238-243. doi: 10.1111/lam.12846

Setlow, P. (2014). Spore resistance properties. Microbiol. Spectr. 2, 1-14.

Silverman, G. J., Davis, N. S., and Beecher, N. (1967). Resistivity of spores to ultraviolet and gamma radiation while exposed to ultrahigh vacuum or at atmospheric pressure. Appl. Microbiol. 15, 510-515. doi: 10.1128/aem.15.3.510 515.1967 
Singaravelan, N., Grishkan, I., Beharav, A., Wakamatsu, K., Ito, S., and Nevo, F. (2008). Adaptive melanin response of the soil fungus Aspergillus niger to UV radiation stress at "evolution canyon", mount carmel, Israel. PLoS One 3:e2993. doi: 10.1371/journal.pone.0002993

Sinha, R. P., and Hader, D. P. (2002). A UV-induced DNA damage and repair: review. Photochem. Photobiol. Sci. 1, 225-236. doi: 10.1039/b201230h

Sisti, M., Schiavano, G. F., Santi, M. D., and Brandi, G. (2017). Ultraviolet germicidal irradiation in tap water contaminated by Aspergillus spp. J. Prev. Med. Hyg. 58, E315-E319. doi: 10.15167/2421-4248/jpmh2017.58.4.777

Sliney, D. H. (2007). Radiometric quantities and units used in photobiology and photochemistry: recommendations of the commission internationale de l'eclairage (international commission on illumination). Photochem. Photobiol. 83, 425-432. doi: 10.1562/2006-11-14-ra-1081

Yang, J. H., Wu, U. I., Tai, H. M., and Sheng, W. H. (2019). Effectiveness of an ultraviolet-C disinfection system for reduction of healthcare-associated pathogens. J. Microbiol. Immunol. Infect. 52, 487-493. doi: 10.1016/j.jmii.2017. 08.017

Zeitlin, C., Hassler, D. M., Cucinotta, F. A., Ehresmann, B., WimmerSchweingruber, R. F., Brinza, D. E., et al. (2013). Measurements of energetic particle radiation in transit to mars on the mars science laboratory. Science 340, 1080-1084. doi: 10.1126/science.123 5989

Zhdanova, N. N., Zakharchenko, V. A., Vember, V. V., and Nakonechnaya, L. T. (2000). Fungi from chernobyl: mycobiota of the inner regions of the containment structures of the damaged nuclear reactor. Mycol. Res. 104, 14211426. doi: $10.1017 / \mathrm{s} 0953756200002756$

Conflict of Interest: The authors declare that the research was conducted in the absence of any commercial or financial relationships that could be construed as a potential conflict of interest.

Copyright (๑) 2020 Cortesão, de Haas, Unterbusch, Fujimori, Schütze, Meyer and Moeller. This is an open-access article distributed under the terms of the Creative Commons Attribution License (CC BY). The use, distribution or reproduction in other forums is permitted, provided the original author(s) and the copyright owner(s) are credited and that the original publication in this journal is cited, in accordance with accepted academic practice. No use, distribution or reproduction is permitted which does not comply with these terms. 


\section{frontiers}

\section{Supplementary Material}

\section{Supplementary Figures}

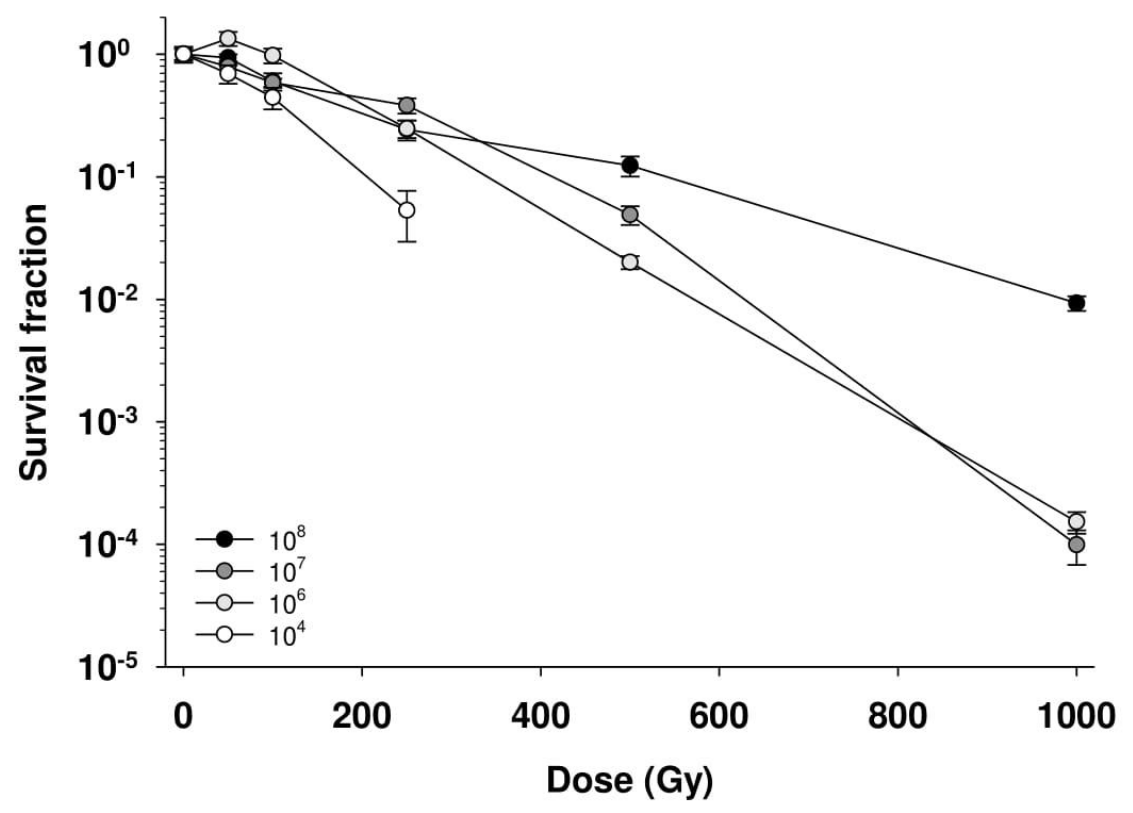

Supplementary Figure 1. Effect of initial spore concentration (inoculum) in survivability towards ionizing (X-ray) radiation. At $10^{8}$ spores $/ \mathrm{ml}$ there is a spore multi-layer that can provide radiation shielding, whereas at $10^{6}$ and $10^{7}$ spores $/ \mathrm{ml}$ there is a spore monolayer, preventing self-shielding from the spores. No CFU were detected after $250 \mathrm{~Gy}$ for $10^{4}$ spores $/ \mathrm{ml}$. 


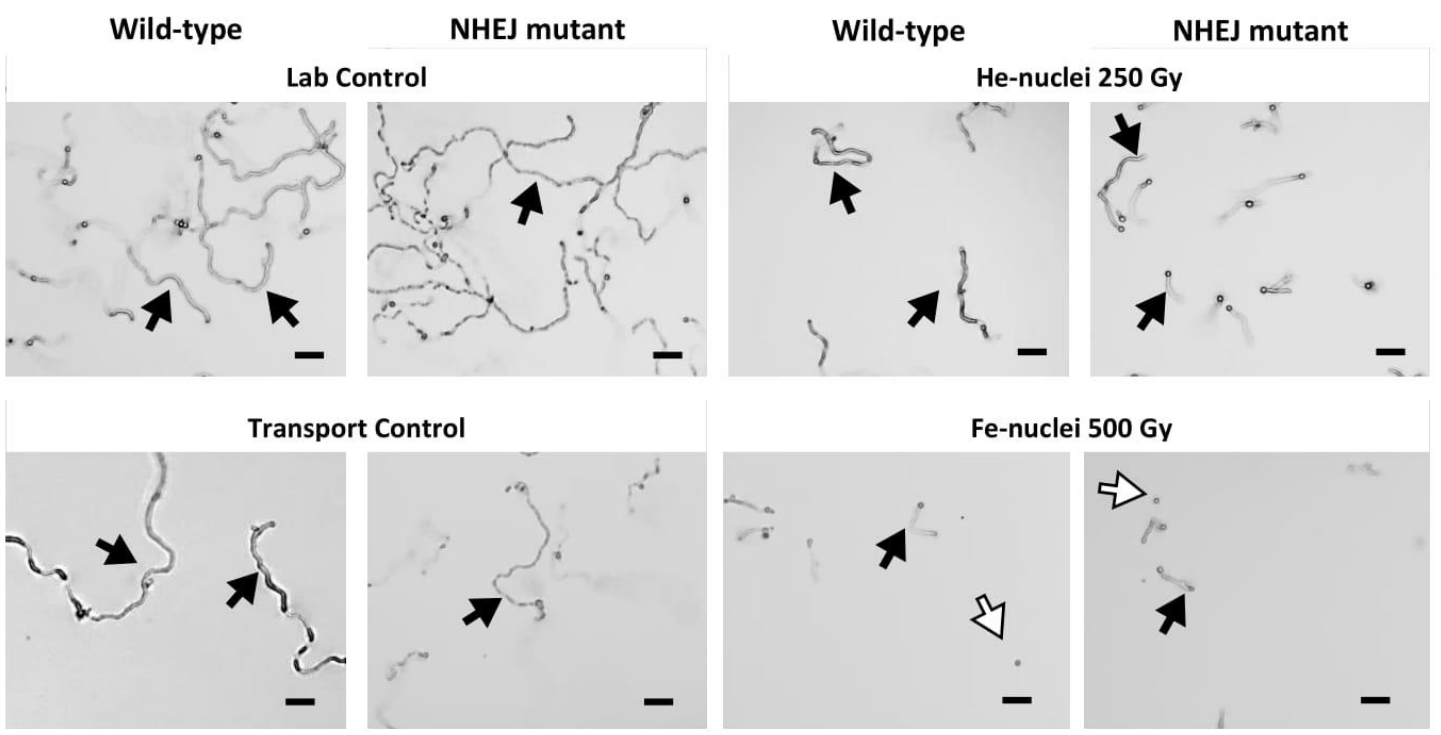

Supplementary Figure 2. Germination of A. niger spores after He- and Fe-nuclei irradiation. Black arrows indicate regions of interest showing spore germination and varying hyphal length as the radiation dose increases. At $500 \mathrm{~Gy}$ of Fe-nuclei radiation there $\mathrm{CFU}$ were detected, but spore germination can still be identified. White arrows indicate un-germinated spores. Pictures were taken with after one day of incubation on MM-agar plates with an Axio Imager. M2 at $100 \mathrm{x}$ magnification. Each picture was chosen as representative for the respective condition. Scale bar: $50 \mu \mathrm{m}$. 


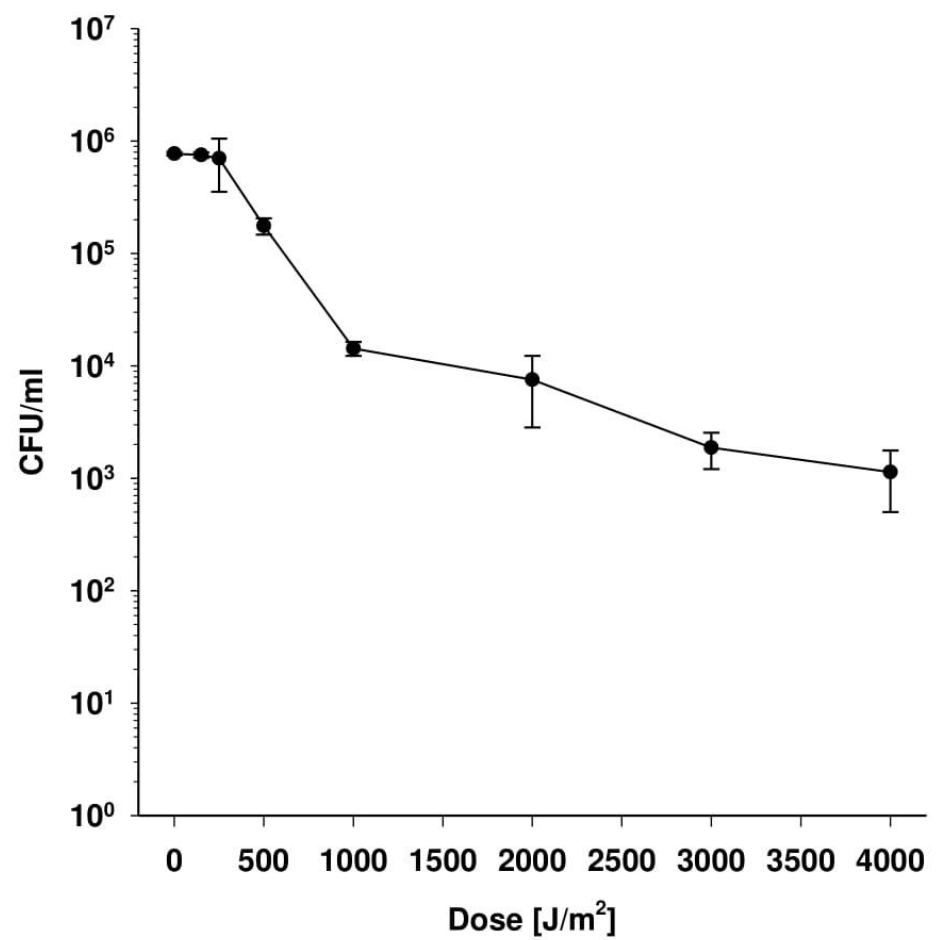

Supplementary Figure 3. UV-C survival curve of Aspergillus niger wild-type spores showing dose-effect. Spores irradiated in $0.9 \% \mathrm{NaCl}\left(10^{6}\right.$ spores $\left./ \mathrm{ml}\right)$, in air. Survivability was calculated as colony-forming units (CFU) per $\mathrm{ml}^{-\mathrm{LD}_{90}}$ value is $1038 \mathrm{~J} /{ }^{\mathrm{m} 2}$. 


\section{Chapter II: MARSBOx: Bacterial and fungal endurance from a balloon-flown analog mission in the stratosphere}

\section{Publication}

Cortesão, M. and Siems, K., Koch, S., Beblo-Vranesevic, K., Rabbow, E., Berger, T., Lane, M., James, L., Johnson, P., Waters, S.M, Verma, S.D., Smith, D.J., and Moeller, R (2021). MARSBOx: Fungal and bacterial endurance from a balloon-flown analog mission in the stratosphere. Frontiers in Microbiology. 12:601713.

\section{Author contribution}

Planning: $30 \%$, Experiment: $50 \%$, Manuscript: $50 \%$

$M C, K S, S K$, and KB-V performed the microbial experiments, analyzed the data, and wrote the manuscript. DS, SW, SV, ER, and RM contributed to the conception and design of the study, data analyses and manuscript preparation. DS, ML, LJ, and PJ prepared and performed the balloon flight mission and contributed to manuscript preparation. TB contributed with the M-42 experiment and manuscript preparation. All authors contributed to the article and approved the submitted version. 
OPEN ACCESS

Edited by:

Jesse G. Dillon,

California State University, Long Beach, United States

Reviewed by:

Claudia Coleine,

University of Tuscia, Italy

Kai Waldemar Finster,

Aarhus University, Denmark

Anima Nanda,

Sathyabama Institute of Science

and Technology, India

*Correspondence:

Ralf Moeller

ralf.moeller@dlr.de

David J. Smith

david.j.smith-3@nasa.gov

${ }^{\dagger}$ These authors have contributed equally to this work and share first

authorship

Specialty section: This article was submitted to

Extreme Microbiology,

a section of the journal

Frontiers in Microbiology

Received: 01 September 2020

Accepted: 20 January 2021

Published: 22 February 2021

Citation:

Cortesão M, Siems K, Koch S, Beblo-Vranesevic K, Rabbow E,

Berger T, Lane $M$, James $L$, Johnson $P$, Waters SM, Verma SD,

Smith DJ and Moeller R (2021) MARSBOX: Fungal and Bacterial Endurance From a Balloon-Flown Analog Mission in the Stratosphere.

Front. Microbiol. 12:601713. doi: 10.3389/fmicb.2021.601713

\section{MARSBOx: Fungal and Bacterial Endurance From a Balloon-Flown Analog Mission in the Stratosphere}

\author{
Marta Cortesão ${ }^{1 \dagger}$, Katharina Siems ${ }^{1 \dagger}$, Stella Koch ${ }^{1}$, Kristina Beblo-Vranesevic ${ }^{2}$, \\ Elke Rabbow ${ }^{2}$, Thomas Berger ${ }^{3}$, Michael Lane ${ }^{4}$, Leandro James ${ }^{4}$, Prital Johnson ${ }^{4}$, \\ Samantha M. Waters ${ }^{5,6}$, Sonali D. Verma ${ }^{6,7}$, David J. Smith ${ }^{6 *}$ and Ralf Moeller ${ }^{1 *}$
}

'Aerospace Microbiology Research Group, Department of Radiation Biology, Institute of Aerospace Medicine, German Aerospace Center, Cologne, Germany, ${ }^{2}$ Astrobiology Research Group, Department of Radiation Biology, Institute of Aerospace Medicine, German Aerospace Center, Cologne, Germany, ${ }^{3}$ Biophysics Research Group, Department of Radiation Biology, Institute of Aerospace Medicine, German Aerospace Center, Cologne, Germany, ${ }^{4}$ NASA Kennedy Space Center, Engineering Directorate, Kennedy Space Center, Merritt Island, FL, United States, ${ }^{5}$ Universities Space Research Association, Moffett Field, CA, United States, ${ }^{6}$ NASA Ames Research Center, Space Biosciences Research Branch, Moffett Field, CA, United States, ${ }^{7}$ Blue Marble Space Institute of Science, Moffett Field, CA, United States

Whether terrestrial life can withstand the martian environment is of paramount interest for planetary protection measures and space exploration. To understand microbial survival potential in Mars-like conditions, several fungal and bacterial samples were launched in September 2019 on a large NASA scientific balloon flight to the middle stratosphere ( $\sim 38 \mathrm{~km}$ altitude) where radiation levels resembled values at the equatorial Mars surface. Fungal spores of Aspergillus niger and bacterial cells of Salinisphaera shabanensis, Staphylococcus capitis subsp. capitis, and Buttiauxella sp. MASE-IM-9 were launched inside the MARSBOx (Microbes in Atmosphere for Radiation, Survival, and Biological Outcomes Experiment) payload filled with an artificial martian atmosphere and pressure throughout the mission profile. The dried microorganisms were either exposed to full UV-VIS radiation (UV dose $=1148 \mathrm{~kJ} \mathrm{~m}^{-2}$ ) or were shielded from radiation. After the 5-h stratospheric exposure, samples were assayed for survival and metabolic changes. Spores from the fungus $A$. niger and cells from the Gram-(-) bacterium S. shabanensis were the most resistant with a 2- and 4-log reduction, respectively. Exposed Buttiauxella sp. MASE-IM-9 was completely inactivated (both with and without UV exposure) and S. capitis subsp. capitis only survived the UV shielded experimental condition (3-log reduction). Our results underscore a wide variation in survival phenotypes of spacecraft associated microorganisms and support the hypothesis that pigmented fungi may be resistant to the martian surface if inadvertently delivered by spacecraft missions.

Keywords: Mars simulation, fungal spores, spore survival, space, radiation, UV, balloon flight, stress resistance

\section{INTRODUCTION}

Mariner IV was the first successful robotic mission to Mars producing surface photos and preliminary data used to model atmospheric pressure, layer heights, and temperature (Leighton et al., 1965; Binder, 1966; Fjeldbo and Eshleman, 1968). In the half century since that first pioneering mission, orbital and surface rover missions have continued to characterize the Mars 
environment - generally regarded as hostile to terrestrial life as we know it. The martian surface features highly desiccating conditions as well as extremely low pressure and temperature. Moreover, lacking a substantial atmosphere and with a weak magnetosphere (Acuña et al., 2001), non-ionizing UV radiation $(100-400 \mathrm{~nm})$ as well as high energy solar ionizing radiation (Xrays, Gamma rays, etc.) and galactic cosmic rays (GCR) bombard the planet's surface (Kuhn and Atreya, 1979; Simonsen et al., 1990; Saganti et al., 2004; Hellweg and Baumstark-Khan, 2007; Barlow, 2008; Catling, 2009; Hassler et al., 2014; Martínez et al., 2017). With such an extreme radiation environment, from a terrestrial standpoint, the martian surface appears to be biocidal.

To ascertain where life can survive beyond Earth, experiments in the fields of space biology and astrobiology have examined the responses of terrestrial model organisms to simulated and real space conditions (Rothschild and Mancinelli, 2001; MoisslEichinger et al., 2016; DasSarma et al., 2020). Understanding microbial adaptations to either isolated and combined extreme environmental stressors helps (i) establish the limits of life on Earth as we know it; (ii) determine whether terrestrial life could survive on Mars; and (iii) refine the search for life in other extraterrestrial habitats (Horneck et al., 2010; Cockell et al., 2016). With the possible exception of the Viking missions, Mars has been unavailable to date for conducting controlled biological experiments; thus, extreme terrestrial analog environments have been widely used to test instrumentation and microbial survival outcomes (Marlow et al., 2008; Fairén et al., 2010; Suedfeld, 2010; West et al., 2010). Typically, martian analog environments are located on Earth's surface in regions where aridity, temperature extremes, and elevated radiation dominate the landscape. For instance, the McMurdo Dry Valleys in continental Antarctica and high-elevation deserts in Australia and South America are frequently visited, analog destinations (Clarke and Persaud, 2004; Fletcher et al., 2012; López-Lozano et al., 2012; Heldmann et al., 2013; Musilova et al., 2015). However, high above Earth's surface in the stratosphere $(\sim 15-50 \mathrm{~km})$, another Mars analog environment exists, presenting a unique combination of environmental insults that more closely resemble conditions on the Red Planet. In the middle stratosphere during daytime hours, the following Mars-like factors are simultaneously present: intense, full spectrum ultraviolet (UV) radiation, high energy ionizing radiation (including secondary scattering), desiccation, hypoxia, and ultralow temperatures and pressures (Clark and McCoy, 1965; Potemra and Zmuda, 1970; Vampola and Gorney, 1983; Keating et al., 1987; Clancy and Muhleman, 1993; Von Engeln et al., 1998; Seele and Hartogh, 1999; Shepherd, 2000; Lambert et al., 2007; Mertens et al., 2016; Caro et al., 2019). Taken together, these combined conditions cannot be found naturally anywhere on the surface of the Earth and would be challenging to easily reproduce in laboratory-based experiments.

Reaching the middle stratosphere is relatively achievable compared to suborbital and orbital spaceflight investigations. High-altitude scientific balloons have been used for more than eight decades to study the atmosphere and atmospheric phenomena (Winckler et al., 1959; Winckler, 1960; Murcray et al., 1969; Mertens et al., 2016; Caro et al., 2019) and more recently for conducting biological exposure experiments
(Stevens, 1936; Simons, 1954; Sullivan and Smith, 1960; Rainwater and Smith, 2004; Beck-Winchatz and Bramble, 2014; Coleman and Mitchell, 2014; Smith et al., 2014; Khodadad et al., 2017; Smith and Sowa, 2017; Pulschen et al., 2018). In this study, we take advantage of a large scientific balloon mission to the middle stratosphere ( $\sim 38 \mathrm{~km}$ altitude) for exposing microorganisms and measuring their survival and metabolic responses while monitoring ionizing radiation levels and other pertinent environmental conditions. Four microorganisms relevant to astrobiology and space biology were flown inside the Microbes in Atmosphere for Radiation, Survival, and Biological Outcomes Experiment (MARSBOx) payload. The two bacterial extremophiles, Salinisphaera shabanensis and Buttiauxella sp. MASE-IM-9, were included to test the hypothesis that terrestrial microbial strains, isolated from extreme Marsanalog environments, can withstand the stress factors of a martian-like environment. The fungus Aspergillus niger and the bacterium Staphylococcus capitis subsp. capitis were included in this study because they are human-associated and opportunistic pathogens, and have both been previously detected on the International Space Station (ISS). Thus, they are likely to travel to Mars in crewed space missions (Novikova et al., 2006; Checinska et al., 2015; Be et al., 2017; Mora et al., 2019; Sobisch et al., 2019). Moreover, spores from A. niger might resist space travel on the outside of a spacecraft; therefore, understanding their survival potential in a Mars-like environment is of interest to planetary protection.

The MARSBOx design was a balloon-compatible, NASAadapted version of hardware from the European Space Agency's (ESA) biological exposure missions EXPOSE-E and EXPOSE$\mathrm{R}$ aboard the ISS (Rabbow et al., 2009; Rabbow et al., 2012, 2015), using the transport and exposure box (Trex-Box) from the European MASE project (Beblo-Vranesevic et al., 2017a). To adjust for Mars atmospheric conditions, the Trex-Box was filled with a Mars gas mixture at 5-10 mbar during the mission. Herein, we report results from the first MARSBOx mission and summarize environmental conditions that collectively indicate the most robust Mars analog.

\section{MATERIALS AND METHODS}

\section{Test Organisms, Media, and Sample Preparation for Flight}

A summary of the microorganisms used in this study can be found in Table 1. Aspergillus niger (N402) spores were harvested after 3 days of incubation at $30^{\circ} \mathrm{C}$ from complete medium agar plates [CM; composition: $55 \mathrm{mM}$ glucose, $11 \mathrm{mM} \mathrm{KH}_{2} \mathrm{PO}_{4}$, $7 \mathrm{mM} \mathrm{KCl}, 178 \mathrm{nM} \mathrm{H}_{3} \mathrm{BO}_{3}, 2 \mathrm{mM} \mathrm{MgSO}_{4}, 76 \mathrm{nM} \mathrm{ZnSO}_{4}, 70 \mathrm{mM}$ $\mathrm{NaNO}_{3}, 6.2 \mathrm{nM} \mathrm{Na}_{2} \mathrm{MoO}_{4}, 18 \mathrm{nM} \mathrm{FeSO}_{4}, 7.1 \mathrm{nM} \mathrm{CoCl}_{2}, 6.4 \mathrm{nM}$ $\mathrm{CuSO}_{4}, 25 \mathrm{nM} \mathrm{MnCl}_{2}, 174 \mathrm{nM}$ EDTA; $0.5 \%\left(\mathrm{w} \mathrm{v}^{-1}\right)$ yeast extract and $0.1 \%\left(\mathrm{w} \mathrm{v}^{-1}\right)$ casamino acids, $15 \mathrm{~g}$ agar per Liter] by flooding the plates with sterile, saline solution $(0.9 \% \mathrm{NaCl})$ and gently scraping the spores out using a cotton stick.

The spore suspensions were then filtered through a sterile filter with 22-25 $\mu \mathrm{m}$ pore size (Miracloth) to remove hyphal fragments and kept at $4^{\circ} \mathrm{C}$. Titer determination was done using an improved 
TABLE 1 | Test microorganisms used in this study.

\begin{tabular}{|c|c|c|c|c|}
\hline Organism & Classification & Growth & Tested as & References \\
\hline Aspergillus niger $\mathrm{N} 402$ & $\begin{array}{l}\text { Filamentous fungus } \\
\text { (mold) }\end{array}$ & $\begin{array}{l}\text { Minimal Medium } \\
30^{\circ} \mathrm{C}\end{array}$ & $\begin{array}{l}\text { Spore monolayer }\left(10^{7} \text { spores }\right. \\
\left.\mathrm{mL}^{-1}\right) \text { or spore multilayer } \\
\left(10^{8} \text { spores } \mathrm{mL}^{-1}\right) \text { desiccated in } \\
\text { water }\end{array}$ & Bos et al., 1988 \\
\hline $\begin{array}{l}\text { Staphylococus capitis } \\
\text { subsp. capitis } \\
\mathrm{K} 1-2-2-23\end{array}$ & Gram-(+) bacteria & $\begin{array}{l}\text { Tryptic Soy Broth (TSB) or Agar } \\
\text { (TSA) } 37^{\circ} \mathrm{C}\end{array}$ & 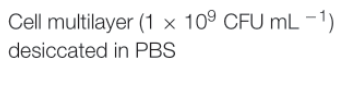 & Sobisch et al., 2019 \\
\hline $\begin{array}{l}\text { Salinisphaera } \\
\text { shabanensis }\end{array}$ & $\begin{array}{l}\text { Gram-(-) bacteria } \\
\text { Halophilic }\end{array}$ & Marine broth or agar $30^{\circ} \mathrm{C}$ & $\begin{array}{l}\text { Cell multilayer }\left(2 \times 10^{8} \mathrm{CFU} \mathrm{mL}^{-1}\right) \\
\text { desiccated in PBS }(10 \% \mathrm{NaCl}) \text { or } \\
\text { medium. }\end{array}$ & Antunes et al., 2003 \\
\hline $\begin{array}{l}\text { Buttiauxella sp. } \\
\text { MASE-IM-9 }\end{array}$ & Gram-(-) bacteria & $\begin{array}{l}\text { Tryptic Soy Broth (TSB) or Agar } \\
\text { (TSA) } 30^{\circ} \mathrm{C}\end{array}$ & $\begin{array}{l}\text { Cell multilayer }\left(2 \times 10^{8} \mathrm{CFU} \mathrm{mL}^{-1}\right) \\
\text { desiccated in PBS or medium. }\end{array}$ & Cockell et al., 2018 \\
\hline
\end{tabular}

Neubauer cell count chamber on vortexed suspensions; $20 \mu \mathrm{L}$ of spores were then spotted onto round quartz disks $(6 \mathrm{~mm} \varnothing$, $1 \mathrm{~mm}$ thickness; MolTech) in triplicate and left to dry at room temperature $\left(22^{\circ} \mathrm{C}\right)$ on the bench. Two spore concentrations were prepared for the MARSBOx flight: $1 \times 10^{7}$ spores $\mathrm{mL}^{-1}$ (spore monolayer) and $1 \times 10^{8}$ spores $\mathrm{mL}^{-1}$ (spore multilayer). Presence of spore multilayer and monolayer was determined qualitatively with a scanning electron microscope (JSM-6510, Jeol), operated at $10 \mathrm{Kv}$ (Figure 1). Further experiments using agar plates were done with minimal medium (composition: $55 \mathrm{mM}$ glucose, $11 \mathrm{mM} \mathrm{KH} 2 \mathrm{PO}_{4}, 7 \mathrm{mM} \mathrm{KCl}, 178 \mathrm{nM} \mathrm{H}_{3} \mathrm{BO}_{3}$, $2 \mathrm{mM} \mathrm{MgSO}_{4}, 76 \mathrm{nM} \mathrm{ZnSO}_{4}, 70 \mathrm{mM} \mathrm{NaNO}_{3}, 6.2 \mathrm{nM} \mathrm{Na}_{2} \mathrm{MoO} 4$, $18 \mathrm{nM} \mathrm{FeSO}_{4}, 7.1 \mathrm{nM} \mathrm{CoCl} 2,6.4 \mathrm{nM} \mathrm{CuSO}$, $25 \mathrm{nM} \mathrm{MnCl}_{2}$, 174 nM EDTA, 15 g agar, per Liter).

Staphylococcus capitis subsp. capitis strain K1-2-2-23 (DSM 111179) was cultivated in tryptic soy broth (TSB; BD Difco; composition: tryptone $17.0 \mathrm{~g}$, soy peptone $3.0 \mathrm{~g}$, glucose $2.5 \mathrm{~g}$, $\mathrm{NaCl} 5.0$ g, $\mathrm{K}_{2} \mathrm{HPO}_{4} 2.5 \mathrm{~g}$, per Liter) at $37^{\circ} \mathrm{C}$ under constant agitation at $200 \mathrm{rpm}$ for $18 \mathrm{~h}$. Stationary phase cells were harvested by centrifugation $(4000 \mathrm{rpm})$ in $40 \mathrm{~mL}$ culture for $5 \mathrm{~min}$. Cells were washed by removal of the supernatant, resuspension of the pellet in $40 \mathrm{~mL}$ phosphate buffered saline (PBS; composition: $\mathrm{Na}_{2} \mathrm{HPO}_{4} 7.0 \mathrm{~g}, \mathrm{KH}_{2} \mathrm{PO}_{4} 3.0 \mathrm{~g}, \mathrm{NaCl} 4.0 \mathrm{~g}$, per Liter, $\mathrm{pH}$ 7.5), and centrifugation for $5 \mathrm{~min}$ at $4000 \mathrm{rpm}$. The washing step was repeated once and after the last centrifugation step, the pellet was resuspended in $10 \mathrm{~mL}$ PBS. Thirty $\mu \mathrm{L}$ of this concentrated cell suspension were pipetted onto the quartz disks and left to dry at room temperature $\left(22^{\circ} \mathrm{C}\right)$ on the bench. The absolute number of cells per quartz disk was determined by standard plate counts on tryptic soy agar (TSA; composition: tryptone $17.0 \mathrm{~g}$, soy peptone $3.0 \mathrm{~g}$, glucose $2.5 \mathrm{~g}, \mathrm{NaCl} 5.0 \mathrm{~g}$, $\mathrm{K}_{2} \mathrm{HPO}_{4} 2.5 \mathrm{~g}$, agar $15 \mathrm{~g}$, per Liter) to be $1.4 \times 10^{9}$ cells per disk, resulting in a multilayer of bacterial cells.

The facultative anaerobes, Salinisphaera shabanensis and Buttiauxella sp. MASE-IM-9, were cultivated in liquid microoxic Marine Broth (BD Difco; composition: peptone 5.0 g, yeast extract $1.0 \mathrm{~g}$ ferric citrate $0.1 \mathrm{~g}, \mathrm{NaCl} 19.45 \mathrm{~g}, \mathrm{MgCl}_{2} 5.9 \mathrm{~g}$, $\mathrm{MgSO}_{4} 3.24 \mathrm{~g}, \mathrm{CaCl}_{2} 1.8 \mathrm{~g}, \mathrm{KCl} 0.55 \mathrm{~g}, \mathrm{NaHCO}_{3} 0.16 \mathrm{~g}, \mathrm{KBr}$, $0.08 \mathrm{~g}, \mathrm{SrCl}_{2} 34.0 \mathrm{mg}$, boric acid $22.0 \mathrm{mg}, \mathrm{Na}_{4} \mathrm{SiO}_{4} 4.0 \mathrm{mg}$, $\mathrm{NaF} 2.4 \mathrm{mg}, \mathrm{NH}_{4} \mathrm{NO}_{3} 1.6 \mathrm{mg}$, and $\mathrm{Na}_{2} \mathrm{HPO}_{4} 8.0 \mathrm{mg}$, per Liter) and microoxic TSB, respectively, in sterile serum bottles with headspace pressure and gas composition of $\sim 1,010$ mbar and $\mathrm{N}_{2} / \mathrm{CO}_{2}(80 / 20 \mathrm{v} / \mathrm{v})$, respectively. Cell concentration was determined via cell counting in a Thoma counting chamber. Stationary phase cells from an overnight culture of each strain were harvested by centrifugation $(14,500 \mathrm{~g})$ of $1 \mathrm{~mL}$ culture for $15 \mathrm{~min}$. To assess possible differences in survival between cells dried in medium (UV absorption of medium components) or dried in non-absorbing buffer, after the centrifugation step either (i) $950 \mu \mathrm{L}$ of the supernatant were removed, cells were resuspended in the remaining growth media, and $50 \mu \mathrm{L}$ were applied on each quartz disk; or (ii) cells were washed with $1 \mathrm{~mL}$ PBS (for S. shabanensis the $\mathrm{NaCl}$ content was adjusted to $10 \%$ ), again centrifuged for $15 \mathrm{~min}$ at $14,100 \mathrm{~g}$, the supernatant $(950 \mu \mathrm{L})$ was removed, and $50 \mu \mathrm{L}$ were applied on each quartz disk. The desiccation process was conducted under oxic conditions on the bench. The absolute number of cells per quartz disk was determined by standard plate counts on Marine agar/TSA to be $\sim 10^{8}$ cells per disk for both strains.

\section{Balloon Payload}

The MARSBOx payload $(38.1 \mathrm{~cm} \times 25.4 \mathrm{~cm} \times 63.5 \mathrm{~cm}$; mass $18 \mathrm{~kg}$ ) was built for simple mounting and integration onto the exterior of large scientific balloon gondolas (Figure 2). Biological samples were enclosed within a pressurized, shielded container (Trex-Box) (Beblo-Vranesevic et al., 2017a) with a rotatable shutter that prevented solar radiation exposure during ascent and descent (i.e., experimental initiation/termination). Covering the Trex-Box was suprasil glass: $8 \mathrm{~mm}$ thick, with a long pass cut off of $\sim 170 \mathrm{~nm}$ (with $0 \%$ transmission), and magnesium fluoride $\left(\mathrm{MgF}_{2}\right)$, with a long pass cut off of $\sim 110 \mathrm{~nm}$ (custom made by MolTech, Germany) (Figure 3B). During the balloon flight, the MARSBOx system controlled the exposure to UV radiation so that the samples were only exposed at stratosphere altitudes [Figure 4 and Supplementary Videos 1 (ascent), 2 (descent)]. Motors, gears, and the shutter were held together by a frame composed of aluminum cutouts and 3D-printed polycarbonateABS components. T-slotted 80/20 aluminum extrusions formed the framework of the payload, with detachable, white powdercoated aluminum panels on each face of the MARSBOx. Angle brackets on the back plate were used to mount the system onto the balloon gondola. The front panel data port contained one micro-USB port, six light emitting diodes (LEDs), and two key switches. One key switch was used to power on the system 

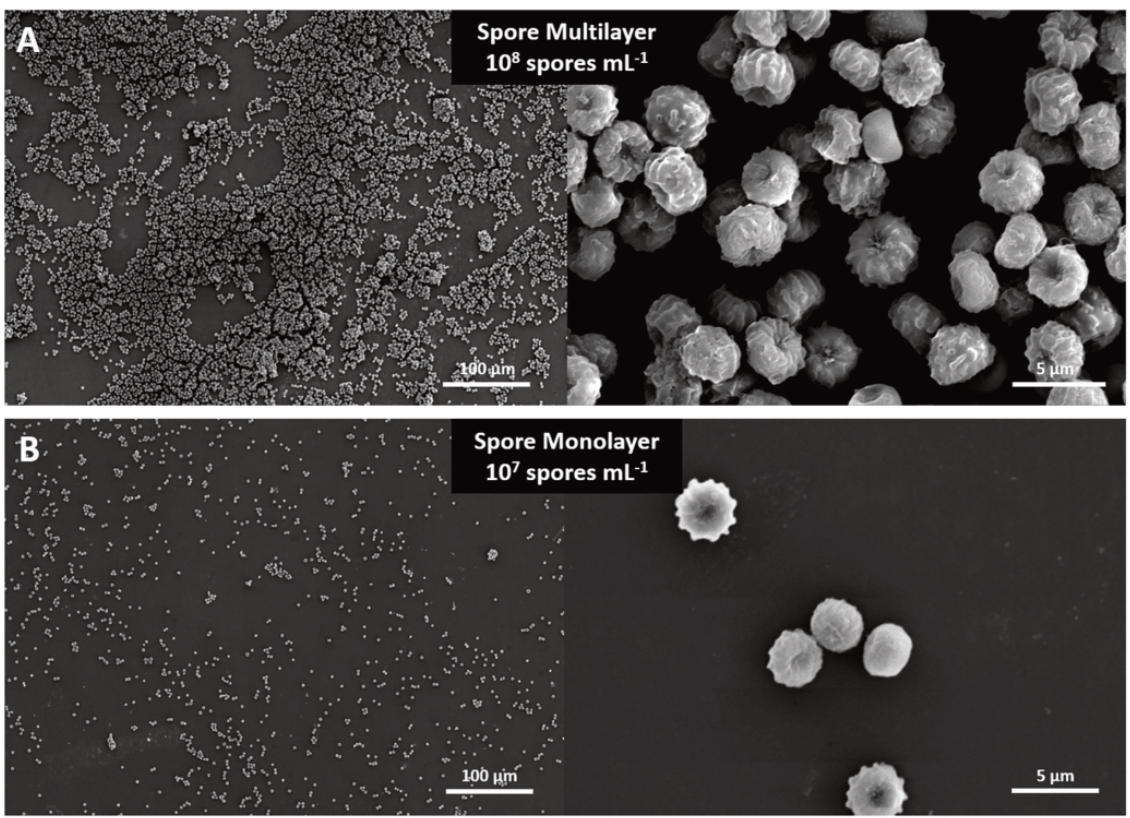

FIGURE 1 | SEM images of Aspergillus niger spores in (A) multilayer $\left(10^{8}\right.$ spores $\left.\mathrm{mL}^{-1}\right)$ and (B) monolayer $\left(10^{7}\right.$ spores $\left.\mathrm{mL}^{-1}\right)$.

and the other was used to manually rotate the shutter lid (for loading and removing Trex-Box with samples). The LEDs were programmed to indicate the state of the onboard computer's health (OSD3358, Octavo Systems) and the status of the computer's state machine, GPS receiver (GPS_FGPMMOPA6H, Adafruit Industries), camera system (Hero4 Black, GOPRO with Dash controller, CamDo), heater system, and the M-42 radiation dosimeter (Berger et al., 2019).

Other major payload components included three pressure sensors (MS5803, MEAS Switzerland; AMS5812, Analog Microelectronics; and BMP085, Bosch Sensortec), four temperature sensors, and a 9-axis Inertial Measurement Unit (IMU). While not flown on this mission, MARSBOx can carry additional instruments (e.g., UV radiometers) located behind the front panel of the payload. For the LDB \#697NT flight, power was provided by a $14.8 \mathrm{v} 25.2$ Ah lithium-ion polymer battery (CU-J141, BatterySpace) fastened in place with an ultem 3-D printed battery holder. The MARSBOx payload can also utilize a direct connection to the balloon gondola power source with an acceptable input range of $9 \mathrm{~V}-36 \mathrm{~V}$.

The design and technical details of the Trex-Box were previously described in detail (Beblo-Vranesevic et al., 2017a). Briefly, the design of the aluminum box was inspired by the EXPOSE mission series on the ISS (Rabbow et al., 2012; Rabbow et al., 2015) using a Trex-Box to control the transport of microorganisms during experiments (Beblo-Vranesevic et al., 2017a, 2020). The Trex-Box can be filled with gas and sealed, allowing for a sustained martian gas composition of $0.17 \% \mathrm{O}_{2}$, $95 \% \mathrm{CO}_{2}, 0.07 \% \mathrm{CO}, 2.6 \% \mathrm{~N}_{2}$, and $1.9 \%$ Ar during the course of the experiment at Mars-like pressures ( $\sim 7$ mbar) (Figures 3C,D). The Trex-Box enabled testing of four different microorganisms without cross-contamination (an issue reported in past balloon experiments; see Díez et al., 2020) because each organism was set in one of the four quarter sections of each layer (Figure 3A).

\section{Stratospheric Flight Experiment}

The Trex-Box consisted of two layers of an aluminum 64well sample carrier, each holding quartz disks with microbial samples (see section "Test organisms, media, and sample preparation for flight") that were either exposed to direct stratospheric UV radiation (UV-exposed, top layer) or shielded from UV (UV-shielded, bottom layer) (Table 2). The quartz disks were glued into the sample carrier using the biocompatible Vulcanizing Adhesive for Spaceflight Experiments (Wacker Chemie AG, certified by ECSS - European Cooperation for Space Standardization). For each experimental group, three quartz disks were used as ground laboratory controls and remained in normal atmospheric conditions at room temperature $\left(22^{\circ} \mathrm{C}\right)$ on the bench until analysis (Table 2).

The full mission timeline extended over 5 months including; (i) sample preparation and Trex-Box sample accommodation; (ii) MARSBOx payload integration; (iii) balloon flight; (iv) shipping and sample retrieval from Trex-Box; and (v) sample analysis. During the 5-month experimental duration, both flight and control samples were kept desiccated on quartz disks. Table 2 provides an overview of the conditions microbial samples experienced in this study. 


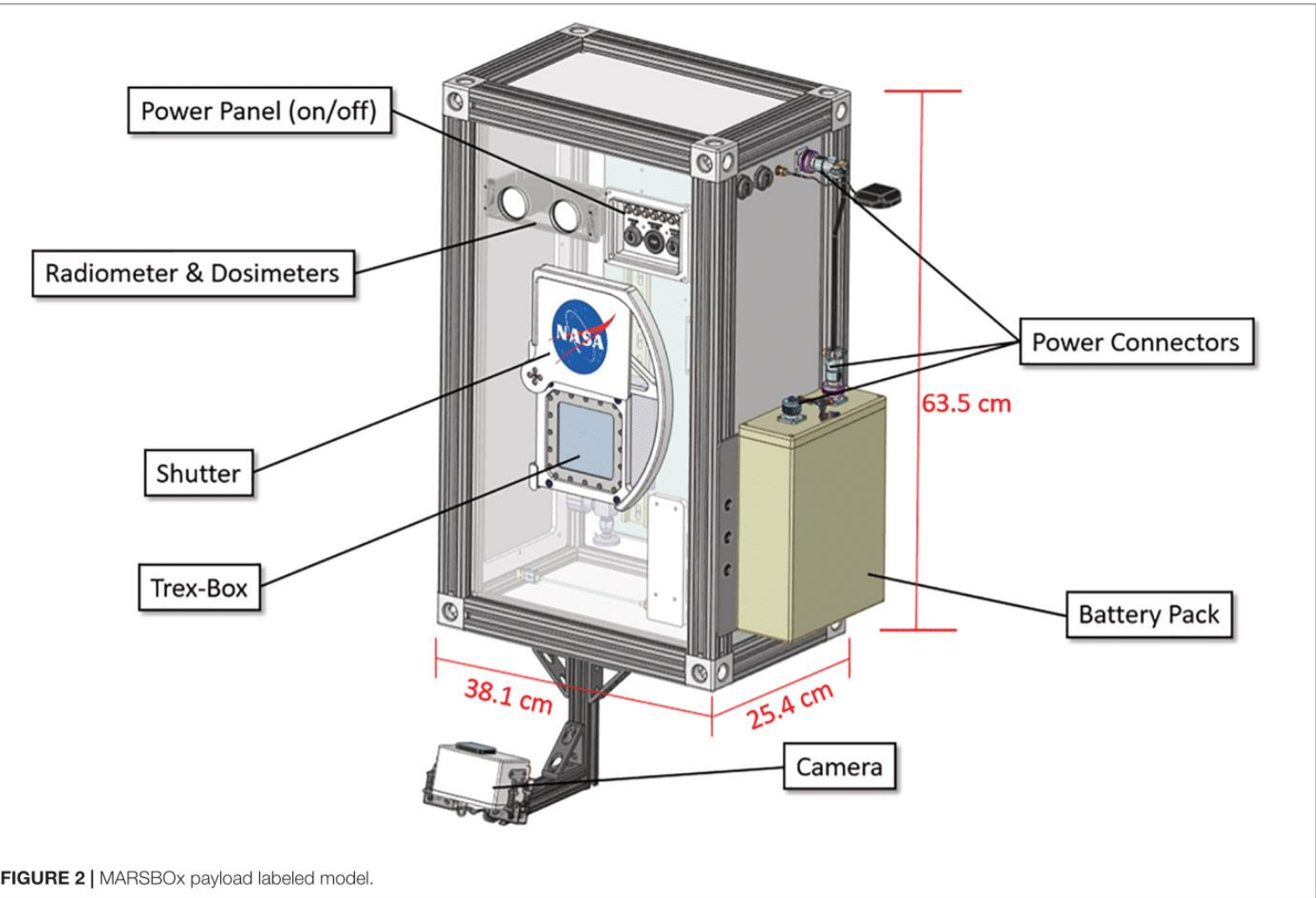

FIGURE 2 | MARSBOx payload labeled model.

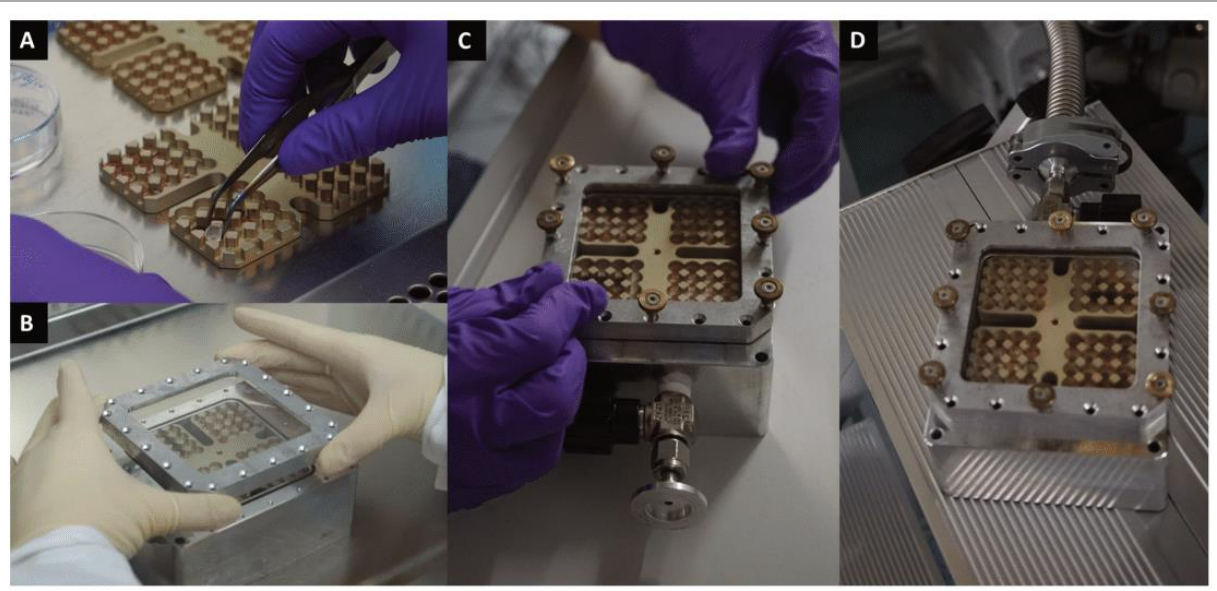

FIGURE 3 | Trex-Box sample preparation. Dimensions of the Trex-Box are $13.5 \mathrm{~cm} \times 13.5 \mathrm{~cm} \times 5.0 \mathrm{~cm}$. The container is a gastight closable stainless-steel box with one borehole which allows an exchange of internal atmosphere. (A) Quartz disks harboring the microbial samples being placed onto the Trex-Box; (B) covering the Trex-Box with a suprasil glass that allows for full UV-VIS exposure; (C) screws were used to tighten and seal the container; (D) Earth's atmosphere being replaced with Mars-gas mixture. 

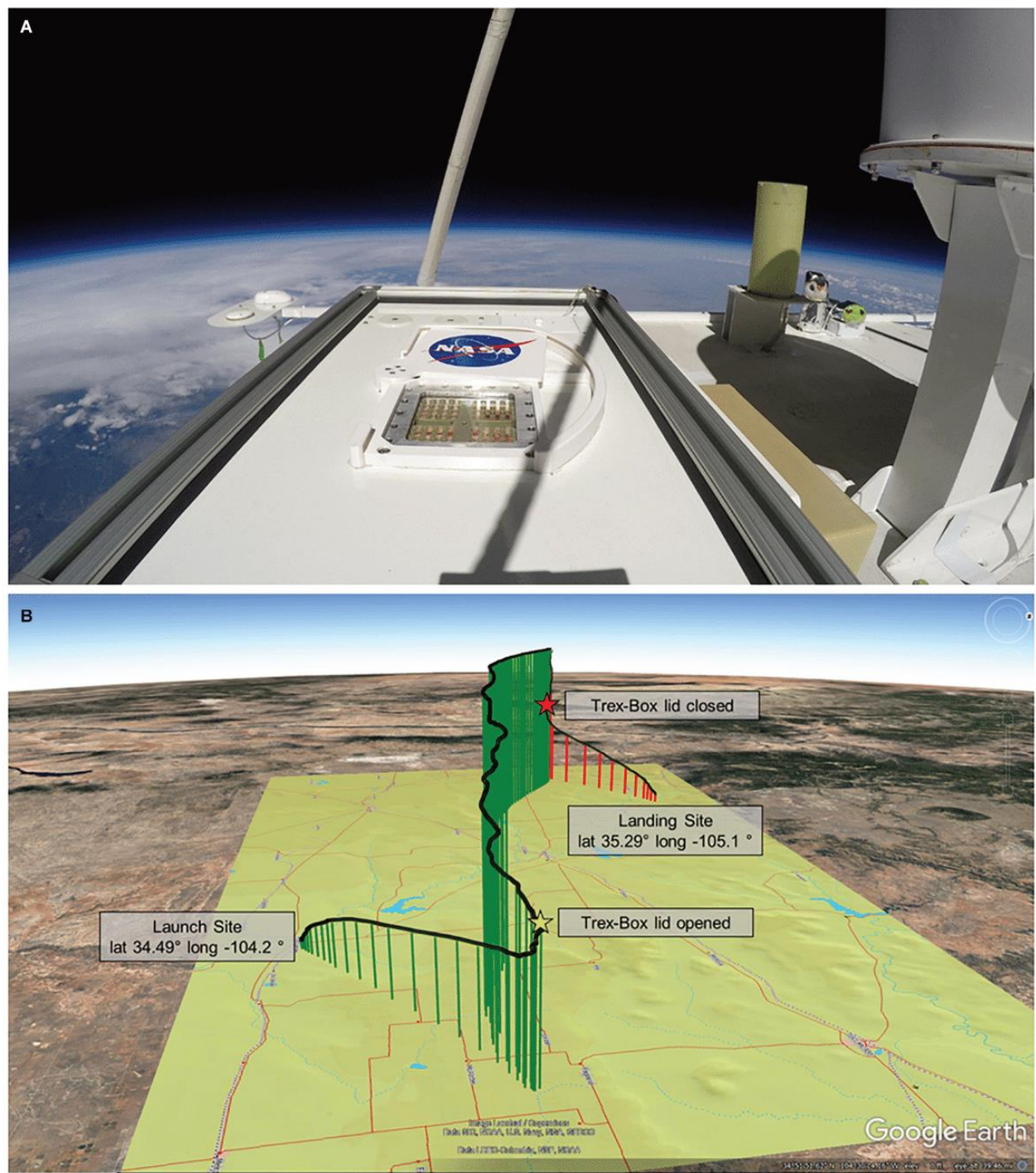

FIGURE 4 | Balloon flight (A) Image from atop the MARSBOx payload and Trex-Box in the stratosphere during the flight. (B) Balloon flight path; stars mark opening and closing of the Trex-Box lid, which corresponds to UV-VIS radiation exposure beginning and ending.

\section{Estimated UV Radiation Dose}

The total UV dose $\left[\mathrm{J} \mathrm{m}^{-2}\right]$ that the samples were exposed to during the MARSBOx mission was calculated as follows:

$$
\operatorname{Dose}\left(\mathrm{J} \mathrm{m}^{-2}\right)=\left[\text { Fluence }\left(\mathrm{mWcm}^{-2}\right) \times \operatorname{Time}(s)\right] \times 10
$$

where UV fluence $\left[\mathrm{mW} \mathrm{cm} \mathrm{cm}^{-2}\right.$ ] values for UVA-UVB (280$400 \mathrm{~nm}$ ) in the middle stratosphere were taken from a previous flight $=6 \mathrm{~mW} \mathrm{~cm} \mathrm{~cm}^{-2}$ (Caro et al., 2019), and sample exposure time [in seconds] $=19140$ s. Samples were exposed to an estimated total of $1148 \mathrm{~kJ} \mathrm{~m}^{-2}$ of UVA-UVB radiation. Previously modeled UVC (206-280 $\mathrm{nm}$ ) values by Caro et al. (2019) ranged from about $0.1-1 \mu \mathrm{W} \mathrm{cm} \mathrm{cm}^{-2}$ for the altitude flown during the MARSBOx mission $(\sim 38 \mathrm{~km})$.

\section{Ionizing Radiation Dosimetry: M-42}

In order to determine the ionizing radiation environment during the flight, a miniaturized, low-power consumption radiation detector system (M-42) was included onboard the MARSBOx 
TABLE 2 | Overview of experimental design.

\begin{tabular}{ll}
\hline Condition & Experimental treatment \\
\hline Lab Control & 5-month desiccation (air-dried, $22^{\circ} \mathrm{C}$, Earth atmosphere) \\
Bottom Layer & 5-month desiccation in Mars atmosphere and pressure. \\
(UV shielded) & Exposure to temperature fluctuation during balloon flight. \\
Top Layer & Same as bottom layer with additional exposure to \\
(UV exposed) & stratospheric UV radiation during balloon flight.
\end{tabular}

payload. The M-42 instrument (size: $142 \mathrm{~mm} \times 38 \mathrm{~mm} \times 13 \mathrm{~mm}$ ) was developed at DLR and can actively measure the absorbed dose using a silicon detector diode (Berger et al., 2019). Two batteries allow the M-42 to operate as a stand-alone radiation detector system, but for the MARSBOx flight the instrument was externally powered through the in-built micro-USB connector. Upon launch of the balloon, power was provided and the M-42 started taking measurements. Data were stored every $5 \mathrm{~min}$ on a non-volatile flash memory and upon landing the instrument was switched off.

\section{Balloon Flight Profile}

One week before flying, the MARSBOx hardware (without biological specimens loaded) was tested in a hypobaric chamber at the Columbia Scientific Balloon Facility (Palestine, TX, United States) to validate system performance and commands. The payload was then transported to the launch site at Ft. Sumner, NM, United States (lat $34.49^{\circ}$ long $-104.2^{\circ}$ ), where it was mounted onto the top portion of the LDB \#697NT gondola. After installation and prior to launch, the payload surface was sprayed with sterile air and wiped down with isopropyl alcohol. The mission carrying the MARSBOx payload was launched on 23 September 2019 at 1400 UTC, with a full video replay of the flight available here: https://www.youtube.com/watch? $=\mathrm{V}=\mathrm{Vn} 8 \mathrm{qx}$ $0 \mathrm{FmV}$. The balloon ascended for $2.5 \mathrm{~h}$ until reaching an average float altitude of $38.2 \mathrm{~km}$ where it remained for $4 \mathrm{~h}$, followed by a 35 -min descent on parachute, landing $172 \mathrm{~km}$ west of the launch site (lat $35.29^{\circ}$ long $-105.1^{\circ}$ ). Sample exposure began during ascent at $21.4 \mathrm{~km}$ with the Trex-Box shutter opening at 1521 UTC and concluded $5 \mathrm{~h}$ and $19 \mathrm{~min}$ later with the Trex-Box shutter closing during descent at $22.0 \mathrm{~km}$ at 2040 UTC (Figure 4).

The M-42 dosimeter was turned on at 1405 UTC when the payload was at $3.07 \mathrm{~km}$ and remained on until 2119 UTC at $1.75 \mathrm{~km}$ above the balloon landing site. Personnel from CSBF recovered the payload on 24 September 2019 and transported it back to the launch site facility inside a climate-controlled vehicle before shipping to NASA KSC at ambient conditions. Three weeks later, samples and instruments (Trex-Box and M-42) were removed from the MARSBOx payload and shipped to the DLR for post-flight analysis.

\section{Post-flight Processing}

After shipment arrival of the samples at the DLR, the TrexBox was opened within an anaerobic chamber (COY Laboratory products) to ensure a constant low relative humidity $(<13 \%$ relative humidity). The quartz disks harboring dried cells and spores were retrieved from the carrier and placed inside $2 \mathrm{~mL}$
Eppendorf tubes with $1 \mathrm{~mL}$ PBS respectively. For A. niger spore recovery $2 \mathrm{~mm}$ glass beads were added. The tubes were vortexed for $30 \mathrm{~s}$ to separate the cells from the disk, and the resulting suspension was used for downstream analyses.

\section{Determination of Microbial Survival via Standard Plate Counting}

The post-flight survival of the tested microorganisms was determined by standard plate counting, where serial dilutions (1:10) were plated on nutrient agar. For bacteria, TSA medium/marine medium was used; for $A$. niger minimal medium supplemented with $0.05 \%$ Triton-X was used. Agar plates were incubated for $1-3$ days at $37^{\circ} \mathrm{C}$ for bacteria and $30^{\circ} \mathrm{C}$ for fungi. Colony forming units (CFU) were counted, and the colony forming units per $\mathrm{mL}\left(\mathrm{CFU} \mathrm{mL}{ }^{-1}\right)$ were calculated. The survival fraction was calculated as $\mathrm{N} / \mathrm{N}_{0}$, in which $\mathrm{N}$ is the $\mathrm{CFU} \mathrm{mL} \mathrm{L}^{-1}$ after sample retrieval and $\mathrm{N}_{0}$ is the initial cell concentration on the quartz disk. Determination of $\mathrm{CFU} \mathrm{mL} \mathrm{m}^{-1}$ included at least three biological replicates per tested strain $(n \geq 3)$.

\section{Determination of Metabolic Activity via Resazurin Reduction}

To evaluate the potential for revival after exposure to Marslike conditions, the metabolic activity of the bacterial cells and fungal spores was measured in a 96-well-plate using resazurin reduction as an indicator (alamarBlue ${ }^{\mathrm{TM}}$ Cell Viability Reagent, Thermo Fisher ${ }^{\mathrm{TM}}$ ). In each well there was a total volume of $200 \mu \mathrm{L}(130 \mu \mathrm{L}$ of media, $50 \mu \mathrm{L}$ of dilutions, and 20 $\mu \mathrm{L}$ of alamarBlue $\left.{ }^{\mathrm{TM}}\right)$. The media used was dependent on the microorganism tested and is summarized in Table 1. The plate was incubated for $44 \mathrm{~h}$ at $30^{\circ} \mathrm{C} . \mathrm{OD}_{600}$ and $\mathrm{OD}_{570}$ were measured every $30 \mathrm{~min}$ in a Multi-Detection Microplate Reader (Infinite M200 PRO, Tecan). Orbital shaking of the plate occurred before each measurement. The percentage of reduced resazurin reagent was calculated according to the standard protocol obtained from Thermo Fisher ${ }^{\mathrm{TM}}$.

\section{Determination of $A$. niger Spore Germination}

To determine the post-flight germination of A. niger spores, spore suspensions $\left(10^{6}\right.$ spores $\left.\mathrm{mL}^{-1}\right)$ from each tested condition were drop plated $(3 \mu \mathrm{L})$, in triplicate, on $\mathrm{MM}$ agar supplemented with $0.003 \%$ yeast extract. Plates were incubated at $22^{\circ} \mathrm{C}$ for 18 $27 \mathrm{~h}$. After incubation, light microscopy was used to quantify the number of germinated $(\mathrm{G})$ and non-germinated (NG) spores. At least 200 spores were counted per replicate. Germination was calculated as the average of the G/NG ratio of each replicate per tested condition.

\section{Evaluation of Spore Cell Wall Integrity}

To test spore cell wall integrity after exposure to Mars-like environmental stress, CFUs were quantified for $A$. niger grown in the presence of an antifungal compound that acts on the cell wall (caspofungin) and a cell wall stressor (calcofluor white). Spore suspensions from the three exposure conditions (desiccated lab control, UV shielded bottom layer, and UV exposed top layer) 


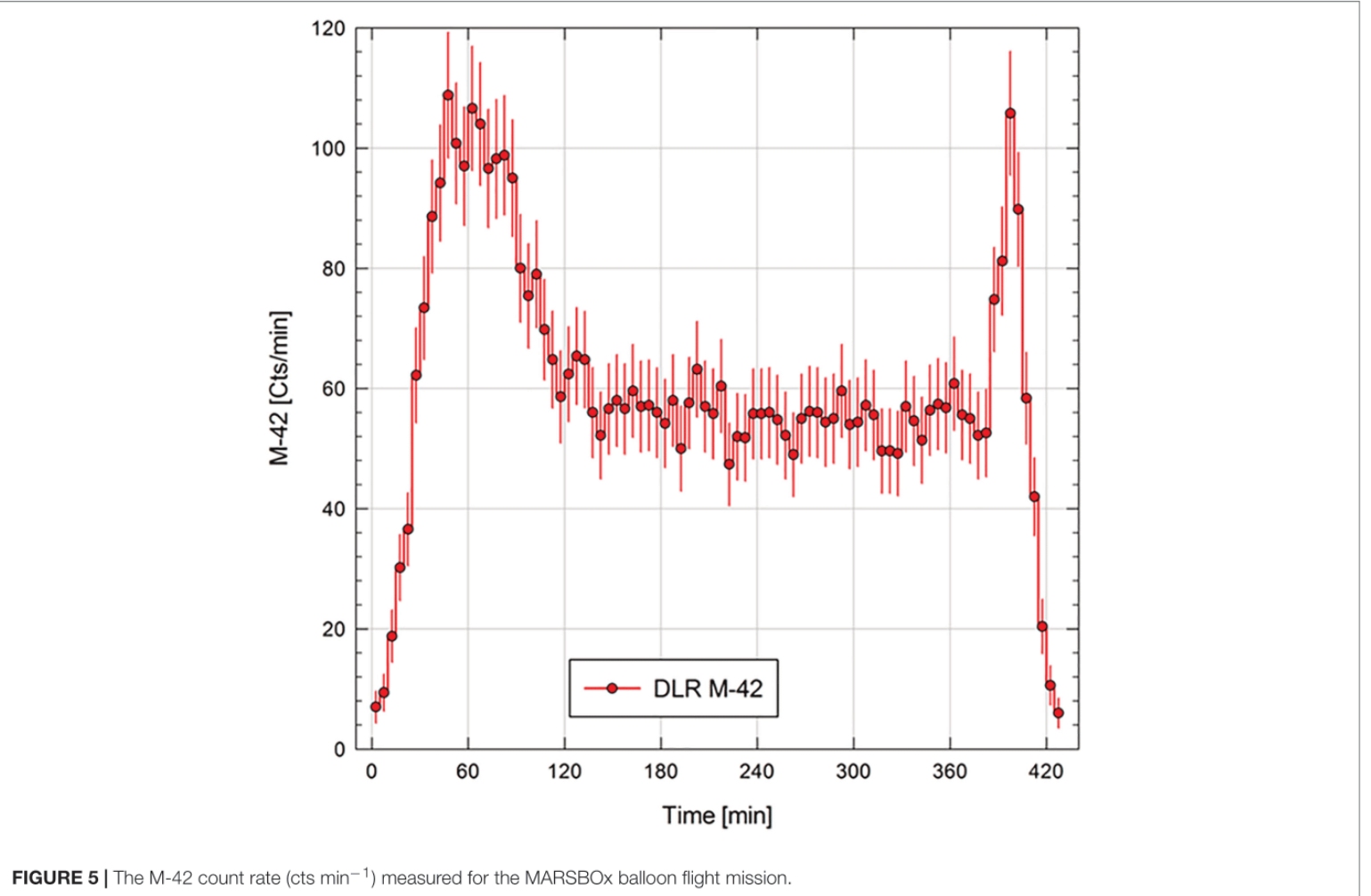

and fresh spores (as non-desiccated control) were serially diluted in a 96-well plate. For each dilution $\left(10^{-1}\right.$ to $\left.10^{-5}\right), 5 \mu \mathrm{L}$ were spotted on MM nutrient agar supplemented with $0.75 \mu \mathrm{g} \mathrm{mL}^{-1}$ caspofungin diacetate (Sigma) or $40 \mu \mathrm{g} \mathrm{mL}^{-1}$ calcofluor white (Sigma), and incubated for $2-4$ days at $30^{\circ} \mathrm{C}$.

\section{Statistical Analysis}

Survival, metabolic activity and germination data were plotted as mean values using SigmaPlot (Version 13.0, Systat Software). Error bars are presented as standard error (SE). Student's $t$-test and the non-parametric Mann-Whitney test were performed with Mean + SE to identify significant differences between each two tested conditions, per microorganism. A two-tailed $p$-value of $p \leq 0.05$ was considered significant. ANOVA analysis was also performed on survival data. A summary of $t$-test and ANOVA analysis of survival data can be found in Supplementary Tables 1-3.

\section{RESULTS}

\section{Middle Stratosphere as a Mars Analog Environment}

This study exposed different microorganisms (Aspergillus niger, Staphylococcus capitis subsp. capitis, Salinisphaera shabanensis, and Buttiauxella sp. MASE-IM-9) to a Mars analog environment.
The robust simulation of Mars environmental conditions was made possible with access to Earth's middle stratosphere onboard a scientific balloon flight, where combined conditions include elevated non-ionizing and ionizing radiation doses, low temperature, and extreme desiccation. Additionally, samples were flown inside a Trex-Box container with Mars gas composition (mostly $\mathrm{CO}_{2}$ ) and surface atmospheric pressure (510 mbar). Onboard the MARSBOx payload, microbial samples were exposed as dried cells or spores desiccated on quartz disks in two different layers: a bottom layer that was shielded from UV radiation, and a top layer that was exposed to stratospheric UV conditions. Table 3 summarizes the environmental conditions in the balloon flight compared to the generalized equatorial surface of Mars based on available measurements and models.

\section{M-42 lonizing Radiation Data}

In the following sub-section, we will only provide a snapshot of the data measured with the M-42 instrument to demonstrate proof of operations during the mission. In Figure 5, we provide the count rate of the silicon diode for the whole time the system was powered. The count rate plot shows the crossings of the Regener maximum (Regener and Pfotzer, 1935), during ascent and descent of the balloon. At cruising altitude, we saw a nearly constant count rate which results in a dose rate of $75.5 \pm 13 \mu \mathrm{Gy}$ per day. In total we measured a dose of $20.9 \mu \mathrm{Gy}$ for the 

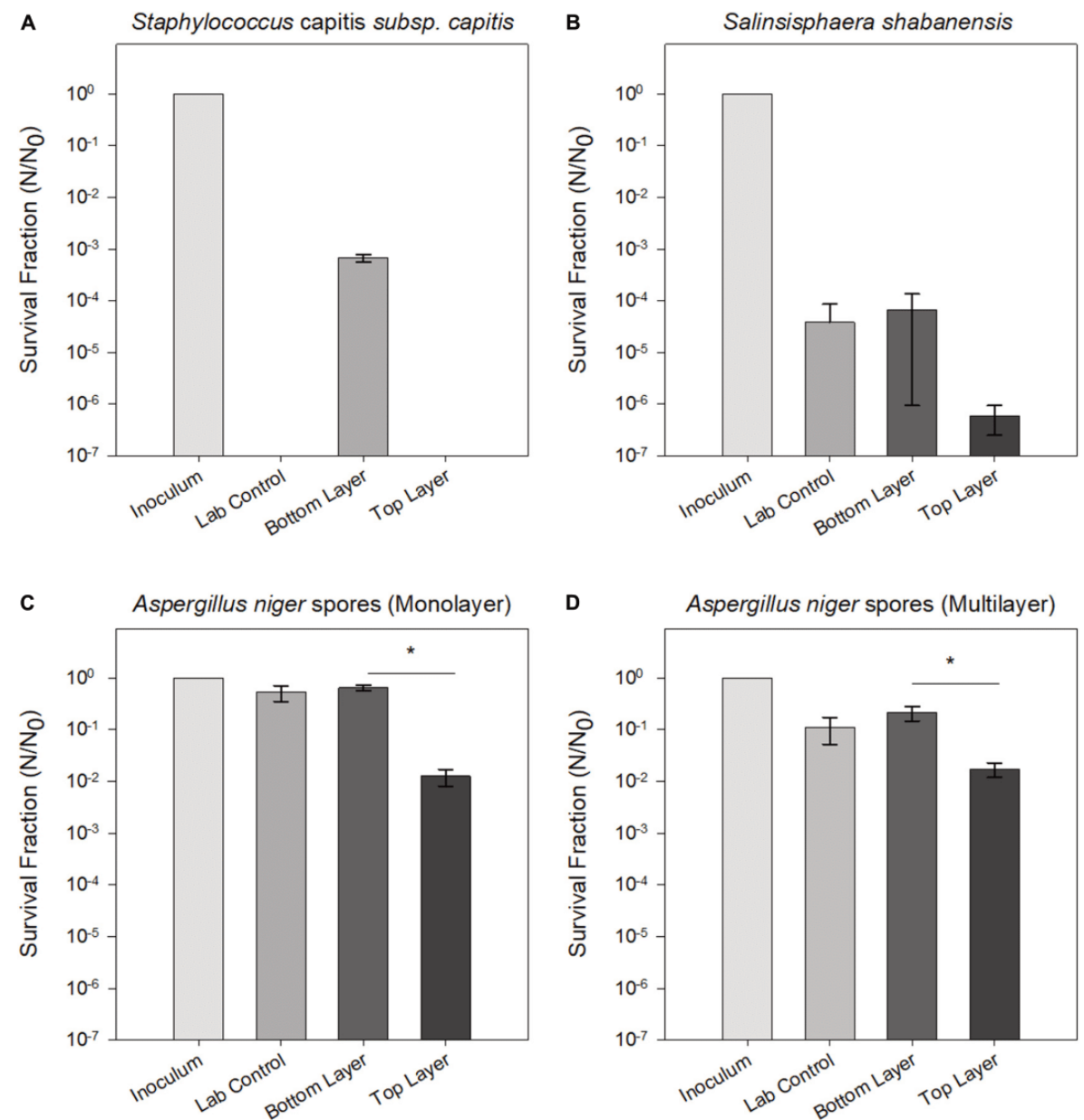

FIGURE 6 | Survival fraction (N/NO) of tested strains after exposure to Mars simulated conditions aboard the MARSBOx payload. (A) S. shabanensis (B) S. capitis subsp. capitis. (C,D) A. niger spores in monolayer (C) and in multilayer (D). Data for Buttiauxella sp. MASE-IM-9 are not shown, since no surviving cells were recovered after exposure.

whole mission, which is equivalent to around 10 days of natural background radiation received in the DLR laboratory in Cologne.

\section{Stratospheric Sunlight Reduced Microbial Survival}

Figure $\mathbf{6}$ summarizes survival fractions for exposed microorganisms in the balloon experiment. Spores from the filamentous fungus $A$. niger showed the highest endurance to the combined stresses of stratospheric radiation and simulated martian atmospheric and temperature conditions during the MARSBOx flight (Figure 6D). The extremophilic bacterium S. shabanensis tolerated desiccation but showed a decrease in survival in the UV-exposed layer of the Trex-Box (Figure 6B). The human-associated S. capitis subsp. capitis also displayed sensitivity to UV exposure, with only cells from the UV-shielded bottom layer surviving the flight experiment (i.e., still exposed to Mars gas, desiccation and temperature fluctuation). It should be noted that the laboratory control cells of $S$. capitis subsp. capitis, kept desiccated under oxic conditions, were not revivable. Buttiauxella sp. MASE-IM-9 showed no growth, even in the laboratory controls; consequently, stratosphere exposure effects could not be determined for this microorganism.

\section{Survival and Metabolic Activity of Staphylococcus capitis subsp. capitis}

For S. capitis subsp. capitis, the average of three samples $(n=3)$ from flight conditions and six samples $(n=6)$ from the laboratory control are shown in Figure 6A. In the UV-exposed samples and the laboratory controls, no surviving cells could be detected via determination of $\mathrm{CFU} \mathrm{mL}{ }^{-1}$. The UV-shielded samples showed 
TABLE 3 | Environmental conditions aboard balloon flight compared to martian conditions.

\begin{tabular}{|c|c|c|}
\hline Parameters & $\begin{array}{c}\text { Balloon } \\
\text { Flight }+ \text { Trex-Box } \\
\text { (7-38 km alt.) }\end{array}$ & $\begin{array}{c}\text { Mars } \\
\text { (at equator) }\end{array}$ \\
\hline UV fluence (280 - $400 \mathrm{~nm})$ & $\sim 6 \mathrm{~mW} \mathrm{~cm}^{-2 a}$ & $\sim 5 \mathrm{~mW} / \mathrm{cm}^{-2 b}$ \\
\hline Temperature (min.) & $-51^{\circ} \mathrm{C}$ & $-73^{\circ} \mathrm{C}$ \\
\hline Temperature (max.) & $+21^{\circ} \mathrm{C}$ & $+20^{\circ} \mathrm{C}$ \\
\hline Sample exposure duration & $5 \mathrm{~h} 19 \mathrm{~min}$ & - \\
\hline \multirow[t]{5}{*}{ Atmosphere composition } & $95 \% \mathrm{CO}_{2}$ & $96 \% \mathrm{CO}_{2}$ \\
\hline & $1.9 \% \operatorname{Ar}$ & $1.9 \% \operatorname{Ar}$ \\
\hline & $2.6 \% \mathrm{~N}_{2}$ & $1.9 \% \mathrm{~N}_{2}$ \\
\hline & $0.17 \% \mathrm{O}_{2}$ & $0.14 \% \mathrm{O}_{2}$ \\
\hline & $0.07 \% \mathrm{CO}^{c}$ & $0.07 \% \mathrm{CO}^{d}$ \\
\hline Atmospheric pressure & 5-10 mbar & 5-10 mbare \\
\hline Total UV dose (est.) & $\sim 1148 \mathrm{~kJ} \mathrm{~m}^{-2 f}$ & - \\
\hline
\end{tabular}

a Fluence rates for stratospheric UVA-UVB were measured in a previous balloon flight (Caro et al., 2019).

bschuerger et al., 2003.

"Mars gas in Trex-Box was ordered from Boggs Gases, Inc. (Titusville, FL, United States) as a commercial mixture of the top five gasses in the martian atmosphere (Schuerger et al., 2008).

'Mahaffy et al., 2013.

eBarlow, 2008.

Total estimated UV dose that samples were exposed to during the balloon flight (see section "Estimated UV radiation dose").

a significant reduction of the survival fraction by three orders of magnitude $(p=0.03)$. Metabolic activity was detected in UVshielded (bottom layer) and UV-exposed (top layer) samples, but not in the laboratory controls. Metabolic activity in the UVexposed cells was delayed in comparison to UV-shielded cells (Figure 7A). UV-shielded cells reached the maximum reduction of resazurin $(70 \%)$ after $28 \mathrm{~h}$ of incubation, whereas in UVexposed cells, the resazurin reduction was still below $70 \%$ after $44 \mathrm{~h}$ (total incubation time).

\section{Survival and Metabolic Activity of Buttiauxella sp. MASE-IM-9}

For Buttiauxella sp. MASE-IM-9, the survivability of cells dried in medium or buffer was evaluated. This strain did not survive laboratory controls or the MARSBOx flight samples. Similarly, with resazurin assay no metabolic activity was detected for Buttiauxella sp. MASE-IM-9, in any of the samples.

\section{Survival and Metabolic Activity of Salinisphaera shabanensis}

No survival differences between $S$. shabanensis cells dried in medium and $S$. shabanensis cells dried in buffer were observed. Therefore, the average of six samples $(n=6)$ was reported in Figure 6. While S. shabanensis was able to endure 5 months of desiccation, there was still an overall reduction of four orders of magnitude (laboratory control, Figure 6B). The survival for the laboratory control and the UV-shielded cells was similar ( $p=0.725)$, with UV exposure further reducing the survival fraction $(p=0.602)$ (Figure 6B). These results were supported by the metabolic activity assay with resazurin reduction in both laboratory controls and flown UV-shielded cells (Figure 7B).

\section{Survival and Metabolic Activity of Aspergillus niger Spores}

Compared to other microorganisms evaluated in our experiment, A. niger spores were the most resistant to all tested conditions (Figure 6). Two different $A$. niger spore concentrations were tested in the Trex-Box: $10^{7}$ spores $\mathrm{mL}^{-1}$ (spore monolayer) and $10^{8}$ spores $\mathrm{mL}^{-1}$ (spore multilayer); $n=3$ for each concentration (Figure 1). Survival of UV-exposed spores was reduced by two orders of magnitude compared to laboratory controls, in both spore monolayer $(p=0.001)$ and spore multilayer $(p=0.001)$. Survival of UV-shielded spores, i.e., still exposed to Mars gas, pressure and temperature, was not affected, when compared to laboratory controls, in either the monolayer $(p=0.592)$ or multilayer $(p=0.495)$ concentration (Figures 6C,D, respectively). When assessing the time taken to reach the maximum of metabolic activity, UV-exposed monolayer spores were delayed by $48 \%$ (peaking only after $43 \mathrm{~h}$ of incubation) when compared with laboratory control (peaking after $29 \mathrm{~h}$ of incubation); and multilayer spores were delayed by $38 \%$ (peaking only after $36 \mathrm{~h}$ of incubation) when compared with laboratory control (peaking after $26 \mathrm{~h}$ of incubation) as shown in Figures 7C,D, respectively.

Spore germination was delayed by $22 \%$ in UV-exposed spores, being detected only after $27 \mathrm{~h}$, versus $22 \mathrm{~h}$ of laboratory control. Germination rate was significantly lower in UV-exposed when compared to UV-shielded spores ( $p=0.01$ monolayer; $p<0.001$ multilayer) and when compared to laboratory controls ( $p=0.03$ monolayer; $p=0.08$ multilayer) (Figure 8). UV-shielded spores showed decreased ability to cope with cell wall stress (1 order of magnitude; whereas UV-exposed spores were shown to be highly sensitive to cell wall stress, 2 or more orders of magnitude) (Figure 9).

Survival, germination, and metabolic activity were compared between spore monolayer and spore multilayer (Table 4). In UVshielded martian-like conditions, the presence of a multilayer was shown to significantly decrease survival $(p=0.01)$ (Figure 6) and germination rate $(p=0.004)$ (Figure 8 ), but to significantly increase metabolic activity ( $p=0.02$ ) (Figure 7$)$. In UV-exposed, Mars-like conditions, the presence of a spore multilayer did not affect survival $(p=0.5)$ or germination rate $(p=0.1)$; but was shown to increase metabolic activity $(p=0.04)$. Multilayer spores were significantly faster in reaching the metabolic maximum than monolayer spores, in all tested conditions (Table 4).

\section{DISCUSSION}

\section{Relevance to Space Biology and Astrobiology}

In this study, bacterial and fungal strains were exposed to Marslike environmental conditions onboard the MARSBOx payload during a $7 \mathrm{~h}$ balloon flight to the middle stratosphere $(\sim 38 \mathrm{~km})$. The tested microorganisms (Aspergillus niger, Staphylococcus 

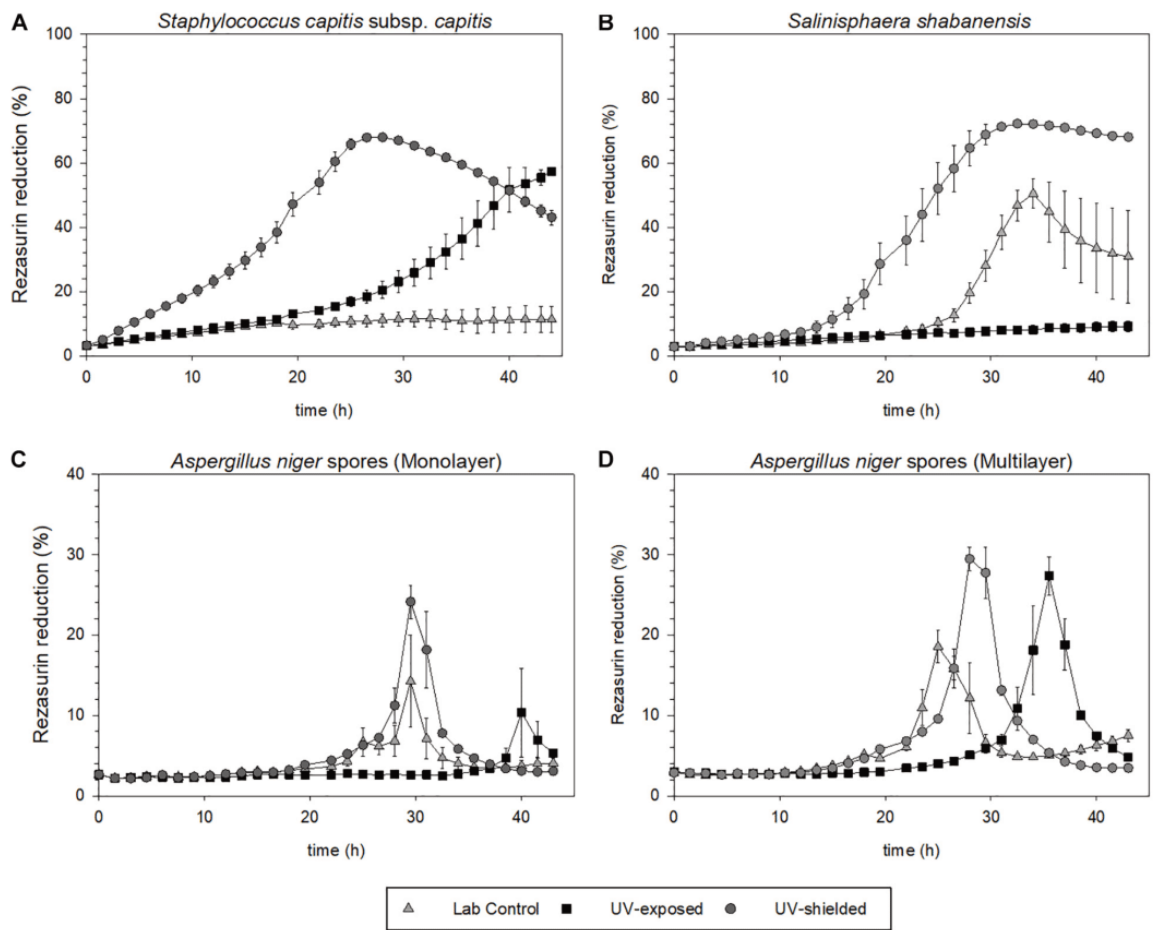

FIGURE 7 | Metabolic activity upon revival, after exposure to Mars simulated conditions aboard the MARSBOx payload. Metabolic activity is depicted as percentage of reduced resazurin. (A) S. shabanensis (B) S. capitis subsp. capitis. (C,D) A. niger spores in monolayer (C) and in multilayer (D)

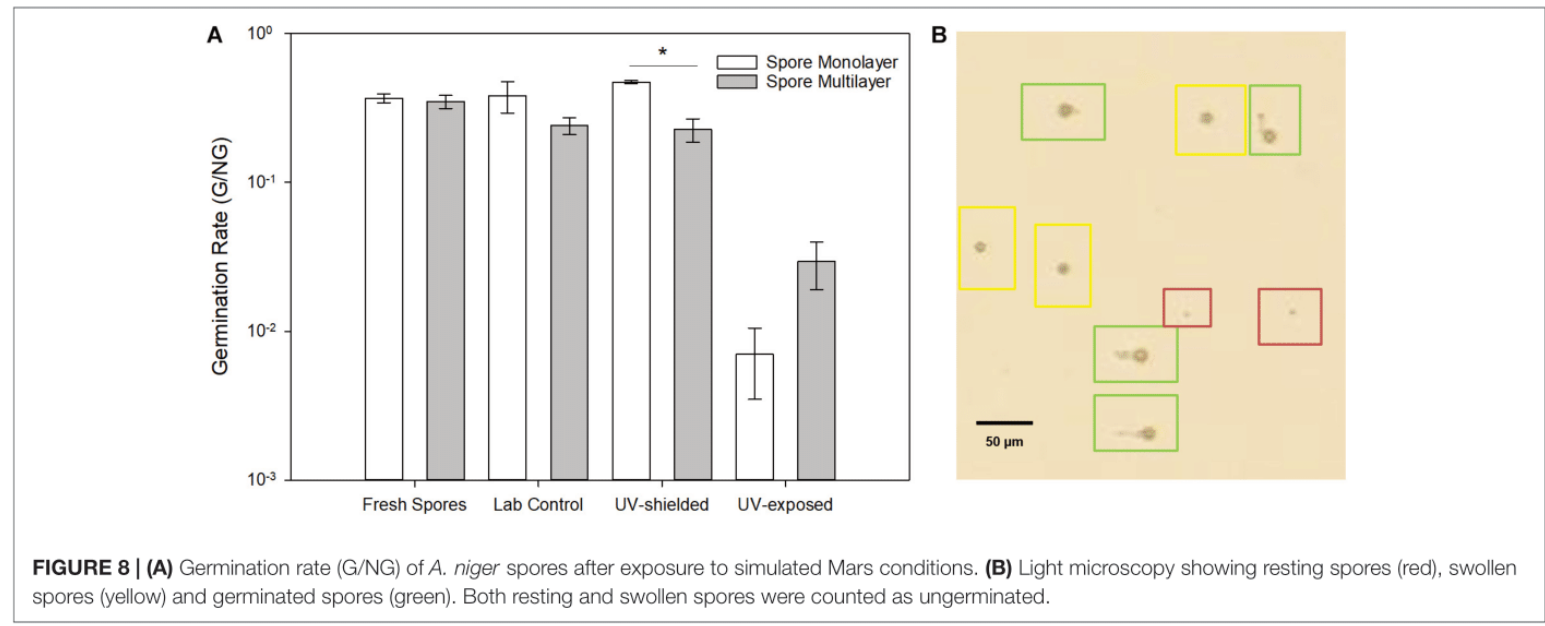

capitis subsp. capitis, Salinisphaera shabanensis, and Buttiauxella sp. MASE-IM-9) were chosen based on astrobiology and space biology relevance. Moreover, the choice of these strains was to provide a representative set of samples that are capable of demonstrating the MARSBOx experimental design as a valuable martian analog for future investigations.
To test the hypothesis that isolates from extreme Mars-analog environments on Earth would be able to survive the MARSBOx simulated martian conditions, two extremophilic bacteria were included. The bacterium $S$. shabanensis is a halophile isolated from the brine-seawater interface of the Shaban Deep at a depth of $1.3 \mathrm{~km}$ where the maximal salinity reaches 26\% (Eder 


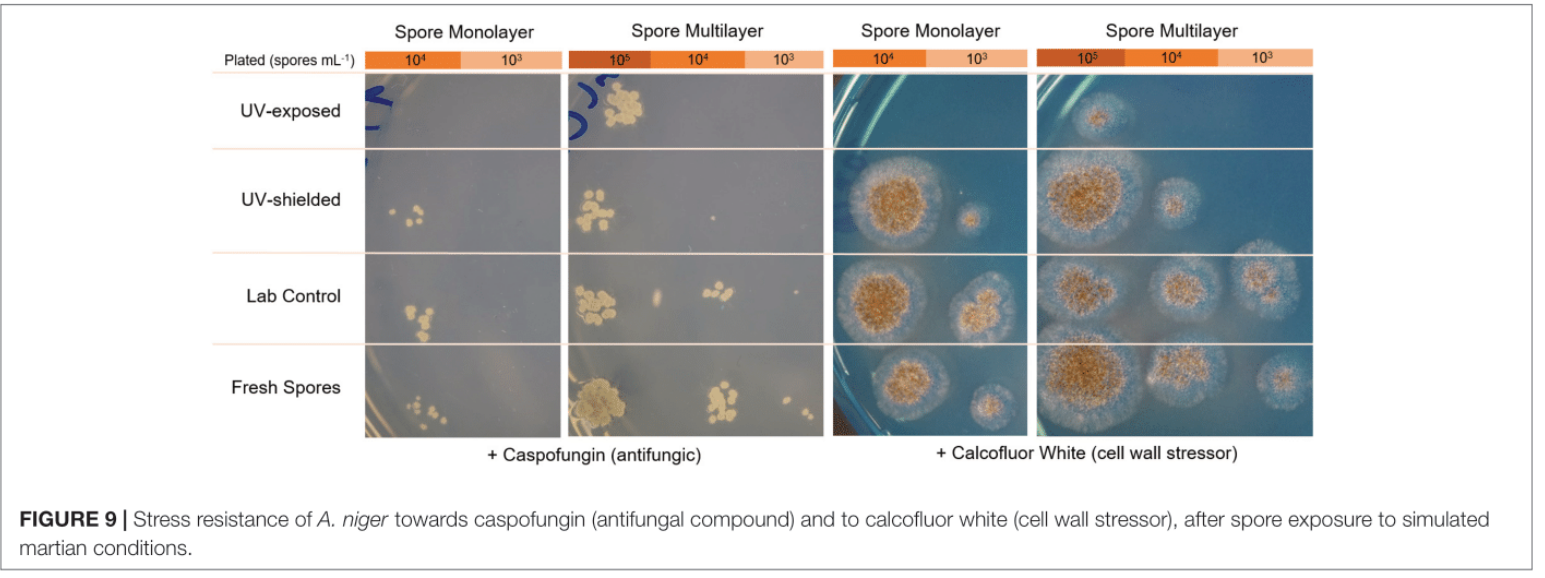

TABLE 4 | Impact of spore monolayer versus spore multilayer in A. niger resistance to Mars-like conditions.

\begin{tabular}{|c|c|c|c|c|c|}
\hline & & Monolayer & Multilayer & $f$ & $p$-value \\
\hline \multirow[t]{3}{*}{ Survival fraction ${ }^{a}$} & Lab Control & $5.3 \times 10^{-1} \pm 1.8 \times 10^{-1}$ & $1.1 \times 10^{-1} \pm 5.9 \times 10^{-2}$ & $\downarrow$ & 0.09 \\
\hline & UV shielded & $6.4 \times 10^{-1} \pm 8.0 \times 10^{-2}$ & $2.1 \times 10^{-1} \pm 6.6 \times 10^{-2}$ & $\downarrow$ & $0.01^{*}$ \\
\hline & UV exposed & $1.3 \times 10^{-2} \pm 4.5 \times 10^{-3}$ & $2.1 \times 10^{-1} \pm 5.2 \times 10^{-3}$ & $\uparrow$ & 0.5 \\
\hline \multirow[t]{3}{*}{ Metabolic max. ${ }^{b}$} & Lab Control & $20 \% \pm 5$ & $23 \% \pm 1$ & $\uparrow$ & 0.6 \\
\hline & UV shielded & $29 \% \pm 1$ & $36 \% \pm 0.1$ & $\uparrow$ & $0.002^{*}$ \\
\hline & UV exposed & $13 \% \pm 7$ & $35 \% \pm 0.5$ & $\uparrow$ & $0.04^{\star}$ \\
\hline \multirow[t]{3}{*}{ Time until max. ${ }^{c}$} & Lab Control & $29 \mathrm{~h} \pm 1.3$ & $26 \mathrm{~h} \pm 0.7$ & $\downarrow$ & 0.1 \\
\hline & UV shielded & $30 h \pm 0.3$ & $29 h \pm 0.3$ & $\downarrow$ & 0.07 \\
\hline & UV exposed & $44 \mathrm{~h} \pm 2$ & $36 \mathrm{~h} \pm 0.6$ & $\downarrow$ & 0.002 * \\
\hline \multirow[t]{3}{*}{ Germination rate ${ }^{d}$} & Lab Control & $0.38 \pm 0.09$ & $0.24 \pm 0.03$ & $\downarrow$ & 0.2 \\
\hline & UV shielded & $0.47 \pm 0.01$ & $0.23 \pm 0.04$ & $\downarrow$ & 0.004 * \\
\hline & UV exposed & $0.01 \pm 0.00$ & $0.03 \pm 0.01$ & $\downarrow$ & 0.1 \\
\hline
\end{tabular}

a Survival fraction calculated as N/NO.

${ }^{b}$ Metabolic activity maximum, measured as \% of reduced resazurin.

cIncubation time taken to reach maximum of metabolic activity (h).

${ }^{d}$ Time of incubation until metabolic peak was detected (h).

${ }^{f}$ Presence of spore multilayer has increased $(\uparrow)$ or decreased $(\downarrow)$ the measured parameter.

et al., 2002). Deep-sea brine pools have been identified as martian analogs in regards to the salinity and water activity in brines (Antunes, 2020). On the surface of Mars, brines might remain liquid at ultra-low temperatures (Toner and Catling, 2016). In addition to high salinity, the martian surface lacks oxygen and nutrients. For these reasons, the Gram-() bacterium, Buttiauxella sp. MASE-IM-9, isolated from an anoxic, nutrient-limited, and sulphidic martian analog spring in Germany (Cockell et al., 2018), was also included in our study.

An emerging body of evidence shows that spores from the fungus A. niger can withstand harsh conditions (e.g., radiation, heat, low water activity, etc.) (Singaravelan et al., 2008; Esbelin et al., 2013; Segers et al., 2018; Cortesão et al., 2020a), probably due to the roles of pigmentation, cell wall structure, and metabolic suppression, which might enable them to survive space travel on the outer surfaces of a spacecraft, and to thrive within the spacecraft's controlled habitat. Alarmingly, the inhalation of A. niger spores may cause respiratory diseases, especially when in closed indoor habitats such as the ISS, which facilitate spore dispersal (Silverman et al., 1967; Latge, 1999; Esbelin et al., 2013; Cortesão et al., 2020a,b). This motivates further study on how the species responds to spaceflight conditions, and consequent implications for astronaut health, in particular in long-term space missions. Moreover, on Earth, A. niger is used in biotechnology to produce a wide-range of useful compounds including citric acid, antibiotics, and enzymes (Meyer et al., 2011; Cairns et al., 2018). Consequently, A. niger could play an important role in human space exploration as long-duration, farreaching, missions may require biomanufacturing and resourceindependence from Earth (Silverman et al., 1967; Latge, 1999; Esbelin et al., 2013; Cortesão et al., 2020a,b).

Finally, S. capitis is a Gram-(+) bacterium commonly associated as a commensal species on human skin (Byrd et al., 2018). However, S. capitis also has the ability to cause infections in neonates and form biofilms on implants (de Silva et al., 2002; Cui et al., 2013). The Staphylococcus capitis subsp. capitis strain K1-22-23 (DSM 111179) used in this study was isolated aboard the ISS within an indoor exposure experiment (Sobisch et al., 2019). Its 
occurrence in crewed space stations, the clinical relevance and the phylogenetic proximity to other clinically relevant staphylococcal species make S. capitis a useful model organism to study the effects of space conditions on opportunistic human pathogens, and to identify potential risks of crew infection (Xiao et al., 2019).

\section{Radiation Levels in the Middle Stratosphere}

Life on Earth is protected from low-wavelength UV radiation (100-280 nm) by atmospheric ozone (Horneck et al., 2010) Above the concentration of atmospheric ozone, where large scientific balloon missions float, UV radiation levels nearly match those expected on the surface of equatorial Mars. For instance, Caro et al. (2019) recently measured an average instantaneous UVA-UVB flux of $\sim 6 \mathrm{~mW} \mathrm{~cm}$ ch $^{-2}$ on meteorological balloon mission flown to the middle stratosphere; the total combined dose measured was $1.9 \mathrm{~kJ}$. In comparison, $\sim 5 \mathrm{~mW} \mathrm{~cm} \mathrm{~cm}^{-2}$ was the reported value to be expected at the surface of Mars for UVA-UVB according to calculations from Schuerger et al. (2003). Surprisingly few UVC measurements have been obtained for Earth's middle stratosphere but Caro et al. (2019) modeled an expected range of $0.1-1 \mu \mathrm{W} \mathrm{cm}{ }^{-2}$ for altitudes above the ozone layer. Besides intense UV radiation (derived levels in this study), we measured ionizing radiation in the middle stratosphere with the M-42 active dosimeter. This was the first successful M42 flight test in preparation for the dosimetry suite onboard the upcoming Matroshka AstroRad Radiation Experiment on the NASA Artemis I mission (Berger et al., 2019). At the $\sim 38 \mathrm{~km}$ float altitude on the MARSBOx mission, the dose rate was almost constant, around $75.5 \pm 13 \mu \mathrm{Gy} \mathrm{day}^{-1}$. In comparison, the dose rate measured on the surface of Mars for the same time period would approach $260 \mu \mathrm{Gy} \mathrm{day}^{-1}$. A more comprehensive radiation biophysics analysis (e.g., Monte Carlo calculations) will be reported later alongside additional balloon flight measurements from a joint NASA-DLR long-duration Antarctic mission flown in December 2019. In the meantime, we point readers interested in the ionizing radiation levels of the middle stratosphere over New Mexico to the RaD-X mission results from Mertens (2016).

\section{Bacterial Survival}

The endurance of $S$. shabanensis, a non-pigmented halophile, to the (derived) UV dose of $1148 \mathrm{~kJ} \mathrm{~m}^{-2}$ during the balloon flight was unanticipated considering the original isolation source for the species was a deep-sea brine pool with no direct illumination from sunlight. Our stratosphere balloon mission results for S. shabanensis (partial resistance to UV exposure) reveal a wide variability in UV response based on the model bacterium studied. It was previously reported that even vegetative Escherichia coli cells can persist for 7 days under simulated Mars conditions, even when exposed to $8 \mathrm{~h}$ of UVC irradiation (200$280 \mathrm{~nm}$ ) at a fluence of $3.6 \mathrm{~W} \mathrm{~m}^{-2}$ per day (Berry et al., 2010). Besides innate physiological differences, survival rates might also be influenced by the degree to which the UV dose was attenuated. With past laboratory- and flight-based experiments embedding microorganisms in different substrates - including
Mars analog soils - the effects of UV (alone) can be difficult to assess (Rettberg et al., 2004; Wadsworth and Cockell, 2017). Cell layering (discussed later in section " $A$. niger spores survive Mars-like conditions") also likely plays a role in variable survival outcomes, as reported with past stratosphere exposure studies (Khodadad et al., 2017). We expected S. capitis subsp. capitis to be more tolerant to radiation in the stratosphere because of its natural occurrence on human skin (Byrd et al., 2018), where direct illumination from sunlight would be common. For instance, the average UV dose causing erythema (abnormal redness of the skin) of Americans is approximately $25 \mathrm{~kJ} \mathrm{~m}^{-2}$ per year (Godar et al., 2001).

Whether the tested bacterial strains would be able to survive in a real Mars-surface environment depends on various aspects; however, access to UV-shielding will certainly play a major role. This was seen in our study, as both the halophilic bacterium S. shabanensis, and the human skin associated bacterium S. capitis subsp. capitis survived the UV-shielded Mars-like environment during the balloon flight. In the event that these bacteria are brought to Mars, either in robotic missions for astrobiological research purposes; or by accident through crewled contamination in space missions (Avila-Herrera et al., 2020) our results suggest that bacterial bioburden embedded deep inside of spacecraft sent to Mars might remain viable for longer periods of time $(>5 \mathrm{~h})$.

\section{Bacterial Desiccation Tolerance in Mars-Like Conditions}

Desiccation can be a stressful condition for cells, where the accumulation of reactive oxygen species and irreversible changes in lipids, proteins, and nucleic acids can lead to death (Cox, 1993; Dose and Gill, 1995). Some microorganisms can tolerate extreme desiccation by ceasing metabolic activity in a state of anhydrobiosis (Glasheen and Hand, 1988; Wright, 1989; Potts, 1994). Our results for the bacterial species S. shabanensis showed survival under long-term desiccation and sunlight exposed flight conditions. The survival for the laboratory control and the UVshielded organisms are similar, which may indicate a desiccation sensitivity of this strain. Cells of S. capitis subsp. capitis K1-2-223 were inactivated from the flight UV-exposure but did partially survive the UV-shielded layer of the Trex-Box. Interestingly, the desiccated laboratory controls for $S$. capitis subsp. capitis did not survive the experiment, indicating that long-term resistance to desiccation was only possible when cells were under the Marslike atmosphere and not when kept in Earth atmosphere. The results from the balloon flight warrant further investigation to determine if a Mars gas mixture has an impact on the stability of some bacterial species. For instance, it is known that the presence of oxygen can decrease the survivability of prokaryotes during desiccation (Potts, 1994; Vriezen et al., 2007; BebloVranesevic et al., 2017b) and in additional experiments we observed that the survival of $S$. capitis subsp. capitis K1-2-223 increased when desiccation occurred in anoxic conditions (data not shown). Finally, the last bacterial species flown in this experiment, Buttiauxella sp. MASE-IM-9, did not survive long-term desiccation. Therefore, no surviving cells could be 
detected in the balloon flight samples or from laboratory controls. This negative result was consistent with previous Mars analog experiments where Buttiauxella sp. MASE-IM-9, a facultative anaerobe, survived a maximum of 3 months of desiccation (Beblo-Vranesevic et al., 2018, 2020).

\section{A. niger Spores Survive Mars-Like Conditions}

While bacterial cells were sensitive to UV exposure in the middle stratosphere, in addition to long-term desiccation, $A$. niger spores were highly resistant to all tested conditions. Laboratory controls demonstrated $A$. niger spore endurance to 5 -month desiccation, when air-dried and kept at room temperature $\left(22^{\circ} \mathrm{C}\right)$ at the bench, under Earth atmospheric conditions. Spores of $A$. niger shielded from UV (bottom layer) endured a 5-month desiccation within Mars-like atmosphere and pressure, with additional exposure to extreme temperature fluctuations during the balloon flight $\left(-51^{\circ} \mathrm{C}\right.$ to $\left.+21^{\circ} \mathrm{C}\right)$. Finally, spores of $A$. niger exposed to UV (top layer) withstood over $5 \mathrm{~h}$ of full simulation of Mars environmental conditions, i.e., exposed to Mars gas, atmosphere, temperature fluctuation, and to a total estimated UVA-UVB dose of $1148 \mathrm{~kJ} \mathrm{~m}^{-2}$ during the balloon flight. A previous study testing A. niger spore survival to desiccation and solar radiation was done in an experimental setting similar to this study: $A$. niger spore monolayers were dried in glass disks and exposed to $\sim 16 \mathrm{~kJ}$ $\mathrm{m}^{-2}$ of UVB (280-320 nm); the specimens were highly resistant, with $24 \% \pm 5 \%$ survival (Dose et al., 2001). The extraordinarily high level of resistance of $A$. niger spores to UVC radiation (LD90 value of $1038 \mathrm{~J} \mathrm{~m}^{-2}$ ) has been previously reported by Cortesão et al. (2020a). Another balloon-flown study (i.e., same launch site and season; different year) with pigmented spores of the fungus Fuligo spectica showed that these remained viable after a $9 \mathrm{~h}$ exposure to conditions in the stratosphere (Díez et al., 2020). In the Aspergillus genus, secondary metabolites e.g., DHN-melanin (a pigment) and fumiquinazoline might be associated with UV protection roles (Blachowicz et al., 2020).

The ubiquitous presense of $A$. niger spores in human indoor-closed habitats, and their high resistance to outer space conditions, suggests these will likely travel with us to Mars. A contamination scenario, several factors might affect the survivability potential of $A$. niger spores in a Mars-surface environment. Some are intrinsic to the spores, e.g., molecular mechanisms such as DNA repair systems; or structural protection mechanisms such as the thick cell wall (Latgé et al., 2017). Whereas other factors are external, for instance, shielding from the spacecraft surfaces or martian regolith. An important factor known to impact survival outcomes is the starting cell concentration (Khodadad et al., 2017). To test this, we compared A. niger responses to the stratosphere as either a spore monolayer $\left(10^{7}\right.$ spores $\left.\mathrm{ml}^{-1}\right)$ or a spore multilayer $\left(10^{8}\right.$ spores $\mathrm{ml}^{-1}$ ) (Table 4). When exposed to UV-shielded conditions, a higher starting spore concentration (multilayer) influenced all primary measures (i.e., survival fraction, spore germination, and metabolic activity). Unexpectedly, spores in a multilayer yielded a decreased survival in laboratory controls and UV shielded conditions, as well as a decreased germination rate in all tested conditions, when compared with spores in a monolayer. These discrepancies might simply be due to incomplete removal of biomass from the quartz disks or due to the presence of hyphae fusion in germination test plates; either of which could lead to undercounting.

Moreover, in A. niger spores, the cell wall is a highly complex structure that plays an important role in protecting the spores from extracellular environmental stress. The spore cell wall is composed mainly of polysaccharides ( $\alpha$-glucans $\beta$-glucans), galactomannan, and chitin; and is surrounded by a rodlet layer with hydrophobic surface proteins, and a melanin layer. When germinating into vegetative cells (hyphae), the spore cell wall is remodeled and no longer provides protection to extreme conditions (Latgé et al., 2017). Considering environmental changes typically act first on the cell wall, we evaluated how Mars-like conditions might alter A. niger spore cell wall integrity. All tested spores were able to revive (i.e., germinate) and grow in media supplemented with cell wall stressors: calcofluor white or caspofungin. Calcofluor white is a non-specific fluorochrom that can bind to $1,3-$ and $1,4-\beta$ polysaccharides on chitin and cellulose, inhibiting chitin microfibril assembly and cell wall integrity (Fiedler et al., 2014).

\section{CONCLUSION}

To date, a variety of terrestrial analogs and simulation chambers have been used to predict outcomes for microbial exposure to Mars-like conditions. Most often, such investigations use elevated radiation and desiccation paired with low pressure and temperature alongside a Mars gas mixture (Jensen et al. 2008; Schuerger et al., 2008; Motamedi et al., 2015). However, few studies can simultaneously recreate a multi-factor Mars environment. The renewed focus on Mars robotic and human exploration e.g., Mars 2020, Mars Sample Return (NASA, 2020) and ExoMars 2022 (ESA, 2020) amplifies the need for additional Mars analog studies in the coming years. In this study we:

- reported the use of a new scientific payload (MARSBOx) for stratospheric balloon missions allowing access to a wideranging Mars analog environment with natural ionizing and non-ionizing radiation;

- demonstrated a successful experimental set-up, of the Trex-Box and MARSBOx combined, where four different microorganisms could be tested, in dried conditions, throughout a 5-month period, without cross-contaminations.

- showed that the extremophilic bacterium S. shabanensis and the human skin-associated bacterium $S$. capitis subsp. capitis survived the UV-shielded Mars-like environment during the balloon flight, suggesting that bioburden embedded deep inside of spacecraft sent to Mars might remain viable for longer periods of time;

- revealed that highly pigmented spores from the fungus A. niger would survive, in a Mars-like middle stratosphere environment for $>5 \mathrm{~h}$ of UV exposure, even as a spore monolayer $\left(10^{6}\right.$ spores $\left.\mathrm{mL}^{-1}\right)$, i.e., with no self-shielding.

Taken together, we conclude pigmented fungal spores might be considered some of the most likely forward contaminants to 
survive if inadvertently delivered to Mars. Our results underscore the importance of including fungal spores in Mars forward contamination studies and relevant planetary protection policies, which currently restrict surface bioburden of $\leq 3 \times 10^{5}$ bacterial endospores for robotic lander systems that are not carrying instruments to investigate extant martian life (category IVa) (COSPAR, 2020). Moreover, fungal spore sensitivity to extreme heat or to high doses of combined sources of space radiation, as well as to other factors that affect survivability (e.g., regolith reactive compounds or regolith shielding), should be further evaluated to better assess the forward contamination potential in Mars analog environments.

\section{DATA AVAILABILITY STATEMENT}

The original contributions presented in the study are included in the article/Supplementary Material, further inquiries can be directed to the corresponding author/s.

\section{AUTHOR CONTRIBUTIONS}

$\mathrm{MC}, \mathrm{KS}, \mathrm{SK}$, and KB-V performed the microbial experiments, analyzed the data, and wrote the manuscript. DS, SW, SV, ER, and RM contributed to the conception and design of the study, data analyses and manuscript preparation. DS, ML, LJ, and PJ prepared and performed the balloon flight mission and contributed to manuscript preparation. TB contributed with the M-42 experiment and manuscript preparation. All authors contributed to the article and approved the submitted version.

\section{FUNDING}

KS, KB-V, TB, and RM were supported by the DLR grant FuEProjekt "ISS LIFE” (Programm RF-FuW, Teilprogramm 475). MC was supported by the DLR/DAAD Research Fellowship Doctoral Studies in Germany, 2017 (57370122). DS funding was from NASA Space Biology and NASA Planetary Protection

\section{REFERENCES}

Acuña, M., Connerney, J., Wasilewski, P., Lin, R., Mitchell, D., Anderson, K. et al. (2001). Magnetic field of Mars: summary of results from the aerobraking and mapping orbits. J. Geophys Res. Planets 106, 23403-23417. doi: 10.1029/ 2000je001404

Antunes, A. (2020). Astrobiology: Current, Evolving and Emerging Perspectives. Poole: Caister Academic Press.

Antunes, A., Eder, W., Fareleira, P., Santos, H., and Huber, R. (2003). Salinisphaer shabanensis gen. nov., sp. nov., a novel, moderately halophilic bacterium from the brine-seawater interface of the Shaban Deep. Red Sea. Extremophiles 7, 29-34. doi: 10.1007/s00792-002-0292-5

Avila-Herrera, A., Thissen, J., Urbaniak, C., Be, N. A., Smith, D. J., Karouia, F., et al. (2020). Crewmember microbiome may influence microbial composition of ISS habitable surfaces. PLoS One 15:e231838 doi: 10.1371/journal.pone.0231838

Barlow, N. G. (2008). Mars: An Introduction to its Interior, Surface and Atmosphere. Cambridge: Cambridge University Press.
Research grants. SW was supported by the NASA Postdoctoral Program (NPP) as a fellow.

\section{ACKNOWLEDGMENTS}

We would like to thank the NASA Balloon Program Office (Wallops Flight Facility) and the staff at the Columbia Scientific Balloon Facility for our chamber test activities in Palestine, Texas, and our balloon flight opportunity in Ft. Sumner, New Mexico. We would also like to thank Andrea Schröder for the technical support in sample accommodation on the Trex-Box. Furthermore, we thank Dr.-Ing. Marcel Fiebrandt for technical support and providing of the scanning electron microscope at the Chair of General Electrical Engineering and Plasma Technology (AEPT) at the Ruhr-University Bochum.

\section{SUPPLEMENTARY MATERIAL}

The Supplementary Material for this article can be found online at: https://www.frontiersin.org/articles/10.3389/fmicb. 2021.601713/full\#supplementary-material

Supplementary Figure 1 | Environmental data from the flight via CSBF (outside temperature and pressure and altitude).

Supplementary Table 1| Student's t-test was used to identify significant differences between two tested conditions of the survival data using raw data (CFU/mL). Two-tailed $p$-value is presented. Equal variances assumed.

Supplementary Table 2 | Student's t-test was used to identify significant differences between two tested conditions of the survival data (Survival Fraction $\mathrm{N} / \mathrm{N}_{0}$ ) using mean values and standard error. Two-tailed $p$-value is presented. Equal variances assumed.

Supplementary Table 3 | ANOVA on Ranks followed by Kruskal-Wallis and Dunn's test were used to identify significant differences in the survival data (CFU/mL).

Supplementary Video 1 | Rotatable shutter opening to start sample exposure to UV radiation, once stratosphere altitudes were reached.

Supplementary Video 2 | Rotatable shutter closing to stop sample exposure to UV radiation, in preparation for descent.

Be, N. A., Avila-Herrera, A., Allen, J. E., Singh, N., Checinska Sielaff, A., Jaing, C., et al. (2017). Whole metagenome profiles of particulates collected from the International Space Station. Microbiome 5:81.

Beblo-Vranesevic, K., Bohmeier, M., Perras, A. K., Schwendner, P., Rabbow, E., Moissl-Eichinger, C., et al. (2017a). The responses of an anaerobic microorganism, Yersinia intermedia MASE-LG-1 to individual and combined simulated Martian stresses. PLoS One 12:e0185178. doi: 10.1371/journal.pone. 0185178

Beblo-Vranesevic, K., Bohmeier, M., Perras, A. K., Schwendner, P., Rabbow, E., Moissl-Eichinger, C., et al. (2018). Lack of correlation of desiccation and radiation tolerance in microorganisms from diverse extreme environments tested under anoxic conditions. FEMS Microbiol. Lett. 365: fny044.

Beblo-Vranesevic, K., Bohmeier, M., Schleumer, S., Rabbow, E., Perras, A. K. Moissl-Eichinger, C., et al. (2020). Impact of simulated martian conditions on (facultatively) anaerobic bacterial strains from different Mars analogue sites. Curr. Issues Mol. Biol. 38, 103-122. doi: 10.21775/cimb.038.103 
Beblo-Vranesevic, K., Galinski, E. A., Rachel, R., Huber, H., and Rettberg, P. (2017b). Influence of osmotic stress on desiccation and irradiation tolerance of (hyper)-thermophilic microorganisms. Arch. Microbiol. 199, 17-28. doi 10.1007/s00203-016-1269-6

Beck-Winchatz, B., and Bramble, J. (2014). High-altitude ballooning student research with yeast and plant seeds. Gravit Space Res. 2, $117-127$.

Berger, T., Marsalek, K., Aeckerlein, J., Hauslage, J., Matthiä, D., Przybyla, B. et al. (2019). The German Aerospace Center M-42 radiation detector - A new development for applications in mixed radiation fields. Rev. Sci. Instrum. 90:125115. doi: 10.1063/1.5122301

Berry, B. J., Jenkins, D. G., and Schuerger, A. C. (2010). Effects of simulated Mars conditions on the survival and growth of Escherichia coli and Serratio liquefaciens. Appl. Environ. Microbiol. 76, 2377-2386. doi: 10.1128/aem.02147-

Binder, A. B. (1966). Mariner IV: analysis of preliminary photographs. Science 152, 1053-1055. doi: $10.1126 /$ science.152.3725.1053

Blachowicz, A., Raffa, N., Bok, J. W., Choera, T., Knox, B., Lim, F. Y., et al. (2020). Contributions of spore secondary metabolites to UV-C protection and virulence vary in different Aspergillus fumigatus strains. Mbio 11, e3415-e3419.

Bos, C. J., Debets, A. J. M., Swart, K., Huybers, A., Kobus, G., and Slakhorst, S. M. (1988). Genetic analysis and the construction of master strains for assignment of genes to six linkage groups in Aspergillus niger. Curr. Genet. 14, 437-443. doi: $10.1007 /$ bf00521266

Byrd, A. L., Belkaid, Y., and Segre, J. A. (2018). The human skin microbiome. Nat Rev. Microbiol. 16:143.

Cairns, T. C., Nai, C., and Meyer, V. (2018). How a fungus shapes biotechnology: 100 years of Aspergillus niger research. Fungal. Biol. Biotechnol. 5:13.

Caro, T. A., Wendeln, M., Freeland, M., Bryan, N., Waters, S. M., McIntyre A., et al. (2019). Ultraviolet light measurements $(280-400 \mathrm{~nm})$ acquired from stratospheric balloon flight to assess influence on bioaerosols. Aerobiologia 35 , 771-776. doi: 10.1007/s10453-019-09597-9

Catling, D. C. (2009). "Atmospheric evolution, mars," in Encyclopedia of Paleoclimatology and Ancient Environments, ed. V. Gornitzs (Dordrecht: Springer Netherlands), 66-75. doi: 10.1007/978-1-4020-4411-3_17

Checinska, A., Probst, A. J., Vaishampayan, P., White, J. R., Kumar, D., Stepanov, V., et al. (2015). Microbiomes of the dust particles collected from the International Space Station and Spacecraft Assembly Facilities. Microbiome 3:50.

Clancy, R. T., and Muhleman, D. O. (1993). "Ground-based microwave spectroscopy of the Earth's stratosphere and mesosphere," in Atmospheri Remote Sensing by Microwave Radiometry, ed. M. A. Janssen (New York, NY John Wiley), 335-382.

Clark, G. Q., and McCoy, J. G. (1965). Measurement of stratospheric temperature. J. Appl. Meteorol. 4, 365-370. doi: 10.1175/1520-0450(1965)004<0365:most $>2$. $0 . \mathrm{co} ; 2$

Clarke, J. D., and Persaud, R. (2004). "Expedition two: a multi-goal Mars analogue expedition to the Arkaroola region, Australia," in Proceedings of the 2004 Australian Mars Exploration Conference, (Sydney).

Cockell, C., Schwendner, P., Perras, A., Rettberg, P., Beblo-Vranesevic, K. Bohmeier, M., et al. (2018). Anaerobic microorganisms in astrobiological analogue environments: from field site to culture collection. Astrobiology 17 314-328. doi: $10.1017 / \mathrm{s} 1473550417000246$

Cockell, C. S., Bush, T., Bryce, C., Direito, S., Fox-Powell, M., Harrison, J. P., et al. (2016). Habitability: a review. Astrobiology 16, 89-117.

Coleman, J. S. M., and Mitchell, M. (2014). Active learning in the atmospheric science classroom and beyond through high-altitude ballooning. J. Coll. Sci. Teach. 44, 26-30.

Cortesão, M., Haas, A. D., Unterbusch, R., Fujimori, A., Schuetze, T., Meyer, V. et al. (2020a). Aspergillus niger spores are highly resistant to space radiation. Front. Microbiol. 11:560. doi: 10.3389/fmicb.2020.00560

Cortesão, M., Schütze, T., Marx, R., Moeller, R., and Meyer, V. (2020b). "Fungal biotechnology in space: why and how?," in Grand Challenges in Fungal Biotechnology, ed. H. Nevalainen (New York, NY: Springer), 501-535. doi 10.1007/978-3-030-29541-7 18

COSPAR (2020). COSPAR Policy on Planetary Protection. Space Res Today 208. Available online at: https://cosparhq.cnes.fr/assets/uploads/2020/07/ PPPolicyJune-2020_Final_Web.pdf (accessed December $8^{\text {th }}, 2020$ ).
Cox, C. S. (1993). Roles of water molecules in bacteria and viruses. Orig. Life Evol. Biosph. 23, 29-36. doi: 10.1007/bf01581988

Cui, B., Smooker, P. M., Rouch, D. A., Daley, A. J., and Deighton, M. A. (2013). Differences between two clinical Staphylococcus ca subspecies as revealed by biofilm, antibiotic resistance, and pulsed-field gel electrophoresis profiling. J. Clin. Microbiol. 51, 9-14. doi: 10.1128/jcm.05124-11

DasSarma, S., DasSarma, P., Laye, V. J., and Schwieterman, E. W. (2020). Extremophilic models for astrobiology: haloarchaeal survival strategies and pigments for remote sensing. Extremophiles 24, 31-41. doi: 10.1007/s00792019-01126-3

de Silva, G. D., Kantzanou, M., Justice, A., Massey, R. C., Wilkinson, A. R., Day, N. P., et al. (2002). The ica operon and biofilm production in coagulase-negative Staphylococci associated with carriage and disease in a neonatal intensive care unit. J. Clin. Microbiol. 40, 382-388. doi: $10.1128 / \mathrm{jcm} .40 .02 .382-38$ 8.2002

Díez, J., Moreno, G., Del Peral, L., Adams, J. H. Jr., Rodríguez Frías, M. D. and Manjón, J. L. (2020). Fuligo septica spores onboard a stratospheric NASA balloon and Its Complete In Vitro Life Cycle. Astrobiology 20, 394-404. doi: $10.1089 /$ ast.2019.2097

Dose, K., Bieger-Dose, A., Ernst, B., Feister, U., Gómez-Silva, B., Klein, A., et al. (2001). Survival of microorganisms under the extreme conditions of the Atacama desert. Orig. Life Evol. Biosph. 31, 287-303.

Dose, K., and Gill, M. (1995). DNA stability and survival of Bacillus subtilis spores in extreme dryness. Orig. Life Evol. Biosph. 25, 277-293. doi: 10.1007/ bf01581591

Eder, W., Schmidt, M., Koch, M., Garbe-Schonberg, D., and Huber, R. (2002). Prokaryotic phylogenetic diversity and corresponding geochemical data of the brine-seawater interface of the Shaban Deep. Red Sea. Environ. Microbiol. 4 758-763. doi: 10.1046/j.1462-2920.2002.00351.x

ESA (2020). ExoMars Mission (2022). Available online at: https://exploration.esa. int/web/mars/-/48088-mission-overview. (Accessed December $8^{\text {th }}, 2020$ ).

Esbelin, J., Mallea, S., Ram, A. F., and Carlin, F. (2013). Role of pigmentation in protecting Aspergillus niger conidiospores against pulsed light radiation. Photochem. Photobiol. 89, 758-761. doi: 10.1111/php.12037

Fairén, A. G., Davila, A. F., Lim, D., Bramall, N., Bonaccorsi, R., Zavaleta, J. et al. (2010). Astrobiology through the ages of Mars: the study of terrestria analogues to understand the habitability of Mars. Astrobiology 10, 821-843. doi: 10.1089 /ast.2009.0440

Fiedler, M. R., Lorenz, A., Nitsche, B. M., van den Hondel, C. A., Ram, A. F., and Meyer, V. (2014). The capacity of Aspergillus niger to sense and respond to cel wall stress requires at least three transcription factors: RlmA, MsnA and CrzA. Fungal Biol. Biotechnol. 1, 5-5.

Fjeldbo, G., and Eshleman, V. R. (1968). The atmosphere of Mars analyzed by integral inversion of the Mariner IV occultation data. Planet. Space Sci. 16, 1035-1059. doi: 10.1016/0032-0633(68)90020-2

Fletcher, L. E., Valdivia-Silva, J. E., Perez-Montano, S., Condori-Apaza, R. M. Conley, C. A., and McKay, C. P. (2012). Variability of organic material in surface horizons of the hyper-arid Mars-like soils of the Atacama Desert. Adv. Space Res. 49, 271-279. doi: 10.1016/j.asr.2011.10.001

Glasheen, J., and Hand, S. (1988). Anhydrobiosis in embryos of the brine shrimp Artemia: characterization of metabolic arrest during reductions in cell-associated water. J. Exp. Biol. 135, 363-380.

Godar, D. E., Wengraitis, S. P., Shreffler, J., and Sliney, D. H. (2001). UV Doses of Americans . Photochem. Photobiol. 73, 621-629. doi: 10.1562/0031-8655(2001) 0730621udoa2.0.co2

Hassler, D. M., Zeitlin, C., Wimmer-Schweingruber, R. F., Ehresmann, B., Rafkin, S., Eigenbrode, J. L., et al. (2014). Mars' surface radiation environment measured with the Mars Science Laboratory's Curiosity rover. Science 343: 1244797

Heldmann, J., Pollard, W., McKay, C., Marinova, M., Davila, A., Williams, K., et al. (2013). The high elevation Dry Valleys in Antarctica as analog sites for subsurface ice on Mars. Planet. Space Sci. 85, 53-58. doi: 10.1016/j.pss.2013.05. 019

Hellweg, C. E., and Baumstark-Khan, C. (2007). Getting ready for the manned mission to Mars: the astronauts' risk from space radiation. Naturwissenschaften 94, 517-526. doi: 10.1007/s00114-006-0204-0

Horneck, G., Klaus, D. M., and Mancinelli, R. L. (2010). Space microbiology. Microbiol. Mol. Biol. Rev. 74, 121-156. 
Jensen, L. L., Merrison, J., Hansen, A. A., Mikkelsen, K. A., Kristoffersen, T., Nornberg, P., et al. (2008). A facility for long-term Mars simulation experiments: the Mars Environmental Simulation Chamber (MESCH) Astrobiology 8, 537-548. doi: 10.1089/ast.2006.0092

Keating, G. M., Pitts, M. C., Brasseur, G., and De Rudder, A. (1987). Response of middle atmosphere to short-term solar ultraviolet variations: 1 . Observations. J. Geophys. Res. Atmos. 92, 889-902. doi: 10.1029/jd092id01p00889

Khodadad, C. L., Wong, G. M., James, L. M., Thakrar, P. J., Lane, M. A., and Catechis, J. A. (2017). Stratosphere conditions inactivate bacterial endospore from a Mars spacecraft assembly facility. Astrobiology 17, 337-350. doi: 10 . 1089/ast.2016.1549

Kuhn, W. R., and Atreya, S. K. (1979). Solar radiation incident on the Martian surface. J. Mol. Evol. 14, 57-64. doi: 10.1007/bf01732367

Lambert, A., Read, W. G., Livesey, N. J., Santee, M. L., Manney, G. L., Froidevaux, L., et al. (2007). Validation of the Aura Microwave Limb Sounder middle atmosphere water vapor and nitrous oxide measurements. J. Geophys. Res. Atmos. 112, 24-36.

Latge, J. P. (1999). Aspergillus fumigatus and aspergillosis. Clin. Microbiol. Rev. 12, $310-350$

Latgé, J.-P., Beauvais, A., and Chamilos, G. (2017). The cell wall of the human fungal pathogen Aspergillus fumigatus: biosynthesis, organization, immune response, and virulence. Annu. Rev. Microbiol. 71, 99-116. doi: 10.1146/ annurev-micro-030117-020406

Leighton, R. B., Murray, B. C., Sharp, R. P., Allen, J. D., and Sloan, R. K. (1965) Mariner IV photography of Mars: initial results. Science 149, 627-630. doi 10.1126/science.149.3684.627

López-Lozano, N. E., Eguiarte, L. E., Bonilla-Rosso, G., García-Oliva, F., MartínezPiedragil, C., Rooks, C., et al. (2012). Bacterial communities and the nitrogen cycle in the gypsum soils of Cuatro Ciénegas Basin, Coahuila: a Mars analogue. Astrobiology 12, 699-709. doi: 10.1089/ast.2012.0840

Mahaffy, P. R., Webster, C. R., Atreya, S. K., Franz, H., Wong, M., Conrad, P. G. et al. (2013). Abundance and Isotopic composition of gases in the martian atmosphere from the curiosity rover. Science 341, 263-266.

Marlow, J. J., Martins, Z., and Sephton, M. A. (2008). Mars on Earth: soil analogues for future Mars missions. Astron. Geophys. 49, 2-20.

Martínez, G. M., Newman, C. N., De Vicente-Retortillo, A., Fischer, E., Renno, N. O., Richardson, M. I., et al. (2017). The modern near-surface martian climate: a review of in-situ meteorological data from viking to curiosity. Space Sci. Rev. 212, 295-338. doi: 10.1007/s11214-017-0360-x

Mertens, C. J. (2016). Overview of the radiation dosimetry experiment (RaD-X) flight mission. Space Weather 14, 921-934. doi: 10.1002/2016sw001399

Mertens, C. J., Gronoff, G. P., Norman, R. B., Hayes, B. M., Lusby, T. C., Straume, T., et al. (2016). Cosmic radiation dose measurements from the RaD-X flight campaign. Space Weather 14, 874-898. doi: 10.1002/2016sw001407

Meyer, V., Wanka, F., van Gent, J., Arentshorst, M., van den Hondel, C. A. M. J. J., and Ram, A. F. J. (2011). Fungal gene expression on demand: an inducible, tunable, and metabolism-independent expression system for Aspergillus niger. Appl. Environ. Microbiol. 77, 2975-2983. doi: 10.1128/aem.02740-10

Moissl-Eichinger, C., Cockell, C., and Rettberg, P. (2016). Venturing into new realms? Microorganisms in space. FEMS Microbiol. Rev. 40, 722-737. doi 10.1093/femsre/fuw015

Mora, M., Wink, L., Kogler, I., Mahnert, A., Rettberg, P., Schwendner, P., et al (2019). Space Station conditions are selective but do not alter microbial characteristics relevant to human health. Nat. Commun. 10:3990

Motamedi, K., Colin, A., Hooijschuur, J., Postma, O., Lootens, R., Pruijser, D., et al. (2015). Design of a Mars atmosphere simulation chamber and testing a Raman Laser Spectrometer (RLS) under conditions pertinent to Mars rover missions. EPJ Tech. Instrum. 2:15.

Murcray, D. G., Kyle, T. G., Kosters, J. J., and Gast, P. R. (1969). The measuremen of the solar constant from high altitude balloons. Tellus 21, 620-624. doi: 10.1111/j.2153-3490.1969.tb00467.x

Musilova, M., Wright, G., Ward, J. M., and Dartnell, L. R. (2015). Isolation of radiation-resistant bacteria from Mars analog Antarctic Dry Valleys by preselection, and the correlation between radiation and desiccation resistance. Astrobiology 15, 1076-1090. doi: 10.1089/ast.2014.1278

NASA (2020). FY 2021 Volume of Integrated Performance). Available online at: https://www.nasa.gov/sites/default/files/atoms/files/fy2021_volume_of integrated_performance.pdf. (Accessed December $8^{\text {th }}, 2020$ )
Novikova, N., De Boever, P., Poddubko, S., Deshevaya, E., Polikarpov, N., Rakova, N., et al. (2006). Survey of environmental biocontamination on board the International Space Station. Res. Microbiol. 157, 5-12. doi: 10.1016/j.resmic. 2005.07.010

Potemra, T. A., and Zmuda, A. J. (1970). Precipitating energetic electrons as an ionization source in the midlatitude nighttime D region. J. Geophys Res. (1896-1977) 75, 7161-7167. doi: 10.1029/ja075i034p07161

Potts, M. (1994). Desiccation tolerance of prokaryotes. Microbiol. Rev. 58, 755-805. doi: $10.1128 / \mathrm{mmbr}$.58.4.755-805.1994

Pulschen, A. A., de Araujo, G. G., de Carvalho, A. C. S. R., Cerini, M. F., de Mendonça Fonseca, L., Galante, D., et al. (2018). Survival of extremophilic yeasts in the stratospheric environment during balloon flights and in laboratory simulations. Appl. Environ. Microbiol. 84, e1942-18.

Rabbow, E., Horneck, G., Rettberg, P., Schott, J.-U., Panitz, C., L'Afflitto, A., et al (2009). EXPOSE, an astrobiological exposure facility on the international space station-from proposal to flight Orig. Life Evol. Biosph. 39, 581-598. doi: 10 . 1007/s11084-009-9173-6

Rabbow, E., Rettberg, P., Barczyk, S., Bohmeier, M., Parpart, A., Panitz, C., et al (2012). EXPOSE-E: an ESA astrobiology mission 1.5 years in space. Astrobiology 12, 374-386. doi: 10.1089/ast.2011.0760

Rabbow, E., Rettberg, P., Barczyk, S., Bohmeier, M., Parpart, A., Panitz, C., et al. (2015). The astrobiological mission EXPOSE-R on board of the International Space Station. Astrobiology 14, 3-16. doi: 10.1017/s1473550414000202

Rainwater, E. L., and Smith, M. S. (2004). Ultra high altitude balloons for mediumto-large payloads. Next Gener. Sci. Ballooning 33, 1648-1652. doi: 10.1016/j.asr. 2003.07.037

Regener, E., and Pfotzer, G. (1935). Vertical intensity of cosmic rays by threefold coincidences in the stratosphere. Nature 136, 718-719. doi: 10.1038/136718a0

Rettberg, P., Rabbow, E., Panitz, C., and Horneck, G. (2004). Biological space experiments for the simulation of Martian conditions: UV radiation and Martian soil analogues. Adv. Space Res. 33, 1294-1301. doi: 10.1016/j.asr.2003. 09.050

Rothschild, L. J., and Mancinelli, R. L. (2001). Life in extreme environments. Nature 409, 1092-1101.

Saganti, P. B., Cucinotta, F. A., Wilson, J. W., Simonsen, L. C., and Zeitlin, C. (2004). "Radiation climate map for analyzing risks to astronauts on the Mars surface from galactic cosmic rays," in 2001 Mars Odyssey, ed. C. Russell (New York, NY: Springer), 143-156. doi: 10.1007/978-0-306-48600-5_5

Schuerger, A. C., Mancinelli, R. L., Kern, R. G., Rothschild, L. J., and McKay, C. P. (2003). Survival of endospores of Bacillus subtilis on spacecraft surfaces under simulated martian environments: implications for the forward contamination of Mars. Icarus 165, 253-276. doi: 10.1016/s0019-1035(03)00200-8

Schuerger, A. C., Trigwell, S., and Calle, C. I. (2008). Use of non-thermal atmospheric plasmas to reduce the viability of Bacillus subtilis on spacecraft surfaces. Astrobiology 7, 47-57. doi: 10.1017/s1473550407 004016

Seele, C., and Hartogh, P. (1999). Water vapor of the polar middle atmosphere annual variation and summer mesosphere conditions as observed by groundbased microwave spectroscopy. Geophys. Res. Lett. 26, 1517-1520. doi: 10.1029/ 1999gl900315

Segers, F. J. J., Wosten, H. A. B., and Dijksterhuis, J. (2018). Aspergillus niger mutants affected in conidial pigmentation do not have an increased susceptibility to water stress during growth at low water activity. Lett. Appl. Microbiol. 66, 238-243. doi: 10.1111/lam.12846

Shepherd, T. G. (2000). The middle atmosphere. J. Atmos. Sol. Terr. Phys. 62, 1587-1601

Silverman, G. J., Davis, N. S., and Beecher, N. (1967). Resistivity of spores to ultraviolet and gamma radiation while exposed to ultrahigh vacuum or at atmospheric pressure. Appl. Microbiol. 15, 510-515. doi: 10.1128/aem.15.3.510515.1967

Simons, D. G. (1954). Methods and results of one year of balloon flights with biological specimens. J. Av. Med. 25, 380-387.

Simonsen, L. C., Nealy, J. E., Townsend, L. W., and Wilson, J. W. (1990). Radiation Exposure for Manned Mars surface missions. NASA Technical Report. Available online at: https://ntrs.nasa.gov/search.jsp?R=19900009041. (accessed December $8^{\text {th }}, 2020$ )

Singaravelan, N., Grishkan, I., Beharav, A., Wakamatsu, K., Ito, S., and Nevo E. (2008). Adaptive melanin response of the soil fungus Aspergillus niger to 
UV Radiation stress at "Evolution Canyon", Mount Carmel, Israel. PLoS One 3:e2993. doi: 10.1371/journal.pone.0002993

Smith, D. J., and Sowa, M. B. (2017). Ballooning for Biologists: Mission essential for flying life science experiments to near space. Gravit. Space Res. 5, 52-73. doi: $10.2478 /$ gsr-2017-0005

Smith, D. J., Thakrar, P. J., Bharrat, A. E., Dokos, A. G., Kinney, T. L., James, L. M., et al. (2014). A balloon-based payload for exposing microorganisms in the stratosphere (E-MIST). Gravit. Space Res. 2, 70-80.

Sobisch, L.-Y., Rogowski, K. M., Fuchs, J., Schmieder, W., Vaishampayan, A., Oles, P., et al. (2019). Biofilm forming antibiotic resistant gram-positive pathogen isolated from surfaces on the International Space Station. Front. Microbiol. 10:543. doi: 10.3389/fmicb. 2019.00543

Stevens, A. W. (1936). Man's farthest aloft Nat. Geogr. 69, 59-94.

Suedfeld, P. (2010). Historical space psychology: early terrestrial explorations a Mars analogues. Planet. Space Sci. 58, 639-645. doi: 10.1016/j.pss.2009.05.010

Sullivan, W. N., and Smith, C. N. (1960). Exposure of house flies and orienta rat fleas on a high-altitude balloon flight. J. Econ. Entomol. 53, 247-248. doi 10.1093/jee/53.2.247

Toner, J., and Catling, D. (2016). Water activities of $\mathrm{NaClO} 4, \mathrm{Ca}(\mathrm{ClO} 4) 2$, and $\mathrm{Mg}$ (ClO4) 2 brines from experimental heat capacities: water activity $>0.6$ below 200 K. Geochim. Cosmochim. Acta 181, 164-174. doi: 10.1016/j.gca.2016.03.005

Vampola, A. L., and Gorney, D. J. (1983). Electron energy deposition in the middle atmosphere. J. Geophys. Res. Space Phys. 88, 6267-6274. doi: 10.1029/ ja088ia08p06267

Von Engeln, A., Langen, J., Wehr, T., Bühler, S., and Künzi, K. (1998). Retrieval of upper stratospheric and mesospheric temperature profiles from Millimeter-Wave Atmospheric Sounder data. J. Geophys. Res. Atmos. 103, 31735-31748. doi: 10.1029/1998jd200035

Vriezen, J. A., De Bruijn, F. J., and Nüsslein, K. (2007). Responses of rhizobia to desiccation in relation to osmotic stress, oxygen, and temperature. Appl. Environ. Microbiol. 73, 3451-3459. doi: 10.1128/aem.02991-06
Wadsworth, J, and Cockell, C. S. (2017). Perchlorates on Mars enhance the bacteriocidal effects of UV light. Sci. Rep. 7:4662.

West, M. D., Clarke, J. D. A., Laing, J. H., Willson, D., Waldie, J. M. A., Murphy, G. M., et al. (2010). Testing technologies and strategies for exploration in Australian Mars analogues: a review. Planet. Space Sci. 58, 658-670. doi: 10. 1016/j.pss.2009.09.022

Winckler, J. R. (1960). Balloon study of high-altitude radiations during the international geophysical year. J. Geophysl. Res. (1896-1977) 65, 1331-1359. doi: 10.1029/jz065i005p01331

Winckler, J. R., Peterson, L., Hoffman, R., and Arnoldy, R. (1959). Auroral $\mathrm{x}$-rays, cosmic rays, and related phenomena during the storm of February 10 11, 1958. J. Geophys. Res. (1896-1977) 64, 597-610. doi: 10.1029/jz064i006p 00597

Wright, J. C. (1989). Desiccation tolerance and water-retentive mechanisms in tardigrades. J. Exp. Biol. 142, 267-292.

Xiao, S., Venkateswaran, K., and Jianga, S. (2019). The risk of Staphylococcus skin infection during space travel and mitigation strategies. Microb. Risk Anal. 11 23-30. doi: 10.1016/j.mran.2018.08.001

Conflict of Interest: The authors declare that the research was conducted in the absence of any commercial or financial relationships that could be construed as a potential conflict of interest.

Copyright (๑ 2021 Cortesão, Siems, Koch, Beblo-Vranesevic, Rabbow, Berger, Lane, James, Johnson, Waters, Verma, Smith and Moeller. This is an open-access article distributed under the terms of the Creative Commons Attribution License (CC BY). The use, distribution or reproduction in other forums is permitted, provided the original author(s) and the copyright owner(s) are credited and that the original publication in this journal is cited, in accordance with accepted academic practice. No use, distribution or reproduction is permitted which does not comply with these terms. 


\section{frontiers}

\section{Supplementary Material}

\section{Supplementary Figures}

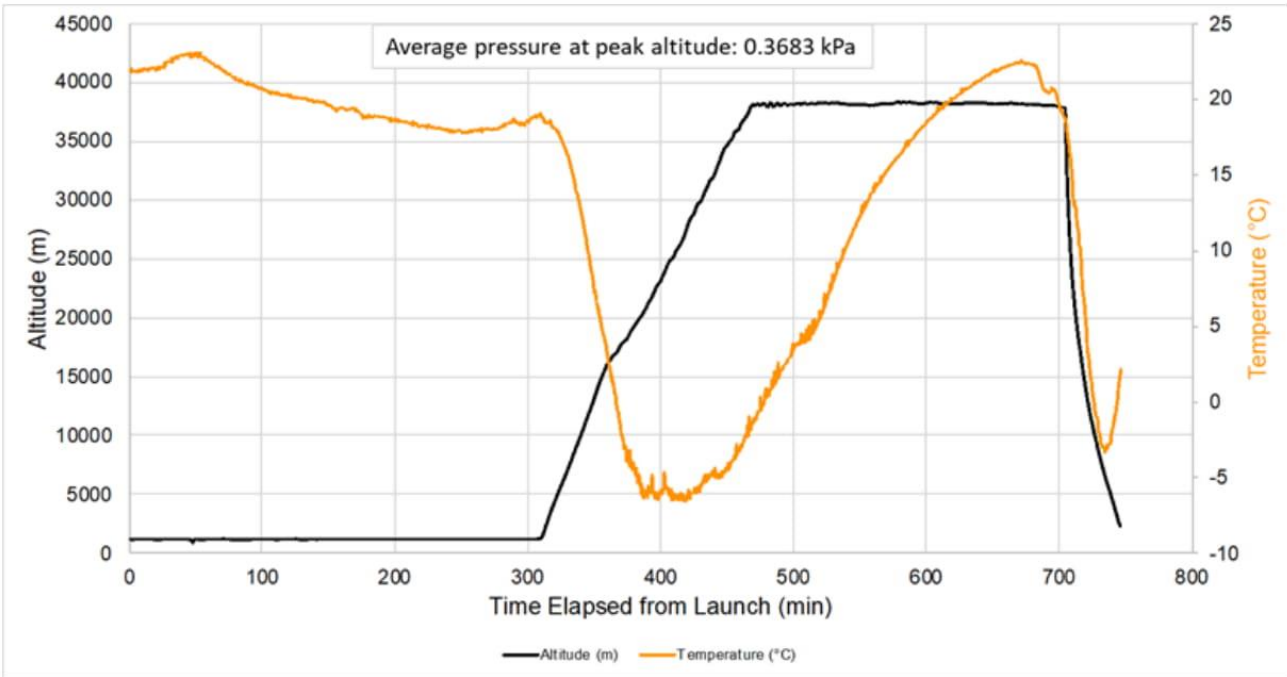

Supplementary Figure 1. Environmental data from the flight via CSBF (outside temperature and pressure and altitude). 


\section{Supplementary Tables}

Supplemental Table 1. Student's t-test was used to identify significant differences between two tested conditions of the survival data using raw data $(\mathrm{CFU} / \mathrm{mL})$. Two-tailed $\mathrm{p}$-value is presented. Equal variances assumed.

$\begin{array}{lllll}\begin{array}{l}\text { t-test } \\ \text { Raw data }\end{array} & \begin{array}{l}\text { Staphylococcus } \\ \text { capitis subsp. } \\ \text { capitis }\end{array} & \begin{array}{l}\text { Salinsisphaera } \\ \text { shabanensis }\end{array} & \begin{array}{l}\text { Aspergillus niger } \\ \text { (monolayer) }\end{array} & \begin{array}{l}\text { Aspergillus niger } \\ \text { (multilayer) }\end{array} \\ \begin{array}{l}\text { Control vs. } \\ \text { UV-shielded }\end{array} & 0.006^{*} & 0.240^{\mathrm{a}} & 0.592 & 0.229^{\mathrm{a}} \\ \begin{array}{l}\text { Control vs. } \\ \text { UV-exposed }\end{array} & 1.000^{\mathrm{a}} & 0.223 & 0.012^{\mathrm{a}} * & 0.016^{\mathrm{a}} * \\ \begin{array}{l}\text { UV-shielded vs. } \\ \text { UV-exposed }\end{array} & 0.006^{*} & 0.136 & 0.012^{\mathrm{a}} * & 0.012^{\mathrm{a}} *\end{array}$

( ${ }^{\text {a) }}$ Mann-Whitney test was used for non-Gaussian distributed data.

(*) $\mathrm{p}<0.05$ was considered significant

Supplemental Table 2. Student's t-test was used to identify significant differences between two tested conditions of the survival data (Survival Fraction N/N0) using mean values and standard error. Two-tailed p-value is presented. Equal variances assumed.

$\begin{array}{lllll}\text { t-test } & \begin{array}{l}\text { Staphylococcus } \\ \text { capitis subsp. } \\ \text { Mapitis }\end{array} & \begin{array}{l}\text { Salinsisphaera } \\ \text { shabanensis }\end{array} & \begin{array}{l}\text { Aspergillus niger } \\ \text { Error }\end{array} & \begin{array}{l}\text { Aspergillus niger } \\ \text { (multilayer) }\end{array} \\ \begin{array}{l}\text { Control vs. } \\ \text { UV-shielded }\end{array} & 0.003^{\mathrm{a} *} & 0.725 & 0.592 & 0.495 \\ \begin{array}{l}\text { Control vs. } \\ \text { UV-exposed }\end{array} & 1.000 & 0.602 & 0.001^{\mathrm{a} *} & 0.053^{*} \\ \begin{array}{l}\text { UV-shielded vs. } \\ \text { UV-exposed }\end{array} & 0.003^{\mathrm{a} *} & 0.513 & 0.001^{\mathrm{a} *} & 0.001^{\mathrm{a} *}\end{array}$

( $\left.{ }^{a}\right)$ Mann-Whitney test was used for non-Gaussian distributed data.

(*) $\mathrm{p}<0.05$ was considered significant 
Supplemental Table 3. ANOVA on Ranks followed by Kruskal-Wallis and Dunn's test were used to identify significant differences in the survival data $(\mathrm{CFU} / \mathrm{mL})$.

\begin{tabular}{|c|c|c|c|c|}
\hline $\begin{array}{l}\text { ANOVA on Ranks } \\
\text { followed by } \\
\text { Kruskal-Wallis } \\
\text { and Dunn's test }\end{array}$ & $\begin{array}{l}\text { Staphylococcus } \\
\text { capitis subsp. } \\
\text { capitis }\end{array}$ & $\begin{array}{l}\text { Salinsisphaera } \\
\text { shabanensis }\end{array}$ & $\begin{array}{l}\text { Aspergillus } \\
\text { niger } \\
\text { (monolayer) }\end{array}$ & $\begin{array}{l}\text { Aspergillus niger } \\
\text { (multilayer) }\end{array}$ \\
\hline $\begin{array}{l}\text { Control vs. } \\
\text { UV-shielded }\end{array}$ & n.a. & 0.905 & 1,000 & 1.000 \\
\hline $\begin{array}{l}\text { Control vs. } \\
\text { UV-exposed }\end{array}$ & n.a. & 0.154 & $0.047^{*}$ & 0.107 \\
\hline $\begin{array}{l}\text { UV-shielded vs. } \\
\text { UV-exposed }\end{array}$ & n.a. & $0.016^{*}$ & $0.034 *$ & $0.014^{*}$ \\
\hline
\end{tabular}

\section{Supplementary Videos}

Supplementary Video 1. Rotatable shutter opening to start sample exposure to UV radiation, once stratosphere altitudes were reached.

Supplementary Video 2. Rotatable shutter closing to stop sample exposure to UV radiation, in preparation for descent.

Our two Supplemental Videos can be accessed through here:

- Supplemental Video 1: https://tubcloud.tu-berlin.de/s/ERLd2dK3xrFSXSj

- Supplemental Video 2: https://tubcloud.tu-berlin.de/s/zSiMadtQLxaLyLr 


\section{Chapter III: Colony growth and biofilm formation of Aspergillus niger under simulated microgravity}

\section{Publication:}

Cortesao, M.. Holland, G. Laue, M., Schuetze, T., Moeller, R and Meyer, V. Growth and biofilm formation of Aspergillus niger under simulated microgravity, npj Microgravity (submitted: NPJMGRAV-00634)

\section{Author contribution}

Planning: 75 \%, Experiment: $90 \%$, Manuscript: $80 \%$

M.C. designed and performed the experiments and analyzed the data. G.H. and M.L. assisted with SEM experimental design, sample preparation, imaging and analysis. M.C., T.S., V.M. and R.M. designed the study and jointly interpreted the data. M.C. and V.M. co-wrote the manuscript which was approved by all co-authors. 


\title{
Colony growth and biofilm formation of Aspergillus niger under simulated microgravity
}

\author{
Marta Cortesao ${ }^{1,2 *}$, Gudrun Holland ${ }^{3}$, Tabea Schütze ${ }^{2}$, Michael Laue ${ }^{3}$, Ralf Moeller ${ }^{1}$ and Vera \\ Meyer $^{2}$
}

${ }^{1}$ German Aerospace Center (DLR), Institute of Aerospace Medicine, Radiation Biology Department, Aerospace Microbiology Research Group, Cologne, Germany

${ }^{2}$ Chair of Applied and Molecular Microbiology, Institute of Biotechnology, Technische Universität Berlin, Berlin, Germany

${ }^{3}$ Robert Koch Institute, Advanced Light and Electron Microscopy (ZBS 4), Berlin, Germany

* Corresponding author: E-mail: marta.cortesao@dlr.de, Phone +49(2203) 601-4211

\section{ABSTRACT}

Knowing how spaceflight microgravity affects Aspergillus niger's ability to grow and form biofilms is not only crucial to ensure astronaut health and spacecraft safety but it will also pave the way for exploitation of fungal-based biotechnology during space exploration. We thus analyzed colony growth and biofilm formation of three $A$. niger strains under simulated microgravity: a wild-type, a pigmentation mutant defective in melanin formation, and a hyperbranching mutant of biotechnological interest. Sample preparation for scanning electron microscopy (SEM) was able to preserve the ultrastructure of $A$. niger colonies generating high resolution images of $A$. niger's mycelial network, extracellular matrix, conidiophores and spores. Notably, simulated microgravity led to denser biofilms in the wild-type and the hyperbranching mutant and increased spore production in both the pigmentation and the hyperbranching mutant. This suggests that colony and biofilm formation of $A$. niger is affected by spaceflight conditions in a strain-dependent manner.

Keywords: Aspergillus niger, simulated microgravity, biofilm, scanning electron microscopy, pigmentation, conidiation, mycelium network, GTPase, racA, polyketide synthase, fwnA 


\section{INTRODUCTION}

Current crewed spaceflight activities are done in low-Earth orbit (LEO) aboard the International Space Station (ISS). The ISS is an indoor-closed habitat that creates a comfortable environment by shielding those on board from the extremes of space. However, spaceflight microgravity $\left(10^{-6} \mathrm{~g}\right.$ compared to $1 \mathrm{~g}$ on the Earth's surface) inevitably affects all living systems aboard the ISS. Indeed, microgravity is known to induce changes in numerous cellular processes such as nutrient transport, metabolism or signaling ${ }^{1-3}$, and was shown to induce changes in virulence in human pathogenic bacteria like Serratia marcescens, Escherichia coli and Pseudomonas ${ }^{4-7}$. The fungus Aspergillus niger is a common isolate of the ISS's microbiota ${ }^{8}$. On Earth, industrial $A$. niger strains are exploited in biotechnology for the production of proteins, enzymes and organic acids, playing an important role in the transition from a petroleum-based economy into a biobased circular economy $y^{9,10}$. Nevertheless, $A$. niger natural isolates are also known as disease agents of the lung infection aspergillosis in immunocompromised patients ${ }^{11}$. This happens primarily due to $A$. niger's highly resistant and airborne spores (named conidia) that can contaminate, colonize and biodegrade surfaces. These surfaces can be both biotic (e.g. human lungs) and abiotic (e.g. surgical or construction materials, or air- and water systems $)^{12-14}$. Such surface-associated contamination scenarios are found not only in space stations but also in hospitals, industrial facilities and airplane cabins ${ }^{8,15-18}$. For these reasons, and given that $A$. niger spores are highly resistant to space radiation ${ }^{19}$, their uncontrolled presence in spaceflight habitats will be particularly challenging during long-term space missions, i.e., to the Moon, or Mars ${ }^{20,21}$. Despite these threats, a monitored and controlled use of $A$. niger for biotechnological purposes in long-term space missions can be a valuable resource for sustainable space exploration ${ }^{21}$. Thus, knowing how $A$. niger can colonize solid surfaces, how it grows into colonies that resemble biofilms due to the formation of an extracellular matrix ${ }^{22}$ and how its spores are produced under spaceflight microgravity will be critically important to help maintain astronaut health and safety of habitats, on Earth and in space ${ }^{21}$.

Most fungal contaminations and infections occur in static, aerial and surface-associated conditions. Because of that, a mature $A$. niger colony grown on agar is an established model system for fungal biofilmmediated infections in human lungs ${ }^{23-25}$ and can also serve as model for spacecraft surface contaminations. A mature colony is the result of the outgrowth of a single spore that germinated and elongated into long cell threads named hyphae. These hyphae frequently branch and ultimately create a complex interwoven network of hyphae called vegetative mycelium, both on and in the substrate. This mycelium is composed of multinucleated hyphae and is therefore considered a syncytia, i.e. multinucleated cells sharing a common cytoplasmic environment and functioning as a single coordinated unit $^{26}$. Notably, this vegetative mycelium can be embedded in an extracellular matrix (ECM), which is rich in exopolysaccharides such as $\alpha$-glucans, galactomannan and galactosaminogalactan, extracellular DNA, proteins, lipids and polyols ${ }^{27,28}$. When nutrients become limited beneath the vegetative mycelium, aerial hyphae form out of the mycelium to explore the air space. Some of these aerial hyphae differentiate into conidiophores for asexual sporulation (conidiation). The conidiophores produce up to 10,000 spores each and stay high above the vegetative mycelium to release the spores into the environment using the air flow $^{29}$. This facilitates spread and subsequent colonization of other habitats.

Only very few studies have so far addressed the growth and adaptation of $A$. niger and related fungal species to microgravity aboard the ISS ${ }^{30,31}$. As experiments on the ISS are very cost-intensive, difficult to 
implement, and extremely limited in throughput, microgravity simulation methods are valid alternative approaches that can be easily performed on Earth to study adaptation phenomena of filamentous fungi to microgravity. For example, previous studies with submerged liquid cultures of $A$. niger and Penicillium chrysogenum under low-shear modeled microgravity in the High Aspect Ratio Vessel (HARV), at a rotation speed of 25 r.p.m., found no significant changes in spore germination, mycelium growth or cell wall integrity $^{32}$. Similar HARV liquid cultures of $P$. chrysogenum reported transcriptomic changes in cell wall transporters and enzymes involved in penicillin biosynthesis ${ }^{33}$. Notably, the clinostat has been recognized to provide better simulation of microgravity than HARV ${ }^{34,35}$. Growth of the food spoilage fungus $A$. carbonarius in solid, static and aerial conditions under simulated microgravity by slow-clinorotation of 20 r.p.m., revealed no changes in cell growth or colony appearance, but showed an increase in organic acid production (e.g. oxalic acid and citric acid) ${ }^{36}$.

In this study we have investigated $A$. niger colony growth in solid media, static and aerial conditions, under simulated microgravity by fast-clinorotation of 60 r.p.m. Three different strains of the same lineage were tested: an industrial wild-type strain, a strain defective in pigmentation $(\triangle f w n A)$ which is more susceptible to space radiation ${ }^{19}$, and a hyperbranching mutant strain $(\triangle \operatorname{rac} A)$ that is identical to the wild-type strain with respect to biomass formation but produces about $20 \%$ more hyphal tips, and is thus of interest as biotechnological platform strain due to its protein hypersecretion phenotype ${ }^{37}$. We present the impact of simulated microgravity on $A$. niger's colony ultrastructure through scanning electron microscopy (SEM) images of colony cross-sections. We also present data addressing the effect of simulated microgravity on the strains' colony surface area, biomass accumulation, spore production and spore vitality. Finally, we suggest that fwnA-mediated pigmentation is important for $A$. niger's adaptation to simulated microgravity, affecting colony growth, mycelium network organization and spore production.

\section{RESULTS}

Wild-type, pigmentation ( $\triangle f w n A)$ and hyperbranching $(\triangle \operatorname{rac} A)$ mutant strains were grown as biological triplicates in petri-dishes filled with minimal medium agar under normal gravitational conditions on Earth (Ground) and under simulated microgravity (SMG) conditions. Microgravity was simulated via a fastrotating petri-dish clinostat that reaches $1.2 \times 10^{-2}$ to $1.6 \times 10^{-2} \mathrm{~g}$, in a colony radius $(r)$ between $0.3-0.4$ $\mathrm{cm}$ (Figure 1). Colony area, dry biomass and spore production were calculated for 3 -day old colonies under Ground and SMG conditions to study early stages of colonization. In addition, SEM analyses were performed for 5-day old colonies to better distinguish between two different regions - center and edge that correspond to late and early stages of maturation, respectively (Figure 1). The effect of simulated microgravity was only investigated in the central region $(0-0.4 \mathrm{~cm})$, where morphological changes with regards to colony ultrastructure were assessed, including thickness of the vegetative mycelium as well as conidiophore height. In addition, the vitality of spores produced under Ground and SMG conditions was assessed by analyzing their metabolic activity, spore hydrophobicity and germination rate. 


\section{Colony morphology of $\boldsymbol{A}$. niger strains under normal gravitational conditions}

3-day old wild-type colonies reached an area of $3.3 \mathrm{~cm}^{2}$, accumulated a total of $8.7 \mathrm{mg}$ dry biomass and produced on average $6 \times 10^{7}$ spores (Figure 1, Figure 2 and Supplemental Table 1). In comparison, the pigmentation mutant $\triangle f w n A$ produced slightly larger colonies (on average $3.7 \mathrm{~cm}^{2}$ ), accumulated about 30 $\%$ less biomass but produced significantly more spores $\left(9 \times 10^{7}\right.$ spores, $\left.p=0.04\right)$, which were less hydrophobic. In contrast, $\triangle$ racA colonies were significantly smaller than the wild-type colonies (on average $\left.1.2 \mathrm{~cm}^{2}, p=0.02\right)$, but accumulated a similar dry biomass $(9.7 \mathrm{mg})$ as previously described due to its hyperbranching phenotype ${ }^{37}$ and produced significantly less spores $\left(6.8 \times 10^{6}, p<0.01\right)$.

SEM images of whole colony cross-sections from the wild-type strain revealed morphological differences among the different regions of the colony (Figure 3). Cross-sections of the youngest part of the colony (edge region) were populated with foraging hyphae that explore the environment and secrete high amounts of enzymes. These hyphae were connected in a loose network and were filled with cytoplasm. In contrast, hyphae from the oldest part of the colony (central region) were devoid of cytoplasm, with only a few granular remnants likely left due to autophagic processes. This data was confirmed by transmission electron microscopy (TEM), for which we used another fixation method (high-pressure freezing instead of chemical fixation) in order to exclude the possibility that sample fixation could unintentionally alter hyphal morphologies (Figure 3 and Supplemental Figure 1). Autophagy in Aspergillus is known to be related with aging and integral to nutrient and organelles recycling in older parts of a syncytium and is thought to facilitate growth of aerial hyphae and conidiophores ${ }^{38,39}$. Conidiophores emanating from the central region were on average $460 \mu \mathrm{m}$ high (Supplemental Table 1) and displayed morphological characteristics for conidiogenesis as published earlier ${ }^{40}$ : vesicles that developed from stalks formed primary sterigmata (metulae), secondary sterigmata (phialide) and chains of spores which were surrounded with an undulated surface coating consisting of melanin and hydrophobins (Figure 3a, b, g-i and Supplemental Figure 2). Remarkably, wild-type hyphae from the central region (oldest part of the colony) were embedded in extracellular matrix (ECM), resulting in a $60 \mu \mathrm{m}$ thick biofilm, which was not present around the young hyphae at the colony edge (Figure 3c-f). Similarly, hyphae from the central region of the $\triangle$ fwnA mutant were embedded in ECM, resulting in a biofilm thickness of $\sim 70 \mu \mathrm{m}$. Conidiophores of $\triangle$ fwnA mutant reached a height of $\sim 450 \mu \mathrm{m}$, and spores lacked, as expected, the characteristic melanin/hydrophobin layer on the spore surfaces (Figures 4-7 and Supplemental Figure 2). Interestingly, SEM data uncovered that the vegetative mycelium formed in the central region of $\triangle$ racA colonies is about 3-times thicker compared to the wild-type and the $\Delta f w n A$ mutant ( $175 \mu \mathrm{m}$, Figure 4 and Supplemental Figure 4), which would explain similar dry biomass formation compared to the wild-type despite its reduced colony diameter (Figure 2). The structure of the vegetative mycelium in the central region of the $\triangle$ racA mutant also differed from that of the other two strains. While wild-type and $\triangle f w n A$ vegetative mycelia produced an ECM that consisted of two distinct layers: an upper layer with hyphae embedded in dense and porous ECM, and a lower layer with loose hyphae and with an thin ECM that coated only individual hyphae (Figure 5); the vegetative mycelium of the $\triangle r a c A$ mutant consisted out of three layers: an upper layer (63 $\pm 2.7 \mu \mathrm{m})$ with hyphae embedded in a dense ECM, a middle layer $(87 \pm 3.5 \mu \mathrm{m})$ with loose, vertically oriented hyphae not embedded in an ECM, and a lower layer ( $45 \pm 1.6 \mu \mathrm{m})$, also with loose but horizontally oriented hyphae which were not embedded in an ECM (Figure 5). Furthermore, it became evident that the hyperbranching 
$\triangle$ racA mutant formed shorter spore chains on often malformed vesicles, although the spore surface topology itself resembled the topology of wild-type spores (Figure 6 and Supplemental Figures 2-3).

\section{Colony morphology of $A$. niger strains under simulated microgravity}

When all three strains were cultivated under SMG conditions, a slight increase in colony diameter was seen for all tested strains (Figure 2), suggesting that SMG might support colonization of surfaces. Simulated microgravity also affected biomass formation in both mutant strains: the pigmentation mutant produced more biomass whereas the hyperbranching mutant produced less. Remarkably, the number of spores produced under SMG conditions increased significantly for both mutant strains ( $26 \%$ in $\triangle$ fwnA mutant, $p$ $=0.03$, and $35 \%$ in $\Delta r a c A, p=0.03)$, and increased slightly in the wild-type strain $(17 \%, p=0.2)$, implying that asexual sporulation might occur faster under simulated microgravity conditions, but that this is, however, strain-dependent. Conidiophore height, spore hydrophobicity as well as spore morphology and surface topology did not change significantly for either of the three strains under SMG compared to Ground conditions. It can thus be concluded that the integrity of $A$. niger spores is not likely to be affected by the microgravity environment (Figure 2, Figure 6 and Supplemental Table 1).

Furthermore, SEM demonstrated that SMG conditions induced changes in the thickness of the vegetative mycelium in central region of the colonies. The thickness of the vegetative mycelium increased significantly by $22 \%$ in the wild-type ( $p=0.001), 56 \%$ in the hyperbranching mutant $(p=0.04)$, but only slightly and non-significantly in the pigmentation mutant $(6 \%, p=0.1$, Figure 5, and Supplemental Table 1). A closer look at the hyperbranching $\triangle \mathrm{racA}$ mutant showed that, independently of the gravitational regime, it formed a folded vegetative mycelium from which aerial hyphae and conidiophores emanated in both directions, i.e., upward and downward (Supplemental Figure 4). Many of the conidiophore stalks in $\triangle \operatorname{racA}$ were able to bud off spores (Supplemental Figure 2), a phenomenon which we only seldomly observed in the wild-type and $\triangle f w n A$ strains. The three distinct layers of vegetative mycelium of the $\Delta$ racA mutant were also seen in SMG conditions, with no differences in thickness when compared to Ground: upper layer $(59 \pm 1.5 \mu \mathrm{m})$, a middle layer $(90 \pm 5.2 \mu \mathrm{m})$, and lower layer $(45 \pm 2.4 \mu \mathrm{m}$, Figure 5$)$.

\section{Viability of $A$. niger spores under Ground and SMG conditions}

We finally assessed the metabolic activity as well as germination rate of asexual spores harvested from the central colony regions. As summarized in Figure 7 and Supplemental Table 2, the maximum of metabolic activity of germinated spores from all strains was reached after 24 - $25 \mathrm{~h}$ post-inoculation under normal gravitation conditions, and was slightly shifted for the $\triangle$ racA strain, towards $21 \mathrm{~h}$ under SMG. However, no significant microgravity-induced changes in germination rates and metabolic activities were observed. 
a

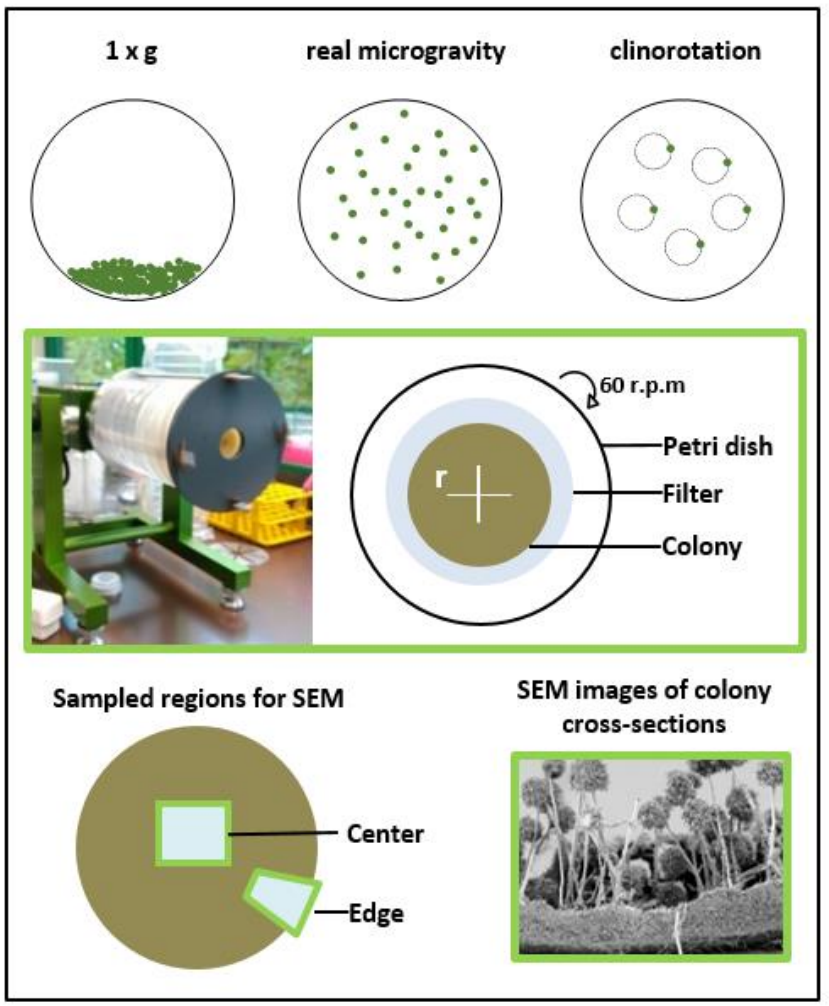

b
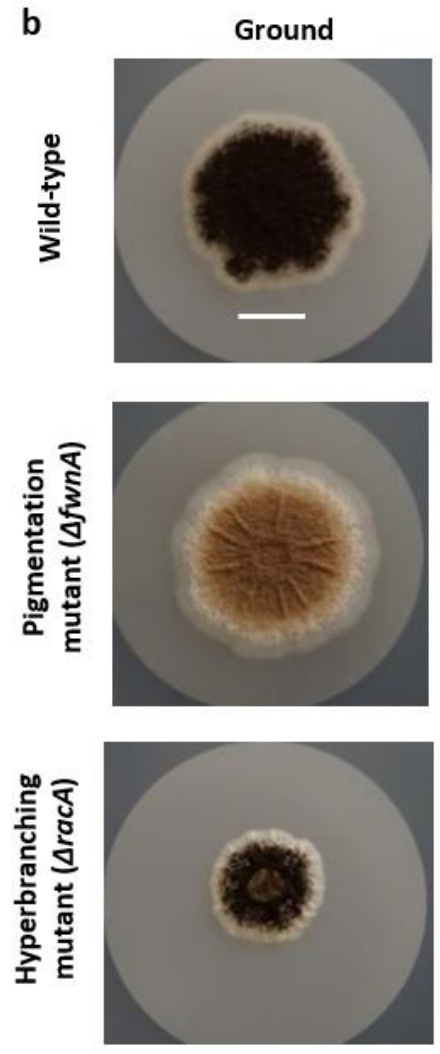

SMG
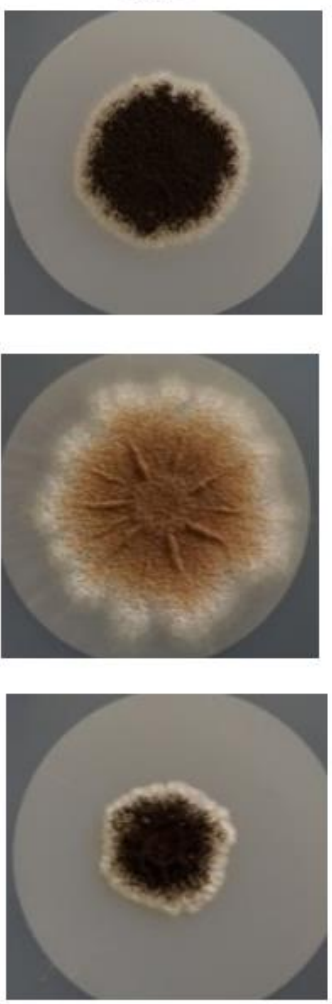

Figure 1. Simulation of microgravity in a petri-dish clinostat and effect of simulated microgravity in colony area. $\boldsymbol{a}$ Colonies were grown on a filter that covered minimal medium agar in Petri-dishes. The plates fit into the clinostat that simulates microgravity by continuously rotating the petri dishes (60 r.p.m.). Here, the central region of the colony can achieve g-forces from $1.2 \times 10^{-2}$ to $1.6 \times 10^{-2} \mathrm{~g}$, in a colony $(\mathrm{r})$ radius between $0.3-0.4 \mathrm{~cm}$. Colonies were sampled for SEM in the center and at the edge. $\mathbf{b}$ Pictures of representative colonies of the three tested strains after 5 days of cultivation (scale $=1 \mathrm{~cm}$ ). 
a

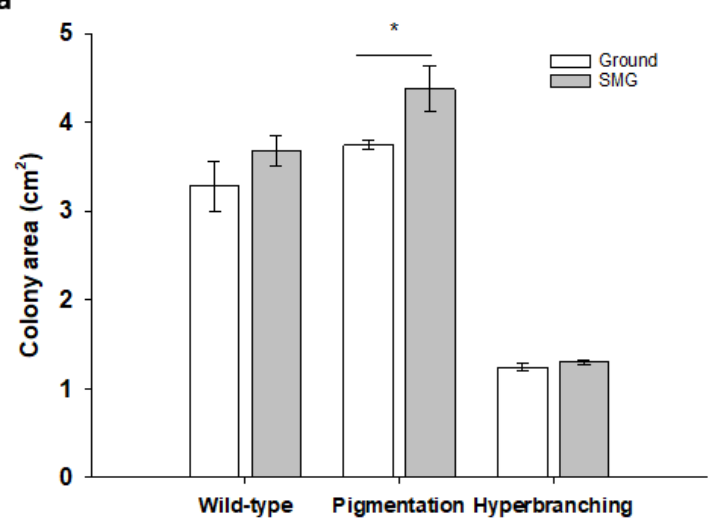

C

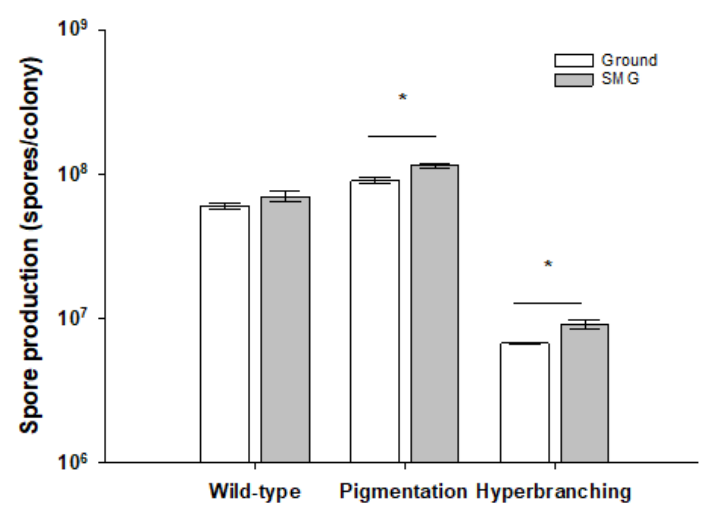

b

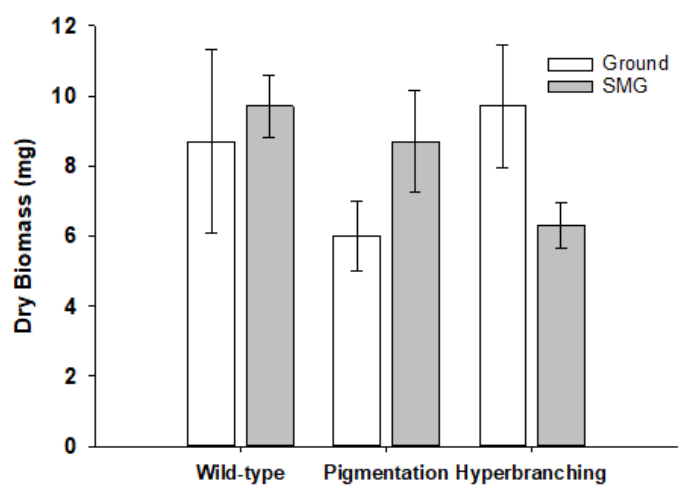

d

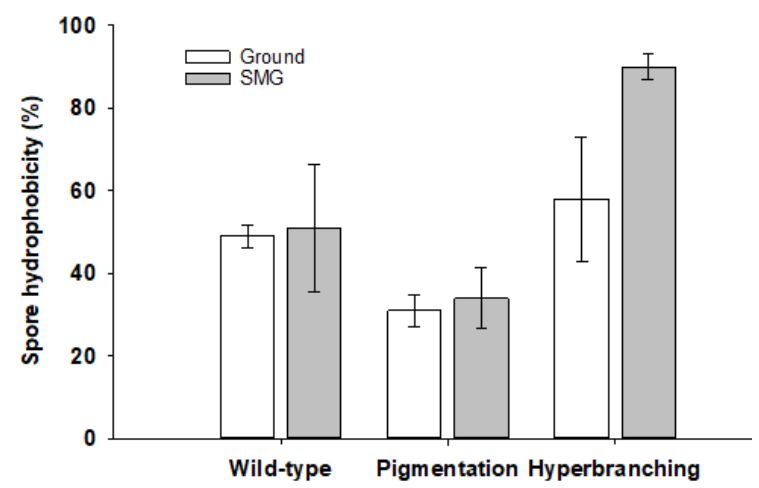

Figure 2. Simulated microgravity induces changes in A. niger colony area, dry biomass area, spore production and spore hydrophobicity in a strain-dependent manner. Spores of the three test strains were used to inoculate minimal medium agar plates as biological triplicates. After three days of incubation, several morphological characteristics were determined. a Colony area. b Dry biomass. c Spore production per colony. d Spore hydrophobicity. Data shown as mean with error bars as standard error, where $p \leq 0.05$ was considered significant and is indicated with an asterisk. 

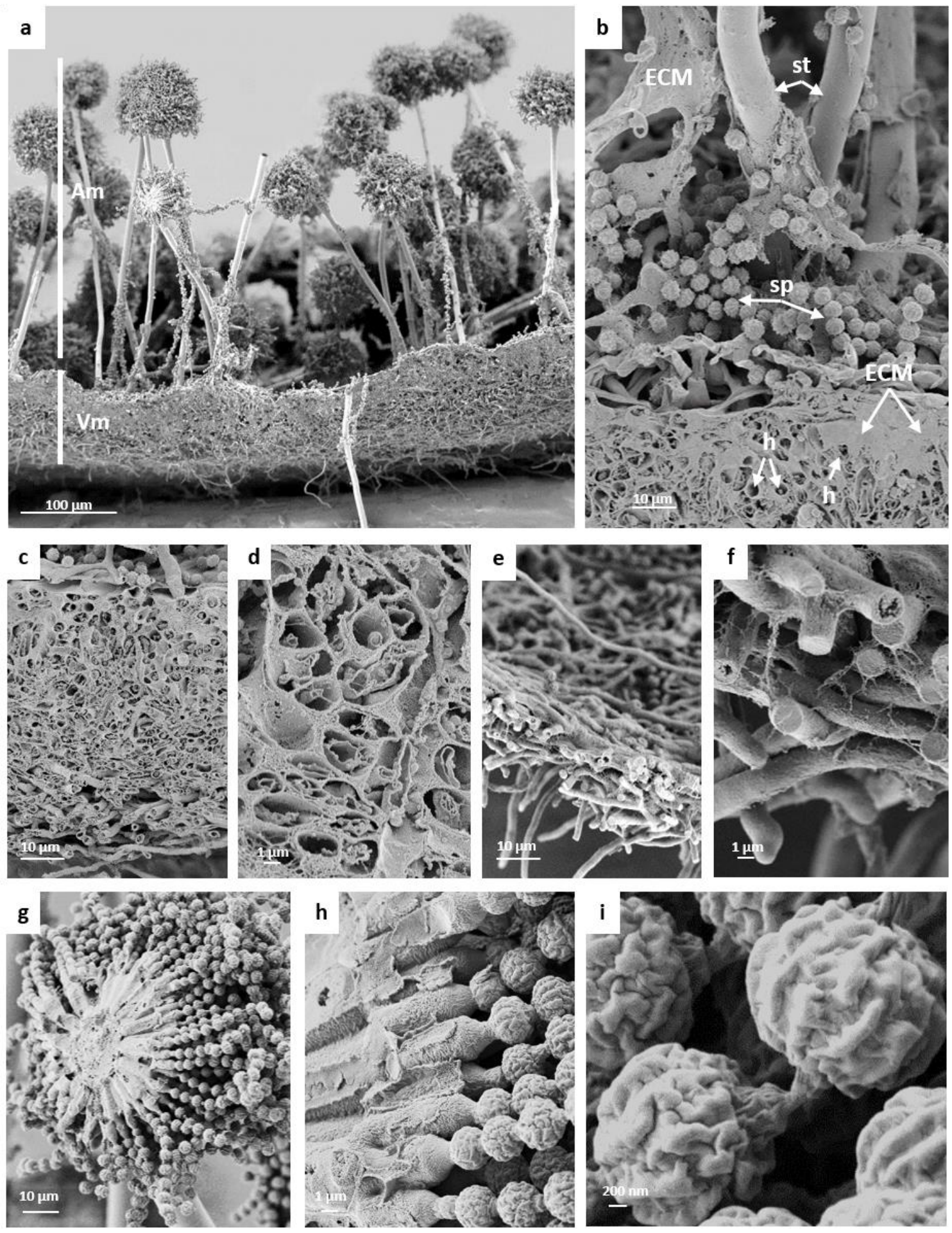

Figure 3. SEM images of Aspergillus niger wild-type colonies grown in static, aerial conditions. a vegetative ( $V m)$ and aerial mycelia (Am) with conidiophores. b close-up view of the interface of aerial and vegetative mycelium showing extracellular matrix (ECM), stalks (st), loose spores (sp) and vegetative hyphae (h). c ECM that covered vegetative mycelium in the center region of the colony. $\mathbf{d}$ Hollow hyphae in the center region. $\mathbf{e}, \mathrm{f}$ hyphae at the edge of the colony not yet covered with ECM. $\mathbf{f}$ close-up view of compact hyphae filled with cytoplasm at the colony edge. $\mathbf{g}$ cross-section of $A$. niger conidiophore revealing stalk, vesicle, metulae, phialides and conidia chains. h,i close-up view of phialides and spore chains, revealing folded outer layer of spores. 

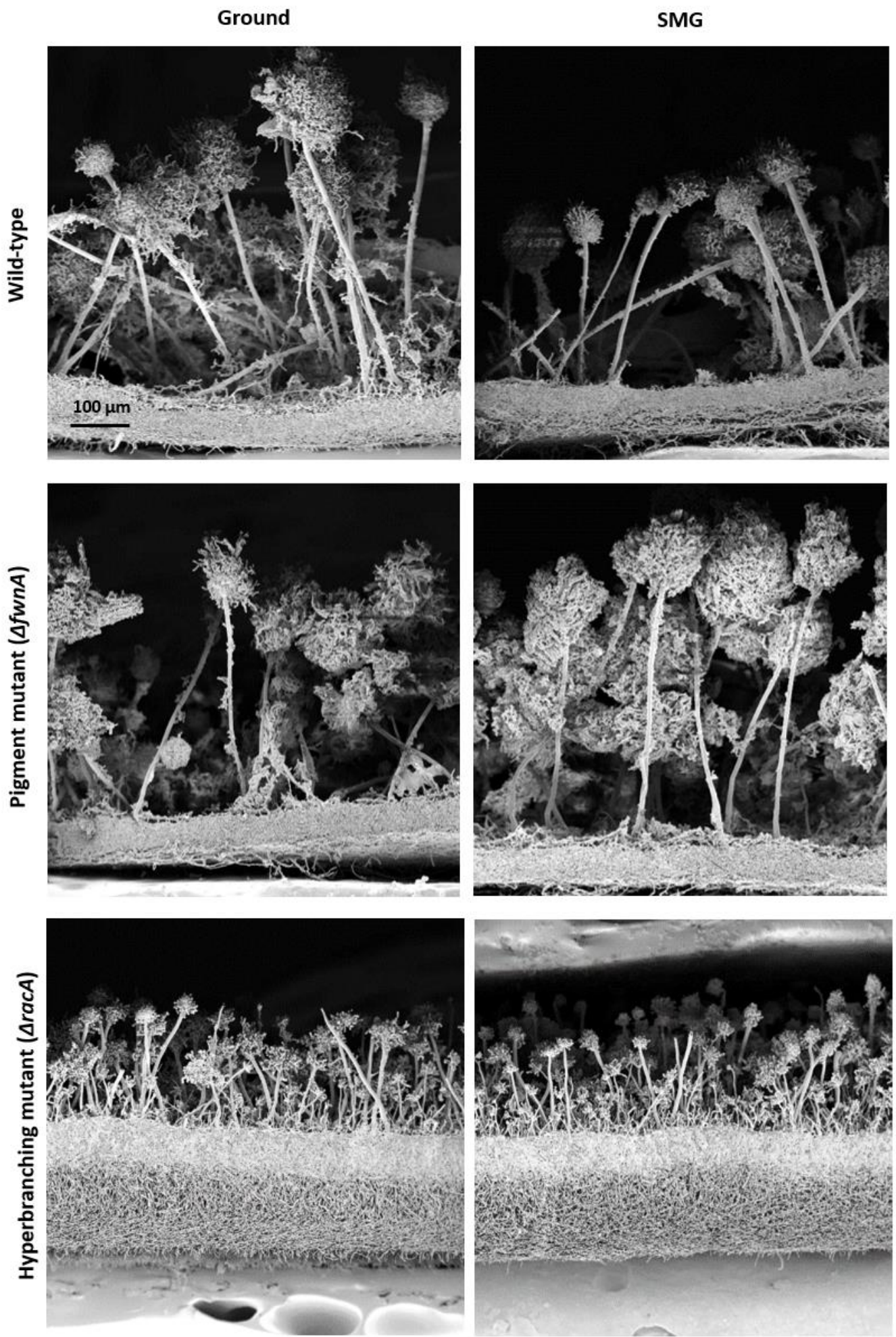

Figure 4. SEM images of A. niger strains from the central region of colonies grown in Ground and simulated microgravity (SMG) conditions. Representative images of colony fragment cross-sections showing differences in vegetative and aerial mycelia for the tested strains. Note that the thickness of the vegetative mycelium and the height of conidiophores differs among the strains. Scale is the same for all images. 

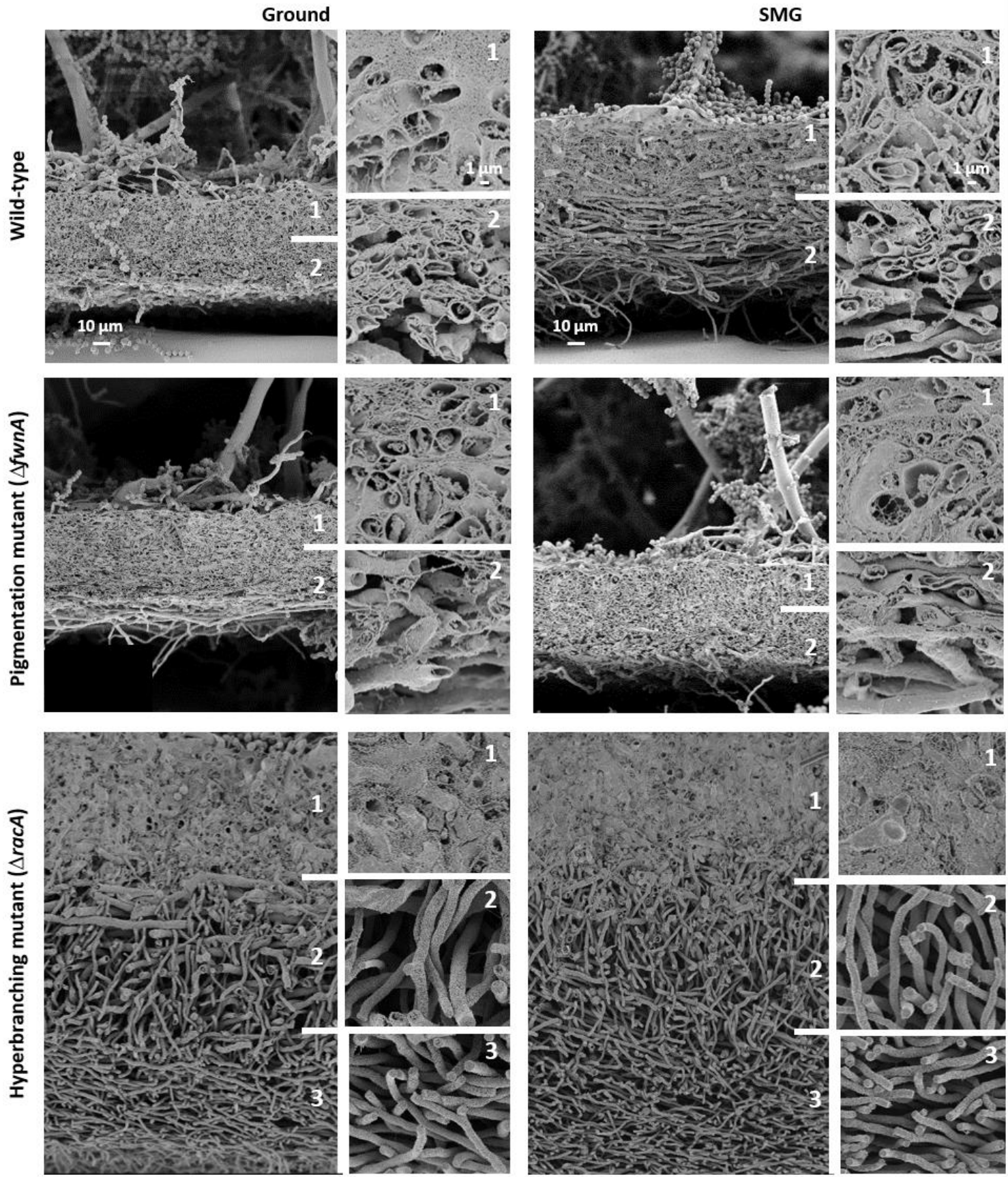

Figure 5. Vegetative mycelium ultrastructure of different A. niger strains grown under Ground and simulated microgravity (SMG) conditions. Note that the thickness of the vegetative mycelium differs between the strains.

Two or three layers of the vegetative mycelium can be distinguished in a strain-dependent manner and are indicated with numbers 1, 2 and 3. Scale is the same for all images in each column. 

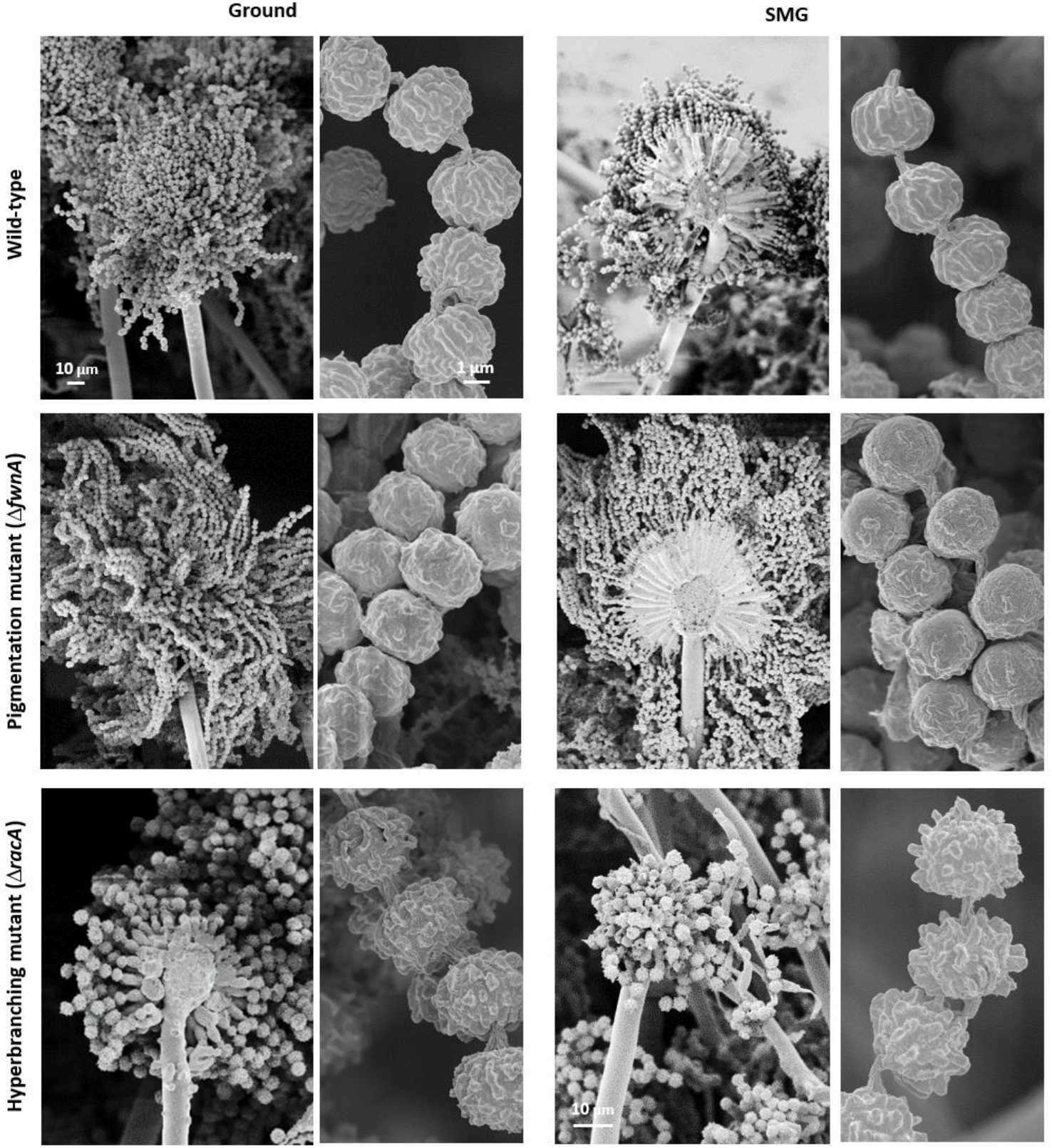

Figure 6. Conidiophores and spore chains of A. niger strains under Ground and simulated microgravity (SMG) conditions. Note that the length of the spore chains differs between strains, which can be up to $150 \mu \mathrm{m}$ in the $\Delta$ fwnA mutant. Scale is the same for all images in each column. 
a

Ground
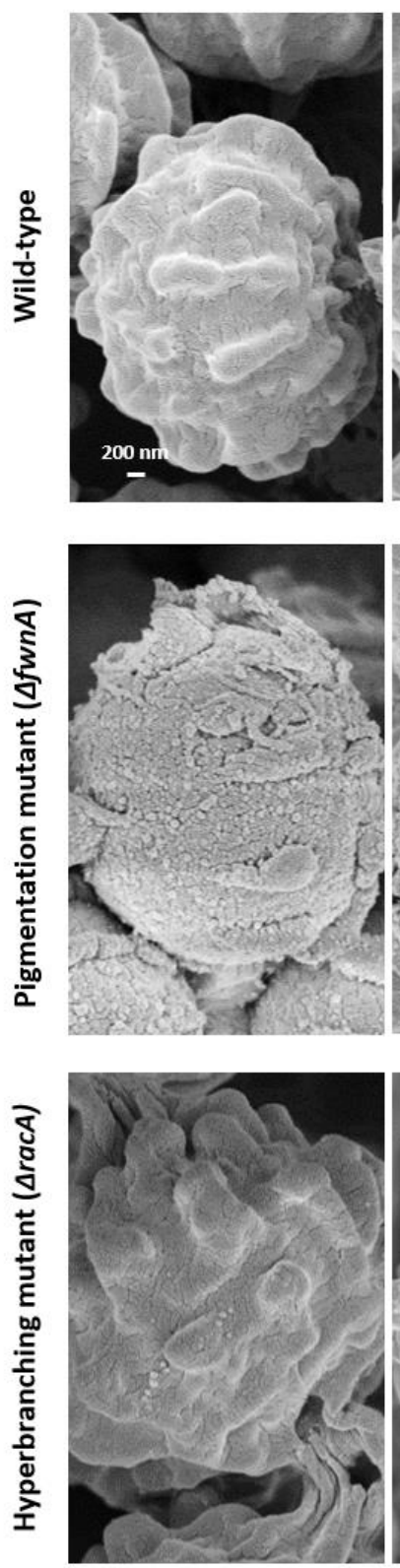

SMG
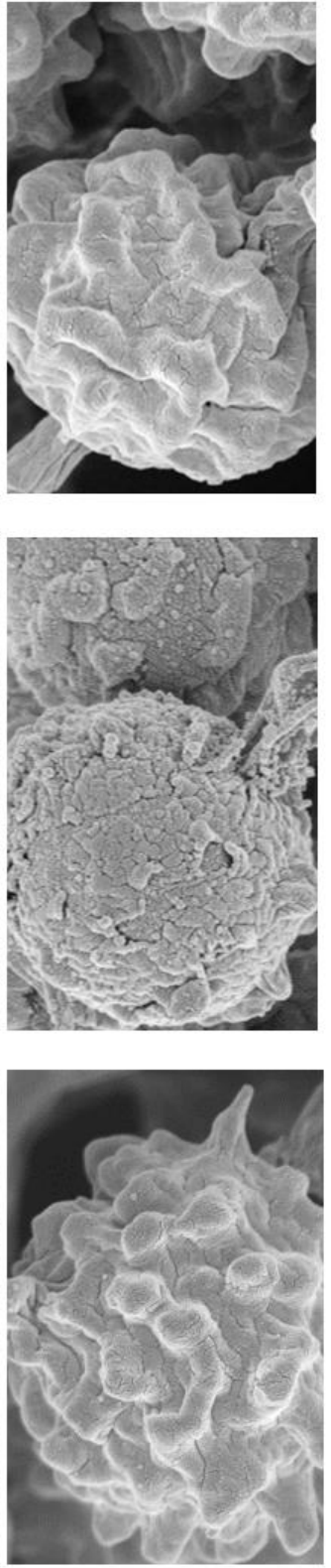

b
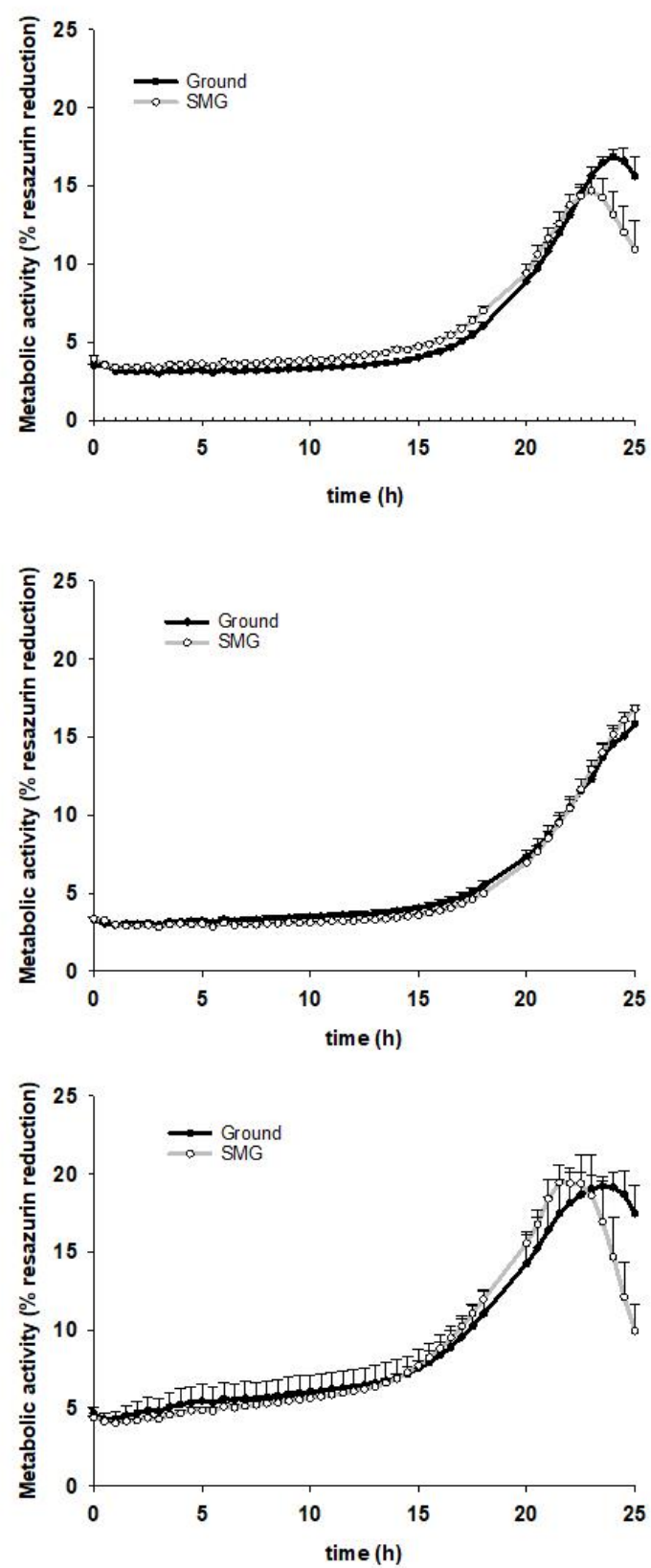

Figure 7. Spore morphology and vitality from A. niger colonies grown under Ground and simulated microgravity (SMG) conditions. a SEM of spores of each tested strain show no morphological differences when produced under SMG. Differences between spore surfaces of wild-type and $\triangle f w n A$ mutant can be seen, as a defect in pigmentation leads to changes in the outer layer of the cell wall. $\mathbf{b}$ metabolism upon germination show no significant changes under SMG. Data shown as mean from biological triplicates with error bars. 


\section{DISCUSSION}

In this study, we performed high-resolution SEM of whole colony cross-sections of the industrially and medically important filamentous fungus $A$. niger. SEM analysis demonstrate the complex and intricate ultrastructure of the mycelium through which $A$. niger explores and colonizes its surrounding environment. Furthermore, our study investigated changes in A. niger colony growth in both Ground and simulated microgravity (SMG) conditions. Changes in A. niger colony growth likely affect surface adhesion and intercellular cohesion, both key factors for contamination potential and decontamination resistance. This is particularly important in indoor-closed environments, such as hospitals or space stations, where monitoring and controlling fungal contaminations is challenging.

SEM of wild-type $A$. niger colonies under normal gravitational conditions showed that hyphae at the colony edge were not significantly covered by an extracellular matrix (ECM), while hyphae in the colony center were at least partially embedded in a dense ECM. This suggests that the colony develops into a biofilm, i.e. ECM embedded vegetative mycelium, during maturation. In a mature colony, the ECM was mainly found in the upper layer of the vegetative mycelium suggesting its role in shielding the colony from environmental stressors including cleaning agents, antimicrobials and fungicides. As we have proposed above, young hyphae of $A$. niger mainly work as nutrient scavengers, having a highly active and compact cytoplasm, whereas old hyphae are important in setting the biofilm's three-dimensional structure (where only the cell wall is needed and thus the cytoplasm appears empty). Previous studies have identified, accordingly, that mature fungal colony biofilms are composed of both active and inactive hyphae ${ }^{29,44}$. SEM showed that the youngest part of the wild-type colony (at the very edge) was composed of a single layer of hyphae with an intact cytoplasm. This early colony stage of hyphae multilayer is similar to what Krijgsheld et al. reported for an A. niger 7-day sandwiched colony ${ }^{29}$. In contrast, hyphae in the center of the colony, which represent the oldest and matured part of the colony, appeared rather empty without any structural signatures indicating intact cytoplasm. This was confirmed by performing TEM of colonies subjected to physical fixation, to discard the possibility of artifacts introduced by chemical fixation (Supplemental Figure 1). A. niger colony growth in static, aerial conditions can be summarized as follows: 1) spore germination and outgrowth followed by hyphal expansion and increase in colony area; 2) hyphae multilayering and increase in vegetative mycelium thickness; 3 ) secretion of ECM and maturation into a biofilm; 4) cell death and transition from active hyphae (nutrient scavenging) to inactive hyphae (structural); 4) formation of aerial hyphae, conidiophores and sporulation. Furthermore, quantitative analysis of the SEM images showed that $A$. niger wild-type conidiophores can reach an average height of $\sim 460 \mu \mathrm{m}$. This is four times higher than initially reported, for example in A. nidulans $(100 \mu \mathrm{m}){ }^{45}$, and suggests an efficient air-dispersal ability of $A$. niger's spores.

Our study shows that simulated microgravity affects $A$. niger colony area, spore production and biofilm thickness in a strain-dependent manner. We propose that RacA might play a role in A. niger's adaptation to simulated microgravity, as its deletion leads to increased mycelium thickness, increased spore production and decreased total biomass during SMG conditions. RacA controls actin polymerization in $A$. niger and is thus responsible for maintaining hyphal tip polarity and hyphal branch initiation ${ }^{43}$ - both are essential for the establishment of the complex hyphal network characteristic of a fungal colony biofilm. Actin is furthermore known to control several cellular processes, such as intracellular movement of 
organelles, vesicular trafficking and cytokinesis ${ }^{46,47}$. Actin has also been proposed as a gravi-perceptor facilitator ${ }^{48}$ since it is involved in re-orientation of direction of movement or growth. Exactly how fungi adapt to different gravitational conditions is still not well understood ${ }^{49}$. To our knowledge, gravity sensing mechanisms in fungi have so far only been studied in Phycomyces, a mucoralean fungus that uses the sedimentation of vacuolar protein crystals and the buoyancy of lipid globules in the hyphal apices to direct the gravitropism of its sporangiophores ${ }^{50-52}$. Previous studies also reported changes in growth of Saccharomyces cereviseae in a bioreactor, presenting random budding, a process to which actin polymerization is central, which was three times higher in weightlessness under microgravity conditions ${ }^{53}$. Future studies will unravel if and how RacA and actin are important for A. niger to adapt to changes to a low gravitational regime.

We furthermore propose that FwnA-mediated melanin production, i.e. pigmentation of $A$. niger's hyphae and spores, plays a role in microgravity response, as cultivation of the mutant under SMG conditions led to increased biomass, biofilm thickness and spore production when compared to cultivation under Ground conditions. Secondary metabolites, such as pigments, are known to be involved a wide-range of cellular processes, from protection from environmental stress (e.g. radiation, ROS, and possibly microgravity) to pathogenicity ${ }^{54,55}$. In $A$. niger, pigments are an important part of the spore surface coating but are also thought to be present in the hyphae and extracellular matrix ${ }^{23,56}$. Deletion of the polyketide synthase FwnA leads to fawn-colored spores ${ }^{42}$, in contrast to white colored spores when the homolog Alb1 is deleted in A. fumigatus ${ }^{57,58}$. Whether $A$. niger spore melanin is formed through the DHN pathway is still a matter of debate. In this study, SEM images reveal that $\triangle f w n A$ spores have less undulated spore surface coating, but are not completely devoid of them, which is in correspondence to data obtained from the $\Delta a l b 1$ mutant of $A$. fumigatus ${ }^{57,58}$. We found that, under normal gravitational conditions, lack of FwnA-mediated pigmentation leads to $50 \%$ increased spore production compared with the wild-type during the first 3 days of colony growth. Interestingly, a previous study suggested that blocking the biosynthetic pathway of melanin may result in an accumulation of the precursor substrate acetyl-CoA which may cause the increase in sporulation in the fungus Pestalotiopsis microspora ${ }^{59}$. Such a putative excess of acetyl-CoA might also help $A$. niger to faster colonize the environment under microgravity conditions, a hypothesis worth studying further in future experiments.

Taken together, our study suggests that the growth of $A$. niger is not inhibited by simulated microgravity, which is promising for fungal-based biotechnology in space. However, it also suggests that $A$. niger's contamination potential of spaceflight environments might increase, given the simulated microgravityinduced increase in spore production and biofilm thickness. In particular, the formation of thicker wildtype biofilms under SMG indicates increased potential for surface colonization and possibly material biodegradation. This can be of concern for spacecraft materials, air- and water systems. For future longterm space missions, the search for strategies and technologies to not only control and mitigate fungal spaceflight biofilms, but also to apply fungal-based biotechnology in spaceflight will be critical ${ }^{21}$. 


\section{MATERIAL AND METHODS}

\section{Strains, media and culture conditions}

Three Aspergillus niger strains were tested in this study and are summarized in Table 1: a fully pigmented wild-type strain (N402), a strain defective in pigment biosynthesis (MA93.1, $\Delta f w n A$ ), and a strain defective in hyperbranching (MA80.1, $\triangle \mathrm{racA}$ ). Spore suspensions were prepared by harvesting spores after 3 days (for N402 and MA93.1) or 5 days (for MA80.1) of growth on complete media (CM) plates [55 mM glucose, $11 \mathrm{mM} \mathrm{KH}_{2} \mathrm{PO}_{4}, 7 \mathrm{mM} \mathrm{KCl}, 178 \mathrm{nM} \mathrm{H}_{3} \mathrm{BO}_{3}, 2 \mathrm{mM} \mathrm{MgSO}_{4}, 76 \mathrm{nM} \mathrm{ZnSO}_{4}, 70 \mathrm{mM} \mathrm{NaNO}_{3}, 6.2 \mathrm{nM} \mathrm{Na}_{2} \mathrm{MoO}_{4}$, $18 \mathrm{nM} \mathrm{FeSO}_{4}, 7.1 \mathrm{nM} \mathrm{CoCl}_{2}, 6.4 \mathrm{nM} \mathrm{CuSO}_{4}, 25 \mathrm{nM} \mathrm{MnCl}_{2}, 174 \mathrm{nM}$ EDTA, $15 \mathrm{~g} / \mathrm{L}$ agar supplemented with $0.5 \%(\mathrm{w} / \mathrm{v})$ yeast extract and $0.1 \%(\mathrm{w} / \mathrm{v})$ casamino acids], and were suspended in $0.9 \%$ sodium chloride. The spore suspensions were then filtered through sterile Miracloth to remove hyphal fragments.

Plates were inoculated with $10 \mu \mathrm{L}$ of $10^{6}$ spores $/ \mathrm{ml}$ spore suspension at the center of minimal medium (MM) plates [55 mM glucose, $11 \mathrm{mM} \mathrm{KH}_{2} \mathrm{PO}_{4}, 7 \mathrm{mM} \mathrm{KCl}, 178 \mathrm{nM} \mathrm{H}_{3} \mathrm{BO}_{3}, 2 \mathrm{mM} \mathrm{MgSO}_{4}, 76 \mathrm{nM} \mathrm{ZnSO}_{4}, 70$ $\mathrm{mM} \mathrm{NaNO}_{3}, 6.2 \mathrm{nM} \mathrm{Na}_{2} \mathrm{MoO}_{4}, 18 \mathrm{nM} \mathrm{FeSO}_{4}, 7.1 \mathrm{nM} \mathrm{CoCl}_{2}, 6.4 \mathrm{nM} \mathrm{CuSO}_{4}, 25 \mathrm{nM} \mathrm{MnCl}_{2}, 174 \mathrm{nM}$ EDTA, 15 $\mathrm{g} / \mathrm{L}$ agar], and grown at $30{ }^{\circ} \mathrm{C}$. Fresh spore suspensions (less than 2 weeks old) were used for all inoculations.

For SEM analysis, a removable polycarbonate-filter $(\varnothing 47 \mathrm{~mm}, 0.4 \mu \mathrm{m}$ pore size, hydrophilic, Merck Millipore, Darmstadt Germany) was placed on top of the agar, before inoculation, to separate the colony from the underlying medium and thus enabling further analysis. Prior to inoculation, all filters were autoclaved in a glass petri dish $\left(121^{\circ} \mathrm{C}, 20 \mathrm{~min}\right)$ and dried overnight at $22^{\circ} \mathrm{C}$. Using sterile tweezers, the filters were carefully placed on $20 \mathrm{ml}$ minimum media agar plates. Colonies were grown under simulated microgravity using the clinostat or under normal gravitational conditions as a control, at $30{ }^{\circ} \mathrm{C}$. For SEM analysis, filters carrying 5-days old colonies were carefully transferred into a small petri dish $(\varnothing 5 \mathrm{~cm})$ using sterile forceps for further sample preparation. There was no change in colony growth with and without the polycarbonate filter (data not shown). For non-SEM measurements, 3-days colonies were used, as their small diameter ensures culture in the central region where there is better quality of microgravity simulation in the Clinostat.

\section{Clinostat cultivation}

Clinostat cultivation to simulate microgravity has been earlier described for Arabidopsis seedlings, Deinococcus radiodurans, S. cerevisiae $e^{60-62}$ and for adherent mammalian cells ${ }^{1}$. The clinostat simulates microgravity through continuous rotation around the horizontal axis (perpendicular to gravity), which averages the gravity vector close to zero, over time, for samples that are located directly in the center of the petri dish ${ }^{35,63}$. This continuous rotation prevents particle sedimentation and exposes cells to a continuous free fall. It is important to note that the clinostat provides a functional simulation which is similar but not identical to that experienced in spaceflight. The quality of simulation is limited to the center of rotation - in this case the very center of the colonies, since as the colony radius increases, acceleration and centrifugal forces increase. A. niger minimal agar plates were rotated at 60 r.p.m. ${ }^{1,64,65}$. For SEM analysis, colonies were divided into two different regions: center and edge (oldest and youngest, 
respectively). To study simulated microgravity-induced effects, only the center region was considered $(r=$ $0.3-0.4 \mathrm{~cm})$, as it is the one exposed to microgravity-like g-forces ranging between $1.2 \times 10^{-2}-1.6 \times 10^{-2} \mathrm{~g}$ $\left(\mathrm{m} \mathrm{s}^{-2}\right)^{66}$ (Figure 1).

\section{Scanning Electron Microscopy}

Colonies grown on minimal medium agar plates covered with polycarbonate filters were chemically fixed, dehydrated and selected regions were freeze-fractured using forceps as previously described ${ }^{67}$. This method preserves the ultrastructure of the fungal colonies, and generates straight fractures in large vertical areas of the colony (cross-section), including major parts of the mycelium and extracellular matrix as well as conidiophore heads. Colonies were fixed on their filter support with $2.5 \%$ glutaraldehyde and $1 \%$ para-formaldehyde in $0.05 \mathrm{M} \mathrm{HEPES}$ buffer, for $12 \mathrm{~h}$ at room temperature. Post-fixation was done with $1 \%$ osmium tetroxide in water before samples were dehydrated in ethanol. During dehydration, colonies usually separated from the filter (without leaving any visible residual colony material on the filter) and some spores were lost. However, the overall integrity of the colony was preserved.

To prepare freezing and fracturing, three representative parts $(\sim 5 \times 3 \mathrm{~mm})$ from center, intermediate and edge region were extracted from each colony (in $100 \%$ ethanol) by using a razor blade. Samples were frozen in liquid nitrogen and fractured according to Fuchs et al. ${ }^{67}$. However, fracturing of samples from the hyperbranching mutant was not successful, most probably because of the thicker vegetative mycelium in comparison to the other strains. We thus fractured samples from the hyperbranching mutant in liquid nitrogen by using a razor blade.

After fracturing, samples were thawed in $100 \%$ ethanol and dried by critical point drying using an automated device (CPD 300, Leica, Wetzlar, Germany). Dried samples were mounted with cross-sections aligned upwards $\left(90^{\circ}\right.$ angle) and sputter-coated with $15 \mathrm{~nm}$ gold/palladium (Sputtercoater E5100, Polaron/Quorum Technologies, UK). Electron microscopy of colony fractures was carried out with a fieldemission scanning electron microscope (Gemini 1530, Zeiss, Oberkochen, Germany) operated at $3 \mathrm{kV}$ and a working distance of $5 \mathrm{~mm}$. Signals from the in-lens secondary electron detector were used to investigate the samples with topography contrast.

SEM image evaluation was done with the iTEM software (Version 5.2; EMSIS, Germany), and with Digimizer image analysis software (MedCalc Software Ltd). Thickness of the vegetative mycelium and height of the conidiophores were determined for the central regions of the colony (at least three measurements per image) in each strain, and for each biological triplicate. Thickness of each vegetative mycelium layer of the $\triangle$ racA strain was determined by four measurements of the central regions per image. Hyperbranching measurements in "fold-regions" were not included in the mycelium thickness analysis, to allow for comparison between strains. 


\section{Transmission electron microscopy}

Small parts (a few millimeters wide and long) of the colonies cultivated under normal gravitational conditions were extracted with a scalpel and sliced in thin sections by using a razor blade. The thin slabs were transferred to the shallow depth of an aluminum platelet ( $3 \mathrm{~mm}$ diameter) filled with hexadecen, covered with another aluminum platelet and fixed by high-pressure freezing. Frozen samples were freezesubstituted in $0.2 \%$ osmium tetroxide, $0.01 \%$ uranyl acetate, $5 \% \mathrm{H}_{2} \mathrm{O}$ in acetone using an automated freeze-substitution device (AFS, Leica Microsystems, Germany) for 3 days and embedded in epon resin. Thin sections (60-80 nm) were produced with an ultramicrotome (UC7, Leica Microsystems, Germany) and contrasted with uranyl acetate and lead citrate. Electron microscopy was performed with a TEM (Tecnai Spirit, Thermo Fisher) operated at 120 kV. Images were recorded with a CCD camera (Megaview III, EMSIS, Germany).

\section{Evaluation of growth assays}

Colony area was determined by taking pictures of the colony and calculating the area through Fiji/Image J by adjusting color and threshold. To determine the amount of spores produced, spores were harvested from single colonies after 3 days in MM-agar plates without filter by flooding the petri dish with $5 \mathrm{~mL}$ of $0.9 \%$ sodium chloride, gently scraping the spores with a sterile cotton swab, and filtering the solution through sterile miracloth to filter out hyphal fragments. Dry biomass was measured by recovering filtergrown colonies, placing them inside pre-weighted aluminum paper, and incubating them for $24 \mathrm{~h}$ at $60^{\circ} \mathrm{C}$ (after which weight was constant), and the dry weight was measured. Dry biomass was calculated as "weight of dried biomass - weight of wet biomass".

\section{Evaluation of spore vitality: hydrophobicity, metabolic activity and germination rate}

To compare the relative hydrophobicity profiles of spores from different $A$. niger strains, the Microbial Adhesion To Hydrocarbons (MATH) assay was adapted. Spores isolated from minimal agar plates were suspended in $0.9 \%$ sodium chloride and exposed to hexadecane, an apolar solvent, that allows spores to settle in either the aqueous or the organic phase, depended on which more strongly interacts with the spore surface ${ }^{68,69}$. This semi-quantitative approach provides evidence of the surface hydrophobicity of the spores via their interactive properties. For that, $2.5 \mathrm{ml}$ of spore suspension $\left(1 \times 10^{6} \mathrm{spores} / \mathrm{ml}\right)$ were added to glass tubes and $5 \mathrm{ml}$ hexadecane was slowly adding on top. The tubes were vortexed for $2 \mathrm{~min}$, followed by a 15 min settling phase, after which $1 \mathrm{ml}$ sample of the suspension was taken carefully from the bottom of the tube (through the hexadecane layer). Measurements of spore suspensions at $\mathrm{OD}_{600 \mathrm{~nm}}$ were taken before $\left(N_{0}\right)$ and after contact with hexadecane $(N)$. Hydrophobicity was calculated as $\left[\left(\left(N / N_{0}\right) \times 100\right)-100\right]$, which determines the percentage of spores in the hydrocarbon layer - i.e.; the higher the percentage of spores in the hydrocarbon layer, the higher the hydrophobicity. This experiment was performed two independent times, each with three technical replicates. 
Spore germination rate was determined by inoculating $3 \mu \mathrm{L}$ of $10^{6}$ spores $/ \mathrm{ml}$ in MM supplemented with $0.003 \%$ yeast extract. After incubation at $22{ }^{\circ} \mathrm{C}$ for $22 \mathrm{~h}$, light microscopy allowed for counting of 200 spores and germlings per replicate, per strain. Germination rate was calculated as the ration (N/NG) between germinated (G) and non-germinated spores (NG).

Metabolic activity of spores was measured with the Alamar Blue assay in a 96-well plate. In each well a total volume of $300 \mu \mathrm{L}$ were pipetted [195 $\mu \mathrm{l}$ of $\mathrm{MM}, 75 \mu \mathrm{L}$ spore suspension $\left(10^{6}\right.$ spores $\left./ \mathrm{ml}\right), 30 \mu \mathrm{L}$ of Alamar Blue (Sigma)]. Spores were incubated for max $48 \mathrm{~h}$ at $30^{\circ} \mathrm{C}$ in a TECAN plate reader $(n=6)$. The color changes when spore start to germinate and thus become able to metabolize resazurin (blue) into resorufin (pink). This change was detected by $\mathrm{OD}_{570 \mathrm{~nm}}$ and $\mathrm{OD}_{600 \mathrm{~nm}}$.

\section{Data analysis}

Data were plotted as Mean and error bars calculated as Standard Error. Graphs were plotted with Sigma Plot.14. Statistical analysis was done with Student's t-test, using Mean and Standard-Error between Ground and SMG conditions, or between a mutant strain and the wild-type. A one-tailed $p$-value of $p \leq$ 0.05 was considered as significant.

\section{ACKNOWLEDGEMENTS}

We are very grateful to Ruth Hemmersbach and DLR's Gravitation Biology Department for providing the clinostats, excellent technical support, and advice on clinostat operations. R.M. and M.C. received funding from the following DLR grants: ISS LIFE (Programm RF-FuW, Teilprogramm: 475), AEROMASK, NGT-BIT (DLR VandE: Next Generation Train), simplAIR (Programm LF (simpIAIR ME / KoPa33), Teilbereich: 251). M.C. doctoral thesis was supported by DLR-DAAD Research fellowship (57370122). The research project at the Robert Koch Institute (RKI) in Berlin was supported by a DGE (Deutsche Gesellschaft für Elektronenmikroskopie) scholarship, which was awarded to M.C.

\section{AUTHOR CONTRIBUTIONS}

M.C. designed and performed the experiments and analyzed the data. G.H. and M.L. assisted with SEM experimental design, sample preparation, imaging and analysis. M.C., T.S., V.M. and R.M. designed the study and jointly interpreted the data. M.C. and V.M. co-wrote the manuscript which was approved by all co-authors. 


\section{COMPETING INTERESTS}

The authors declare no competing interests.

\section{REFERENCES}

1. Eiermann, P. et al. Adaptation of a 2-D Clinostat for simulated microgravity experiments with adherent cells. Microgravity Sci. Technol. 25, 153-159 (2013).

2. Bizzarri, M., Monici, M. and Loon, J. J. W. A. V. How microgravity affects the biology of living systems. Biomed Res. Int. 2015, (2015).

3. Horneck, G. et al. Space Microbiology. Annu. Rev. Microbiol. 74, 121-137 (2010).

4. Rosenzweig, J. a. et al. Spaceflight and modeled microgravity effects on microbial growth and virulence. Appl. Microbiol. Biotechnol. 85, 885-891 (2010).

5. Taylor, P. W. Impact of space flight on bacterial virulence and antibiotic susceptibility. Infect. Drug Resist. 249-262 (2015) doi:10.2147/IDR.S67275.

6. Gilbert, R. et al. Spaceflight and simulated microgravity conditions increase virulence of Serratia marcescens in the Drosophila melanogaster infection model. npj Microgravity 6, 4 (2020).

7. Hammond, T. G. et al. Effects of microgravity on the virulence of Listeria monocytogenes, Enterococcus faecalis, Candida albicans, and methicillin-resistant Staphylococcus aureus. Astrobiology 13, 1081-90 (2013).

8. Novikova, N. et al. Survey of environmental biocontamination on board the International Space Station. Res. Microbiol. 157, 5-12 (2006).

9. Cairns, T. C., Nai, C. and Meyer, V. How a fungus shapes biotechnology: 100 years of Aspergillus niger research. Fungal Biol. Biotechnol. 5, 13 (2018).

10. Meyer, V. et al. Growing a circular economy with fungal biotechnology: a white paper. Fungal biology and biotechnology vol. 75 (2020).

11. Sugui, J. A., Kwon-Chung, K. J., Juvvadi, P. R., Latgé, J.-P. and Steinbach, W. J. Aspergillus fumigatus and related species. Cold Spring Harb. Perspect. Med. 5, a019786 (2014).

12. Novikova, N. D. Review of the knowledge of microbial contamination of the Russian manned spacecraft. Microb. Ecol. 47, 127-132 (2004).

13. Klintworth, R., Reher, H. J., Viktorov, A. N. and Bohle, D. Biological induced corrosion of materials II: new test methods and experiences from MIR station. Acta Astronaut. 44, 569-578 (1999).

14. Yamaguchi, N. et al. Microbial monitoring of crewed habitats in space-current status and future 
perspectives. Microbes Environ. 29, 250-260 (2014).

15. Di Pippo, F., Di Gregorio, L., Congestri, R., Tandoi, V. and Rossetti, S. Biofilm growth and control in cooling water industrial systems. FEMS Microbiol. Ecol. 94, (2018).

16. Airliner, T. et al. The Airliner Cabin Environment and the Health of Passengers and Crew. The Airliner Cabin Environment and the Health of Passengers and Crew (2002). doi:10.17226/10238.

17. Makimura, K. et al. Fungal flora on board the Mir-Space Station, identification by morphological features and ribosomal DNA sequences. Microbiol. Immunol. 45, 357-63 (2001).

18. Vesper, S. J., Wong, W., Kuo, C. M. and Pierson, D. L. Mold species in dust from the International Space Station identified and quantified by mold-specific quantitative PCR. Res. Microbiol. 159, 432-435 (2008).

19. Cortesão, M. et al. Aspergillus niger Spores Are Highly Resistant to Space Radiation. Front. Microbiol. 11, 1-12 (2020).

20. Zea, L. et al. Potential biofilm control strategies for extended spaceflight missions. Biofilm 2, 100026 (2020).

21. Cortesão M., Schütze T., Marx R., Moeller R., Meyer V. Fungal biotechnology in Space: Why and how?. In: Nevalainen H. (eds) Grand Challenges in Fungal Biotechnology. Grand Challenges in Biology and Biotechnology. Springer, Cham. (2020) https://doi.org/10.1007/978-3-030-295417_18

22. Chung, K. Y. and Brown, J. C. S. Biology and function of exo-polysaccharides from human fungal pathogens. Curr. Clin. Microbiol. reports 7, 1-11 (2020).

23. Beauvais, A. et al. An extracellular matrix glues together the aerial-grown hyphae of Aspergillus fumigatus. Cell. Microbiol. 9, 1588-1600 (2007).

24. Lagree, K. and Mitchell, A. P. Fungal biofilms: Inside out. The Fungal Kingdom 873-886 (2017) doi:10.1128/microbiolspec.funk-0024-2016.

25. Beauvais, A. and Latgé, J.-P. Aspergillus biofilm in vitro and in vivo. Microbiol. Spectr. 3, MB-0017 (2015).

26. Mela, A. P., Rico-Ramírez, A. M. and Glass, N. L. Syncytia in Fungi. Cells vol. 9 (2020).

27. Beauvais, A., Fontaine, T., Aimanianda, V. and Latgé, J. P. Aspergillus cell wall and biofilm. Mycopathologia 178, 371-377 (2014).

28. Sheppard, D. C. and Howell, P. L. Biofilm exopolysaccharides of pathogenic fungi: Lessons from bacteria*. J. Biol. Chem. 291, 12529-12537 (2016).

29. Krijgsheld, P. et al. Development in Aspergillus. Stud. Mycol. 74, 1-29 (2013). 
30. Romsdahl, J. et al. Characterization of Aspergillus niger isolated from the International Space Station . mSystems 3, 1-13 (2018).

31. Blachowicz, A. et al. Proteomic and metabolomic characteristics of extremophilic fungi under simulated Mars conditions. Front. Microbiol. 10, 1-16 (2019).

32. Sathishkumar, Y. et al. Effect of low shear modeled microgravity on phenotypic and central chitin metabolism in the filamentous fungi Aspergillus niger and Penicillium chrysogenum. Antonie van Leeuwenhoek, Int. J. Gen. Mol. Microbiol. 106, 197-209 (2014).

33. Sathishkumar, Y., Krishnaraj, C., Rajagopal, K., Sen, D. and Lee, Y. S. High throughput de novo RNA sequencing elucidates novel responses in Penicillium chrysogenum under microgravity. Bioprocess Biosyst. Eng. 39, 223-231 (2016).

34. Klaus, D. M. Clinostats and bioreactors. Gravit. Space Biol. Bull. 14, 55-64 (2001).

35. Hasenstein, K. H. and van Loon, J. J. W. A. Clinostats and other rotating systems-design, function, and limitations. Gener. Appl. Extra-Terrestrial Environ. Earth 147-156 (2015).

36. Jiang, C. et al. Clinostat rotation affects metabolite transportation and increases organic acid production by Aspergillus carbonarius, as revealed by differential metabolomic analysis. Appl. Environ. Microbiol. 85, 1-45 (2019).

37. Fiedler, M. R. M., Barthel, L., Kubisch, C., Nai, C. and Meyer, V. Construction of an improved Aspergillus niger platform for enhanced glucoamylase secretion. Microb. Cell Fact. 17, 95 (2018).

38. Kikuma, T., Arioka, M. and Kitamoto, K. Autophagy during conidiation and conidial germination in filamentous fungi. Autophagy 3, 128-129 (2007).

39. Nitsche, B. M., Burggraaf-van Welzen, A.-M., Lamers, G., Meyer, V. and Ram, A. F. J. Autophagy promotes survival in aging submerged cultures of the filamentous fungus Aspergillus niger. Appl. Microbiol. Biotechnol. 97, 8205-8218 (2013).

40. Tiedt, L. R. An electron microscope study of conidiogenesis and wall formation of conidia of Aspergillus niger. Mycol. Res. 97, 1459-1462 (1993).

41. Bos, C. J. et al. Genetic analysis and the construction of master strains for assignment of genes to six linkage groups in Aspergillus niger. Curr. Genet. 14, 437-443 (1988).

42. Jørgensen, T. R. et al. The molecular and genetic basis of conidial pigmentation in Aspergillus niger. Fungal Genet. Biol. 48, 544-553 (2011).

43. Kwon, M. J. et al. Functional characterization of Rho GTPases in Aspergillus niger uncovers conserved and diverged roles of Rho proteins within filamentous fungi. Mol. Microbiol. 79, 11511167 (2011).

44. Boswell, G. P. and Hopkins, S. Linking hyphal growth to colony dynamics: Spatially explicit models of mycelia. Fungal Ecol. 1, 143-154 (2008). 
45. Adams, T. H., Wieser, J. K. and Yu, J. H. Asexual sporulation in Aspergillus nidulans. Microbiol. Mol. Biol. Rev. 62, 35-54 (1998).

46. Walker, S. K. and Garrill, A. Actin microfilaments in fungi. Mycologist 20, 26-31 (2006).

47. Bergs, A., Ishitsuka, Y., Evangelinos, M., Nienhaus, G. U. and Takeshita, N. Dynamics of actin cables in polarized growth of the filamentous fungus Aspergillus nidulans. Front. Microbiol. 7, 115 (2016).

48. Velle, K. B. and Fritz-Laylin, L. K. Diversity and evolution of actin-dependent phenotypes. Curr. Opin. Genet. Dev. 58-59, 40-48 (2019).

49. Häder, D.-P. Gravitropism in Fungi, Mosses and Ferns BT - Gravitational Biology I: Gravity Sensing and Graviorientation in Microorganisms and Plants. in (eds. Braun, M., Böhmer, M., Häder, D.-P., Hemmersbach, R. and Palme, K.) 67-74. Springer International Publishing (2018). doi:10.1007/978-3-319-93894-3_5.

50. Grolig, F., Döring, M. and Galland, P. Gravisusception by buoyancy: A mechanism ubiquitous among fungi? Protoplasma 229, 117-123 (2006).

51. Moore, D. Graviresponses in fungi. Adv. Sp. Res. 17, 73-82 (1996).

52. Schimek, C. et al. Gravitropism in Phycomyces: a role for sedimenting protein crystals and floating lipid globules. Planta 210, 132-142 (1999).

53. Walther, I., Bechler, B., Müller, O., Hunzinger, E. and Cogoli, A. Cultivation of Saccharomyces cerevisiae in a bioreactor in microgravity. J. Biotechnol. 47, 113-127 (1996).

54. Cordero, R. J. and Casadevall, A. Functions of fungal melanin beyond virulence. Fungal Biol. Rev. 31, 99-112 (2017).

55. Lin, L. and Xu, J. Fungal pigments and their roles associated with human health. J. fungi (Basel, Switzerland) 6, 280 (2020).

56. Bayry, J. et al. Surface structure characterization of Aspergillus fumigatus conidia mutated in the melanin synthesis pathway and their human cellular immune response. Infect. Immun. 82, 31413153 (2014).

57. Pihet, M. et al. Melanin is an essential component for the integrity of the cell wall of Aspergillus fumigatus conidia. BMC Microbiol. 9, 1-11 (2009).

58. Paris, S. et al. Conidial hydrophobins of Aspergillus fumigatus. Appl. Environ. Microbiol. 69, 15811588 (2003).

59. Yu, X. et al. Melanin is required for the formation of the multi-cellular conidia in the endophytic fungus Pestalotiopsis microspora. Microbiol. Res. 179, 1-11 (2015).

60. Fengler, S. et al. Changes in gene expression of Arabidopsis thaliana cell cultures upon exposure 
to real and simulated partial-g forces. Microgravity Sci. Technol. 28, 319-329 (2016).

61. Ott, E. et al. Molecular response of Deinococcus radiodurans to simulated microgravity explored by proteometabolomic approach. Sci. Rep. 9, 1-13 (2019).

62. Van Mulders, S. E. et al. The influence of microgravity on invasive growth in Saccharomyces cerevisiae. Astrobiology 11, 45-55 (2011).

63. Herranz, R. et al. Ground-based facilities for simulation of microgravity: Organism-specific recommendations for their use, and recommended terminology. Astrobiology 13, 1-17 (2013).

64. Garschagen, L. S., Mancinelli, R. L. and Moeller, R. Introducing Vibrio natriegens as a microbial model organism for microgravity research. Astrobiology 19, 1211-1220 (2019).

65. Krause, L., Braun, M., Hauslage, J. and Hemmersbach, R. Analysis of statoliths displacement in chara rhizoids for validating the microgravity-simulation quality of clinorotation modes.

Microgravity Sci. Technol. 30, 229-236 (2018).

66. Ritzmann, R., Freyler, K., Krause, A. and Gollhofer, A. Bouncing on Mars and the Moon-the role of gravity on neuromuscular control: Correlation of muscle activity and rate of force development. J. Appl. Physiol. 121, 1187-1195 (2016).

67. Fuchs, F. M., Holland, G., Moeller, R. and Laue, M. Directed freeze-fracturing of Bacillus subtilis biofilms for conventional scanning electron microscopy. J. Microbiol. Methods 152, 165-172 (2018).

68. Shuster, B. et al. Contributions of crust proteins to spore surface properties in Bacillus subtilis. Mol. Microbiol. 111, 825-843 (2019).

69. Faille, C. et al. Presence and function of a thick mucous layer rich in polysaccharides around Bacillus subtilis spores. Biofouling 30, 845-858 (2014). 


\section{FIGURE LEGENDS}

Figure 1. Simulation of microgravity in a petri-dish clinostat and effect of simulated microgravity in colony area. a Colonies were grown on a filter that covered minimal medium agar in Petri-dishes. The plates fit into the clinostat that simulates microgravity by continuously rotating the petri dishes (60 r.p.m.). Here, the central region of the colony can achieve g-forces from $1.2 \times 10-2$ to $1.6 \times 10-2 \mathrm{~g}$, in a colony $(r)$ radius between $0.3-0.4 \mathrm{~cm}$. Colonies were sampled for SEM in the center and at the edge. $b$ Pictures of representative colonies of the three tested strains after 5 days of cultivation $(\mathrm{scale}=1 \mathrm{~cm})$.

Figure 2. Simulated microgravity induces changes in A. niger colony area, dry biomass area, spore production and spore hydrophobicity in a strain-dependent manner. Spores of the three test strains were used to inoculate minimal medium agar plates as biological triplicates. After three days of incubation, several morphological characteristics were determined. a Colony area. b Dry biomass. c Spore production per colony. $d$ Spore hydrophobicity. Data shown as mean with error bars as standard error, where $p \leq 0.05$ was considered significant and is indicated with an asterisk.

Figure 3. SEM images of Aspergillus niger wild-type colonies grown in static, aerial conditions. a vegetative $(\mathrm{Vm})$ and aerial mycelia $(\mathrm{Am})$ with conidiophores. b close-up view of the interface of aerial and vegetative mycelium showing extracellular matrix (ECM), stalks (st), loose spores (sp) and vegetative hyphae (h). c ECM that covered vegetative mycelium in the center region of the colony. $d$ Hollow hyphae in the center region. $e, f$ hyphae at the edge of the colony not yet covered with ECM. $f$ close-up view of compact hyphae filled with cytoplasm at the colony edge. g cross-section of $A$. niger conidiophore revealing stalk, vesicle, metulae, phialides and conidia chains. $h$, i close-up view of phialides and spore chains, revealing folded outer layer of spores.

Figure 4. SEM images of A. niger strains from the central region of colonies grown in Ground and simulated microgravity (SMG) conditions. Representative images of colony fragment cross-sections showing differences in vegetative and aerial mycelia for the tested strains. Note that the thickness of the vegetative mycelium and the height of conidiophores differs among the strains. Scale is the same for all images.

Figure 5. Vegetative mycelium ultrastructure of different $A$. niger strains grown under Ground and simulated microgravity (SMG) conditions. Note that the thickness of the vegetative mycelium differs between the strains. Two or three layers of the vegetative mycelium can be distinguished in a straindependent manner and are indicated with numbers 1, 2 and 3. Scale is the same for all images in each column.

Figure 6. Conidiophores and spore chains of $A$. niger strains under Ground and simulated microgravity (SMG) conditions. Note that the length of the spore chains differs between strains, which can be up to $150 \mu \mathrm{m}$ in the $\triangle f w n A$ mutant. Scale is the same for all images in each column.

Figure 7. Spore morphology and vitality from A. niger colonies grown under Ground and simulated microgravity (SMG) conditions. a SEM of spores of each tested strain show no morphological differences when produced under SMG. Differences between spore surfaces of wild-type and $\triangle$ fwnA mutant can be seen, as a defect in pigmentation leads to changes in the outer layer of the cell wall b Metabolism upon germination show no significant changes under SMG. Data shown as mean from biological triplicates with error bars. 


\section{TABLES}

Table 1. Strains used in this study.

\begin{tabular}{llll}
\hline Strain name & $\begin{array}{l}\text { Relevant } \\
\text { genotype }\end{array}$ & Characteristics & Reference \\
\hline N402 & Wild-type & Fully pigmented spores & (Bos et al. 1988) \\
MA93.1 & $\Delta f w n A$ & $\begin{array}{l}\text { Pigmentation mutant that produces } \\
\text { fawn-colored spores due to the lack of } \\
\text { the polyketide synthase FwnA }\end{array}$ & (Jørgensen et al. 2011) \\
& & Hyperbranching mutant due to the & (Kwon et al. 2011) \\
MA80.1 & lack of the Rho GTPase RacA & \\
\hline
\end{tabular}




\section{Supplemental Material}

\section{Colony growth and biofilm formation of Aspergillus niger under simulated}

\section{microgravity}

Marta Cortesao ${ }^{1,2 *}$, Gudrun Holland ${ }^{3}$, Tabea Schütze ${ }^{2}$, Michael Laue ${ }^{3}$, Ralf Moeller ${ }^{1}$ and Vera Meyer $^{2}$

${ }^{1}$ German Aerospace Center (DLR), Institute of Aerospace Medicine, Radiation Biology Department, Aerospace Microbiology Research Group, Cologne, Germany

${ }^{2}$ Chair of Applied and Molecular Microbiology, Institute of Biotechnology, Technische Universität Berlin, Berlin, Germany

${ }^{3}$ Robert Koch Institute, Advanced Light and Electron Microscopy (ZBS 4), Berlin, Germany

* Corresponding author: E-mail: marta.cortesao@dlr.de, Phone +49(2203) 601-4211 
a

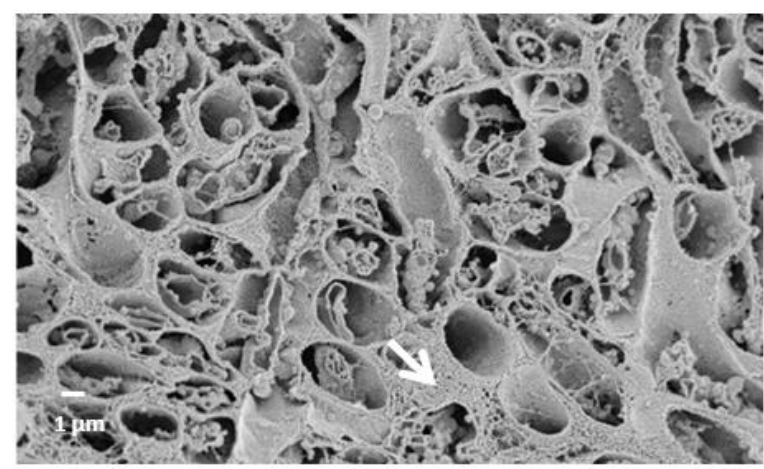

b

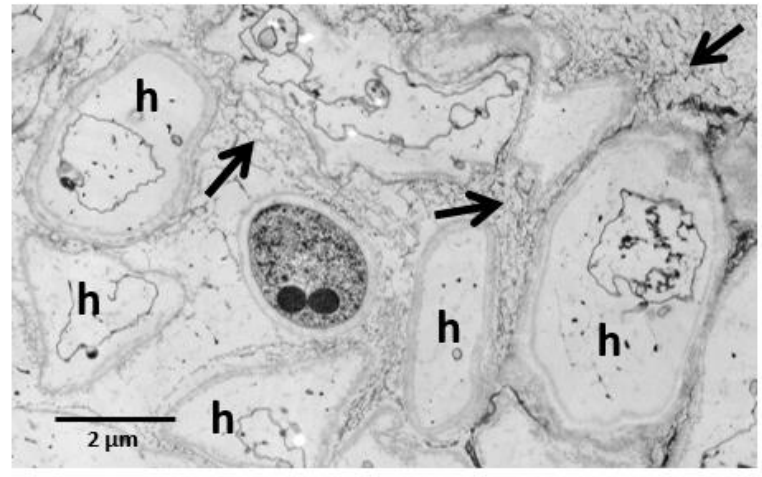

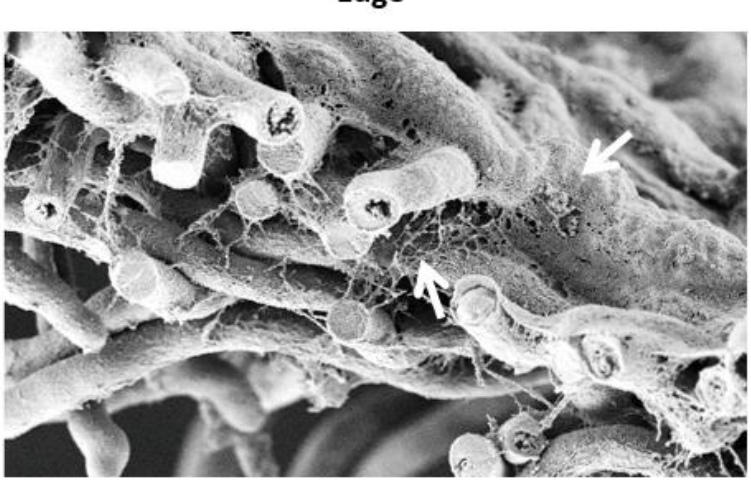

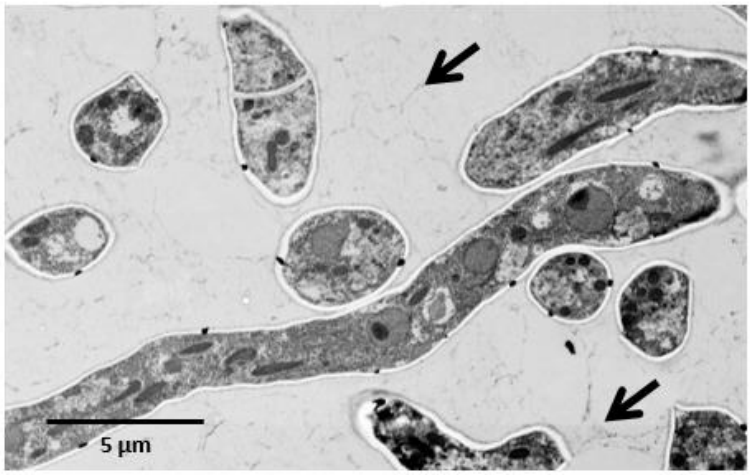

Supplemental Figure 1. Comparison of the ultrastructural preservation of A. niger hyphae in different colony regions after chemical or cryo-fixation. a SEM of chemically fixed samples. Cross-sections through the colony show empty hyphae in the center of a colony, and filled hyphae at the edge region of a colony. $b$ TEM of ultrathin sections of high-pressure frozen samples show almost empty hyphae (exception labeled with an asterisk) in the center of the colony while at the edge hyphae reveal a dense, intact cytoplasm with all typical organelles. Arrows indicate the extracellular matrix formed. 


\section{Wild-type}

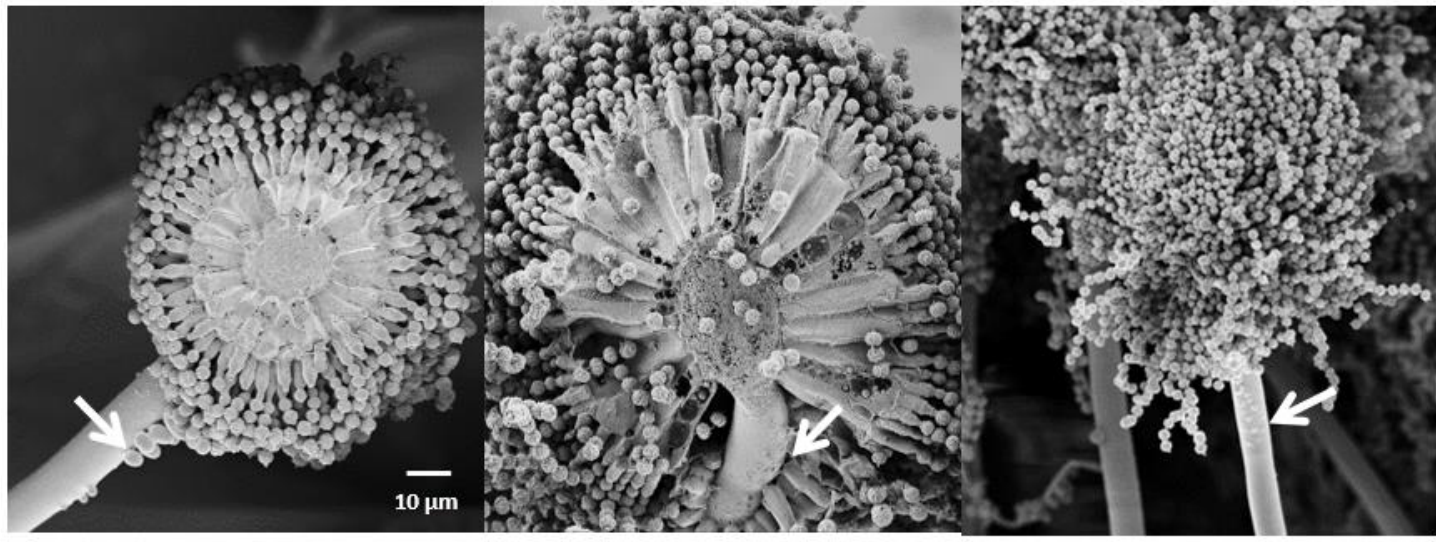

Pigmentation mutant

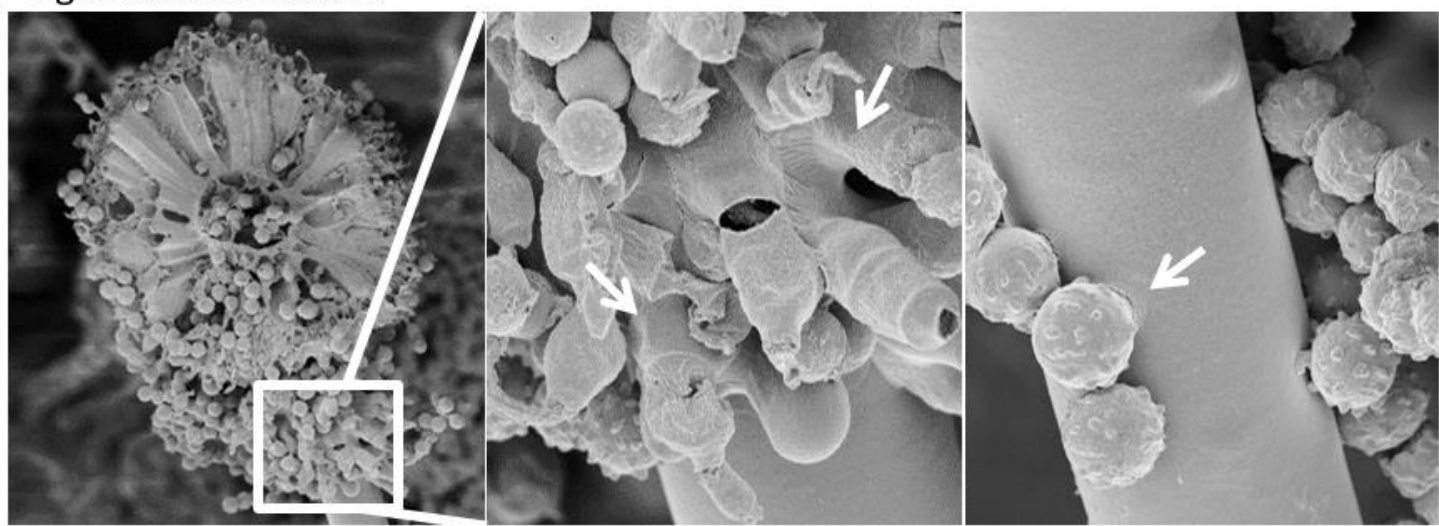

Hyperbranching mutant

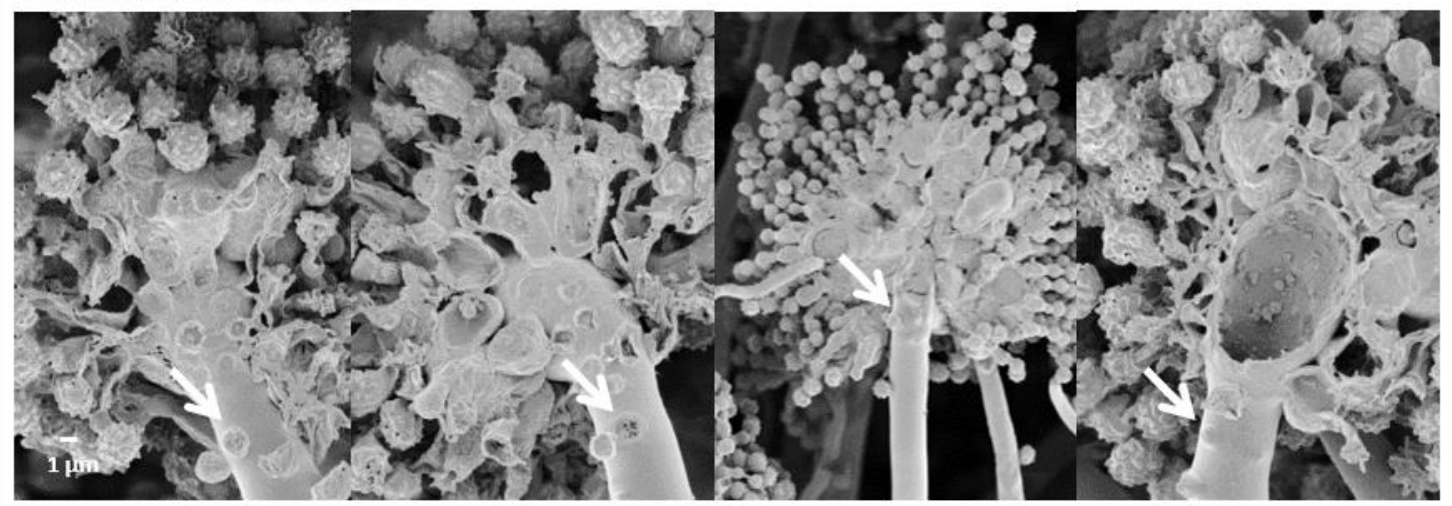

Supplemental Figure 2. Conidiophore morphologies of A. niger strains. Arrows indicate irregular spore formation, through the conidiophore stalk (rather than through the vesicle) which occurred seldomly in the wild-type and $\Delta f w n A$ mutant but frequently in the $\triangle$ racA mutant. 

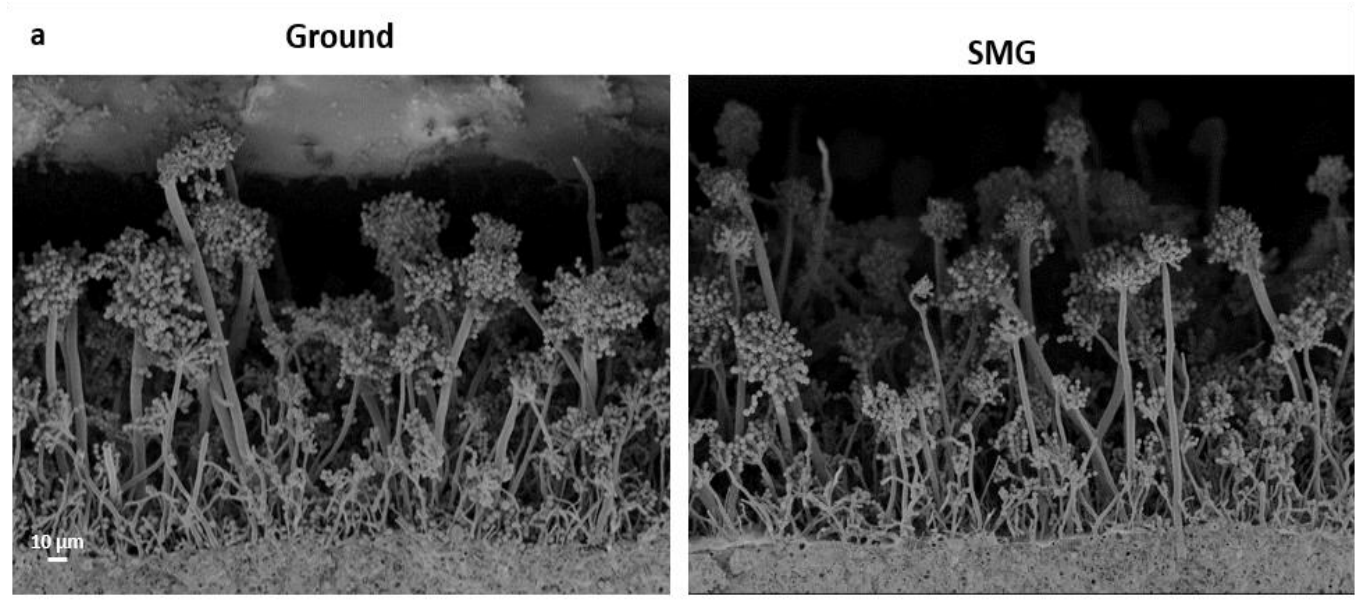

b
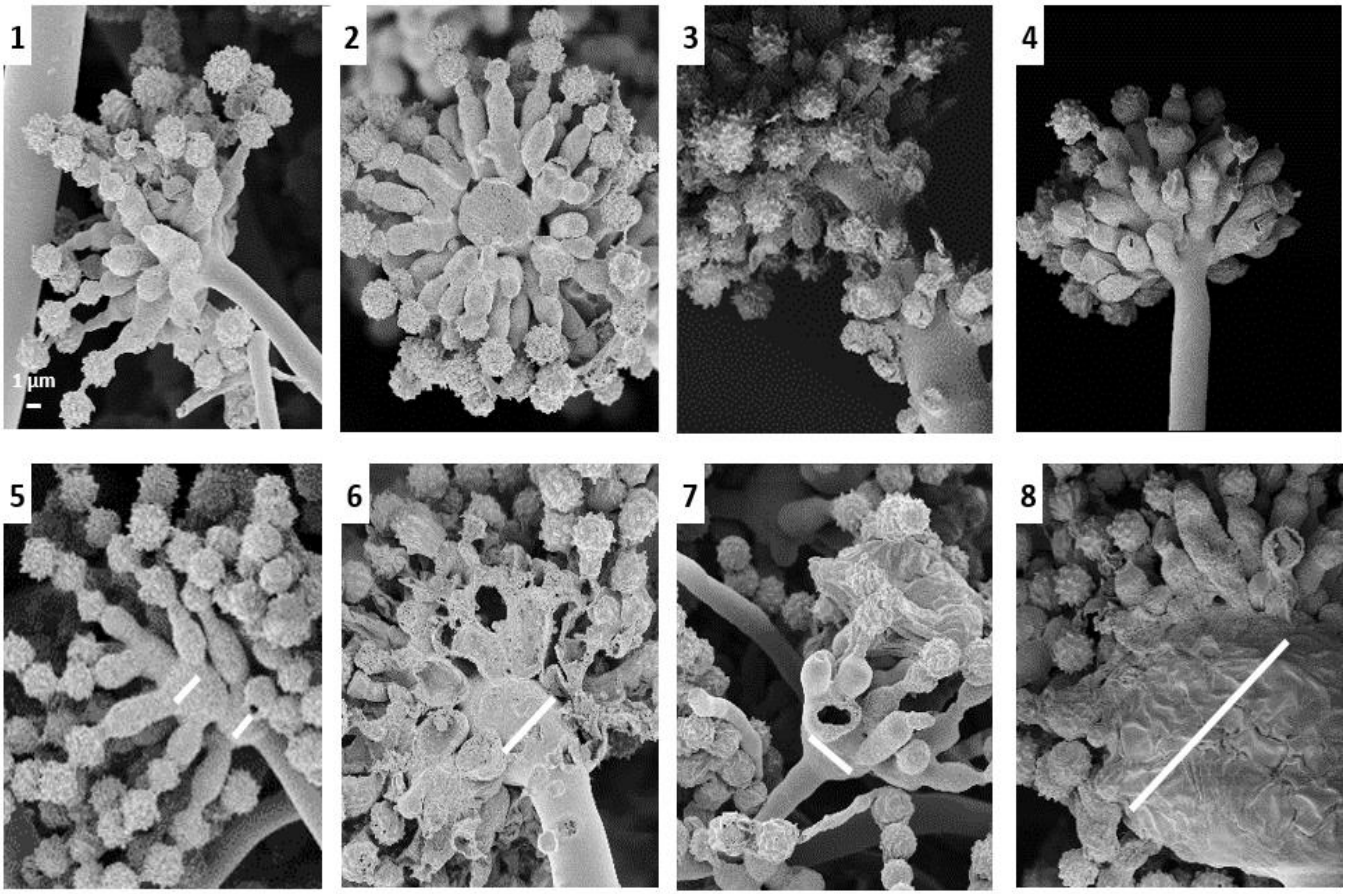

Supplemental Figure 3. Irregular conidiophore morphologies in the hyperbranching mutant strain $\Delta r a c A$. a Aerial mycelium showing conidiophores at different heights and morphologies. b Irregular vesicle formation: no vesicle (1-4), doubled vesicle (5) and vesicle with a divergent width (white line, 6-8). Irregularities were observed regardless of the gravitational regime. 
Ground
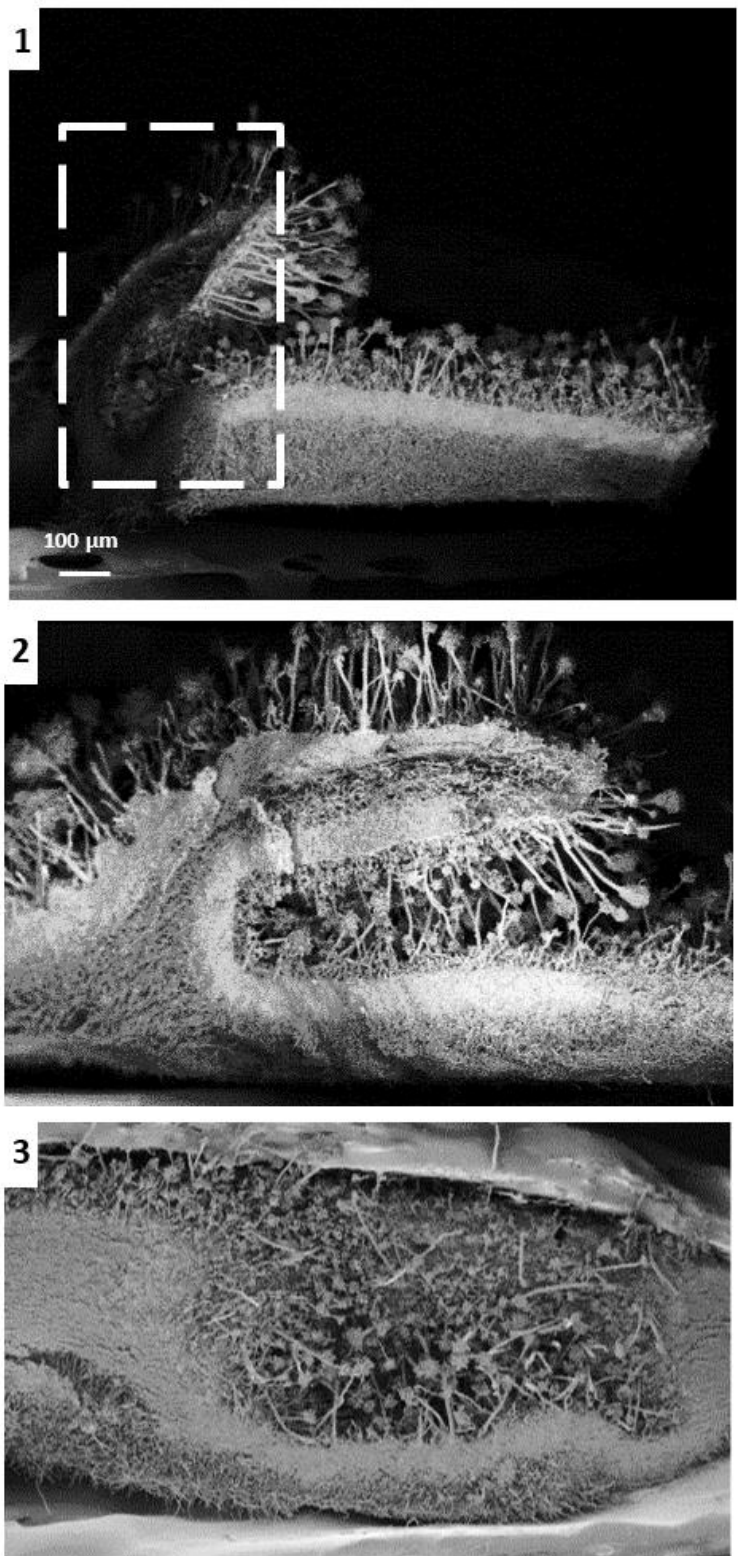

SMG
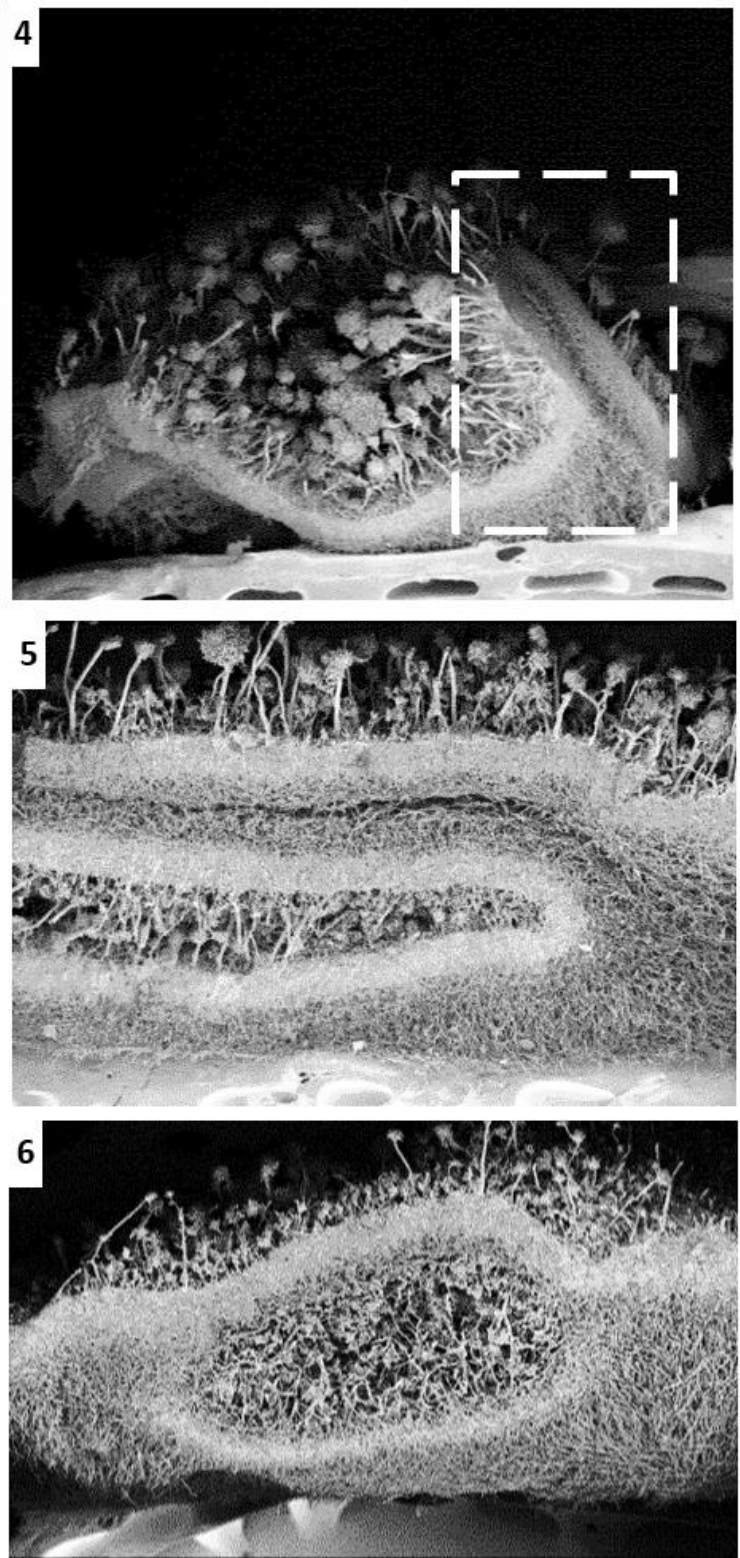

Supplemental Figure 4. Irregular colony morphologies in the hyperbranching mutant strain $\Delta$ racA. Representative pictures of colony cross-sections show mycelium folds in both Ground (1-3) and simulated gravity conditions (4-6) after 5 days of cultivation on minimal medium agar plates. Note that aerial hyphae and conidiophores are formed in both directions: upward and downward. 
Supplemental Table 1. Effect of simulated microgravity in A. niger colony biofilms. Three strains were tested: wildtype, pigmentation $(\triangle f w n A)$ and hyperbranching $(\Delta r a c A)$ mutant strains.

\begin{tabular}{|c|c|c|c|c|c|}
\hline Parameter & Strain & Ground & SMG & $\neq$ & p-value \\
\hline \multicolumn{6}{|c|}{ Colony Area $\left(\mathrm{cm}^{2}\right)$} \\
\hline & Wild-type & $3.3 \pm 0.3$ & $3.7 \pm 0.2$ & $\uparrow$ & 0.3 \\
\hline & Pigmentation & $3.7 \pm 0.2$ & $4.4 \pm 0.3$ & $\uparrow$ & $0.07 *$ \\
\hline & Hyperbranching & $1.2 \pm 0.0$ & $1.3 \pm 0.0$ & $\uparrow$ & 0.3 \\
\hline \multicolumn{6}{|c|}{ Dried biomass (mg) } \\
\hline & Wild-type & $8.7 \pm 2.6$ & $9,7 \pm 0.9$ & $=$ & 0.7 \\
\hline & Pigmentation & $6.0 \pm 1.0$ & $8.7 \pm 1.5$ & $\uparrow$ & 0.2 \\
\hline & Hyperbranching & $9.7 \pm 1.8$ & $6.3 \pm 0.7$ & $\downarrow$ & 0.1 \\
\hline \multicolumn{6}{|c|}{ Spores per colony } \\
\hline & Wild-type & $6.0 \times 10^{7} \pm 2.4 \times 10^{6}$ & $7.0 \times 10^{7} \pm 6.2 \times 10^{6}$ & $\uparrow$ & 0.2 \\
\hline & Pigmentation & $9.0 \times 10^{7} \pm 4.5 \times 10^{6}$ & $1.1 \times 10^{8} \pm 5.4 \times 10^{6}$ & $\uparrow$ & $0.03 *$ \\
\hline & Hyperbranching & $6.8 \times 10^{6} \pm 1.3 \times 10^{5}$ & $9.1 \times 10^{6} \pm 7.1 \times 10^{5}$ & $\uparrow$ & $0.03 *$ \\
\hline \multicolumn{6}{|c|}{ Spore metabolic max (\%) } \\
\hline & Wild-type & $17 \pm 0.4$ & $15 \pm .09$ & $\downarrow$ & 0.1 \\
\hline & Pigmentation & $16 \pm 1.1$ & $17 \pm 0.3$ & $=$ & 0.7 \\
\hline & Hyperbranching & $19 \pm 0.6$ & $19 \pm 1.1$ & $=$ & 0.8 \\
\hline \multicolumn{6}{|c|}{ Spore metabolic max (h) } \\
\hline & Wild-type & $25 \pm 0.4$ & $24 \pm 0.4$ & $\uparrow$ & 0.15 \\
\hline & Pigmentation & $26 \pm 0.3$ & $26 \pm 0.4$ & $=$ & 1 \\
\hline & Hyperbranching & $24 \pm 0.6$ & $23 \pm 0.4$ & $\downarrow$ & 0.2 \\
\hline \multicolumn{6}{|c|}{ Spore germination (G/NG) } \\
\hline & Wild-type & $10 \pm 0.4$ & $5.6 \pm 1$ & $\downarrow$ & $0.02 *$ \\
\hline & Pigmentation & $15 \pm 1.1$ & $15 \pm 2.4$ & $=$ & 0.9 \\
\hline \multicolumn{6}{|c|}{ Spore hydrophobicity (\%) } \\
\hline & Wild-type & $49 \pm 3$ & $51 \pm 15$ & $=$ & 0.9 \\
\hline & Pigmentation & $31 \pm 4$ & $34 \pm 7$ & $=$ & 0.7 \\
\hline & Hyperbranching & $58 \pm 15$ & $90 \pm 3$ & $\uparrow$ & 0.1 \\
\hline \multicolumn{6}{|c|}{$\begin{array}{l}\text { Biofilm thickness in colony } \\
\text { center }(\mu \mathrm{m})\end{array}$} \\
\hline & Wild-type & $59 \pm 1$ & $72 \pm 1$ & $\uparrow$ & $0.001 *$ \\
\hline & Pigmentation & $67 \pm 2$ & $63 \pm 1$ & $\downarrow$ & 0.1 \\
\hline & Hyperbranching & $176 \pm 10$ & $275 \pm 31$ & $\uparrow$ & $0.04 *$ \\
\hline \multicolumn{6}{|c|}{ Conidiophore height $(\mu \mathrm{m})$} \\
\hline & Wild-type & $466 \pm 35$ & $417 \pm 35$ & $\downarrow$ & 0.4 \\
\hline & Pigmentation & $454 \pm 34$ & $510 \pm 41$ & $\uparrow$ & 0.4 \\
\hline & Hyperbranching & $174 \pm 17$ & $165 \pm 11$ & $=$ & 0.7 \\
\hline
\end{tabular}

*Statistical significance with Students t-test where one-tailed $p \leq 0.05$. 


\section{Chapter IV: Design of a spaceflight biofilm experiment; Controlling spaceflight fungal biofilms}

\section{Publication:}

Zea, L., Nisar, Z., Rubin, P., Cortesao, M., Luo, J., McBride, S., Moeller, R., Klaus, D., Mueller, D., Varanasi, K., Mücklich, F., Stodieck L. "Design of a spaceflight biofilm experiment". Acta Astronautica 148, 294-300, $\underline{2018}$

\section{Author contribution}

Planning: $15 \%$, Experiment: $40 \%$, Manuscript: $15 \%$

\section{Publication:}

Cortesao, M., Rubin, P., Luo, J., Hellweg, C.E., Stodieck L. Mücklich, F., Klaus, D., Moeller, R., and Zea, L. "Controlling spaceflight fungal biofilms: the search for antimicrobial surfaces". International

Astronautical Congress (IAC) 2018

\section{Author contribution}

Planning: $60 \%$, Experiment: $80 \%$, Manuscript: $80 \%$ 


\section{Design of a spaceflight biofilm experiment}

Luis Zea ${ }^{\mathrm{a}, *}$, Zeena Nisar ${ }^{\mathrm{a}}$, Phil Rubin ${ }^{\mathrm{a}}$, Marta Cortesão ${ }^{\mathrm{b}}$, Jiaqi Luo ${ }^{\mathrm{c}}$, Samantha A. McBride ${ }^{\mathrm{d}}$, Ralf Moeller ${ }^{\mathrm{b}}$, David Klaus ${ }^{\mathrm{e}}$, Daniel Müller ${ }^{\mathrm{c}}$, Kripa K. Varanasi ${ }^{\mathrm{d}}$, Frank Muecklich ${ }^{\mathrm{c}}$, Louis Stodieck ${ }^{\mathrm{a}, 1}$

a BioServe Space Technologies, Aerospace Engineering Sciences Department, University of Colorado, Boulder, 80309, USA

${ }^{\mathrm{b}}$ Institute of Aerospace Medicine, German Aerospace Center (DLR), Cologne, 51147, Germany

${ }^{\mathrm{c}}$ Functional Materials, Department of Materials Science and Engineering, Saarland University, 66123, Germany

d Department of Mechanical Engineering, Massachusetts Institute of Technology, Cambridge, MA, 02139, USA

e Aerospace Engineering Sciences Department, University of Colorado, Boulder, 80309, USA

\section{A R T I C L E I N F O}

\section{Keywords:}

Bacteria

Fungi

Pseudomonas aeruginos

Penicillium rubens

Direct laser-interference patterning (DLIP)

Lubricant-impregnated surface (LIS)

\begin{abstract}
A B S T R A C T
Biofilm growth has been observed in Soviet/Russian (Salyuts and Mir), American (Skylab), and International (ISS) Space Stations, sometimes jeopardizing key equipment like spacesuits, water recycling units, radiators, and navigation windows. Biofilm formation also increases the risk of human illnesses and therefore needs to be well understood to enable safe, long-duration, human space missions. Here, the design of a NASA-supported biofilm in space project is reported. This new project aims to characterize biofilm inside the International Space Station in a controlled fashion, assessing changes in mass, thickness, and morphology. The space-based experiment also aims at elucidating the biomechanical and transcriptomic mechanisms involved in the formation of a "columnand-canopy" biofilm architecture that has previously been observed in space. To search for potential solutions, different materials and surface topologies will be used as the substrata for microbial growth. The adhesion of bacteria to surfaces and therefore the initial biofilm formation is strongly governed by topographical surface features of about the bacterial scale. Thus, using Direct Laser-Interference Patterning, some material coupons will have surface patterns with periodicities equal, above or below the size of bacteria. Additionally, a novel lubricant-impregnated surface will be assessed for potential Earth and spaceflight anti-biofilm applications. This paper describes the current experiment design including microbial strains and substrata materials and nanotopographies being considered, constraints and limitations that arise from performing experiments in space, and the next steps needed to mature the design to be spaceflight-ready.
\end{abstract}

\section{Introduction}

\subsection{Biofilms}

Biofilms are formed by groups of organisms that are adhered to each other by self-synthesized extracellular polymeric substances, and are ubiquitous in industrial and natural environments [1]. The formation of biofilms increases the risk of pathogen transmission in food handling facilities, drinking water systems, and medical devices. Furthermore, biofilms can decrease the efficiency and lifetime of equipment such as heat exchangers, air and water recycling systems, etc. [1,2]. Biofilm bacteria and fungi tend to have an increased resistance to disinfectants, antibiotics, and environmental stresses - such as salt, oxidizers, and low $\mathrm{pH}-$ making it difficult to address the problems that arise from their formation [3-5]. In addition to the challenges that emerge from their formation on surfaces, biofilms play an important role in several human diseases and infections, including endocarditis (bacterial infection of cardiac tissue), cystitis (a urinary tract infection), and otitis media (an inflammatory disease of the middle ear) [3], to name a few.

\subsection{Biofilms in space}

Microbial contamination was observed on piping and equipment behind panels on board Salyut 6; water recycling system, rubber of hatch locks, electrical connectors, and the thermal control system's radiator on board Salyut 7; and air conditioning, oxygen electrolysis block, EVA suit's headphone, water recycling unit, and thermal control system on board Mir [6]. Bacillus polymira, Penicillium chrysogenum

\footnotetext{
" Corresponding author.

E-mail address: Luis.Zea@Colorado.edu (L. Zea).

${ }^{1}$ This works was originally presented at the 68th International Astronautical Congress, Adelaide, Australia in 2017 IAC-17.A1.6.8x36309.

https://doi.org/10.1016/j.actaastro.2018.04.039

Received 20 December 2017; Received in revised form 19 April 2018; Accepted 21 April 2018

Available online 23 April 2018

0094-5765/ (c) 2018 The Authors. Published by Elsevier Ltd on behalf of IAA. This is an open access article under the CC BY-NC-ND license

(http://creativecommons.org/licenses/BY-NC-ND/4.0/).
} 
(now called $P$. rubens), and Aspergillus sp. were determined to be responsible for progressive destruction of a navigation window on board Mir (reviewed in Ref. [6]). An assessment of the microbes living in the Russian Mir space station revealed 234 different species of bacteria and fungi. Most of the isolated fungi were potential biodegradors of polymers, and thus presented a potential hazard to structural materials and components of several systems [7]. Novikova et al. [8] performed a sixyear study aimed at characterizing the microbiome present on board ISS, and found that bacterial concentrations of up to $1 \times 10^{2} \mathrm{CFU} / \mathrm{mL}$ were found in potable water with Sphingomonas sp. and Methylobacterium $\mathrm{sp}$. being the dominant genera (same as the $100 \mathrm{CFU} / \mathrm{mL}$ limit described on the ISS' Medical Operations Requirements Documents (MORD) [9]). Airborne bacteria were quantified to be as high as $7 \times 10^{2} \mathrm{CFU} / \mathrm{m}^{3}$ (the ISS MORD's limit is $1 \times 10^{3} \mathrm{CFU} / \mathrm{m}^{3}$ ) Staphylococcus sp. being the dominant genus. Samples collected from surfaces showed bacterial concentrations as high as $4 \times 10^{4} \mathrm{CFU} / 100 \mathrm{~cm}^{2}$ (Staphylococcus sp.) (MORD limit: $1 \times 10^{4} \mathrm{CFU} / 100 \mathrm{~cm}^{2}$ ) and fungal concentrations of up to $3 \times 10^{5} \mathrm{CFU} / 100 \mathrm{~cm}^{2}$ (Aspergillus sp. and Cladosporium sp.) (MORD limit: $1 \times 10^{2} \mathrm{CFU} / 100 \mathrm{~cm}^{2}$ ), where the dominant genera are indicated in parenthesis. The organic acids synthesized by microorganisms can degrade metallic surfaces, which can lead to hardware malfunctioning and short circuits. Checinska et al. [10] have reported more recent results about the ISS microbiome and concluded that Actinobacteria was the most common phylum on the space station. Recently, more profound investigations showed that the microbe biodiversity of the ISS was distinct to the one from the Spacecraft Assembly Facility (SAF) cleanrooms at JPL, suggesting the human skin-associated microbes play an important role on spacecraft microbial diversity [10-12]. This has also been corroborated via a metagenome profile study [13]. Figs. 1 and 2 show biofilm formation on different components used for water processing on board ISS, taken after their return to Earth. Fig. 1 shows how only one of 12 channels was not blocked by biofilm.

\subsection{Biofilm investigations conducted in space}

At least three investigations have been conducted in space to assess biofilm formation (Table 1). The first of these experiments, reported by Pyle et al. [2], took place in the European Space Agency's PHORBOL cassettes hardware on board the Space Shuttle STS-81 mission. Burkholderia cepacia isolated from a Space Shuttle water system was used as the model organism. In this experiment, samples were grown in sterile

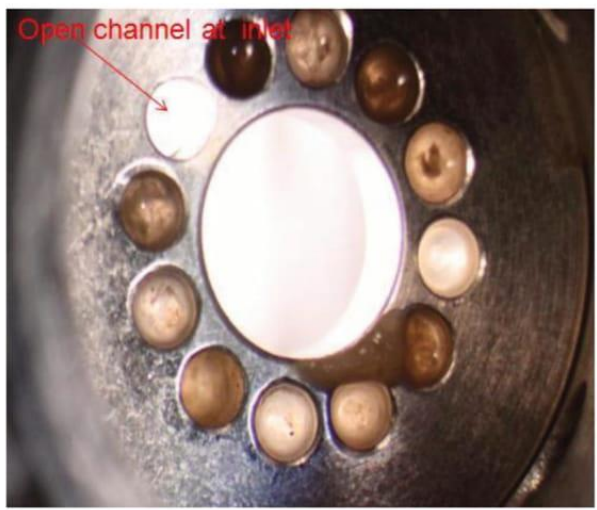

Fig. 1. Biofilm formation inside the solenoid valve at the inlet to the International Space Station's Water Processor Mostly Liquid Separator (immediately downstream of the Water Processor Waste Tank). The image was taken by United Technologies Aerospace Systems (UTAS) during disassembly of the valve at the supplier facility (ValveTech, Inc) after the valve's return to Earth. Image: NASA.

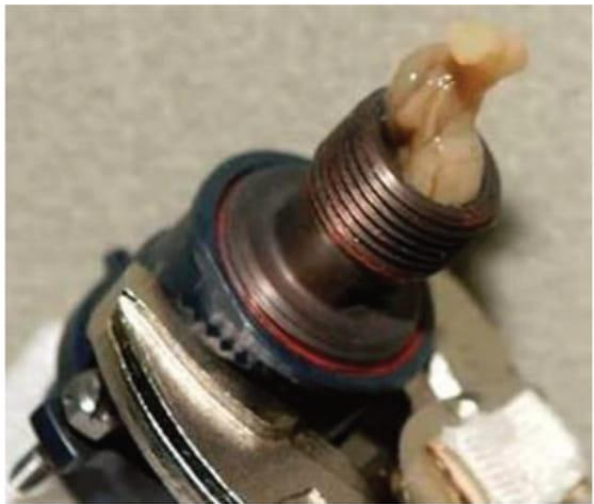

Fig. 2. Biofilm formation inside the condensate plumbing at the inlet to the Russian condensate processor. Image: NASA.

reagent grade water (to simulate untreated water), tryptic soy broth (TSB) (to simulate wastewater), or an iodine solution (to simulate disinfected potable water), and exposed for six days to a stainless-steel coupon to allow for biofilm formation. The results showed that the spaceflight water-grown bacteria had a biofilm plate count $\left(\mathrm{CFU} / \mathrm{cm}^{2}\right)$ five times that of the Earth controls. On the other hand, the spaceflight TSB-grown culture biofilm population was one quarter of the ground controls. Analyses of the water- and iodine-grown planktonic bacteria showed a 3.5- and 2-fold increase in $\mathrm{CFU} / \mathrm{mL}$ with respect to matched ground controls, respectively. Pyle et al. [2] concluded that spaceflight enhanced bacterial growth and diminished disinfectant (iodine) sensitivity in some conditions. Additionally, elongated cells and chains of cells were observed on the spaceflight samples with respect to their matched ground controls, especially on the sets grown in the iodine solution.

The next experiment used Instrumentation Technology Associates, Inc.'s Type III Osmotic Dewatering hardware to assess if Pseudomonas aeruginosa could form biofilms in microgravity. Bacterial cultures were exposed to $0.2 \mu \mathrm{m}$ polycarbonate membranes, allowing them to form biofilms for either 1 or 8 days. Post-flight confocal laser scanning microscopy revealed that biofilms indeed formed during spaceflight but no differences were observed between space and ground samples in terms of morphology. Likely due to the limitations of spaceflight experimentation, this experiment consisted of four spaceflight cultures, of which two were used for microscopic analysis [4].

The most complete and systematic spaceflight biofilm investigation conducted thus far was reported by Kim et al. [14]. Two experiments were conducted using BioServe Space Technologies' Fluid Processing Apparatus (FPA) during the STS-132 and -135 missions, with three strains of $P$. aeruginosa used as model organisms. The experiments assessed the role that phosphate, carbon source, bacterial motility, and oxygen availability play in biofilm formation in space. It was concluded that the number of viable cells, biomass and mean biofilm thickness was increased in space, regardless of phosphate concentration or carbon source. Interestingly, biofilms formed in space exhibited a "columnand-canopy" structure as opposed to the flat structures observed on the ground controls. However, this was only true for the biofilms formed by motile bacteria, as non-motile strains produced flat structures similar to those seen on the Earth samples. Additionally, it was concluded that oxygen availability diminished the differences observed between microgravity and $1 \mathrm{~g}$ samples.

A low-shear modelled microgravity (LSMMG) experiment showed that, under the simulated microgravity environment, Escherichia coli formed a thicker biofilm and was more resistant to stressors - salt, ethanol and two antibiotics [3]. Separately, we observed an enhancement of planktonic cell aggregation during the Antibiotic Effectiveness 
Table 1

Previous biofilm investigations conducted in space.

\begin{tabular}{|c|c|c|c|c|c|c|c|}
\hline Year & Mission & Bacterial Species & Strain & Growth Medium & Biofilm growth substratum & Experiment duration & Ref. \\
\hline 1997 & STS-81 & Burkholderia cepacia & $\begin{array}{l}\text { Isolated from Shuttle water } \\
\text { system }\end{array}$ & $\begin{array}{l}\text { a) water } \\
\text { b) tryptic soy broth } \\
\text { c) iodine solution }\end{array}$ & Stainless steel & 6 days & [2] \\
\hline 1998 & STS-95 & $\begin{array}{l}\text { Pseudomonas } \\
\text { aeruginosa }\end{array}$ & PAO-1 & R2A broth & $\begin{array}{l}\text { polycarbonate membrane }(0.2-\mu \mathrm{m} \text { pore } \\
\text { size) }\end{array}$ & 1 day, 8 days & {$[4]$} \\
\hline $\begin{array}{l}2010 \\
2011\end{array}$ & $\begin{array}{l}\text { STS-132 } \\
\text { STS-135 }\end{array}$ & $\begin{array}{l}\text { Pseudomonas } \\
\text { aeruginosa }\end{array}$ & $\begin{array}{l}\text { PA14 (WT, } \triangle \text { motABCD, } \\
\triangle p i l B \text { ) }\end{array}$ & $\begin{array}{l}\text { Modified artificial urine } \\
\text { media (mAUM) }\end{array}$ & $\begin{array}{l}13 \mathrm{~mm} \text { Millipore mixed cellulose ester } \\
\text { membrane disc }\end{array}$ & 3 days & [14] \\
\hline
\end{tabular}

in Space (AES-1) experiment conducted on board ISS [15,16] (similar to the observations reported in Wilson et al. [17]), which occurred in parallel to differential gene expression indicating cells in space were under higher levels of stress than matched Earth controls [18]. More recently, we also reported an activation of drug-resistance mechanisms in space as a result of these microgravity-derived stresses [19].

\section{Scientific aims}

In order to help determine the physical mechanisms of material/ microorganism interaction in biofilms in space, this NASA-funded experiment aims to characterize biofilm mass, thickness, morphology, and the associated gene expression using various spaceflight-relevant microbial species and substrata materials. Additionally, this experiment has the aim of elucidating the biomechanical and transcriptomic mechanisms involved in the formation of the "column-and-canopy" biofilm architecture observed in space. Additional samples will allow for characterization of potential changes of the parameters being studied and on the genes that confer microorganisms with resistance to oxidative stress, acidity, and antimicrobials. This project also aims to investigate the role of material surface topology on biofilm formation, as well as to test a novel lubricant-impregnated surface, which may be used in the future to replace the current materials on biofilm-prone spacecraft components.

\section{Preliminary spaceflight experiment design}

The independent variables that drive the spaceflight experiment design are (i) gravity, (ii) microbial strains, (iii) substrata material/topographies, and (iv) time. Controlled parameters include temperature, growth medium, and hardware. The dependent variables are (i) biofilm mass, (ii) biofilm thickness, (iii) biofilm morphology (structure), and (iv) gene expression. Earth controls will replicate flight samples and are planned to be performed asynchronously to replicate crew operations and temperature profiles as closely as possible.

\subsection{Approach to independent variables}

3.1.1. Gravity

Gravity will have two values, microgravity on ISS (with the caveat of vibrations that occur on the Station) and $1 \mathrm{~g}$ on Earth as the control. Two microbes (one bacterial and one fungal) are being considered for this experiment - although the final selection for spaceflight requires finishing Ground Testing: P. aeruginosa PA01 and Penicillium rubens $\operatorname{ATCC}^{\circ} 28089^{\mathrm{TM}}$.

\subsubsection{Microbial strains}

$P$. aeruginosa is a gram-negative bacterium commonly found in manmade environments. It is an opportunistic and nosocomial pathogen that infects the airway, urinary tract, burns, wounds; and may also cause blood infections [20,21]. P. aeruginosa has been the model organism of at least 13 different space missions [4,14,22-25] and was collected post-flight from the Apollo 13 through 17 crews [26]. The use of this bacterial species represents a continuation of the biofilm investigations conducted in STS-95, -132, and - 135 [4,14]. More specifically, PA01 (ATCC ${ }^{\circ}$ BAA-47 ${ }^{\mathrm{TM}}$, HER-1018 [PA01]) was the $P$. aeruginosa strain chosen for consideration for this experiment because it has been fully sequenced [27] and because it has been used in a previous spaceflight study from which proteomic and transcriptomic data (space vs. Earth) has been published [22].

Infections from P. chrysogenum (now called P. rubens as explained below) are primarily encountered among immunosuppressed people [28], which is a common case for astronauts [29]. On Earth, this fungus has been responsible for cases of endophthalmitis (inflammation of the internal coats of the eye) [30], fatal necrotizing esophagitis [28], invasive pulmonary mycosis (infections in the lungs) [31], among other types of infections [32]. $P$. chrysogenum was determined to be partly responsible for progressive destruction of a navigation window on board Mir [6]. The genome of P. chrysogenum Wisconsin 54-1255 has been fully sequenced [33] and later, based on a molecular phylogenetic study, $P$. chrysogenum was reclassified as Penicillium rubens [34]. Hence, P. rubens (ATCC $^{\circ} 28089^{\mathrm{rm}}$, a. k.a WIS 54-1255 [Wisconsin 54-1255; Wis. 51-20; Wis. 48-70; NRRL, 1951; ATCC 9480]) was selected for consideration to be the fungal model organisms of the spaceflight experiment. However, the final selection is dependent on finishing Ground Testing to ensure compatibility and compliance with the constraints and requirements derived from space-based research.

\subsubsection{Substrata materials}

Eight different materials, and three of these in three different topographies, each, are being tested for potential inclusion into the finalized experiment design. These materials were pre-selected mostly based on their relevance to (i) internal and external spacecraft structures, but also to (ii) space biology research, and (iii) nosocomial infections. Coupons of $1 \mathrm{~cm} \times 1 \mathrm{~cm}$ and $1 \mathrm{~mm}$ thickness were prepared from each candidate material. The eight materials being assessed are:

3.1.3.1. Cellulose membrane. Following on the research line started by Dr. Collins et al. (reported in Ref. [14]), biofilm growth on cellulose membrane will be assessed. The same membrane that was utilized on the STS-132 and -135 experiments (hydrophilic mixed cellulose $0.22 \mu \mathrm{m}$ pore size membrane (Cat No. GSWP01300, Millipore, Billerica, MA)) will be used. In case the "column-and-canopy" structure is a feature unique to this type of substratum topography (it hasn't yet been proven otherwise) this material would be used as control and to help confirm if the previous spaceflight observations can be replicated.

3.1.3.2. Aluminum 6061. This is a material used in spacecraft structures, thermal control and cryogenic fuel systems, structures for electronic devices, panels, etc., making it a ubiquitous material in spacecraft [35-39]. According to NASA's MAPTIS material database [10024], Al6061 is a non-flammable, non-toxic material.

3.1.3.3. Titanium Ti-6Al-4V. This titanium alloy features light specific gravity, high strength, and is ideal for high-temperature applications, making it a common selection for spacecraft structures, antennae, pressure vessels, brackets, fittings, propulsion tubing lines, and 
support tubes [40-42]. This titanium alloy is also used for implants, including cardiovascular, orthopedic, dental, craniofacial, and otorhinological [43-45].

3.1.3.4. Polycarbonate. This material's properties in terms of durability, safety, versatility and shatter resistance, make it a common go-to material for spaceflight instrumentation. This material is also commonly used for space biology research. Its use in the medical field include in renal dialysis, cardiac surgery products, and surgical instruments [46]. NASA MAPTIS categorizes it as toxicity "A", permitting to have up to 50lb in a single article [68558].

3.1.3.5. Quartz. In addition to its optical properties, quartz is used for spacecraft windows due to its capability to withstand temperature extremes (including re-entry to Earth) while working as part of the pressure shell [47,48]. However, bacterial and fungal contamination (including $P$. chrysogenum) of quartz windows have been reported, as in the case of microbial growth-driven progressive destruction of a navigational window on board the Mir space station [6]. Quartz is also used in solar panels, semiconductors, lighting, coatings, adhesives and for other applications [49].

3.1.3.6. Silicone. Due to its biocompatibility and biodurability, silicone is a material commonly used in the healthcare industry, e.g. on urological catheters, surgical incision drains, and respiratory devices [50]. Silicone is also commonly used for life sciences research devices, in electronics, and for a myriad of mechanical components (e.g. Orings). NASA's MAPTIS material database [06256] categorizes silicone as an "I" rating in terms of flammability and "A" in toxicity, indicating it is a benign material to work with.

3.1.3.7. Stainless steel 316. Stainless steel 316 (SS316) is used on rocket engine components, environmental control and life support system tanks and tubing (including for potable water), and Extravehicular Mobility Unit (EMU) elements [51-53]. It is also used as a material for surgical equipment, and implants [54,55]. According to NASA's MAPTIS material database [55747], SS316 is a non-flammable, nontoxic material.

3.1.3.8. Carbon fiber. Carbon fiber's mechanical (e.g. high tensile strength and low weight) and thermal (e.g. high temperature tolerance and low thermal expansion) properties make it a material commonly used for aeroshells and other applications in spacecraft [56-59]. The applied carbon fiber sheets are woven with epichlorohydrin and epoxy resin.

3.1.3.9. Lubricant-impregnated surfaces (LIS). Given that adhesion is the first process preceding formation of a mature biofilm, adhesion prevention is a logical strategy in designing anti-fouling materials. Some strategies to create anti-adhesion surfaces include use of very smooth materials [60], modification of chemical properties, and modulation of surface hydrophobicity [61]. Hydrophobicity in particular has been explored for anti-biofouling properties; however, the influence is non-linear in that hydrophobic surfaces may cause greater initial attachment while also allowing for larger detachment rates [60]. Design of anti-adhesive surfaces is further complicated by microbial adaptations, as microbes modify gene expression and biofilm geometry to adapt to less habitable surfaces [61].

Lubricant-impregnated surfaces (LIS) are textured surfaces impregnated with a lubricating fluid that can impart remarkable mobility to both Newtonian and non-Newtonian fluids [62] (Fig. 3). The lubricating fluid is immiscible with the product it is in contact with and can be designed to be held stably within the solid textures via capillary and intermolecular forces [63,64]. A thermodynamic framework incorporating the properties of the product, lubricant, solid, and surrounding environment allows one to design stable LIS that can

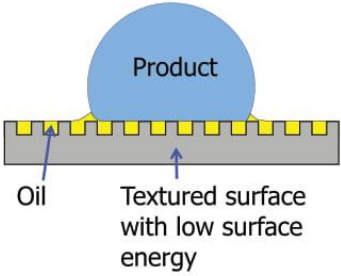

Fig. 3. Cartoon of water drop on LIS. The lubricating layer is held stable by the nanotexture and creates a boundary between the water and the solid surface.

maximize product mobility [63,64]. For example, the lubricant-solid contact angle should be below a critical angle $\theta_{C}=\cos ^{-1}(1-\varphi) /(r-\varphi)$ for the lubricant to be stably impregnated within the textures and not be displaced by the contacting medium ( $\varphi$ is the solid fraction and $r$ is roughness). It is possible to select lubricants that are biocompatible or bio-toxic, depending on the application.

LIS eliminates adhesion without relying on altered chemical/physical properties, and have been previously demonstrated for consumer products such as food and cosmetics [65], anti-icing [66] and antifouling applications $[66,67]$. Therefore, LIS is a promising candidate for anti-biofouling surfaces that microbial colonies are incapable of adapting to.

LIS are fabricated by (1) creating nano/micro scale texture, (2) imparting appropriate surface chemistry and (3) impregnation of the lubricant. Here, a random nanotexture with very low solid fraction (termed nanograss) was fabricated using reactive ion etching (Surface Technology Systems) on a typical silicone wafer. This nanotextured wafer was then diced into $10 \times 10 \mathrm{~mm}$ coupons using a laser cutter. Each coupon was cleaned using detergent, DI water, acetone, and IPA. Any remaining adsorbed organic species were then removed via plasma cleaning, and the surfaces were rendered hydrophobic using chemical vapor deposition of a flourosilane coating. Finally, silicone oil (viscosity of $100 \mathrm{cSt}$ ) was impregnated into the coupons using a dipcoater (KSV Nima) to form stable films and avoid excess lubricant $[63,68]$. Both silicone oil lubricant and silicone substrate are non-toxic and nonflammable.

\subsubsection{Substrata topographies}

The adhesion of bacteria to surfaces and therefore the initial biofilm formation is strongly governed by topographical surface features of about the bacterial scale - bacteria respond to surface topography and mechanics during the attachment phase by altering their signaling pathways between and within cells [69-71]. Thus, surface patterns at different scales (equal, above or below the size of bacteria) will be produced via Direct Laser-Interference Patterning (DLIP). The DLIP technique allows for surface patterning with lateral periodicities of about $500 \mathrm{~nm}$ to $50 \mu \mathrm{m}$ [72]. The range of bacterial size $(\sim 1-2 \mu \mathrm{m})$ is thus covered by this technique, enabling the induction of unique interactions between surface topography and bacteria or fungi.

For the DLIP, a pulsed high energy laser beam is split up in several sub-beams, which are then recombined on the materials surface, thereby creating precise intensity patterns by interference effects. Once a material is irradiated, photo-thermal interactions translate these intensity patterns into topographical surface structures. The lateral periodicity of a pattern is governed by the laser wavelength and the angle between incident laser beams, the pattern type (e.g. line- or dot-like) is determined by the number of beams. Any kind of periodic surface pattern can be generated by using an Inverse Fourier Transformation to translate the 2D-pattern into a specific 3D geometrical setup of laser beams [72]. Nanotopographies on polycarbonate, woven carbon fiber sheet and silicone coupons will be tested during Ground Tests for potential inclusion into the spaceflight experiment. 


\subsubsection{Time}

The concept of operations includes terminating the experiment by fixing the samples, either in paraformaldehyde (ACROS, Cat. No. 41678, New Jersey, USA) for morphological assessments, or in RNAProtect Bacteria (Qiagen, Cat. No. 76506, MD, USA) for gene expression analyses. The time at which samples will be fixed, as well as the appropriate fixative concentrations (fixative/sample ratios) to enable appropriate post-flight analyses, will be determined via Ground Testing.

\subsection{Controlled parameters}

Temperature and growth media will be controlled parameters. Ground Testing will determine culturing temperature but the preliminary experiment design is based on $37^{\circ} \mathrm{C}$ and $25^{\circ} \mathrm{C}$ for bacterial and fungal growth, respectively.

The growth media being considered for culturing $P$. aeruginosa include (i) sterile reagent grade water to simulate untreated water, (ii) $3 \%$ Tryptic soy broth (TSB) (as recommended by ATCC), (iii) 10\% Tryptic soy broth (TSB) to simulate wastewater, (iv) $8.8 \mathrm{mg} \mathrm{L}^{-1}$ (weak) iodine solution to simulate disinfected potable water, $(v)$ modified artificial urine media (mAUM) to simulate human urine as described in Ref. [14], (vi) LB broth, and (vii) LB broth supplemented with $5 \mathrm{~g} / \mathrm{L}$ glucose.

The growth media being considered for culturing $P$. rubens include the same first four options described for $P$. aeruginosa, plus $(v)$ potato dextrose broth (PDB), and (vi) PDB supplemented with $5 \mathrm{~g} / \mathrm{L}$ glucose.

The experiment is planned to be performed in BioServe Space Technologies' 12-Well BioCell, which is a culture system designed for space-based research. It is well-plate-sized and has customizable membranes (to change gas permeation rates), enabling for either aerobic or anaerobic growth.

\subsection{Approach to dependent variables}

Spaceflight and matched ground control samples will be fixed at experiment end. Half of the samples are planned to be fixed in paraformaldehyde (used for post-flight biofilm mass, thickness, and morphology analyses) and the other identical half will be fixed in RNAProtect Bacteria (for gene expression analyses).

\subsubsection{Biofilm mass, thickness, and morphology (structure)}

These three parameters are planned to be assessed post-flight via confocal laser scanning microscopy, calculating biomass, biofilm thickness, and structure from the three-dimensional images generated via software (e.g. Zeiss' Zen, Vaa 3D, BitPlane Imaris, NIH Image J, ImageAnalysis Comstat, or $3 \mathrm{i}$ Slidebook Reader). Samples fixed in space with PFA are planned to be stained with $1 \mu \mathrm{g} / \mathrm{mL}$ propidium iodide and $1 \%$ Triton X-100 in PBS as described in Ref. [14], although other protocols will be assessed (e.g. DAPI stain). Scanning electron microscopy imaging will also be considered for detailed cellular surface morphological analysis.

\subsubsection{Gene expression}

Gene expression data acquisition is planned to be done via RNA Sequencing and differential gene expression will be performed per [18].

\section{Preliminary microscopy analyses}

Fluorescent microscopy images are currently being used to provide preliminary insight into the material-media combinations that best enable biofilm formation, and potential differences on the biofilms' structures. Fig. 4 shows $P$. aeruginosa attached growth on quartz, after culturing in $3 \% \mathrm{TSB}$, at $37^{\circ} \mathrm{C}$ for $96 \mathrm{~h}$. Samples were fixed in $4 \%$ final PFA and stained with FilmTracer ${ }^{\mathrm{TM}}$ SYPRO ${ }^{\mathrm{mM}}$ Ruby Biofilm Matrix Stain (ThermoFisher, Cat. No. F10318) for 30 miuntes. The stain was then washed off with $\mathrm{dH}_{2} \mathrm{O}$ and stained with $10 \mu \mathrm{g} / \mathrm{mL}$ DAPI Stain

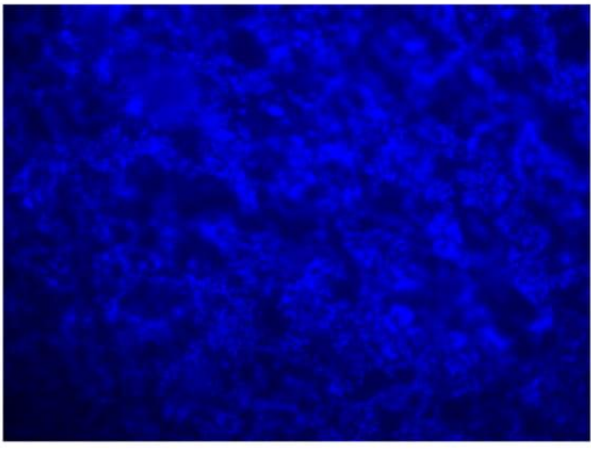

Fig. 4. P. aeruginosa biofilm formed on quartz.

(ThermoFisher, Cat. No. 62248) for $10 \mathrm{~min}$ (seen in blue in Fig. 4). The presence of cells in focus and out of focus indicate the three-dimensionality of the biofilms structure.

$P$. rubens was cultured in PDB for $96 \mathrm{~h}$ at room temperature with different material coupons. In this case, the coupons were fixed in $4 \%$ final PFA concentration and stained with Calcofluor white (CFW) and Acridine Orange (AO). CFW stains chitin (a cell wall component) blue, revealing the hyphae structure of the biofilm. AO stains doublestranded DNA green, exposing part of the overall extracellular matrix of the biofilm, which contains extracellular DNA as well as the DNA within the cells. Each coupon was stained with $10 \mu \mathrm{L}$ of $0.1 \%$ CFW plus $10 \mu \mathrm{L}$ of $1 \mathrm{mg} \mathrm{l}^{-1} \mathrm{AO}$, waiting $15 \mathrm{~min}$ in the dark [73]. Visualization of all samples ( $P$. aeruginosa and $P$. rubens) was done using the Nikon E600 Upright Wide field Microscope. Samples of $P$. rubens were cultured in BioServe's Clinostat rotated at 6 RPM; Fig. 5 shows biofilm formed on aluminum in clinorotated samples. This separate investigation will be reported in detail separately

\section{Considerations for experiment design maturation}

Space-based research generates constraints that normally do not exist in labs on Earth and that need to be considered in order to have a successful spaceflight experiment; a non-comprehensive list of these considerations is listed here. The amount of samples and kits that compose an experiment are limited by multiple factors, one being how much mass and volume can be sent to and returned from space. This usually translates into minimizing the number of samples that constitute the experimental matrix. Another consideration is temperature control for up- and down-mass, as well as in-space stowage. While manifesting items to be sent to space is difficult, it can be even more so to request them to be launched at a temperature that is not spacecraftambient. This tends to translate into performing Ground Testing, during the experiment design phase, to determine which items must be temperature controlled and which can afford not to be. A third consideration is crew time, as it is a finite resource. To address this, the experiment protocol can be designed in a fashion that minimizes crew interactions and required durations. Something else that needs to be taken into account is the time between experiment hand-over for integration into the launch vehicle - prior to lift-off - and experiment initiation, on orbit. Multiple events can occur between these two milestones. For example, for the AES-1 experiment, launch was delayed due to (i) cold temperatures at the spaceport, (ii) high space radiation on the spacecraft's path to ISS due to a solar flare, and (iii) an ammonia pump needing unplanned maintenance on ISS [15]. Hence, the experiment must be designed in a way that allows flexibility and tolerance within the experiment performance times. This is also true for the postexperiment phase of the mission, as sample return may also be delayed for myriad reasons. Other considerations that are not discussed here are 

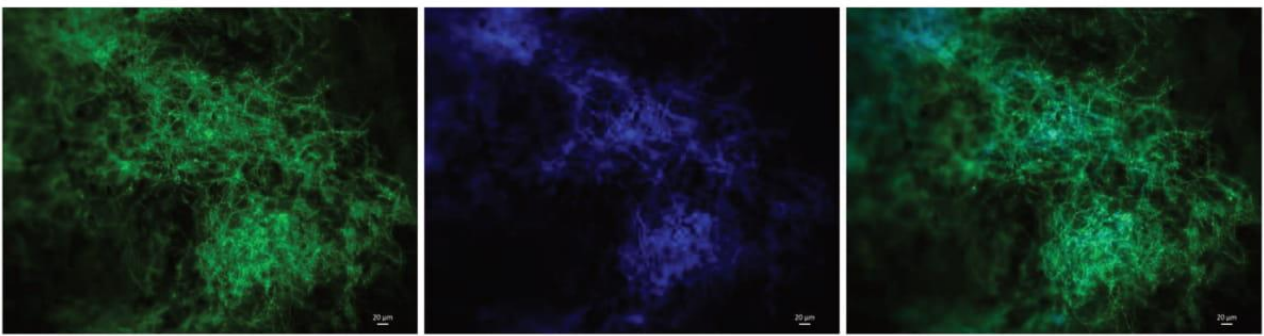

Fig. 5. Penicillium rubens biofilm attached to an aluminum coupon after $96 \mathrm{~h}$ incubation in a BioCell well, at room temperature under simulated microgravity. Left: AO reveals (in green) double-stranded DNA either within the cells or as part of the biofilm matrix as extracellular DNA. Center: CFW reveals (in blue) chitin, present in hyphae and spores cell wall, as well as in hyphal tip growth. Right: two-channel image revealing the biofilm structures. Overlapping regions appear "turquoiseblue". (For interpretation of the references to colour in this figure legend, the reader is referred to the Web version of this article.)

compliance with requirements driven by the launch/return vehicle as well as the spacecraft hosting the experiment (in this case, ISS), may they be related to safety, interfaces, compatibility, or any other type. While spaceflight-based research is now more accessible than ever, it is still far from being a routine operation.

\section{Next steps towards a spaceflight-ready experiment design}

The planned biofilm experiment described here will undergo a series of Ground Tests to inform the decisions that will enable its maturation into being spaceflight-ready. These tests include biocompatibility with the spaceflight hardware, determining the appropriate film (gas exchange rate) for microbial growth, cultivation temperature, method for adhering the material coupons onto the cell culture system without introducing a confounding factor, and characterizing the maximum durations under which experiment items (e.g. growth media, inocula, fixatives) can be stored and still be viable. Scientific and technical questions that need to be addressed include: Can the samples be launched already inoculated or do the inoculum and growth medium need to be launched separately? How long should the experiment run before fixing to enable the adequate formation of biofilms on the different material substrata being studied? What materials and nanotopographies should be included in the spaceflight experiment? For how long can the samples remain fixed and still allow for appropriate postflight analyses?

\section{Declarations of interest}

None.

\section{Acknowledgments}

This material is based upon work supported by the National Aeronautics and Space Administration under Grant No. 80NSSC17K0036. The work performed in the Moeller laboratory is supported by a grant from the German Aerospace Center (DLR grant DLR-FuW-Projekt ISS LIFE, Programm RF-FuW, Teilprogramm 475). SAM gratefully acknowledges that this work was supported by the National Science Foundation Graduate Research Fellowship Program under Grant No.1122374. The authors would like to acknowledge Chris Brown (ISS Water Operations Lead for the Flight Operations Directorate) and Layne Carter (ISS Water Subsystem Manager) for providing the images of biofilm formation in ISS components (Figs. 1 and 2) and their expert advice. LZ, ZN, PR, and MC thank Dr. James Orth for his assistance with fluorescence microscopy.

\section{References}

[1] P. Stoodley, R. Cargo, C.J. Rupp, S. Wilson, I. Klapper, Biofilm material properties as related to shear-induced deformation and detachment phenomena, J. Ind.
Microbiol. Biotechnol. 29 (6) (2002) 361-367.

[2] B.H. Pyle, G.A. McFeters, S.C. Broadaway, C.K. Johnsrud, R.T. Storga, J. Borkowski, Bacterial Growth on surfaces and in suspensions, Biorack on Spacehab. Biological Experiments on Shuttle to Mir Missions 03, 05, and 06, European Space Agency, 1999SP-1222.

[3] S.V. Lynch, K. Mukundakrishnan, M.R. Benoit, P.S. Ayyaswamy, A. Matin, Escherichia coli biofilms formed under low-shear modeled microgravity in a groundbased system, Appl. Environ. Microbiol. 72 (2006) 7701-7710.

[4] R.J.C. McLean, J.M. Cassanto, M.B. Barnes, J.H. Koo, Bacterial biofilm formation under microgravity conditions, FEMS (Fed. Eur. Microbiol. Soc.) Microbiol. Lett. 195 (2) (2001) 115-119.

[5] L. Hall-Stoodley, J.W. Costerton, P. Stoodley, Bacterial biofilms: from the natural environment to infectious diseases, Nat. Rev. Microbiol. 2 (2004) 95-108.

[6] R. Klintworth, H.J. Reher, A.N. Viktorov, D. Bohle, Biological induced corrosion of materials II: new test methods and experiences from MIR station, Acta Astronautica 44 (7) (1999) 569-578.

[7] N.D. Novikova, Review of the knowledge of microbial contamination of the Russian manned spacecraft, Microb. Ecol. 47 (2) (2004) 127-132.

[8] N. Novikova, P. De Boever, S. Poddubko, E. Deshevaya, N. Polikarpov, N. Rakova, $\mathrm{M}$. Mergeay, Survey of environmental biocontamination on board the international space station, Res. Microbiol. 157 (1) (2006) 5-12.

[9] NASA, International Space Station Medical Operations Requirements Document (ISS MORD), (2003) SSP 50260 Rev B May 2003.

[10] A. Checinska, A. Probst, P. Vaishampayan, J. White, ... K. Venkateswaran, Microbiomes of the dust particles collected from the international space station and spacecraft assembly facilities, Microbiome 3 (2015) 50.

[11] M. Mora, A. Mahnert, K. Koskinen, M.R. Pausan, L. Oberauner-Wappis, R. Krause, .. C. Moissl-Eichinger, Microorganisms in confined habitats: microbial monitoring and control of intensive care units, operating rooms, cleanrooms and the International Space Station, Front. Microbiol. 7 (2016) 1573.

[12] K. Venkateswaran, P. Vaishampayan, J. Cisneros, D.L. Pierson, S.O. Rogers, J. Perry, International Space Station environmental microbiome-microbial inventories of ISS filter debris, Appl. Microbiol. Biotechnol. 98 (14) (2014) 6453-6466.

[13] N.A. Be, A. Avila-Herrera, J.E. Allen, N. Singh, A.C. Sielaff, C. Jaing, $\mathrm{K}$. Venkateswaran, Whole metagenome profiles of particulates collected from the International Space Station, Microbiome 5 (1) (2017) 81.

[14] W. Kim, F.K. Tengra, Z. Young, J. Shong, N. Marchand, et al., Spaceflight promotes biofilm formation by Pseudomonas aeruginosa, PLoS ONE 8 (4) (2013), http://dx. doi.org/10.1371/journal.pone.0062437 e62437.

[15] L. Zea, Phenotypic and Gene Expression Responses of E. coli to Antibiotics during Spaceflight, Ph.D. Thesis University of Colorado, Boulder, 2015.

[16] L. Zea, M. Larsen, F. Estante, K. Qvortrup, R. Moeller, S. Dias de Oliveira, .. D. Klaus, Phenotypic changes exhibited by $E$. coli cultured in space, Front. Microbiol. 8 (2017) 15.

[17] J.W. Wilson, C.M. Ott, K. Honer zu Bentrup, R. Ramamurthy, L. Quick, et al., Space flight alters bacterial gene expression and virulence and reveals a role for global regulator Hfq, Proc. Natl. Acad. Sci. U. S. A. 104 (2007) 16299-16304.

[18] L. Zea, N. Prasad, S.E. Levy, L. Stodieck, A. Jones, S. Shrestha, L. Stodieck, D. Klaus, A molecular genetic basis explaining altered bacterial behavior in space, PLoS One 11 (11) (2016) e0164359.

[19] T.R. Aunins, K.E. Erickson, N. Prasad, S.E. Levy, A. Jones, S. Shrestha, ... L. Zea, Spaceflight modifies Escherichia coli gene expression in response to antibiotic ex-
posure and reveals role of oxidative stress response, Front. Microbiol. 9 (2018) 310.

[20] J.B. Lyczak, C.L. Cannon, G.B. Pier, Establishment of Pseudomonas aeruginosa infection: lessons from a versatile opportunist, Microb. Infect. 2 (9) (2000) 1051-1060.

[21] T.P. Lodise, N. Patel, A. Kwa, J. Graves, J.P. Furuno, E. Graffunder, J.C. McGregor, Predictors of 30-day mortality among patients with Pseudomonas aeruginosa bloodstream infections: impact of delayed appropriate antibiotic selec tion, AAC (Antimicrob. Agents Chemother.) 51 (10) (2007) 3510-3515.

[22] A. Crabbé, M.J. Schurr, P. Monsieurs, L. Morici, J. Schurr, J.W. Wilson, ... C.A. Nickerson, Transcriptional and proteomic responses of Pseudomonas aeruginosa $\mathrm{PAO}$ to spaceflight conditions involve Hfq regulation and reveal a role for oxygen, Appl. Environ. Microbiol. 77 (4) (2011) 1221-1230.

[23] M.A. Juergensmeyer, E.A. Juergensmeyer, J.A. Guikema, Long-term exposure to 
spaceflight conditions affects bacterial response to antibiotics, Microgravity Science and Technology 12 (1) (1999) 41-47.

[24] S.N. Zaloguyev, et al., Structural-functional changes in bacterial cells under spaceflight conditions, Dokl. Akad. Nauk SSSR 278 (5) (1984) 1236-1237 (English translation: USSR Report: Space, May 6, 1985, p.36 (JPRS-USP-85-004)).

[25] Zea, L., Stodieck, L. and Klaus, D., The first fifty years of bacterial growth and antibiotic effectiveness research in space, ASGSR Conference, Pasadena, CA, October 22-26, 2014.

[26] G.R. Taylor, Recovery of medically important microorganisms from Apollo astronauts, Aero. Med. 45 (1974) 824-828.

[27] C.K. Stover, X.Q. Pham, A.L. Erwin, S.D. Mizoguchi, Complete genome sequence of Pseudomonas aeruginosa PA01, an opportunistic pathogen, Nature 406 (6799) (2000) 959

[28] M. Hoffman, E. Bash, S.A. Berger, M. Burke, I. Yust, Fatal necrotizing esophagitis due to Penicillium chrysogenum in a patient with acquired immunodeficiency syndrome, Eur. J. Clin. Microbiol. Infect. Dis. 11 (12) (1992) 1158-1160.

[29] L.A. Mermel, Infection prevention and control during prolonged human space travel, Clin. Infect. Dis. 56 (1) (2013) 123-130, http://dx.doi.org/10.1093/cid/ cis861.

[30] M.L. Eschete, J.W. King, B.C. West, A. Oberle, Penicillium chrysogenum endophthalmitis, Mycopathologia 74 (2) (1981) 125-127.

[31] C. Geltner, C. Lass-Flörl, H. Bonatti, L. Müller, I. Stelzmüller, Invasive pulmonary mycosis due to penicillium chrysogenum: a new invasive pathogen, Transplantation 95 (4) (2013) e21-e23

[32] G. Lyratzopoulos, M. Ellis, R. Nerringer, D.W. Denning, Invasive infection due to Penicillium species other than P. marneffei, J. Infect. 45 (3) (2002) 184-195.

[33] M.A. van den Berg, R. Albang, K. Albermann, J.H. Badger, J.-M. Daran, A.J. Driessen, C. Garcia-Estrada, N.D. Fedorova, D.M. Harris, W.H.M. Heijne, V. Joarder, J.A.K.W. Kiel, A. Kovalchuk, J.F. Martín, W.C. Nierman, J.G. Nijland, J.T. Pronk, J.A. Roubos, I.J. van der Klei, N.N.M.E. van Peij, M. Veenhuis, H. von Döhren, C. Wagner, J. Wortman, R.A.L. Bovenberg, Genome sequencing and analysis of the filamentous fungus Penicillium chrysogenum, Nat. Biotechnol. 26 (2008) 1161-1168.

[34] J. Houbraken, J.C. Frisvad, R.A. Samson, Fleming's penicillin producing strain is not Penicillium chrysogenum but P. rubens, IMA fungus 2 (1) (2011) 87-95.

[35] B.J.G. Leidinger, G. Kleinert-Alvarado, R. Mueller, Design and Analysis of Spacecraft Water Evaporator (No. 901307). SAE Technical Paper, (1990).

[36] A.C. Schuerger, S. Trigwell, C.I. Calle, Use of non-thermal atmospheric plasmas to reduce the viability of Bacillus subtilis on spacecraft surfaces, Int. J. Astrobiol. 7 (01) (2008) 47-57.

[37] R. Destefanis, F. Schäfer, M. Lambert, M. Faraud, Selecting enhanced space debris shields for manned spacecraft, Int. J. Impact Eng. 33 (1) (2006) 219-230.

[38] Kumbar, P. B., Pandit, J. K., Jagadish, T., \& Thyagaraj, M. R. Enhancing the fatigue life of an electronic package in spacecraft.

[39] S. Gretchen, Fluid loop radiators for orion, Spacecraft Thermal Control Workshop. February 27-March 1, 2007, 2007

[40] N. Özdemir, B. Bilgin, Interfacial properties of diffusion bonded Ti-6Al-4V to AISI 304 stainless steel by inserting a Cu interlayer, Int. J. Adv. Manuf. Technol. 41 (5-6) (2009) 519-526.

[41] K. Miyoshi, J.H. Sanders, C.H. Hager, J.S. Zabinski, R.L. Vander Wal, R. Andrews, ... P.B. Abel, Wear behavior of low-cost, lightweight TiC/Ti-6Al-4V composite under fretting: effectiveness of solid-film lubricant counterparts, Tribol. Int. 41 (1) (2008) 24-33.

[42] S. Rawal, J. Brantley, N. Karabudak, Additive manufacturing of Ti-6Al-4V alloy components for spacecraft applications, Recent Advances in Space Technologies (RAST), 2013 6th International Conference on (pp. 5-11). IEEE, 2013, June.

[43] S.D. Cook, K.A. Thomas, J.F. Kay, M. Jarcho, Hydroxyapatite-coated titanium for orthopedic implant applications, Clin. Orthop. Relat. Res. 232 (1988) 225-243.

[44] H. Hermawan, D. Ramdan, J.R. Djuansjah, Metals for Biomedical Applications, INTECH Open Access Publisher, 2011, pp. 411-430.

[45] M. Niinomi, Recent metallic materials for biomedical applications, Metall. Mater. Trans. 33 (3) (2002) 477-486.

[46] D.G. Powell, Medical applications of polycarbonate, MEDICAL PLASTIC AND BIOMATERIALS 5 (1998) 38-45.

[47] J. Dino, NASA Ames - Shuttle Glass FAQ, (2008) Retrieved from: http://www.nasa. gov/centers/ames/research/2007/faq-shuttleglass.html.
[48] Q. Wei, H. Liu, S. He, D. Yang, Kinetics of radiation damage of quartz glass by lowenergy protons, J. Spacecraft Rockets 43 (3) (2006) 514-517.

[49] The Quartz Corporation, Quartz Applications, (2014) Retrieved from: http://www thequartzcorp.com/en/applications.html.

[50] DOW, Medical \& Healthcare Silicone Solutions, (2015) Retrieved from: http:/ www.dowcorning.com/content/discover/discovershowcase/healthcare.aspx.

[51] G.P. Sutton, O. Biblarz, Rocket Propulsion Elements, John Wiley \& Sons, 2010.

[52] L. Peterson, Environmental Control and Life Support System (ECLSS) System Engineering Workshop. ISU SSP2009, Ames Research Center, (2009) Retrieved Engineering Workshop. ISU SSP2009, Ames Research Center, (2009) Retrieved
from: http://ntrs.nasa.gov/archive/nasa/casi.ntrs.nasa.gov/20090029327.pdf.

[53] C. Campbell, Advanced EMU portable life support system (PLSS) and shuttle/ISS EMU schematics, a comparison, 42nd International Conference on Environmenta Systems, 2011, July, p. 3411.

[54] J. Oh, B. Hur, U.S. Patent No. 6,498,421, U.S. Patent and Trademark Office, Washington, DC, 2002.

[55] M.G. Bhatjiwale, A. Goel, D.P. Muzumdar, A multiposition brain holder: a versatile appliance for microneurosurgical laboratory, J. Postgrad. Med. 47 (1) (2001) 82.

[56] R. Bacon, C.T. Moses, Carbon fibers, from light bulbs to outer space, High Performance Polymers: Their Origin and Development, Springer Netherlands, 1986, pp. 341-353.

[57] M.T. Kirsch, Composite Crew Module: Primary Structure. NASA Technical Report NASA/TM-2011-217185, (2011)

[58] D. Chung, Carbon Fiber Composites. Butterworth-heinemann, ButterworthHeinemann, MA, USA, 1994.

[59] Z. Hashin, Thermoelastic properties and conductivity of carbon/carbon fiber composites, Mech. Mater. 8 (4) (1990) 293-308.

[60] S.D. Puckett, E. Taylor, T. Raimondo, T.J. Webster, The relationship between the nanostructure of titanium surfaces and bacterial attachment, Biomaterials 31 (4) (2010) 706-713.

[61] Gabriela S. Lorite, Richard Janissen, H. Clerici, Joao, Carolina M. Rodrigues, Juarez. P. Tomaz, Boris Mizaikoff, Christine Kranz, A. de Souza Alessandra, Monica A. Cotta, Surface physicochemical properties at the micro and nano length scales: role on bacterial adhesion and Xylella fastidiosa biofilm development, PLoS One 8 (9) (2013).

[62] B.R. Solomon, S.B. Subramanyam, T.A. Farnham, K.S. Khalil, S. Anand, K.K. Varanasi, Non-wettable Surfaces: Theory, Preparation, and Applications. Chapter 10: Lubricant-impregnated Surfaces, Royal Society of Chemistry, 2017.

[63] Smith, J. D., Dhiman, R., Varanasi, K. K., Cabello, E. R., 2011, Liquid-impregnated Surfaces, Methods of Making, and Devices Incorporating the Same. US Patent 8574704 .

[64] J David Smith, Rajeev Dhiman, Sushant Anand, Ernesto Reza-Garduno, Robert E. Cohen, Gareth H. McKinley, Kripa K. Varanasi, Droplet mobility on lubricantimpregnated surfaces, Soft Matter 9 (6) (2013) 1772-1780.

[65] Smith, J. D., Dhiman, R., Paxson, A. T., Love, Christopher., Solomon, B. R., Varanasi, K. K., 2011, Self-lubricating Surfaces for Food Packaging and Food Processing Equipment. US Patent 8535779.

[66] S.B. Subramanyam, G. Azimi, K.K. Varanasi, Designing lubricant-impregnated textured surfaces to resist scale formation, Advanced Materials Interfaces 1 (2014) 1300068-1300074.

[67] A.K. Epstein, T.-S. Wong, R.A. Belisle, E.M. Boggs, J. Aizenberg, Liquid-infused structured surfaces with exceptional anti-biofouling performance, National Academy of Sciences U.S.A 109 (33) (2012) 13182-13187.

[68] J. Seiwert, C. Clanet, D. Quere, Coating of a textured solid, J. Fluid Mech. 669 (2011) 55-63.

[69] K. Edwards, A. Rutenberg, Microbial response to surface microtopography: the role of metabolism in localized mineral dissolution, Chem. Geol. 180 (2001) 19-32.

[70] A.K. Epstein, A.I. Hochbaum, P. Kim, J. Aizenberg, Control of bacterial biofilm growth on surfaces by nanostructural mechanics and geometry, Nanotechnology 22 (49) (2011) 494007.

[71] A.K. Epstein, D. Hong, P. Kim, J. Aizenberg, Biofilm attachment reduction on bioinspired, dynamic, micro-wrinkling surfaces, N. J. Phys. 15 (9) (2013) 095018.

[72] F. Mücklich, A. Lasagni, C. Daniel, Laser interference metallurgy: using interference as a tool for micro/nano structuring, Int. J. Mater. Res. 97 (2006) 1-8 (awarded with Werner-Köster-Prize 2007).

[73] L.C. Simoes, M. Simoes, N. Lima, Kinetics of biofilm formation by drinking water isolated Penicillium expansum, Biofouling 31 (2015) 349-362. 
$69^{\text {th }}$ International Astronautical Congress (IAC), Bremen, Germany, 1-5 October 2018.

Copyright (C2018 by the International Astronautical Federation (IAF). All rights reserved

IAC-18-F1.2.3

Controlling Spaceflight Fungal Biofilms: the Search for Antimicrobial Surfaces

\section{Marta Cortesao*a ${ }^{\text {a }}$ Philip Rubin ${ }^{\mathrm{b}}$, Frank Mücklich $^{\mathrm{c}}$, Christine E. Hellweg $^{\mathrm{a}}$, Louis Stodieck ${ }^{\mathrm{b}}$, David Klaus $^{\mathrm{d}}$, Ralf Moeller ${ }^{\mathrm{a}}$ and Luis Zea*b}

${ }^{a}$ Institute of Aerospace Medicine, German Aerospace Center (DLR), Cologne, Germany, marta.cortesao@dlr.de

${ }^{b}$ BioServe Space Technologies, University of Colorado, Boulder, USA, luis.zea@colorado.edu

${ }^{c}$ Department of Materials Science and Engineering, Saarland University, Saarbrücken, Germany

${ }^{d}$ Aerospace Engineering Sciences, University of Colorado, Boulder, USA

* Corresponding authors

Abstract

Penicillium is one of the main fungal genera detected on board the Russian Mir Space Station and the International Space Station (ISS), demonstrating their ability to survive and maintain grow in a spaceflight environment. Filamentous fungi pose a concern to human/astronaut health because i) their airborne spores are associated with respiratory diseases; ii) their surface-associated growth promotes biodegradation of the spacecraft materials, compromising their integrity and iii) they can form biofilms associated with both water systems and medical/experimental material. To better monitor fungal contamination during long duration spaceflight missions, a NASA-funded spaceflight experiment is currently being designed to study biofilms in space. To inform the spaceflight experiment this study assessed the growth of Penicillium rubens in BioServe's 12-well BioCell, in both simulated microgravity (SMG), provided by clinorotation, and ground static control (Ground); tested its adhered growth (biofilm formation) to two different coupon materials - cellulose membrane and aluminium; and determined the impact of cold stowage $\left(-20^{\circ} \mathrm{C}\right)$ on $P$. rubens spore viability. Results reveal growth on all tested conditions in the BioCell culturing system, and biofilm formation on the tested coupons was confirmed through fluorescence microscopy. The clinorotation data suggests that $P$. rubens biofilms in space can be expected to yield equivalent mass to those of ground controls. Moreover, results show that it is possible (although not ideal) to store the spore inoculum in syringes frozen at $-20^{\circ} \mathrm{C}$ without glycerol. Most importantly, this study establishes the 12 -well BioCell as an adequate culturing system for growth of $P$. rubens in the upcoming spaceflight experiment. Besides, these prespaceflight tests represent a critical opportunity for data acquisition protocols as an initial step in the development of an effective methodology. This is of particular interest considering filamentous fungi biofilms are still not well understood, marking an important step in the study of filamentous fungi biofilms, both on Earth and in space.

Keywords: Filamentous fungi, Penicillium rubens, Biofilm, Spaceflight, Microgravity, Clinostat

\section{Acronyms/Abbreviations}

International Space Station (ISS)

Colony Forming Units (CFU)

Potato Dextrose Broth (PDB)

Potato Dextrose Agar (PDA)

Calcofluor white (CFW)

Acridine Orange (AO)

Paraformaldehyde (PFA)

Simulated microgravity (SMG)

Phosphate Buffer Saline (PBS)

Group Activation Pack (GAP)

Extra Cellular Matrix (ECM)

\section{Introduction}

The International Space Station (ISS) is home to many microorganisms, resilient enough to thrive in spaceflight conditions such as microgravity and radiation. Penicillium is one of the main fungal genera detected on board the ISS [1-4]. Among Penicillium species, Penicillium rubens has been identified aboard MIR by morphology and sequencing and has been found in ISS dust recovered from HEPA filters (350 cells $/ \mathrm{mg}$ dust) [5]. The presence of filamentous fungi in space can present both a threat and an opportunity. On one hand, $P$. rubens air borne spores can cause respiratory diseases; their surface-associated growth promotes material deterioration; and their ability to form biofilms endangers medical and experimental material, as well as industrial water systems, which have similar settings as the ones used in life-support systems on the ISS $[6,7]$. On the other hand, being the original and present-day industrial source of the antibiotic penicillin and an important producer of antifungal proteins and other relevant enzymes $[8,9]$, it provides an opportunity to adapt known 
$69^{\text {th }}$ International Astronautical Congress (IAC), Bremen, Germany, 1-5 October 2018.

Copyright $(02018$ by the International Astronautical Federation (IAF). All rights reserved.

biotechnological processes to spaceflight conditions. Biofilms are particularly threatening to astronauts' health, the integrity of materials, and water-systems sterility. Filamentous fungi biofilms have been associated with medical materials, such as prosthetics and catheters, and with human diseases where biofilms promote various infections, for example, when located in the lungs [10-12].

Despite their medical relevance, biofilms of filamentous fungi are not yet well understood, and formation models are commonly extrapolated from the known bacteria and yeast biofilms [13]. In general, they can be defined as different types of cells embedded in self-synthesized extracellular polymeric substances, describing a different phenotype when compared with planktonic cells. The most studied filamentous fungus is Aspergillus fumigatus. Recent studies of $A$. fumigatus biofilms showed up-regulation for translation, stress response, transport, cell wall/surface and secondary metabolism associated genes, and revealed increased antimicrobial resistance [14]. In A. fumigatus, an initial inoculum of $10^{5}$ spores $/ \mathrm{mL}$ was demonstrated to produce the most robust filamentous structures that are more resistant to mechanical disruption. These biofilms mature in 48-72h, with the extracellular matrix (ECM) content increasing as the biofilm matures.

Spaceflight is known to cause changes in gene and phenotypic expression, alterations in virulence, and antibiotic resistance [15-18]. P. rubens responses to simulated microgravity have been studied to some extent, demonstrating its ability to withstand growth and to produce penicillin in such conditions $[19,20]$. However, comprehensive studies on the adaptation of $P$. rubens to spaceflight conditions have not yet been performed. As long duration spaceflight missions increase in number and complexity, spacecraft integrity and astronaut health will require detailed monitoring and controlling spacecraft associated microorganisms.

To gain knowledge on fungal biofilm formation during long duration spaceflight missions, a NASAfunded project is currently being prepared [21]. In this project, a fungal and a bacterial strain will be exposed, separately, to microgravity in BioServe's 12-well BioCell culturing system aboard the ISS: the Gram-negative bacteria Pseudomonas aeruginosa and the filamentous fungus $P$. rubens. The BioCell is a 12-well-plate-sized culture chamber with customizable membranes that will house coupons of different materials. These coupons will allow to study the microbial adhesion to different surfaces which is an important part to initial biofilm formation.

This paper presents the results of part of the fungi pre-flight ground tests performed to inform the spaceflight experiment protocol. In this study, $P$. rubens was cultured in the spaceflight culture system in a clinostat to simulate certain aspects of the quiescent, low-shear extracellular environment experienced in microgravity. Growth dynamics were interrogated and compared against matched static controls. Additionally, biofilm formation on aluminum and cellulose membranes was investigated as a function of gravitational regime. Tests on how sample stowage conditions affect $P$. rubens spore viability were also performed. Results of this study are critical to inform the space experiment design, in preparation, stowage and growth conditions.

\section{Material and methods}

\subsection{Organism}

The fungal strain selected for this experiment was Penicillium rubens (formerly Penicillium chrysogenum) [22] DSM 1075 (ATCC® 28089 WIS 54-1255 [Wisconsin 54-1255; Wis. 51-20; Wis. 48-70; NRRL 1951; ATCC 9480]). This strain was selected because it was determined to be partly responsible for progressive destruction of a navigation window on board Mir [23], and because its genome has been fully sequenced [24]. Spore suspensions were harvested from 5 days-old cultures incubated in Potato Dextrose Agar (PDA) at room temperature $\left(\sim 22^{\circ} \mathrm{C}\right)$, by flooding the agar plates with Phosphate Buffer Saline (PBS) $\mathrm{pH} 7.4$ and gently scraping out the spores using a cotton stick. The spore suspensions were then filtrated through a miracloth filter (Roth), to clear hyphal fragments, and stored at $4^{\circ} \mathrm{C}$. Tittering was done using an improved Neubauer count chamber, vortexing the suspension before quantification. The same spore suspension was used to inoculate all the test wells.

\subsection{Cell culture hardware}

This study was performed using the same culture system planned to be used in the spaceflight experiment: BioServe Space Technologies' 12-Well BioCell. This BioCell is a 12-well container, developed to culture cells and organisms while abiding by the ISS's safety requirements to keep the crew and Station safe. Each of the 12 wells harbours $2.3 \mathrm{~mL}$ total volume (submerged growth). The wells are sealed with gas-permeable membranes, sufficient for cell growth, and silicone septa to enable the use of syringes with blunt cannulas for media exchange, inoculations, fixative introduction, etc. The design supports membranes made of fluorinated ethylene propylene (FEP), polycarbonate or polystyrene, among others. In this study, Type C FEP Teflon membranes were used. 
$69^{\text {th }}$ International Astronautical Congress (IAC), Bremen, Germany, 1-5 October 2018.

Copyright $(2018$ by the International Astronautical Federation (IAF). All rights reserved.

\subsection{Tested materials}

Coupon materials were selected mostly based on their relevance to (i) internal and external spacecraft structures, but also to (ii) space biology research, and (iii) nosocomial infections [21]. Coupons of $1 \mathrm{~cm} \mathrm{x} 1$ $\mathrm{cm}$, and $1 \mathrm{~mm}$ thickness were prepared from each material. The coupons were installed on the inner side of the membrane of each well using flight-proven and biocompatible double-sided tape (3M, Cat. No. 9731). The coupon materials used in this study were aluminium (Al6061) and $0.22 \mu \mathrm{m}$ cellulose membrane (Millipore, Cat. No. GSWP01300). aluminium Al6061 is non-flammable, non-toxic material used in spacecraft's structures on thermal control and cryogenic fuel systems as well as on electronic devices and panels [25-28]. The cellulose membrane was the same membrane that was utilized on the STS-132 and -135 experiments on BioServe's hardware [29]. Before placing the coupons on the BioCells, the aluminium coupons were submitted to an ultrasonic bath for $15 \mathrm{~min}$ at $30^{\circ} \mathrm{C}$ with Liqui-Nox (Alcanox, Inc) using SharperTek StaminaXP, and thoroughly rinsed with $\mathrm{dH}_{2} \mathrm{O}$

\subsection{Culturing conditions}

In total, eight 12-well BioCells were used. All conditions had each well inoculated with $10^{5}$ spores $/ \mathrm{ml}$ and were incubated in Potato Dextrose Broth (PDB) at room temperature RT of $21-22{ }^{\circ} \mathrm{C}$. The BioCells were tested in different gravitational regimes: four were incubated in simulated microgravity (SMG) whereas the other four BioCells were static, as controls. In this study, biofilm formation was tested by adding coupons of different materials into each well. These coupons allowed testing biofilm formation associated with different surfaces: aluminium and cellulose membrane. In summary, in each 12-well BioCell, four wells contained aluminium coupons; 3 wells contained cellulose membrane coupons; 5 wells had no coupons allowing for planktonic growth (as a control to elucidate if changes in biofilm formation were due to increase or decrease in planktonic cell concentrations). Coupons were place on the bottom membrane of the BioCell, and placed horizontally in the clinostat and at the table. Biomass measurement and microscopy studies were performed at two different time points: $48 \mathrm{~h}$ and $96 \mathrm{~h}$.

At each time point, cultures were fixed with $4 \%$ PFA diluted in 1x PBS, $\mathrm{pH}$ 7.4. Fixation in the BioCell wells was done by injecting double of the effective PFA concentration ( $8 \%$ PFA) onto each well and mixing the fixative solution with the culture media within the syringe until reaching the final effective fixative concentration (4\% PFA). All BioCells and well plates were put on the same table making sure that any potential clinostat motor vibrations also affected the static controls.

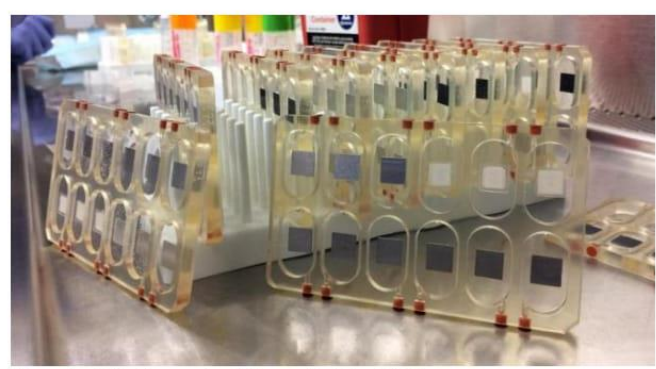

Fig. 1. BioServe's 12-well BioCells.

\subsection{Simulating microgravity}

To expose the BioCells to SMG, BioServe's clinostat was used. Microgravity was simulated by rotating the samples continuously (12 RPM) around one axis, perpendicular to the direction of the gravity vector. Under constant rotation, the particles on the BioCell experienced a constant reorientation of the gravity vector preventing cumulative sedimentation (Fig.2.). This is a functional simulation and not an exact reproduction of what happens in actual weightlessness where there is i) lack of structural deformation ii) displacement of intercellular components, and/or iii) reduced mass transfer in the extracellular fluid [30].

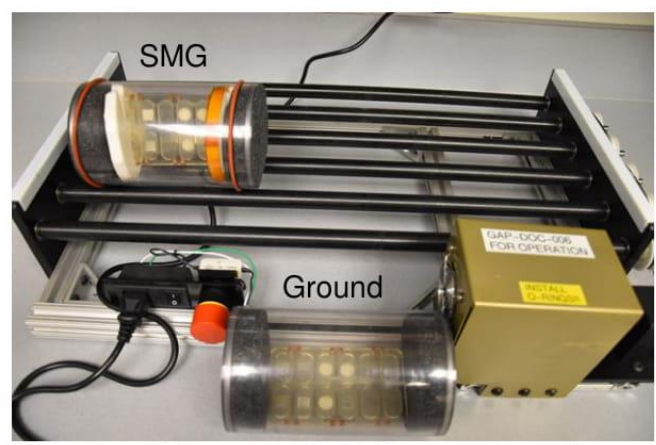

Fig. 2. BioServe's BioCells on the clinosta experiencing simulated microgravity (SMG) and respective static controls (Ground). The BioCells are placed within the Group Activation Pack (GAP). Within each well are placed the coupons: cellulose membrane and aluminium.

\subsection{Biofilm Biomass Assay}

The biofilm biomass, both planktonic and adhered, was recovered from each well and determined as dry weight. The planktonic biomass was recovered by filtration of the cultured media ( 2.3 $\mathrm{ml}$ ) using $13 \mathrm{~mm}$ PVDF syringe filters with $0.22 \mu \mathrm{m}$ 
$69^{\text {th }}$ International Astronautical Congress (IAC), Bremen, Germany, 1-5 October 2018.

Copyright $(02018$ by the International Astronautical Federation (IAF). All rights reserved.

particle retention (Fisher Scientific: Cat. No. 09-720$3)$. The filters were weighted prior to filtration. After filtration, the filter with the biomass was dried in the oven at $80{ }^{\circ} \mathrm{C}$ overnight and the dry weight was determined using a precision scale. The biomass was then calculated as "weight of the filter with biomass before filtration" minus the "dry weight of the filter before filtration".

The adhered biomass was measured by dry weight of the coupons before and after incubation. At each time point, each coupon was recovered from the respective well, after three time wash with $\mathrm{dd}_{2} \mathrm{O}$, to ensure that only coupon-associated was measured. Each coupon was dried using the same approach, and biomass was calculated as "weight of dried coupons with biomass after incubation" minus the "weight of dried coupons before incubation". Data was analysed using the program Sigma Plot. Graphs plotted using the mean and error bars represent the standard error (SE) within samples $(\mathrm{n}=3$ for cellulose membrane, $\mathrm{n}$ $=4$ for aluminum coupons; and $\mathrm{n}=4$ for no-coupon wells). Statistical analysis was done through One-way ANOVA and t-test (independent, one-tailed) where $p$ $\leq 0.05$ was considered significant.

\subsection{Fluorescence microscopy}

Staining was performed, after fixation, according to the methods described by [13]. Calcofluor White (CFW) stock concentration $1 \mathrm{mg} / \mathrm{ml}$ allows the morphological characterization of the cell walls of fungi because of its affinity for the $\beta$ (1-3) and $\beta$ (14) polysaccharides in cellulose, carboxylated polysaccharides and chitin [7]. Acridine Orange (AO) stock concentration $10 \mathrm{mg} / \mathrm{mL}$ in water (Fisher Scientific Cat. Number A3568) was used as a metabolic indicator stain, providing information on the viability of fungal biofilms. AO stains dsDNA green whereas ssDNA or RNA are stained dark orange $[7,31]$. Each microscope sample was stained with $10 \mu \mathrm{L}$ of $0.1 \% \mathrm{CFW}$ plus $10 \mu \mathrm{L}$ of $1 \mathrm{mg} / \mathrm{ml} \mathrm{AO}$ and waiting 15 minutes in the dark before visualization. Visualization of the samples was done using the Nikon E600 Upright Widefield Microscope.

\subsection{Cold stowage viability}

During pre-flight and transport to the ISS, samples will be stowed, awaiting the start of the in-flight space experiment. Ideally, BioCell samples, with media and inoculum, would be stowed at $4{ }^{\circ} \mathrm{C}$ and transported to the ISS, and temperature would be increased to $22{ }^{\circ} \mathrm{C}$, allowing for growth to start and thus marking the start of the experiment, as it is common practice in space experiments. However, $P$. rubens has demonstrated grow at $4{ }^{\circ} \mathrm{C}$ (Appendix A), thus a new option would be to send BioCells and, separately, send $3 \mathrm{cc}$ syringes with the medium and inoculum, which would be stowed at $-20{ }^{\circ} \mathrm{C}$. The astronauts would then thaw the media-inoculum and load it onto each BioCell well, in-flight, at the time of the experiment.

To determine the impact that cold stowage $(-20$ ${ }^{\circ} \mathrm{C}$ ) has on spore viability depending on the use of 50 $\%$ glycerol, several $3 \mathrm{cc}$ syringes were prepared. Each syringe harboured $2.5 \mathrm{ml}$ of the PDB medium and spore suspensions were added to a final concentration of $10^{5}$ spores $/ \mathrm{ml}$. To fill up the syringes, $50 \mathrm{ml}$ of medium was prepared in a $50 \mathrm{ml}$ falcon tube. For media with glycerol, the samples were vortexed vigorously for $1 \mathrm{~min}$, before filling the syringes. The syringes were placed inside a box and this box was stowed at $-20{ }^{\circ} \mathrm{C}$ for 10 and 20 days. After stowage time the syringes were thawed by leaving them at RT (21- $22{ }^{\circ} \mathrm{C}$ ) for $2-3$ hours. A total of $2.3 \mathrm{ml}$ of each syringe was transferred to each well of the 12-well plates for biofilm formation analysis, and the remaining volume transferred to a $1.5 \mathrm{ml}$ Eppendorf tube for further viability analysis. Viability was determined as colony forming units (CFU) after 3-4 days incubation at RT $\left(21-22{ }^{\circ} \mathrm{C}\right)$ in PDA medium supplemented with Triton-X (final concentration 0.05 $\%)$

\section{Results}

\subsection{Biofilm Biomass Assay}

To characterize growth of Penicillium rubens in the BioCell hardware, both adhered biomass (attached to the coupons) and planktonic biomass (not attached) were measured by dry weight. For wells with coupons (aluminium and cellulose membrane) total biomass corresponds to adhered + planktonic, whereas for wells with no coupons total biomass is the same as planktonic biomass.

Results show that differences in total biomass between the two time points of $48 \mathrm{~h}$ and $96 \mathrm{~h}$ were not significant. However, when assessing the effect of the gravitational regime SMG in the growth of $P$. rubens in wells with cellulose membrane coupons, a significant decrease of $19 \%$ in total biomass (adhered + planktonic) was detected, when compared with Ground controls, both at $48 \mathrm{~h}(p=0.03)$ and $96 \mathrm{~h}$ incubation $(p=0.01)$ (Fig.3.). In contrast, in wells harbouring an aluminium coupon, there were no significant differences in total biomass between the two gravitational regimes. Interestingly, in wells with no coupons, an increase of biomass (planktonic biomass) was detected when grown in SMG with respect to Ground controls, after $48 \mathrm{~h}(p=0.05)$. The same was not detected at $96 \mathrm{~h}$ (Fig.3.).

To better assess biofilm formation, the couponassociated biomass was analysed. Thus, the 
$69^{\text {th }}$ International Astronautical Congress (IAC), Bremen, Germany, 1-5 October 2018.

Copyright $\odot 2018$ by the International Astronautical Federation (IAF). All rights reserved.

percentage of adhered biomass was calculated for each coupon, having total biomass (adhered plus planktonic for wells with coupons, and only planktonic biomass for wells with no coupons) as 100 $\%$.

Results reveal an average of $93 \%$ and $97 \%$ of adhered biomass in aluminium coupons when exposed to SMG and Ground conditions, respectively, after $48 \mathrm{~h}$ of incubation; and $95 \%$ and $92 \%$ after 96 $\mathrm{h}$ of incubation. Moreover, a $3 \%$ increase in adhered biomass of aluminium coupons was identified when grown in SMG, in respect to Ground, at the $96 \mathrm{~h}$ incubation time point $(p=0.05)$. The percentage of biomass adhered to cellulose membrane coupons grown in SMG and Ground conditions was of $96 \%$ and $97 \%$ after $48 \mathrm{~h}$ and $92 \%$ and $93 \%$ after $96 \mathrm{~h}$ incubation, respectively, although with no significant differences (Fig.3.).
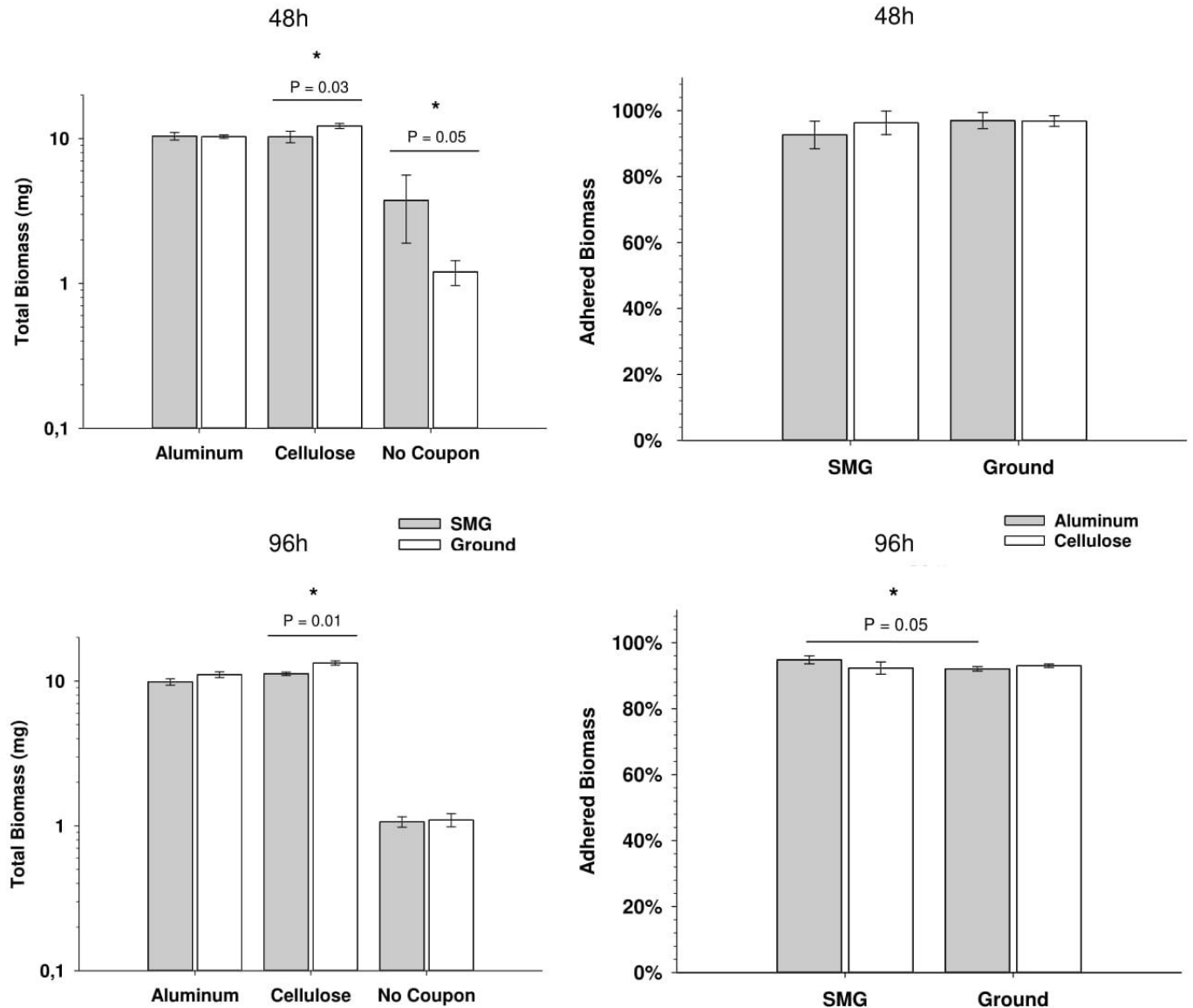

Fig. 3. On the left: Total biomass calculated as dry weight, represented by adhered and planktonic biomass for wells with coupons (aluminium and cellulose membrane), and only planktonic biomass for wells with no coupon. On the right: percentage of adhered biomass (biofilm formation) from the total biomass, for each of the tested coupons aluminium and cellulose membrane. The figure presents data from after $48 \mathrm{~h}$ (top) and $96 \mathrm{~h}$ (bottom) incubation, comparing the two gravitational regimes tested: simulated microgravity (SMG) and ground control. Error bars as standard error. $\left({ }^{*}\right) p \leq 0.05$ 
$69^{\text {th }}$ International Astronautical Congress (IAC), Bremen, Germany, 1-5 October 2018.

Copyright $(2018$ by the International Astronautical Federation (IAF). All rights reserved.

\subsection{Fluorescence microscopy}

To identify the presence of biofilm attached to the tested coupons, a qualitative assessment was performed through fluorescent microscopy. Images on Fig. 4 reveal biofilm formation on both cellulose

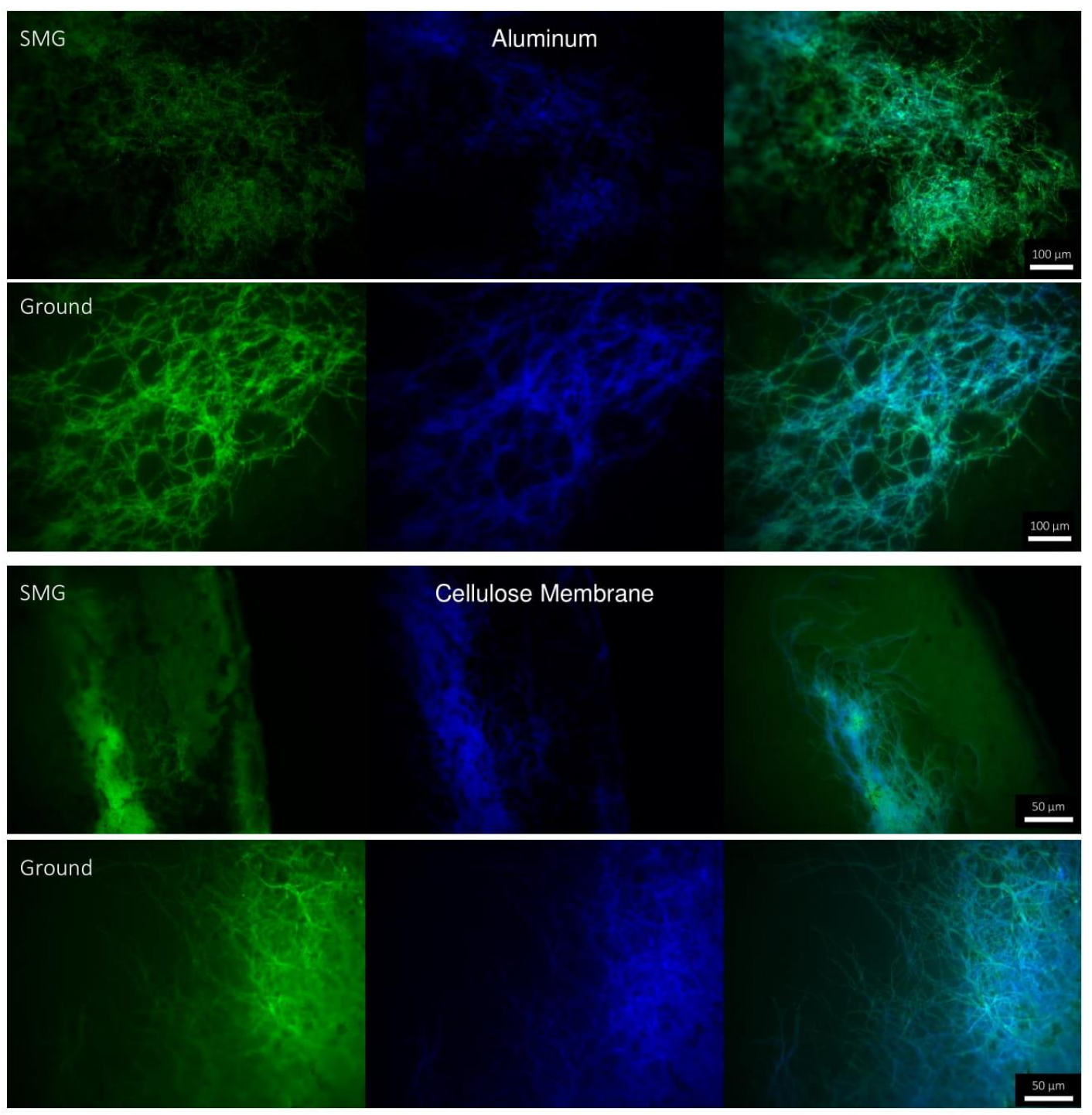

Fig. 4. Fluorescence microscopy revealing biofilm formation (adhered biomass) in aluminium and cellulose membrane coupons in the two gravitational regimes after $96 \mathrm{~h}$ incubation: simulated microgravity (SMG) and ground static control (Ground). Scale $=50 \mu \mathrm{m}$. 
$69^{\text {th }}$ International Astronautical Congress (IAC), Bremen, Germany, 1-5 October 2018.

Copyright $(2018$ by the International Astronautical Federation (IAF). All rights reserved.

membrane and aluminum coupons after $96 \mathrm{~h}$ incubation in both simulated microgravity (SMG) and the ground static controls (Ground). By staining with $\mathrm{CFW}$, the hyphal cell wall is revealed in blue, while AO stain reveals double stranded DNA (dsDNA) in green. Overlapping regions appear in turquoise blue (Fig.4.). On both coupons, an intricate hyphal network can be observed, as can be expected in a mature filamentous fungus biofilm.

\subsection{Cold stowage viability}

To test the appropriate stowage conditions, $P$. rubens spores viability was tested after 10 and 20 day stowage at $-20{ }^{\circ} \mathrm{C}$ in PDB medium, with or without $50 \%$ glycerol. Results reveal that $P$. rubens spores can maintain viability after 10 - and 20 -day stowage at $-20{ }^{\circ} \mathrm{C}$ in PDB with or without glycerol. Results also demonstrate that stowing spore inoculum in PDB and $50 \%$ glycerol leads to a general increase in viability (when compared with PDB and 0\% glycerol), although only significant after 20 days of stowage (p $\leq 0.05$ ) (Fig.5.). Visual analysis of the inoculum stowage syringes after thawing, allowed the detection of germination from fungal spores to hyphae (Appendix B).

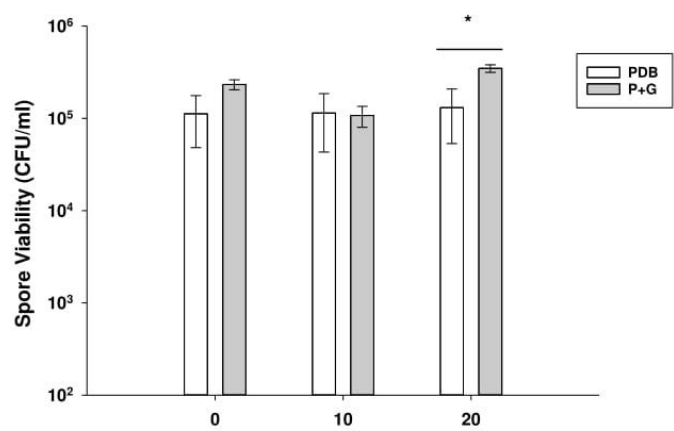

Fig. 5. Impact of cold stowage of media-inoculum, in the viability of $P$. rubens spores. Spores were kept at $20{ }^{\circ} \mathrm{C}$ for 0 -, 10 - and 20 -days, in media with $(\mathrm{P}+\mathrm{G})$ or without glycerol (PDB). Viability was calculated through CFU count. Error bars as standard deviation. ${ }^{*} p \leq 0.05$

\section{Discussion}

Fungal biofilms have been detected on board the Russian Mir Space Station and the International Space Station (ISS), posing a concern to human health and to spacecraft integrity [5]. To improve monitoring and control of fungal contamination, a
NASA-funded spaceflight experiment is currently being designed to study bacterial and fungal biofilms while also testing for antimicrobial surfaces. To inform the spaceflight experimental design a series of ground tests were conducted on BioServe's 12-Well BioCell, defining and optimizing stowage and culturing condition for the fungus $P$. rubens.

Because growth in the BioCell will inevitably differ from common laboratory containers (such as flasks or multi-well plates), it is important to assess if the fungus can grow and form biofilms; and if there is adherence to coupons and how it is compared with planktonic (non-adhered) growth. For that an initial approach tested $P$. rubens growth in the 12-well BioCell, both in simulated microgravity provided by clinorotation (SMG) and in static control (Ground), as well as its adherence to two different material coupons - cellulose membrane and aluminium. Total, adhered and planktonic biomass were measured in each well, and fluorescence microscopy of coupon attached biomass was used to identify the presence of hyphae and surrounding matrix of the fungal biofilm.

Results revealed growth and biofilm formation on the BioCell culturing system in all the conditions within $96 \mathrm{~h}$ of incubation, and coupon-attached biofilms were able to be analysed through fluorescence microscopy, after fixation with PFA. A significant $3 \%$ increase in adhered biomass of aluminium coupons when grown in SMG (compared with Ground) leads to the conclusion that biofilms formed in space are expected to be equivalent in biomass to the ones we are achieving in the lab during preliminary testing. This suggests that the spaceflight experimental protocol defined here does not require adjusting. Adhesion and biofilm formation in the material coupons was confirmed through microscopy and dried biomass measurements. Microscopy images revealing mature biofilm attached to both aluminium and cellulose Membrane coupons validate the tested experimental setting: $10^{5}$ spores $/ \mathrm{ml}$ inoculum, in 2.3 $\mathrm{ml}$ PDB media, at RT $\left(\sim 22{ }^{\circ} \mathrm{C}\right)$, for $96 \mathrm{~h}$, using BioServe's 12-well as a culturing system. Overall, this study suggests that an incubation time of $96 \mathrm{~h}$ would be enough to assess biofilm formation of $P$. rubens in this experimental setting. However, biofilm formation was not detected evenly throughout the coupons, and further time points (more than 96h incubation) should be tested. Several other coupon materials are planned for the spaceflight experiment (silicone, carbon fiber, quartz and stainless steel), so future steps will be taken towards assessing growth and biofilm formation in these coupons, in a BioCell.

When testing stowage conditions of media + inoculum, it was revealed that $P$. rubens spores can maintain viability after 10 - and 20-day stowage at -20 
$69^{\text {th }}$ International Astronautical Congress (IAC), Bremen, Germany, 1-5 October 2018.

Copyright $(2018$ by the International Astronautical Federation (IAF). All rights reserved.

${ }^{\circ} \mathrm{C}$ in PDB with or without $50 \%$ glycerol. However, the detection of spontaneous germination (from spores to hyphae) decreases the purity of the inoculum and leaves the spores/ml value to an unknown. Thus, the stowage setting here tested (spores in PDB at $-20^{\circ} \mathrm{C}$, with or without glycerol) is not ideal.

\section{Conclusions}

This study establishes the 12-well BioCell as an adequate culturing system for growth of $P$. rubens in the context of a spaceflight experiment aboard the ISS. Moreover, the clinorotation data suggests that biofilms in space can be expected to yield equivalent mass to those of ground controls. It was also found that it is possible (although not ideal) to store the spore inoculum in syringes frozen at $-20{ }^{\circ} \mathrm{C}$ without glycerol.

Besides, the performed pre-spaceflight tests represent a critical opportunity for testing data acquisition protocols as an initial step in the development of an effective methodology for fungal space microbiology. This is of particular interest considering that filamentous fungi biofilms still not well understood [14, 32, 33], marking an important step in the study of filamentous fungi biofilms, both on Earth and in space.

\section{Acknowledgements}

This study is supported by the National Aeronautics and Space Administration under Grant No. 80NSSC17K0036. The work performed in the Moeller laboratory is supported by a grant from the German Aerospace Center (DLR grant DLRFuWProjekt ISS LIFE, Programm RF-FuW, Teilprogramm 475). MC is supported by DLR/DAAD Research Fellowship - Doctoral Studies in Germany, 2017 (57370122). LZ, PR, and MC thank Dr. James Orth for his assistance with fluorescence microscopy.

\section{Appendix A (Growth of Penicillium rubens DSM} 1075 at $4^{\circ} \mathrm{C}$ in different media.)

Appendix B (Spontaneous germination of Penicillium rubens DSM 1075 spores due to stowage at $-\mathbf{2 0}^{\circ} \mathrm{C}$ without glycerol)

\section{References}

A. Checinska, A. J. Probst, P. Vaishampayan, J. R. White, D. Kumar, V. G. Stepanov, et al., Microbiomes of the dust particles collected from the International
Space Station and Spacecraft Assembly Facilities, Microbiome. 3 (2015) 50.

[2] N. D. Novikova, Review of the knowledge of microbial contamination of the Russian manned spacecraft, Microb Ecol. 47 (2004) 127-32.

[3] N. Novikova, P. De Boever, S. Poddubko, E. Deshevaya, N. Polikarpov, N. Rakova, et al., Survey of environmental biocontamination on board the International Space Station, Res Microbiol. 157 (2006) 5-12.

[4] V. M. Siqueira, H. M. Oliveira, C. Santos, R. R. Paterson, N. B. Gusmao, and N. Lima, Filamentous fungi in drinking water, particularly in relation to biofilm formation, Int J Environ Res Public Health. 8 (2011) 456-69.

[5] S. J. Vesper, W. Wong, C. M. Kuo, and D. L. Pierson, Mold species in dust from the International Space Station identified and quantified by mold-specific quantitative PCR, Res Microbiol. 159 (2008) 432-5.

[6] P. Stoodley, R. Cargo, C. J. Rupp, S Wilson, and I. Klapper, Biofilm material properties as related to shear-induced deformation and detachment phenomena, J Ind Microbiol Biotechnol. 29 (2002) 361-7.

[7] V. M. Siqueira and N. Lima, Biofilm Formation by Filamentous Fungi Recovered from a Water System, Journal of Mycology. 2013 (2013) 1-9.

[8] C. Sonderegger, L. Galgoczy, S. Garrigues, A. Fizil, A. Borics, P. Manzanares, et al., A Penicillium chrysogenum-based expression system for the production of small, cysteinerich antifungal proteins for structural and functional analyses, Microb Cell Fact. 15 (2016) 192.

[9] F.-Q. Wang, Jun Zhong, Ying Zhao, Jingfa Xiao, Jing Liu, Meng Dai, et al., Genome sequencing of high-penicillin producing industrial strain of Penicillium chrysogenum, BMC Genomics. 15 (2014).

[10] W. Eduard, Fungal spores: a critical review of the toxicological and epidemiological evidence as a basis for occupational exposure limit setting, Crit Rev Toxicol. 39 (2009) 799-864.

[11] A. L. Barcus, S. D. Burdette, and T. E Herchline, Intestinal invasion and disseminated disease associated with Penicillium chrysogenum, Ann Clin Microbiol Antimicrob. 4 (2005) 21.

[12] T. J. Walsh, E. J. Anaissie, D. W. Denning, R. Herbrecht, D. P. Kontoyiannis, K. A Marr, et al., Treatment of aspergillosis: 
$69^{\text {th }}$ International Astronautical Congress (IAC), Bremen, Germany, 1-5 October 2018.

Copyright $(2018$ by the International Astronautical Federation (IAF). All rights reserved.

clinical practice guidelines of the Infectious Diseases Society of America, Clin Infect Dis. 46 (2008) 327-60.

[13] L. C. Simoes, M. Simoes, and N. Lima, Kinetics of biofilm formation by drinking water isolated Penicillium expansum, Biofouling. 31 (2015) 349-62.

[14] S. Kaur and S. Singh, Biofilm formation by Aspergillus fumigatus, Med Mycol. 52 (2014) 2-9.

[15] L. Zea, M. Larsen, F. Estante, K. Qvortrup, R. Moeller, S. Dias de Oliveira, et al., Phenotypic Changes Exhibited by E. coli Cultured in Space, Front Microbiol. 8 (2017) 1598.

[16] L. Zea, N. Prasad, S. E. Levy, L. Stodieck, A. Jones, S. Shrestha, et al., A Molecular Genetic Basis Explaining Altered Bacterial Behavior in Space, PLoS One. 11 (2016) e0164359.

[17] J. A. Rosenzweig, O. Abogunde, K. Thomas, A. Lawal, Y. U. Nguyen, A. Sodipe, et al., Spaceflight and modeled microgravity effects on microbial growth and virulence, Appl Microbiol Biotechnol. 85 (2010) 88591.

[18] T. G. Hammond, L. Stodieck, H. H. Birdsall, J. Becker, P. Koenig, J. S. Hammond, et al., Effects of Microgravity on the Virulence ofSalmonellaTowardCaenorhabditis elegans, New Space. 1 (2013) 123-131.

[19] Y. Sathishkumar, C. Krishnaraj, K. Rajagopal, D. Sen, and Y. S. Lee, High throughput de novo RNA sequencing elucidates novel responses in Penicillium chrysogenum under microgravity, Bioprocess Biosyst Eng. 39 (2016) 223-31.

[20] S. Sheet, Y. Sathishkumar, A. S. Sivakumar, K. S. Shim, and Y. S. Lee, Low-shearmodeled microgravity-grown Penicillium chrysogenum-mediated biosynthesis of silver nanoparticles with enhanced antimicrobial activity and its anticancer effect in human liver cancer and fibroblast cells, Bioprocess Biosyst Eng. 40 (2017) 1529-1542.

[21] L. Zea, Z. Nisar, P. Rubin, M. Cortesão, J. Luo, S. A. McBride, et al., Design of a spaceflight biofilm experiment, Acta Astronautica.(2018).

[22] J. Houbraken, J. C. Frisvad, and R. A. Samson, Fleming's penicillin producing strain is not Penicillium chrysogenum but $\mathrm{P}$. rubens, IMA Fungus. 2 (2011) 87-95.

[23] R. Klintworth and H. J. Reher, Biological induced corrosion of materials II: new test methods and experiences from mir station, Acta Astronautica. 44 (1999) 569-578.

[24] M. A. van den Berg, R. Albang, K. Albermann, J. H. Badger, J. M. Daran, A. J. Driessen, et al., Genome sequencing and analysis of the filamentous fungus Penicillium chrysogenum, Nat Biotechnol. 26 (2008) 1161-8.

[25] B. J. G. Leidinger, G. Kleinert-Alvarado, and R. Mueller, "Design and Analysis of a Spacecraft Water Evaporator," 1990.

[26] R. Destefanis, F. Schäfer, M. Lambert, and M. Faraud, Selecting enhanced space debris shields for manned spacecraft, International Journal of Impact Engineering. 33 (2006) 219-230.

[27] A. C. Schuerger, S. Trigwell, and C. I. Calle, Use of non-thermal atmospheric plasmas to reduce the viability of Bacillus subtilis on spacecraft surfaces, International Journal of Astrobiology. 7 (2008).

[28] J. K. P. Prashant B Kumbar, T Jagadish, M $\mathrm{R}$ Thyagara, Enhancing the Fatigue Life of an Electronic Package in Spacecraft, JMSSE. 3 (2015) 190-194.

[29] W. Kim, F. K. Tengra, Z. Young, J. Shong, N. Marchand, H. K. Chan, et al., Spaceflight promotes biofilm formation by Pseudomonas aeruginosa, PLoS One. 8 (2013) e62437.

[30] R. Herranz, R. Anken, J. Boonstra, M. Braun, P. C. Christianen, M. de Geest, et al., Ground-based facilities for simulation of microgravity: organism-specific recommendations for their use, and recommended terminology, Astrobiology. 13 (2013) 1-17.

[31] M. Hassan, G. Corkidi, E. Galindo, C. Flores, and L. Serrano-Carreon, Accurate and rapid viability assessment of Trichoderma harzianum using fluorescencebased digital image analysis, Biotechnol. Bioeng. 80 (2002) 677-84.

[32] M. W. Harding, L. L. Marques, R. J. Howard, and M. E. Olson, Can filamentous fungi form biofilms?, Trends Microbiol. 17 (2009) 475-80.

[33] G. Ramage, E. Mowat, B. Jones, C. Williams, and J. Lopez-Ribot, Our current understanding of fungal biofilms, Crit. Rev. Microbiol. 35 (2009) 340-55. 
$69^{\text {th }}$ International Astronautical Congress (IAC), Bremen, Germany, 1-5 October 2018.

Copyright $(2018$ by the International Astronautical Federation (IAF). All rights reserved.

Appendix A

PDA

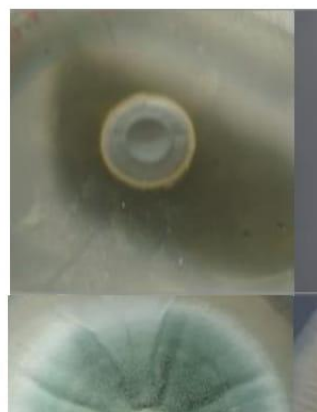

CM

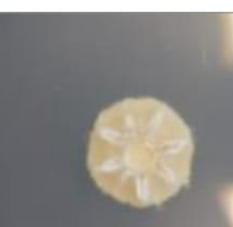

RT 22 ${ }^{\circ} \mathrm{C}$

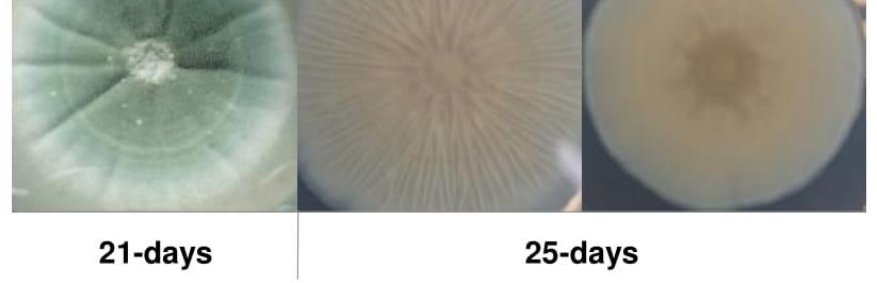

MM

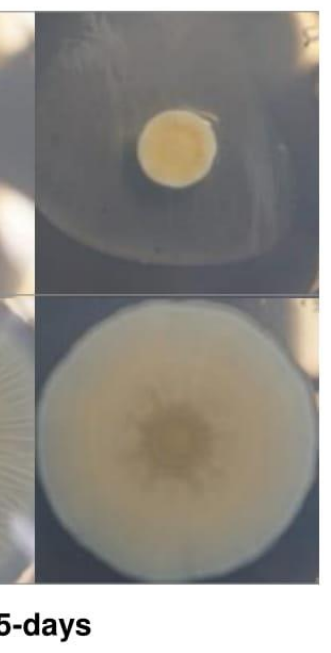

Growth of Penicillium rubens DSM 1075 at $4^{\circ} \mathrm{C}$ in different media.

PDA $=$ Potato Dextrose Agar. $\mathrm{CM}=$ Complete Medium. $\mathrm{MM}=$ Minimal Medium. MYA $=$ Malt and Yeast-extract Agar medium. RT $=$ room temperature. $P$. rubens was grown for 21 days in PDA medium, for 25 days in CM, MM and MYA media, at both $4^{\circ} \mathrm{C}$ and

\section{Appendix B}

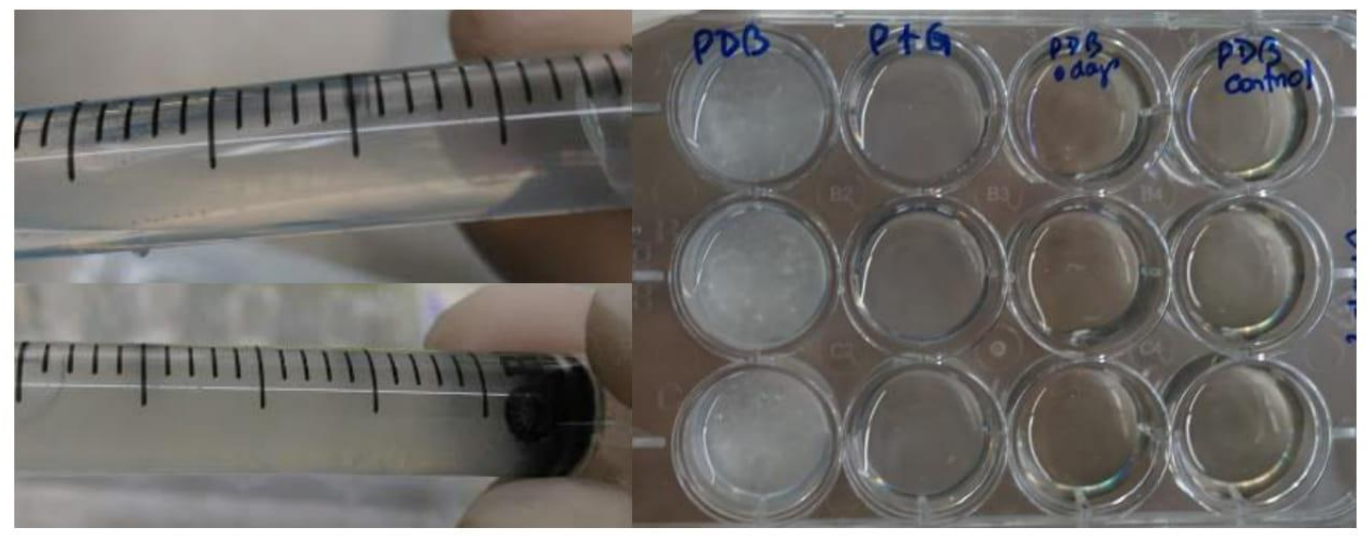

Spontaneous germination of Penicillium rubens DSM 1075 spores due to stowage at $-20{ }^{\circ} \mathrm{C}$ without glycerol On the left: syringes with spores in PDB (top) and PDB with 50\% glycerol (bottom), revealing differences in turbidity due to spontaneous germination after stowage at $-20 \mathrm{C}$ for 10 days. On the right: 12-well plate with stowage samples right after the 10 day stowage at -20C, showing spontaneous germination in PDB without glycerol (first 3 wells on the left). 


\section{Discussion}

This doctoral thesis has investigated how the filamentous fungus $A$. niger adapts to different space environments, given the fungus presence in space habitats, its medical and industrial relevance, and its role in modern-day biotechnology. For this, the industrial wild-type A. niger strain N402 (ATCC 64974) was used, as a biotechnologically relevant strain, along with three other mutant strains (derived from N402) that were defective in pigmentation $(\triangle f w n A)$, defective DNA repair via NHEJ $(\Delta k u s A)$ and with a hyperbranching phenotype $(\triangle \operatorname{rac} A)$. The resistance of $A$. niger spores to different types of space radiation was assessed by exposing wild-type, $\triangle f n w A$ and $\Delta k u s A$ spores to X-rays, cosmic rays and additionally exposing $\triangle$ racA to UV radiation. This thesis also presents investigations on the viability of $A$. niger spores in a Mars-like environment, having successfully tested the MARSBOx payload in a balloon-mission to the stratosphere, a valuable Mars-analog environment on Earth. Furthermore, in this thesis, simulated microgravity-induced changes in $A$. niger colony morphology, ultrastructure and spore physiology were identified for wild-type, $\triangle f w n A$ and $\triangle r a c A$ strains. This thesis has reported the petri-dish fast-rotating clinostat as a platform for testing filamentous fungi colony growth under simulated microgravity. Lastly, as part of this doctoral thesis, pre-flight experiments with the fungus $P$. rubens were performed in preparation for Space Biofilms - a NASA-funded spaceflight experiment aboard the ISS.

\subsection{Fungal spore resistance to space radiation}

When testing spore survival to different kinds of space radiation, it was found that spores of $A$. niger are highly resistant. Wild-type spores survived up to $1000 \mathrm{~Gy}$ of X-rays, $500 \mathrm{~Gy}$ of cosmic rays (He- and Fe-ions) and $4000 \mathrm{~J} / \mathrm{m}^{2}$ of UV-C, suggesting that they would survive in outer space, if radiation alone is considered as a stressful factor. Radiation resistance is likely due to the highly pigmented cell wall and efficient DNA repair, given that a defect in pigmentation $(\triangle f w n A$ ) or DNA repair (of the NHEJ pathway, $\triangle k u s A$ ) showed significantly less survival (to all tested types of radiation). Studies with Bacillus spores and pigmentation protection to UV radiation have found pigmentation-derived resistance only to UV-A radiation (and not to UV-B or UV-C) (Moeller et al., 2005). In contrast, in this thesis, a FwnA-derived spore pigmentation was shown to be essential in $A$. niger spore survival to UV-C radiation. In addition, melanins have been previously suggested to adsorb electromagnetic radiation such as X-rays, and were proposed as radioprotectants for space explorations (Casadevall et al., 2017; Cordero and Casadevall, 2017), however, in this thesis no difference was found in survival to X-rays between our wild-type and $\triangle$ fnwA spores.

Previous studies exposing spores of $A$. fumigatus and $A$. nidulans to up to $5400 \mathrm{~J} / \mathrm{m}^{2} \mathrm{UVB}$ found that $A$. fumigatus spores had higher frequency of cyclobutane pyrimidine dimers (CPDs) DNA photoproducts, i.e. more damage, than spores of $A$. nidulans. The authors suggested that the reduced cytoplasm space (between wall and nucleus) of $A$. fumigatus (spore size being $1 / 8$ of that of $A$. nidulans), contributed to this via low cytoplasm absorption of radiation (Nascimento et al., 2010). Despite this, knowledge about fungal conidial DNA photochemistry is largely unknown, and there is the need to identify other potential types of DNA lesions (Nascimento et al., 2010).

In this thesis, a NHEJ-defective A. niger strain ( $\triangle$ kusA, Ku70 ortholog) was also tested in exposure to space radiation. NHEJ has been implicated in a different response depending on the linear energy transfer (LET) of the radiation. High LET radiation (such as Fe-ions) is known to cause more damage, usually more double 
strand breaks, than low-LET radiation (such as X-rays or He-ions), however, strains deficient in NHEJ have shown similar survival levels at the same dose, regardless of the LET. A study with mammalian cells has determined that high-LET causes same yield of double-strand breaks, but in smaller fragments (< $40 \mathrm{bp}$ ), that don't allow Ku80 to bind and repair (Wang et al., 2008). In accordance, in this thesis it was demonstrated that the $L D_{90}$ values - i.e. dose at which there is $90 \%$ of spore inactivation - of the $A$. niger NHEJ defective strain were similar in both high-LET Fe-ions and low LET X-rays and He-ions ( 50 - 57 Gy). Other studies with $N$. crassa testing the impact of different forms of ionizing radiation found that a NHEJdeficient strain showed resistance to higher X-ray doses (low LET) than wild-type and homologous recombination-deficient strain suggesting that when defective in NHEJ, the preferential error-free HR is able to ensure DNA repair (Ma et al., 2018).

Unexpectedly upon radiation with X-rays, overnight desiccation of $A$. niger spores led to more killing of than spores radiated in wet conditions (water or saline solution). Accordingly, it has been suggested that spore killing rate during desiccation tends to increase as cellular water is removed (Dose et al., 2001), and studies with yeast cells revealed that when they are only partially dried (water content above $20 \%$ ) they begin to take up oxygen which leads to increased ROS and DNA damage (Koga et al., 1966). Furthermore, in this thesis, reduction of $A$. niger biofilm formation after UV radiation was tested. It was found that biofilm formation was reduced by $81 \%$ after treatment with $1000 \mathrm{~J} / \mathrm{m}^{2}$ of UV-C (254 nm). In long-term missions, microbial control through disinfection strategies will be critical. Approaches for UV-sterilization of water - one of the most important resources (Al-Gabr et al., 2013; Song et al., 2016) are being developed, as well as surface sterilization with plasma (ionized gas and UV radiation), both for food and medical sectors (Misra et al., 2019; Sharma et al., 2015).

\subsection{Fungal spore survival in a Mars-like environment}

In this thesis $A$. niger spore viability to a martian-like environment was studied by launching wild-type dried spores inside a Mars-gas and pressure chamber (TREX box) aboard a scientific balloon to the stratosphere (MARSBOx mission). Results suggested that $A$. niger spores might be considered some of the most likely forward contaminants to survive if inadvertently delivered to Mars. Furthermore, results also emphasize the importance of testing microbial survival towards multi-factorial Mars simulation, and the use of stratosphere as valuable Mars analog for space microbiology. Spores of $A$. niger were able to survive 5 hours of Mars-like environment, even when exposed to over $1000 \mathrm{~kJ}$ of UV (UV-A and UV-B) as a spore monolayer. Measurements aboard the balloon payload reveal ionizing radiation dose rate in the middle stratosphere ( $38 \mathrm{~km}$ altitude) to be $75.5 \mathrm{~Gy}$ per day, and further data on UV-C dose rate in the stratosphere reveal a dose rate of $1 \mu \mathrm{W} / \mathrm{cm}^{2}$. This means that on the 5-hour balloon mission, $A$. niger spores were additionally exposed to $21 \mu \mathrm{Gy}$ of ionizing radiation throughout the MARSBOx mission and to $180 \mathrm{~J} / \mathrm{m}^{2}$ of UV-C radiation. Studies with $B$. subtilis spore survival to simulated Mars conditions, have determined that spore killing is mostly UV driven, and that the spores' outer coat was the most important factor in surviving UV irradiated Mars surface conditions (Cortesão et al., 2019). Thus, it is likely that the A. niger spore cell wall also plays a critical role in adapting to Mars-like conditions.

Since the mission-experiment lasted for a total of 5 months, the laboratory controls were in themselves an opportunity to study spore resistance to long-term desiccation. Here, $A$. niger spores were found to have less than 1-log reduction after 5 months desiccation in comparison with inoculum, suggesting a high 
tolerance for desiccation. A. niger spores were also found to be resistant to extreme temperature variation effect, under artificial Mars atmosphere and pressure $\left(-51^{\circ} \mathrm{C}\right.$ to $\left.21^{\circ} \mathrm{C}\right)$. Fungal spore heat resistance, in particularly if associated with high-pressure is interesting from atmospheric re-entry, in a space travel scenario. Heat resisting molds seldom include Aspergilli, however multiple species of Aspergillus have already been found to be heat resistant, being able to survive heat shocks up to of $80{ }^{\circ} \mathrm{C}$ for 60 min (Pitt and Christian, 1970; Tribst et al., 2009). Thus, fungal spore sensitivity to extreme heat should be further evaluated to better assess the forward contamination potential in Mars analog environments. Furthermore, $A$. niger spores were able to endure cell-wall stress after exposure to Mars-like conditions, via calcofluor white (CFW) and caspofungin. CFW is an inhibitor of chitin microfibril assembly that induces the cell wall integrity pathway via RmlA which is typically sufficient for cell salvage (Fiedler et al., 2014), whereas caspofunging is an antifungic that specifically targets fungal cell wall biosynthesis, and also activates MAPK response (Altwasser et al., 2015).

Given $A$. niger extreme resistance to space radiation, survival results to Mars-like conditions strongly advise revisiting of planetary protection policies. Currently, Mars forward contamination policies restrict surface bioburden of $\leq 3 \times 10^{5}$ spores for robotic lander systems that are not carrying instruments to investigate extant martian life (caterogy IVa) (COSPAR, 2020). Most of these policies are based on scientific studies that determine the limits of microbial resistance to cleaning and sterilizing agents. However, most of these studies are performed with bacterial spores such as $B$. subtilis, which have been shown to be highly resistant to various extremes of space (He et al., 2018; Nicholson et al., 2012; Onofri et al., 2012). Insights into fungal spore resistance to decontamination are known from food industry (see above, in section 6.1) but not in the planetary protection context.

Results of this thesis also provide valuable insights to the Lithopanspermia theory (Horneck et al., 2008), since it suggests the survival of fungal spores through space travel, even without the shielding effect of a spore multilayer (i.e. self-shielding). Studies regarding how other factors - e.g. shielding by regolith or rover wheels - affect survivability during space travel have been performed with $B$. subtilis (Kerney and Schuerger, 2011; Moeller et al., 2010b; Rettberg et al., 2004). Moreover, a recent study investigated survival of the black fungus Cryomyces antarcticus to accelerated helium ions within Mars regolith (Pacelli et al., 2020). Another study analyzed proteomics and metabolomics of extremophilic fungi after exposure to Mars simulated conditions. It found that ten out of thirteen tested fungal species were able to survive the UV-C dose of 2,000 J/m², but only four strains survived 5-min exposure to simulated Mars conditions, A. fumigatus isolated from ISS HEPA filter was among the survivors (Blachowicz et al., 2019b).

\subsection{Colony growth under simulated spaceflight microgravity}

Changes in $A$. niger colony growth and biofilm formation under simulated microgravity were studied using a solid media (agar) fast-clinorotation. A summary of colony and spore microgravity-induced changes can be found in Table 3. Results show that $A$. niger has increased colony area and a thicker biofilm (i.e. vegetative mycelium embedded in ECM) in the simulated microgravity environment, which suggests increased colonization ability in a real spaceflight environment. Furthermore, results suggest that fwnA a gene encoding a Putative polyketide synthase (PKS) involved in dihydroxynaphthalene (DHN) melanin is involved in adaptation to microgravity, as its absence led to changes in colony area, total biomass, mycelium thickness and spore production. Another study analyzing the proteome and secretome of the black fungus Knufia chersonesos under simulated microgravity using HARV found upregulation of scytalone 
dehydratase, an enzyme also involved in the synthesis of DNH-melanin (Tesei et al., 2021). Melanins, as secondary metabolites are known to be involved in several cellular processes from virulence to radiation protection (Cordero and Casadevall, 2017). However, the melanin biosynthesis pathway in A. niger is not yet well understood. In addition, simulated microgravity experiments in this thesis suggest that racA - a gene encoding a Rho GTPase involved in regulation of cell polarity - is also involved in the fungus adaptation to microgravity. It is important to note that the tested strain is RacA-deficient with a background deletion of kusA, and is therefore also defective in NHEJ. Given changes in colony growth, it is possible that simulated microgravity affects DNA, if so, it will most likely be in the form of indirect damage such as reactive oxygen species. However, how fungi adapt to microgravity is still far from being understood.

Most importantly, in this thesis, never before seen SEM images of $A$. niger colonies were acquired in both Ground and simulated microgravity conditions. These images reveal the complex ultrastructure of the vegetative mycelium embedded in extracellular matrix, i.e. the biofilm. Fungal biofilms are known threats to indoor habitats, medical and scientific instruments, life-support systems and human respiratory infections. What's more, fungal biofilms are known to be highly resistant to decontamination methods and antifungal treatments (Ramage et al., 2012). Fungal biofilm formation in the spaceflight environment is a challenge, not only in the ISS, but also in indoor environments on Earth (e.g. hospitals and airplanes), and will be even more challenging in upcoming long-term space missions, i.e., to the Moon, or Mars. The challenge lies mainly in ensuring the integrity of spacecraft material surfaces and in ensuring crew health. Biodegradation of spacecraft surfaces happen because, as a saprophyte, $A$. niger grows associated with surfaces by secreting enzymes that promote biodeterioration and biocorrosion (Gutarowska, 2010). These enzymes are released from the hyphae and help break down the material substrates, further digesting and absorbing the products through the cell wall. Fungal pathogens rely on these digestive enzymes to be able to penetrate natural host barriers and infect biotic surfaces, and also enables them to colonize a widerange of abiotic surfaces (Hoffmeister and Keller, 2007).

Indeed, stress resistance and pathobiology are interlinked in Aspergilli (Brown and Goldman, 2016; Hartmann et al., 2011), and therefore changes in colonizing ability, e.g. through biofilm thickness, will not only affect pathogenicity potential but also fungal resistance to decontamination and to antifungal treatments. There are many factors contributing to successful surface colonization: mainly nutrient availability, and surrounding environmental conditions. On the ISS, relative humidity and temperature are highly controlled as part of the Environmental Control and Life-Support System (ECLSS), creating an ideal habitat for humans that can also facilitate microbial growth (ESA, 2010). When it comes to nutrient availability, humans are the common providers. Similar to the fungal growth on the ISS wall panel that was enabled by the nutrients present in the sweat, other contamination scenarios are created through humanderivate nutrient sources (e.g. sweat, urine, skin, feces etc.). This happens naturally, as the human microbiome is highly complex, and these microbes are easily spreadable in an indoor-closed environment (Garrett-Bakelman et al., 2019). Thus, the presence of other microbes (dead or alive), in any given surface, can function as nutrient sources for fungi to grow. It is important to bear in mind the fact that microbial biofilms can form not only in a mono-species, but also as a multi-species. In the natural environment, multi-species biofilms are present both in biotic and abiotic surfaces, and this is no different on the ISS and its astronauts. For instance, in human infections the interaction between fungi and bacteria is known to 
affect their pathogenic potential (Peleg et al., 2010, Melloul et al., 2016) have validated an in vitro model of mixed biofilm with the fungus $A$. fumigatus in association with the bacterium Stenotrophomonas maltophilia, mimicking a respiratory multi-species biofilm infection. Here, liquid static co-cultures were established and SEM and TEM analysis found bacterial-induced changes in the fungal biofilm, namely significantly less biofilm thickness and a thicker $A$. fumigatus cell wall when grown together with $S$. maltophilia (Melloul et al., 2016). Therefore, further studies with mixed biofilms, especially considering spacecraft environmental conditions and relevant material surfaces, should be performed to have a real risk assessment for human infections and material contamination in spaceflight.

Table 3. Effect of simulated microgravity on Aspergillus niger colony growth and spore vitality

\begin{tabular}{|c|c|c|c|}
\hline & Wild-type & Pigmentation $(\Delta f w n A)$ & Hyperbranching $(\triangle r a c A)$ \\
\hline Colony Area $\left(\mathrm{cm}^{2}\right)$ & $\uparrow$ & $\uparrow$ & $\uparrow$ \\
\hline Dried biomass (mg) & $=$ & $\uparrow$ & $\downarrow$ \\
\hline Spores per colony & $\uparrow$ & $\uparrow$ & $\uparrow$ \\
\hline Spore metabolic max (\%) & $\downarrow$ & $=$ & $=$ \\
\hline Spore metabolic max (h) & $\uparrow$ & $=$ & $\downarrow$ \\
\hline Spore hydrophobicity & $=$ & $=$ & $\uparrow$ \\
\hline Biofilm thickness $(\mu \mathrm{m})$ & $\uparrow$ & $\downarrow$ & $\uparrow$ \\
\hline Conidiophore height $(\mu \mathrm{m})$ & $\downarrow$ & $\uparrow$ & $=$ \\
\hline
\end{tabular}

\subsection{Penicillium rubens as model organism for a spaceflight experiment}

Part of this thesis was done in the scope of the spaceflight experiment aboard the ISS called Space Biofilms, led by Dr. Luis Zea from Bio Serve Space Technologies and University of Colorado, Boulder. The spaceflight experiment has the main goal to study the morphological and transcriptional changes of biofilm formation on real microgravity environment. For that, two microorganisms, known biofilm formers, were included: Pseudomonas aeruginosa and Penicillium rubens. The author of this thesis, Marta Cortesão, was part of the Space Biofilms team since 2017, being responsible for the performance of preparatory tests (or preflight tests) with $P$. rubens, that would help design and establish the final space experiment. Therefore, these preparatory tests are presented as part of this doctoral thesis. Four main tests were performed: biocompatibility; growth under simulated microgravity with test materials; establishment of protocols for analysis of morphology and material-colonization (biofilm formation); as well as transport and stowage conditions to and from the space station (pre- and post-flight). These tests were critical to inform the final experimental design and contributed to the successful development of the space experiment launched in November 2020. Unfortunately, when aboard the ISS, the fungal samples were not activated due to an 
alteration of astronaut schedule. This means that fungal growth did not start and therefore the experiment was not carried out. NASA has been kind to consider a second flight where the Space Biofilms team would re-launch and re-test the fungal samples.

While testing $P$. rubens biocompatibility and growth under simulated microgravity, analysis of fungal material colonization by fluorescence microscopy revealed the ability of $P$. rubens to grow attached to an aluminium surface, and that this growth was not hindered in simulated microgravity through slowclinorotation. The tested aluminium material is commonly used as spacecraft surface in thermal and cryogenic fuel systems as well as in electronic devices and panels. In fact, Penicillium spp. have been associated with corrosion aluminium (Yang et al., 1998), and other microbes have also been identified as able to degrade aluminium on spacecraft (Rcheulishvili et al., 2020). Since fungal-led biodegradation (or biofouling) is characteristic of indoor environments, not only of spacecraft and aircraft but also of hospitals and industrial systems (Coetser and Cloete, 2005; Kokilaramani et al., 2021; McNamara et al., 2005; Mora et al., 2016), further studies regarding fungal biodegradation in spaceflight materials should be performed to help guarantee spacecraft material integrity and astronaut health. For instance, similar studies to those done in Apollo with spacesuits (Breuker et al., 2003). Moreover, studies developing novel spacecraft material surfaces with long-term antimicrobial (both antibacterial, antifungal and antiviral) properties (Hahn et al., 2017; Paton et al., 2020) will be key to ensure a successful human space exploration. 


\section{Conclusion and Outlook}

Investigating how the fungus $A$. niger adapts to space conditions, namely to simulated microgravity, radiation and simulated Mars-surface conditions, this thesis can conclude that:

- Spores of the fungus $A$. niger are highly resistant to all tested kinds of radiation (X-rays, He- and Fe-ions, and X-rays), and would survive space travel, this is, if we consider the radiation environmental factor alone. FwnA-derived pigmentation is not involved in protecting spores from ionizing radiation. In contrast, wild-type highly pigmented spores were, as expected, more resistant than pigmentation-deficient spores when exposed to UV-C radiation. Partial desiccation of $A$. niger spores leads to an unexpected lower survival to X-ray radiation.

- A. niger spores can survive Mars-like surface conditions (i.e. desiccation, temperature variation, martian atmosphere and exposure to UV-radiation) for at least 5 hours. Survival of A.niger spores was possible even as a spore monolayer (i.e. with no self-shielding), although germination was delayed in UV-exposed spores.

- A. niger colony growth in static, aerial conditions - an analog to both spacecraft material contamination and human respiratory infections - has a complex and outstanding structure revealed via SEM imaging. A. niger colony growth and spore production are not hindered in simulated microgravity. In fact, wild-type colonies have increased biofilm (i.e. ECM covered vegetative mycelium) thickness and spore production. Furthermore, FwnA-derived pigmentation, and RacA-derived polar growth are suggested to be involved in $A$. niger adaptation to microgravity.

- The fungus $P$. rubens is biocompatible with the BioCell culture system, and is able to colonize aluminum surfaces, both on ground and in simulated microgravity.

As our knowledge about fungal adaptation to spaceflight and outer space environment increases, we will be able to address the main challenges of space microbiology and space exploration. Overall, it is important to emphasize that the impact of fungal growth is not limited to the spacecraft surfaces and systems, but rather exist in complex Human-Fungi-Spacecraft interactions. First, the indoor controlled environment of space habitats creates several niches for fungi to thrive in. Second, spaceflight and mission-derived conditions set a stressful environment for astronauts that result in a compromised immune system (Crucian et al., 2018). And third, human presence was shown to impact fungal diversity of inflated lunar/martian analog habitats, where human-microbiome influences microbial potential for colonization of the different surfaces (Avila-Herrera et al., 2020; Blachowicz et al., 2017).

Thus, one challenge will be to in monitor and control fungal burden in spacecraft-associated environments. So far, most microbial monitoring in spacecraft-assembly clean rooms do not target fungi, typically integrating only 16S sequences (Moissl-Eichinger et al., 2012; Schwendner et al., 2013; Stieglmeier et al., 2012), or limit identification of fungi to culture-dependent methods (Koskinen et al., 2017). Few studies have included 18S-rRNA and ITS1-rDNA (Makimura et al., 2001). It is also important to investigate fungal resistance capabilities to extreme environments, exposing fungi to combined, and simultaneous, conditions of the space environment (e.g. radiation, vacuum, microgravity, temperature, etc.), as well as including other factors that might affect survivability (e.g. shielding from spacecraft materials or Lunar and Mars regolith). This will help inform planetary protection guidelines as well as potential space-health 
guidelines (Rettberg et al., 2016; Simões and Antunes, 2021). In the future, Moon and Mars missions will allow us to study fungi (and other microorganisms) in real extraterrestrial lunar and martian environments (i.e., partial gravity, lunar and martian regolith, radiation, etc.) (Häder, 2019; Patel et al., 2020; Turroni et al., 2020). Indeed, Mars-sample return missions such as ExoMars are already being performed, looking for extant martian life, and will bring high astrobiological value (Fairén et al., 2017; Gonzales and Stoker, 2016; Vago et al., 2017). Moreover, efforts should focus on developing fast detection of surface-degrading fungi (and other microbes) (Rcheulishvili et al., 2020) as well as on developing strategies and technologies to monitor, control and mitigate biofilms in spaceflight (Probst and Vaishampayan, 2020; Zea et al., 2020). In particular in water-systems where disinfection and waste water management are critical (Amalfitano et al., 2020; Richardson and Rautemaa-Richardson, 2019; Wong et al., 2010), and can play a role in circular bioeconomy (Collivignarelli et al., 2020; Li et al., 2019).

Lastly, there is the challenge to employ fungi as valuable components of life-support and science systems in long-term crewed space exploration. Here, fungal-based biotechnology will play a critical role, as it is an opportunity to become resource-independent, when one is far away from Earth (Cortesão et al., 2020). Studies on microbial compound production under microgravity extend our knowledge on both molecular stress resistance mechanisms and of new interesting pathways of compound production. Besides, investigations on biomining potential in microgravity and Mars gravity are currently underway, exploring the potential of geomicrobiology in space exploration (Cockell, 2010, 2020). Understanding how fungi and other microorganisms adapt to the space environment will also yield new developments is areas from as space food production, processing and packaging to textiles and construction (Meyer et al., 2020; Perchonok et al., 2012; Valero et al., 2008). Indeed, microbes are key-players in space exploration, especially in near-future Lunar and Mars settlements as they can play a critical role in life-support, biomining, terraforming, etc. (Lopez et al., 2019; Nangle et al., 2020).

As science and research development should also be sustainable in space, long-term space missions should be able to provide not only life-support system for the crew, but also a science-support system (SSS). This SSS should empower sustainable in situ, high-throughput research development in microbiology, molecular biology, synthetic biology, genetic engineering and biotechnology (Karouia et al., 2017; McNulty et al., 2021; Menezes et al., 2015), that will ensure the exploration of the microbial resources and adaptation abilities to their full-potential. One step forward was the DNA amplification and sequencing done aboard the ISS (Boguraev et al., 2017; McIntyre et al., 2016). Thus it is clear that the challenges posed by space exploration are yielding efforts to develop low-resource, sustainable and recycled science (Galli and Losch, 2019). These will likely influence how we establish science here on Earth, too. 


\section{References}

Abad, A., Fernández-Molina, J. V., Bikandi, J., Ramírez, A., Margareto, J., Sendino, J., Hernando, F. L., Pontón, J., Garaizar, J., and Rementeria, A. (2010). What makes Aspergillus fumigatus a successful pathogen? Genes and molecules involved in invasive aspergillosis. Revista Iberoamericana de Micologia, 27(4), 155-182. https://doi.org/10.1016/j.riam.2010.10.003

Acuña, M. H., Connerney, J. E. P., Wasilewski, P., Lin, R. P., Mitchell, D., Anderson, K. A., Carlson, C. W., McFadden, J., Rème, H., Mazelle, C., Vignes, D., Bauer, S. J., Cloutier, P., and Ness, N. F. (2001). Magnetic field of Mars: Summary of results from the aerobraking and mapping orbits. Journal of Geophysical Research: Planets, 106(E10), 23403-23417. https://doi.org/https://doi.org/10.1029/2000JE001404

Aerts, J. W., Riedo, A., Melton, D. J., Martini, S., Flahaut, J., Meierhenrich, U. J., Meinert, C., Myrgorodska, I., Lindner, R., and Ehrenfreund, P. (2020). Biosignature analysis of Mars soil analogs from the Atacama Desert: Challenges and implications for future missions to Mars. Astrobiology, 20(6), 766-784. https://doi.org/10.1089/ast.2019.2063

Al-Gabr, H. M., Zheng, T., and Yu, X. (2013). Inactivation of Aspergillus flavus in drinking water after treatment with UV irradiation followed by chlorination. The Science of the Total Environment, 463464, 525-529. https://doi.org/10.1016/j.scitotenv.2013.06.065

Altwasser, R., Baldin, C., Weber, J., Guthke, R., Kniemeyer, O., Brakhage, A. A., Linde, J., and Valiante, V. (2015). Network modeling reveals cross talk of MAP kinases during adaptation to caspofungin stress in Aspergillus fumigatus. PloS One, 10(9), e0136932-e0136932. https://doi.org/10.1371/journal.pone.0136932

Amalfitano, S., Levantesi, C., Copetti, D., Stefani, F., Locantore, I., Guarnieri, V., Lobascio, C., Bersani, F., Giacosa, D., Detsis, E., and Rossetti, S. (2020). Water and microbial monitoring technologies towards the near future space exploration. Water Research, 177, 115787. https://doi.org/https://doi.org/10.1016/j.watres.2020.115787

Avila-Herrera, A., Thissen, J., Urbaniak, C., Be, N. A., Smith, D. J., Karouia, F., Mehta, S., Venkateswaran, K., and Jaing, C. (2020). Crewmember microbiome may influence microbial composition of ISS habitable surfaces. PloS One, 15(4), e0231838-e0231838.

https://doi.org/10.1371/journal.pone.0231838

Azua-Bustos, A., Urrejola, C., and Vicuña, R. (2012). Life at the dry edge: Microorganisms of the Atacama Desert. FEBS Letters, 586(18), 2939-2945. https://doi.org/https://doi.org/10.1016/j.febslet.2012.07.025

Baltussen, T. J. H., Zoll, J., Verweij, P. E., and Melchers, W. J. G. (2020). Molecular mechanisms of conidial germination in Aspergillus spp. Microbiology and Molecular Biology Reviews, 84(1), e00049-19. https://doi.org/10.1128/MMBR.00049-19

Barlow, N. (Ed.). (2008). Search for life. In Mars: An introduction to its interior, surface and atmosphere (pp. 203-215). Cambridge University Press. https://doi.org/DOI: 10.1017/CBO9780511536069.009

Be, N. A., Avila-Herrera, A., Allen, J. E., Singh, N., Checinska Sielaff, A., Jaing, C., and Venkateswaran, K. (2017). Whole metagenome profiles of particulates collected from the International Space Station. Microbiome, 5(1), 81. https://doi.org/10.1186/s40168-017-0292-4

Beauvais, A., Fontaine, T., Aimanianda, V., and Latgé, J.-P. (2014). Aspergillus Cell Wall and Biofilm. 
Mycopathologia, 178(5), 371-377. https://doi.org/10.1007/s11046-014-9766-0

Beech, I. B., Sunner, J. A., and Hiraoka, K. (2005). Microbe-surface interactions in biofouling and biocorrosion processes. International Microbiology: The Official Journal of the Spanish Society for Microbiology, 8(3), 157-168.

Begum, M., Hocking, A. D., and Miskelly, D. (2009). Inactivation of food spoilage fungi by ultra violet (UVC) irradiation. International Journal of Food Microbiology, 129(1), 74-77. https://doi.org/10.1016/j.ijfoodmicro.2008.11.020

Behera, B. C. (2020). Citric acid from Aspergillus niger: a comprehensive overview. Critical Reviews in Microbiology, 46(6), 727-749. https://doi.org/10.1080/1040841X.2020.1828815

Berger, T., Burmeister, S., Matthiä, D., Przybyla, B., Reitz, G., Bilski, P., Hajek, M., Sihver, L., Szabo, J., Ambrozova, I., Vanhavere, F., Gaza, R., Semones, E., Yukihara, E. G., Benton, E. R., Uchihori, Y., Kodaira, S., Kitamura, H., and Boehme, M. (2017). DOSIS and DOSIS 3D: radiation measurements with the DOSTEL instruments onboard the Columbus Laboratory of the ISS in the years 2009-2016. J. Space Weather Space Clim., 7. https://doi.org/10.1051/swsc/2017005

Bigley, A. B., Agha, N. H., Baker, F. L., Spielmann, G., Kunz, H. E., Mylabathula, P. L., Rooney, B. V, Laughlin, M. S., Mehta, S. K., Pierson, D. L., Crucian, B. E., and Simpson, R. J. (2019). NK cell function is impaired during long-duration spaceflight. Journal of Applied Physiology (Bethesda, Md. : 1985), 126(4), 842-853. https://doi.org/10.1152/japplphysiol.00761.2018

Blachowicz, A, Mayer, T., Bashir, M., Pieber, T. R., De León, P., and Venkateswaran, K. (2017). Human presence impacts fungal diversity of inflated lunar/Mars analog habitat. Microbiome, 5(1), 62. https://doi.org/10.1186/s40168-017-0280-8

Blachowicz, Adriana, Chiang, A. J., Romsdahl, J., Kalkum, M., Wang, C. C. C., and Venkateswaran, K. (2019)a. Proteomic characterization of Aspergillus fumigatus isolated from air and surfaces of the International Space Station. Fungal Genetics and Biology : FG and B, 124, 39-46. https://doi.org/10.1016/j.fgb.2019.01.001

Blachowicz, Adriana, Chiang, A. J., Elsaesser, A., Kalkum, M., Ehrenfreund, P., Stajich, J. E., Torok, T., Wang, C. C. C., and Venkateswaran, K. (2019)b. Proteomic and metabolomic characteristics of extremophilic fungi under simulated Mars conditions. Frontiers in Microbiology, 10, 1013. https://doi.org/10.3389/fmicb.2019.01013

Blank, G., and Corrigan, D. (1995). Comparison of resistance of fungal spores to gamma and electron beam radiation. International Journal of Food Microbiology, 26(3), 269-277. https://doi.org/10.1016/0168-1605(94)00129-t

Boguraev, A.-S., Christensen, H. C., Bonneau, A. R., Pezza, J. A., Nichols, N. M., Giraldez, A. J., Gray, M. M., Wagner, B. M., Aken, J. T., Foley, K. D., Copeland, D. S., Kraves, S., and Alvarez Saavedra, E. (2017). Successful amplification of DNA aboard the International Space Station. NPJ Microgravity, 3, 26. https://doi.org/10.1038/s41526-017-0033-9

Bongomin, F., Gago, S., Oladele, R. O., and Denning, D. W. (2017). Global and multi-national prevalence of fungal diseases-estimate precision. Journal of Fungi (Basel, Switzerland), 3(4). https://doi.org/10.3390/jof3040057

Borst, A. G., and van Loon, J. J. W. A. (2008). Technology and developments for the Random Positioning Machine, RPM. Microgravity Science and Technology, 21(4), 287. https://doi.org/10.1007/s12217008-9043-2 
Braun, M., Buchen, B., and Sievers, A. (2002). Actomyosin-mediated statolith positioning in gravisensing plant cells studied in microgravity. Journal of Plant Growth Regulation, 21(2), 137-145. https://doi.org/10.1007/s003440010052

Breuker, M., McNamara, C., Young, L., Perry, T. D., Young, A., and Mitchell, R. (2003). Fungal growth on synthetic cloth from Apollo spacesuits. Annals of Microbiology, 53, 47-54.

Brockett, R. M., and Ferguson, J. K. (1975). Microbiological sampling of the spacecraft atmosphere during a simulated skylab mission. Aviation, Space, and Environmental Medicine, 46(1), 30-32.

Brown, G. D., Denning, D. W., Gow, N. A. R., Levitz, S. M., Netea, M. G., and White, T. C. (2012). Hidden killers: human fungal infections. Science Translational Medicine, 4(165), 165 rv13. https://doi.org/10.1126/scitrans/med.3004404

Brown, N. A., and Goldman, G. H. (2016). The contribution of Aspergillus fumigatus stress responses to virulence and antifungal resistance. Journal of Microbiology, 54(3), 243-253. https://doi.org/10.1007/s12275-016-5510-4

Brungs, S., Kolanus, W., and Hemmersbach, R. (2015). Syk phosphorylation - a gravisensitive step in macrophage signalling. Cell Communication and Signaling : CCS, 13, 9. https://doi.org/10.1186/s12964-015-0088-8

Bucker, H., and Horneck, G. (1975). The biological effectiveness of HZE-particles of cosmic radiation studied in the Apollo 16 and 17 Biostack experiments. Acta Astronautica, 2(3-4), 247-264. https://doi.org/10.1016/0094-5765(75)90095-8

Caggiano, G., Napoli, C., Coretti, C., Lovero, G., Scarafile, G., De Giglio, O., and Montagna, M. T. (2014). Mold contamination in a controlled hospital environment: a 3-year surveillance in southern Italy. BMC Infectious Diseases, 14, 595. https://doi.org/10.1186/s12879-014-0595-z

Cairns, T. C., Nai, C., and Meyer, V. (2018). How a fungus shapes biotechnology: 100 years of Aspergillus niger research. Fungal Biology and Biotechnology, 5(1), 13. https://doi.org/10.1186/s40694-0180054-5

Cairns, T. C., Zheng, X., Zheng, P., Sun, J., and Meyer, V. (2019). Moulding the mould: understanding and reprogramming filamentous fungal growth and morphogenesis for next generation cell factories. Biotechnology for Biofuels, 12(1), 77. https://doi.org/10.1186/s13068-019-1400-4

Casadevall, A., Cordero, R. J. B., Bryan, R., Nosanchuk, J., and Dadachova, E. (2017). Melanin, radiation, and energy transduction in fungi. Microbiology Spectrum, 5(2). https://doi.org/10.1128/microbiolspec.FUNK-0037-2016

Castro, V. A., Thrasher, A. N., Healy, M., Ott, C. M., and Pierson, D. L. (2004). Microbial characterization during the early habitation of the International Space Station. Microbial Ecology, 47(2), 119-126. https://doi.org/10.1007/s00248-003-1030-y

Chancellor, J. C., Scott, G. B. I., and Sutton, J. P. (2014). Space Radiation: The number one risk to astronaut health beyond Low Earth Orbit. Life (Basel, Switzerland), 4(3), 491-510. https://doi.org/10.3390/life4030491

Checinska, A., Probst, A. J., Vaishampayan, P., White, J. R., Kumar, D., Stepanov, V. G., Fox, G. E., Nilsson, H. R., Pierson, D. L., Perry, J., and Venkateswaran, K. (2015). Microbiomes of the dust particles collected from the International Space Station and Spacecraft Assembly Facilities. Microbiome, 3, 50. https://doi.org/10.1186/s40168-015-0116-3 
Checinska Sielaff, A., Urbaniak, C., Mohan, G. B. M., Stepanov, V. G., Tran, Q., Wood, J. M., Minich, J., McDonald, D., Mayer, T., Knight, R., Karouia, F., Fox, G. E., and Venkateswaran, K. (2019). Characterization of the total and viable bacterial and fungal communities associated with the International Space Station surfaces. Microbiome, 7(1), 50. https://doi.org/10.1186/s40168-0190666-x

Cockell, C. S. (2010). Geomicrobiology beyond Earth: microbe-mineral interactions in space exploration and settlement. Trends in Microbiology, 18(7), 308-314. https://doi.org/10.1016/j.tim.2010.03.005

Cockell, C. S., Santomartino, R., Finster, K., Waajen, A. C., Eades, L. J., Moeller, R., Rettberg, P., Fuchs, F. M., Van Houdt, R., Leys, N., Coninx, I., Hatton, J., Parmitano, L., Krause, J., Koehler, A., Caplin, N., Zuijderduijn, L., Mariani, A., Pellari, S. S., ... Demets, R. (2020). Space station biomining experiment demonstrates rare earth element extraction in microgravity and Mars gravity. Nature Communications, 11(1), 5523. https://doi.org/10.1038/s41467-020-19276-w

Coetser, S. E., and Cloete, T. E. (2005). Biofouling and biocorrosion in industrial water systems. Critical Reviews in Microbiology, 31(4), 213-232. https://doi.org/10.1080/10408410500304074

Cogoli, A. (1993). The effect of hypogravity and hypergravity on cells of the immune system. Journal of Leukocyte Biology, 54(3), 259-268. https://doi.org/10.1002/jlb.54.3.259

Collivignarelli, M. C., Abbà, A., Miino, M. C., Caccamo, F. M., Torretta, V., Rada, E. C., and Sorlini, S. (2020). Disinfection of wastewater by UV-based treatment for reuse in a circular economy perspective. Where are we at? International Journal of Environmental Research and Public Health, 18(1). https://doi.org/10.3390/ijerph18010077

Conley, C. A. (2014). COSPAR BT - Encyclopedia of Astrobiology (R. Amils, M. Gargaud, J. Cernicharo Quintanilla, H. J. Cleaves, W. M. Irvine, D. Pinti, and M. Viso (Eds.); p. 1). Springer Berlin Heidelberg. https://doi.org/10.1007/978-3-642-27833-4_364-5

Cordero, R. J., and Casadevall, A. (2017). Functions of fungal melanin beyond virulence. Fungal Biology Reviews, 31(2), 99-112. https://doi.org/10.1016/j.fbr.2016.12.003

Corrêa, T. Q., Blanco, K. C., Inada, N. M., Hortenci, M. de F., Costa, A. A., Silva, E. da S., Gimenes, P. P. da C., Pompeu, S., de Holanda e Silva, R. L., Figueiredo, W. M., and Bagnato, V. S. (2017). Manual operated Ultraviolet surface decontamination for healthcare environments. Photomedicine and Laser Surgery, 35(12), 666-671. https://doi.org/10.1089/pho.2017.4298

Cortesão, M., Fuchs, F. M., Commichau, F. M., Eichenberger, P., Schuerger, A. C., Nicholson, W. L., Setlow, P., and Moeller, R. (2019). Bacillus subtilis spore resistance to simulated mars surface conditions. Frontiers in Microbiology, 10, 333. https://doi.org/10.3389/fmicb.2019.00333

Cortesão, M., Schütze, T., Marx, R., Moeller, R., and Meyer, V. (2020). Fungal Biotechnology in Space: Why and How? - Grand Challenges in Fungal Biotechnology (H. Nevalainen (Ed.); pp. 501-535). Springer International Publishing. https://doi.org/10.1007/978-3-030-29541-7_18

COSPAR. (2020). COSPAR Policy on Planetary Protection. Space Research Today, 208(June), 10-22. https://doi.org/10.1016/j.srt.2020.07.009

Crucian, B. E., Choukèr, A., Simpson, R. J., Mehta, S., Marshall, G., Smith, S. M., Zwart, S. R., Heer, M., Ponomarev, S., Whitmire, A., Frippiat, J. P., Douglas, G. L., Lorenzi, H., Buchheim, J.-I., Makedonas, G., Ginsburg, G. S., Ott, C. M., Pierson, D. L., Krieger, S. S., ... Sams, C. (2018). Immune system dysregulation during spaceflight: potential countermeasures for deep space exploration missions. Frontiers in Immunology, 9, 1437. https://doi.org/10.3389/fimmu.2018.01437 
Cucinotta, F. A., and Durante, M. (2006). Cancer risk from exposure to galactic cosmic rays: implications for space exploration by human beings. The Lancet Oncology, 7(5), 431-435. https://doi.org/https://doi.org/10.1016/S1470-2045(06)70695-7

Cucinotta, F. A., Hu, S., Schwadron, N. A., Kozarev, K., Townsend, L. W., and Kim, M.-H. Y. (2010). Space radiation risk limits and Earth-Moon-Mars environmental models. Space Weather, 8(12). https://doi.org/https://doi.org/10.1029/2010SW000572

Currie, J. N. (1917). The citric acid fermentation of Aspergillus niger. Journal of Biological Chemistry, 31(1), 15-37. https://doi.org/https://doi.org/10.1016/S0021-9258(18)86708-4

Dachev, T. P., Bankov, N. G., Tomov, B. T., Matviichuk, Y. N., Dimitrov, P. G., Häder, D.-P., and Horneck, G. (2017). Overview of the ISS Radiation Environment Observed during the ESA EXPOSE-R2 Mission in 2014-2016. Space Weather, 15(11), 1475-1489. https://doi.org/https://doi.org/10.1002/2016SW001580

Dadachova, E., and Casadevall, A. (2008). lonizing radiation: how fungi cope, adapt, and exploit with the help of melanin. Current Opinion in Microbiology, 11(6), 525-531. https://doi.org/10.1016/j.mib.2008.09.013

Damveld, R. A., Arentshorst, M., Franken, A., vanKuyk, P. A., Klis, F. M., van den Hondel, C. A. M. J. J., and Ram, A. F. J. (2005). The Aspergillus niger MADS-box transcription factor RImA is required for cell wall reinforcement in response to cell wall stress. Molecular Microbiology, 58(1), 305-319. https://doi.org/10.1111/j.1365-2958.2005.04827.x

de Castro, P. A., Colabardini, A. C., Manfiolli, A. O., Chiaratto, J., Silva, L. P., Mattos, E. C., Palmisano, G., Almeida, F., Persinoti, G. F., Ries, L. N. A., Mellado, L., Rocha, M. C., Bromley, M., Silva, R. N., de Souza, G. S., Loures, F. V., Malavazi, I., Brown, N. A., and Goldman, G. H. (2019). Aspergillus fumigatus calcium-responsive transcription factors regulate cell wall architecture promoting stress tolerance, virulence and caspofungin resistance. PLoS Genetics, 15(12), e1008551. https://doi.org/10.1371/journal.pgen.1008551

de Lima Alves, F., Stevenson, A., Baxter, E., Gillion, J. L. M., Hejazi, F., Hayes, S., Morrison, I. E. G., Prior, B. A., McGenity, T. J., Rangel, D. E. N., Magan, N., Timmis, K. N., and Hallsworth, J. E. (2015). Concomitant osmotic and chaotropicity-induced stresses in Aspergillus wentii: compatible solutes determine the biotic window. Current Genetics, 61(3), 457-477. https://doi.org/10.1007/s00294015-0496-8

De Middeleer, G., Leys, N., Sas, B., and De Saeger, S. (2019). Fungi and mycotoxins in space - a review. Astrobiology, 19(7), 915-926. https://doi.org/10.1089/ast.2018.1854

de Vera, J.-P., Alawi, M., Backhaus, T., Baqué, M., Billi, D., Böttger, U., Berger, T., Bohmeier, M., Cockell, C., Demets, R., de la Torre Noetzel, R., Edwards, H., Elsaesser, A., Fagliarone, C., Fiedler, A., Foing, B., Foucher, F., Fritz, J., Hanke, F., ... Zucconi, L. (2019). Limits of life and the habitability of Mars: the ESA space experiment BIOMEX on the ISS. Astrobiology, 19(2), 145-157. https://doi.org/10.1089/ast.2018.1897

Denning, D. W. (2017). Calling upon all public health mycologists. European Journal of Clinical Microbiology and Infectious Diseases, 36(6), 923-924. https://doi.org/10.1007/s10096-017-2909-8

Des Marais, D. J., Nuth, J. A. 3rd, Allamandola, L. J., Boss, A. P., Farmer, J. D., Hoehler, T. M., Jakosky, B. M., Meadows, V. S., Pohorille, A., Runnegar, B., and Spormann, A. M. (2008). The NASA Astrobiology roadmap. Astrobiology, 8(4), 715-730. https://doi.org/10.1089/ast.2008.0819 
Dijksterhuis, J., Nijsse, J., Hoekstra, F. A., and Golovina, E. A. (2007). High viscosity and anisotropy characterize the cytoplasm of fungal dormant stress-resistant spores. Eukaryotic Cell, 6(2), 157170. https://doi.org/10.1128/EC.00247-06

Dose, K., Bieger-Dose, A., Ernst, B., Feister, U., Gómez-Silva, B., Klein, A., Risi, S., and Stridde, C. (2001). Survival of microorganisms under the extreme conditions of the Atacama Desert. Origins of Life and Evolution of the Biosphere: The Journal of the International Society for the Study of the Origin of Life, 31(3), 287-303. https://doi.org/10.1023/a:1010788829265

Duran, R., Cary, J. W., and Calvo, A. M. (2010). Role of the osmotic stress regulatory pathway in morphogenesis and secondary metabolism in filamentous fungi. Toxins, 2(4), 367-381. https://doi.org/10.3390/toxins2040367

Eisenman, H. C., and Casadevall, A. (2012). Synthesis and assembly of fungal melanin. Applied Microbiology and Biotechnology, 93(3), 931-940. https://doi.org/10.1007/s00253-011-3777-2

ESA. (2010). Environment Control and life Support System (ECLSS). 1-4. http://erasmus.spaceflight.esa.int, accessed 6.05.2021

Esbelin, J., Mallea, S., Ram, A. F., and Carlin, F. (2013). Role of pigmentation in protecting Aspergillus niger conidiospores against pulsed light radiation. Photochemistry and Photobiology, 89(3), 758761. https://doi.org/10.1111/php.12037

Facius, R., Bucker, H., Horneck, G., Reitz, G., and Schafer, M. (1979). Dosimetric and biological results from the Bacillus subtilis Biostack experiment with the Apollo-Soyuz Test Project. Life Sciences and Space Research, 17, 123-128. https://doi.org/10.1016/b978-0-08-023416-8.50020-9

Fairén, A. G., Parro, V., Schulze-Makuch, D., and Whyte, L. (2017). Searching for life on Mars before it is too late. Astrobiology, 17(10), 962-970. https://doi.org/10.1089/ast.2017.1703

Fiedler, M. R., Lorenz, A., Nitsche, B. M., van den Hondel, C. A., Ram, A. F., and Meyer, V. (2014). The capacity of Aspergillus niger to sense and respond to cell wall stress requires at least three transcription factors: RImA, MsnA and CrzA. Fungal Biology and Biotechnology, 1, 5. https://doi.org/10.1186/s40694-014-0005-8

Frawley, D., and Bayram, Ö. (2020). The pheromone response module, a mitogen-activated protein kinase pathway implicated in the regulation of fungal development, secondary metabolism and pathogenicity. Fungal Genetics and Biology, 144, 103469.

https://doi.org/https://doi.org/10.1016/j.fgb.2020.103469

Fuchs, B. B., and Mylonakis, E. (2009). Our paths might cross: the role of the fungal cell wall integrity pathway in stress response and cross talk with other stress response pathways. Eukaryotic Cell, 8(11), 1616-1625. https://doi.org/10.1128/EC.00193-09

Gabani, P., and Singh, O. V. (2013). Radiation-resistant extremophiles and their potential in biotechnology and therapeutics. Applied Microbiology and Biotechnology, 97(3), 993-1004. https://doi.org/10.1007/s00253-012-4642-7

Galli, A., and Losch, A. (2019). Beyond planetary protection: What is planetary sustainability and what are its implications for space research? Life Sciences in Space Research, 23, 3-9. https://doi.org/https://doi.org/10.1016/j.lssr.2019.02.005

Garcia-Rubio, R., de Oliveira, H. C., Rivera, J., and Trevijano-Contador, N. (2020). The fungal cell wall: Candida, Cryptococcus, and Aspergillus species. Frontiers in Microbiology, 10, 2993. 
https://doi.org/10.3389/fmicb.2019.02993

Garrett-Bakelman, F. E., Darshi, M., Green, S. J., Gur, R. C., Lin, L., Macias, B. R., McKenna, M. J., Meydan, C., Mishra, T., Nasrini, J., Piening, B. D., Rizzardi, L. F., Sharma, K., Siamwala, J. H., Taylor, L., Vitaterna, M. H., Afkarian, M., Afshinnekoo, E., Ahadi, S., ... and Turek, F. W. (2019). The NASA Twins Study: A multidimensional analysis of a year-long human spaceflight. Science, 364(6436), eaau8650. https://doi.org/10.1126/science.aau8650

Garschagen, L. S., Mancinelli, R. L., and Moeller, R. (2019). Introducing Vibrio natriegens as a Microbial Model Organism for Microgravity Research. Astrobiology, 19(10), 1211-1220. https://doi.org/10.1089/ast.2018.2010

Garshnek, V. (1994). The lunar environment as a fractional-gravity biological laboratory. Acta Astronautica, 33, 211-215. https://doi.org/10.1016/0094-5765(94)90127-9

Geib, E., and Brock, M. (2017). ATNT: an enhanced system for expression of polycistronic secondary metabolite gene clusters in Aspergillus niger. Fungal Biology and Biotechnology, 4(1), 13. https://doi.org/10.1186/s40694-017-0042-1

Goldman, G. H., and Kafer, E. (2004). Aspergillus nidulans as a model system to characterize the DNA damage response in eukaryotes. Fungal Genetics and Biology, 41(4), 428-442. https://doi.org/https://doi.org/10.1016/j.fgb.2003.12.001

Goldman, G. H., McGuire, S. L., and Harris, S. D. (2002). The DNA damage response in filamentous fungi. Fungal Genetics and Biology : FG and B, 35(3), 183-195. https://doi.org/10.1006/fgbi.2002.1344

Gomoiu, I, Chatzitheodoridis, E., Vadrucci, S., Walther, I., and Cojoc, R. (2016). Fungal spores viability on the International Space Station. Origins of Life and Evolution of Biospheres, 46(4), 403-418. https://doi.org/10.1007/s11084-016-9502-5

Gomoiu, loana, Chatzitheodoridis, E., Vadrucci, S., and Walther, I. (2013). The effect of spaceflight on growth of Ulocladium chartarum colonies on the international space station. PloS One, 8(4), e62130. https://doi.org/10.1371/journal.pone.0062130

Gonçalves, V. N., Cantrell, C. L., Wedge, D. E., Ferreira, M. C., Soares, M. A., Jacob, M. R., Oliveira, F. S., Galante, D., Rodrigues, F., Alves, T. M. A., Zani, C. L., Junior, P. A. S., Murta, S., Romanha, A. J., Barbosa, E. C., Kroon, E. G., Oliveira, J. G., Gomez-Silva, B., Galetovic, A., ... Rosa, L. H. (2016). Fungi associated with rocks of the Atacama Desert: taxonomy, distribution, diversity, ecology and bioprospection for bioactive compounds. Environmental Microbiology, 18(1), 232-245. https://doi.org/https://doi.org/10.1111/1462-2920.13005

Gonçalves, V. N., Oliveira, F. S., Carvalho, C. R., Schaefer, C. E. G. R., Rosa, C. A., and Rosa, L. H. (2017). Antarctic rocks from continental Antarctica as source of potential human opportunistic fungi. Extremophiles : Life under Extreme Conditions, 21(5), 851-860. https://doi.org/10.1007/s00792017-0947-x

Gonzales, A. A., and Stoker, C. R. (2016). An efficient approach for Mars sample return using emerging commercial capabilities. Acta Astronautica, 123, 16-25. https://doi.org/10.1016/j.actaastro.2016.02.013

Gradini, R., Chen, F., Tan, R., and Newlin, L. (2019). A summary on cutting edge advancements in sterilization and cleaning technologies in medical, food, and drug industries, and its applicability to spacecraft hardware. Life Sciences in Space Research, 23, 31-49. https://doi.org/https://doi.org/10.1016/j.Issr.2019.05.002 
GSI. (2021). GSI Helmholtzzentrum für Schwerionenforschung - Accelerators. https://www.gsi.de/en/researchaccelerators/accelerator facility accessed 6.05.2021

Gulimova, V., Proshchina, A., Kharlamova, A., Krivova, Y., Barabanov, V., Berdiev, R., Asadchikov, V., Buzmakov, A., Zolotov, D., and Saveliev, S. (2019). Reptiles in space missions: results and perspectives. International Journal of Molecular Sciences, 20(12). https://doi.org/10.3390/ijms20123019

Gutarowska, B. (2010). Metabolic activity of moulds as a factor of building materials biodegradation. Polish Journal of Microbiology, 59(2), 119-124.

Guzmán-Chávez, F., Zwahlen, R. D., Bovenberg, R. A. L., and Driessen, A. J. M. (2018). Engineering of the filamentous fungus Penicillium chrysogenum as cell factory for natural products. In Frontiers in Microbiology (Vol. 9, p. 2768). https://www.frontiersin.org/article/10.3389/fmicb.2018.02768

Häder, D.-P. (2019). On the way to Mars-flagellated algae in bioregenerative life support systems under microgravity conditions. Frontiers in Plant Science, 10, 1621. https://doi.org/10.3389/fpls.2019.01621

Hagiwara, D., Sakamoto, K., Abe, K., and Gomi, K. (2016). Signaling pathways for stress responses and adaptation in Aspergillus species: stress biology in the post-genomic era. Bioscience, Biotechnology, and Biochemistry, 80(9), 1667-1680. https://doi.org/10.1080/09168451.2016.1162085

Hahn, A., and Hock, B. (1999). Chromosome mechanics of fungi under spaceflight conditions--tetrad analysis of two-factor crosses between spore color mutants of Sordaria macrospora. FASEB Journal: Official Publication of the Federation of American Societies for Experimental Biology, 13 Suppl, S149-56. https://doi.org/10.1096/fasebj.13.9001.s149

Hahn, C., Hans, M., Hein, C., Mancinelli, R. L., Mücklich, F., Wirth, R., Rettberg, P., Hellweg, C. E., and Moeller, R. (2017). Pure and oxidized copper materials as potential antimicrobial surfaces for spaceflight activities. Astrobiology, 17(12), 1183-1191. https://doi.org/10.1089/ast.2016.1620

Haines, S. R., Bope, A., Horack, J. M., Meyer, M. E., and Dannemiller, K. C. (2019). Quantitative evaluation of bioaerosols in different particle size fractions in dust collected on the International Space Station (ISS). Applied Microbiology and Biotechnology, 103(18), 7767-7782. https://doi.org/10.1007/s00253-019-10053-4

Harrell, C. R., Djonov, V., Fellabaum, C., and Volarevic, V. (2018). Risks of using sterilization by gamma radiation: the other side of the coin. International Journal of Medical Sciences, 15(3), 274-279. https://doi.org/10.7150/ijms.22644

Hartmann, T., Sasse, C., Schedler, A., Hasenberg, M., Gunzer, M., and Krappmann, S. (2011). Shaping the fungal adaptome - Stress responses of Aspergillus fumigatus. International Journal of Medical Microbiology, 301(5), 408-416. https://doi.org/https://doi.org/10.1016/j.ijmm.2011.04.008

Hasanain, F., Guenther, K., Mullett, W. M., and Craven, E. (2014). Gamma Sterilization of Pharmaceuticals -A review of the irradiation of excipients, active pharmaceutical ingredients, and final drug product formulations. PDA Journal of Pharmaceutical Science and Technology, 68(2), 113 LP - 137. https://doi.org/10.5731/pdajpst.2014.00955

Hassler, D. M., Zeitlin, C., Wimmer-Schweingruber, R. F., Ehresmann, B., Rafkin, S., Eigenbrode, J. L., Brinza, D. E., Weigle, G., Böttcher, S., Böhm, E., Burmeister, S., Guo, J., Köhler, J., Martin, C., Reitz, G., Cucinotta, F. A., Kim, M.-H., Grinspoon, D., Bullock, M. A., ... Team, M. S. L. S. (2014). Mars' surface radiation environment measured with the Mars Science Laboratory's Curiosity rover. 
Science, 343(6169), 1244797. https://doi.org/10.1126/science.1244797

Hayyan, M., Hashim, M. A., and AINashef, I. M. (2016). superoxide ion: Generation and chemical implications. Chemical Reviews, 116(5), 3029-3085. https://doi.org/10.1021/acs.chemrev.5b00407

He, L., Wang, S., Cortesão, M., Wu, M., Moeller, R., Setlow, P., and Li, Y.-Q. (2018). Single-cell analysis reveals individual spore responses to simulated space vacuum. NPJ Microgravity, 4, 26. https://doi.org/10.1038/s41526-018-0059-7

He, Y., Wang, B., Chen, W., Cox, R. J., He, J., and Chen, F. (2018). Recent advances in reconstructing microbial secondary metabolites biosynthesis in Aspergillus spp. Biotechnology Advances, 36(3), 739-783. https://doi.org/10.1016/j.biotechadv.2018.02.001

Hellweg, C. E., and Baumstark-Khan, C. (2007). Getting ready for the manned mission to Mars: the astronauts' risk from space radiation. Die Naturwissenschaften, 94(7), 517-526. https://doi.org/10.1007/s00114-006-0204-0

Hemmersbach, R., Strauch, S. M., Seibt, D., and Schuber, M. (2006). Comparative studies on gravisensitive protists on ground (2D and 3D clinostats) and in microgravity. Microgravity - Science and Technology, 18(3), 257-259. https://doi.org/10.1007/BF02870423

Henkel, J., and Hock, B. (1991). Clinostatic rotation decreases crossover frequencies in the fungus Sordaria macrospora Auersw. Microgravity Science and Technology, 4(4), 267-272.

Herranz, R., Anken, R., Boonstra, J., Braun, M., Christianen, P. C. M., de Geest, M., Hauslage, J., Hilbig, R., Hill, R. J. A., Lebert, M., Medina, F. J., Vagt, N., Ullrich, O., van Loon, J. J. W. A., and Hemmersbach, R. (2012). Ground-based facilities for simulation of microgravity: Organism-specific recommendations for their use, and recommended terminology. Astrobiology, 13(1), 1-17. https://doi.org/10.1089/ast.2012.0876

Hoffmeister, D., and Keller, N. P. (2007). Natural products of filamentous fungi: enzymes, genes, and their regulation. Natural Product Reports, 24(2), 393-416. https://doi.org/10.1039/B603084J

Horneck, G, Bucker, H., and Reitz, G. (1994). Long-term survival of bacterial spores in space. Advances in Space Research: The Official Journal of the Committee on Space Research (COSPAR), 14(10), 41-45. https://doi.org/10.1016/0273-1177(94)90448-0

Horneck, G, Facius, R., Enge, W., Beaujean, R., and Bartholoma, K. P. (1974). Microbial studies in the Biostack experiment of the Apollo 16 mission: germination and outgrowth of single Bacillus subtilis spores hit by cosmic HZE particles. Life Sciences and Space Research, 12, 75-83. https://doi.org/10.1016/b978-0-08-021783-3.50014-3

Horneck, G, Facius, R., Reichert, M., Rettberg, P., Seboldt, W., Manzey, D., Comet, B., Maillet, A., Preiss, H., Schauer, L., Dussap, C. G., Poughon, L., Belyavin, A., Reitz, G., Baumstark-Khan, C., and Gerzer, R. (2003). HUMEX, a study on the survivability and adaptation of humans to long-duration exploratory missions, part I: lunar missions. Advances in Space Research : The Official Journal of the Committee on Space Research (COSPAR), 31(11), 2389-2401. https://doi.org/10.1016/s02731177(03)00568-4

Horneck, Gerda, Baumstark-Khan, C., and Facius, R. (2006). Radiation Biology - Fundamentals of Space Biology: Research on Cells, Animals, and Plants in Space (G. Clément and K. Slenzka (Eds.); pp. 291336). Springer New York. https://doi.org/10.1007/0-387-37940-1_7

Horneck, Gerda, Klaus, D. M., and Mancinelli, R. L. (2010). Space microbiology. Microbiology and 
Molecular Biology Reviews : MMBR, 74(1), 121-156. https://doi.org/10.1128/MMBR.00016-09

Horneck, Gerda, Stöffler, D., Ott, S., Hornemann, U., Cockell, C. S., Moeller, R., Meyer, C., de Vera, J.-P., Fritz, J., Schade, S., and Artemieva, N. A. (2008). Microbial rock inhabitants survive hypervelocity impacts on Mars-like host planets: first phase of lithopanspermia experimentally tested. Astrobiology, 8(1), 17-44. https://doi.org/10.1089/ast.2007.0134

Horneck, Gerda, Walter, N., Westall, F., Grenfell, J. L., Martin, W. F., Gomez, F., Leuko, S., Lee, N., Onofri, S., Tsiganis, K., Saladino, R., Pilat-Lohinger, E., Palomba, E., Harrison, J., Rull, F., Muller, C., Strazzulla, G., Brucato, J. R., Rettberg, P., and Capria, M. T. (2016). AstRoMap European Astrobiology Roadmap. Astrobiology, 16(3), 201-243. https://doi.org/10.1089/ast.2015.1441

Huang, B., Li, D.-G., Huang, Y., and Liu, C.-T. (2018). Effects of spaceflight and simulated microgravity on microbial growth and secondary metabolism. Military Medical Research, 5(1), 18. https://doi.org/10.1186/s40779-018-0162-9

Ichijo, T., Yamaguchi, N., Tanigaki, F., Shirakawa, M., and Nasu, M. (2016). Four-year bacterial monitoring in the International Space Station-Japanese Experiment Module "Kibo" with culture-independent approach. NPJ Microgravity, 2, 16007. https://doi.org/10.1038/npjmgrav.2016.7

Ilgrande, C., Mastroleo, F., Christiaens, M. E. R., Lindeboom, R. E. F., Prat, D., Van Hoey, O., Ambrozova, I., Coninx, I., Heylen, W., Pommerening-Roser, A., Spieck, E., Boon, N., Vlaeminck, S. E., Leys, N., and Clauwaert, P. (2019). Reactivation of microbial strains and synthetic communities after a spaceflight to the International Space Station: Corroborating the Feasibility of Essential Conversions in the MELiSSA Loop. Astrobiology, 19(9), 1167-1176. https://doi.org/10.1089/ast.2018.1973

Inoue, H. (1999). DNA repair and specific-locus mutagenesis in Neurospora crassa. Mutation Research/Reviews in Mutation Research, 437(2), 121-133. https://doi.org/https://doi.org/10.1016/S1383-5742(99)00079-4

Inoue, H. (2011). Exploring the processes of DNA repair and homologous integration in Neurospora. Mutation Research/Reviews in Mutation Research, 728(1), 1-11. https://doi.org/https://doi.org/10.1016/j.mrrev.2011.06.003

Jensen, L. L., Merrison, J., Hansen, A. A., Mikkelsen, K. A., Kristoffersen, T., Nørnberg, P., Lomstein, B. A., and Finster, K. (2008). A facility for long-term Mars simulation experiments: the Mars Environmental Simulation Chamber (MESCH). Astrobiology, 8(3), 537-548. https://doi.org/10.1089/ast.2006.0092

Ji-Dong Gu, Roman, M., Esselman, T., and Mitchell, R. (1998). The role of microbial biofilms in deterioration of space station candidate materials. International Biodeterioration and Biodegradation, 41(1), 25-33. https://doi.org/https://doi.org/10.1016/S0964-8305(98)80005-X

Jiang, C., Guo, D., Li, Z., Lei, S., Shi, J., and Shao, D. (2019). Clinostat rotation affects metabolite transportation and increases organic acid production by Aspergillus carbonarius, as revealed by differential metabolomic analysis. Applied and Environmental Microbiology, 85(18), e01023-19. https://doi.org/10.1128/AEM.01023-19

Jiménez-Gómez, I., Valdés-Muñoz, G., Moreno-Perlin, T., Mouriño-Pérez, R. R., Sánchez-Carbente, M. D. R., Folch-Mallol, J. L., Pérez-Llano, Y., Gunde-Cimerman, N., Sánchez, N. D. C., and Batista-García, R. A. (2020). Haloadaptative responses of Aspergillus sydowii to extreme water deprivation: morphology, compatible solutes, and oxidative stress at $\mathrm{NaCl}$ saturation. Journal of Fungi (Basel, Switzerland), 6(4), 316. https://doi.org/10.3390/jof6040316 
Jørgensen, T. R., Park, J., Arentshorst, M., van Welzen, A. M., Lamers, G., vanKuyk, P. A., Damveld, R. A., van den Hondel, C. A. M., Nielsen, K. F., Frisvad, J. C., and Ram, A. F. J. (2011). The molecular and genetic basis of conidial pigmentation in Aspergillus niger. Fungal Genetics and Biology, 48(5), 544553. https://doi.org/10.1016/j.fgb.2011.01.005

Karouia, F., Peyvan, K., and Pohorille, A. (2017). Toward biotechnology in space: High-throughput instruments for in situ biological research beyond Earth. Biotechnology Advances, 35(7), 905-932. https://doi.org/10.1016/j.biotechadv.2017.04.003

Kerney, K. R., and Schuerger, A. C. (2011). Survival of Bacillus subtilis endospores on ultraviolet-irradiated rover wheels and Mars regolith under simulated Martian conditions. Astrobiology, 11(5), 477-485. https://doi.org/10.1089/ast.2011.0615

Kim, W., Tengra, F. K., Young, Z., Shong, J., Marchand, N., Chan, H. K., Pangule, R. C., Parra, M., Dordick, J. S., Plawsky, J. L., and Collins, C. H. (2013). Spaceflight promotes biofilm formation by Pseudomonas aeruginosa. PLOS ONE, 8(4), e62437. https://doi.org/10.1371/journal.pone.0062437

Kiss, J. Z. (2014). Plant biology in reduced gravity on the Moon and Mars. Plant Biology (Stuttgart, Germany), 16 Suppl 1, 12-17. https://doi.org/10.1111/plb.12031

Klaus, D. M. (2001). Clinostats and bioreactors. Gravitational and Space Biology Bulletin : Publication of the American Society for Gravitational and Space Biology, 14(2), 55-64.

Klintworth, R., Reher, H. J., Viktorov, A. N., and Bohle, D. (1999). Biological induced corrosion of materials II: new test methods and experiences from MIR station. Acta Astronautica, 44(7-12), 569-578. https://doi.org/10.1016/s0094-5765(99)00069-7

Knox, B. P., Blachowicz, A., Palmer, J. M., Romsdahl, J., Huttenlocher, A., Wang, C. C. C., Keller, N. P., and Venkateswaran, K. (2016). Characterization of Aspergillus fumigatus Isolates from Air and Surfaces of the International Space Station. MSphere, 1(5). https://doi.org/10.1128/mSphere.00227-16

Koga, S., Echigo, A., and Nunomura, K. (1966). Physical properties of cell water in partially dried Saccharomyces cerevisiae. Biophysical Journal, 6(5), 665-674. https://doi.org/10.1016/S00063495(66)86685-7

Kokilaramani, S., Al-Ansari, M. M., Rajasekar, A., Al-Khattaf, F. S., Hussain, A., and Govarthanan, M. (2021). Microbial influenced corrosion of processing industry by re-circulating waste water and its control measures - A review. Chemosphere, 265, 129075. https://doi.org/10.1016/j.chemosphere.2020.129075

Kopp, S., Krüger, M., Feldmann, S., Oltmann, H., Schütte, A., Schmitz, B., Bauer, J., Schulz, H., Saar, K., Huebner, N., Wehland, M., Nassef, M. Z., Melnik, D., Meltendorf, S., Infanger, M., and Grimm, D. (2018). Thyroid cancer cells in space during the TEXUS-53 sounding rocket mission - The THYROID Project. Scientific Reports, 8(1), 10355. https://doi.org/10.1038/s41598-018-28695-1

Koskinen, K., Rettberg, P., Pukall, R., Auerbach, A., Wink, L., Barczyk, S., Perras, A., Mahnert, A., Margheritis, D., Kminek, G., and Moissl-Eichinger, C. (2017). Microbial biodiversity assessment of the European Space Agency's ExoMars 2016 mission. Microbiome, 5(1), 143. https://doi.org/10.1186/s40168-017-0358-3

Lang, J. M., Coil, D. A., Neches, R. Y., Brown, W. E., Cavalier, D., Severance, M., Hampton-Marcell, J. T., Gilbert, J. A., and Eisen, J. A. (2017). A microbial survey of the International Space Station (ISS). PeerJ, 5, e4029. https://doi.org/10.7717/peerj.4029 
Latgé, J. P. (2017). 30 years of battling the cell wall. Medical Mycology, 55(1), 4-9. https://doi.org/10.1093/mmy/myw076

Lederberg, J. (1960). Exobiology: Approaches to life beyond the earth. Science (New York, N.Y.), 132(3424), 393-400. https://doi.org/10.1126/science.132.3424.393

Lee, G., and Arepally, G. M. (2012). Anticoagulation techniques in apheresis: from heparin to citrate and beyond. Journal of Clinical Apheresis, 27(3), 117-125. https://doi.org/10.1002/jca.21222

Leys, N. M. E. J., Hendrickx, L., De Boever, P., Baatout, S., and Mergeay, M. (n.d.). Spaceflight effects on bacterial physiology. Journal of Biological Regulators and Homeostatic Agents, 18(2), 193-199. Retrieved December 8, 2016, from http://www.ncbi.nlm.nih.gov/pubmed/15471227

Li, X., Cai, M., Wang, L., Niu, F., Yang, D., and Zhang, G. (2019). Evaluation survey of microbial disinfection methods in UV-LED water treatment systems. The Science of the Total Environment, 659, 14151427. https://doi.org/10.1016/j.scitotenv.2018.12.344

Limbach, C., Hauslage, J., Schäfer, C., and Braun, M. (2005). How to activate a plant gravireceptor. Early mechanisms of gravity sensing studied in characean rhizoids during parabolic flights. Plant Physiology, 139(2), 1030-1040. https://doi.org/10.1104/pp.105.068106

Liu, C., Zhong, G., Zhou, Y., Yang, Y., Tan, Y., Li, Y., Gao, X., Sun, W., Li, J., Jin, X., Cao, D., Yuan, X., Liu, Z., Liang, S., Li, Y., Du, R., Zhao, Y., Xue, J., Zhao, D., ... Li, Y. (2020). Alteration of calcium signalling in cardiomyocyte induced by simulated microgravity and hypergravity. Cell Proliferation, 53(3), e12783. https://doi.org/10.1111/cpr.12783

Longo, A., and Damer, B. (2020). Factoring origin of life hypotheses into the search for life in the solar system and beyond. Life (Basel, Switzerland), 10(5). https://doi.org/10.3390/life10050052

Lopez, J. V, Peixoto, R. S., and Rosado, A. S. (2019). Inevitable future: space colonization beyond Earth with microbes first. FEMS Microbiology Ecology, 95(10), fiz127. https://doi.org/10.1093/femsec/fiz127

Ma, L., Kazama, Y., Hirano, T., Morita, R., Tanaka, S., Abe, T., and Hatakeyama, S. (2018). LET dependence on killing effect and mutagenicity in the model filamentous fungus Neurospora crassa. International Journal of Radiation Biology, 94(12), 1125-1133. https://doi.org/10.1080/09553002.2019.1524940

Mahaffy, P. R., Webster, C. R., Atreya, S. K., Franz, H., Wong, M., Conrad, P. G., Harpold, D., Jones, J. J., Leshin, L. A., Manning, H., Owen, T., Pepin, R. O., Squyres, S., and Trainer, M. (2013). Abundance and isotopic composition of gases in the martian atmosphere from the Curiosity rover. Science, 341(6143), 263 LP - 266. https://doi.org/10.1126/science.1237966

Makimura, K., Hanazawa, R., Takatori, K., Tamura, Y., Fujisaki, R., Nishiyama, Y., Abe, S., Uchida, K., Kawamura, Y., Ezaki, T., and Yamaguchi, H. (2001). Fungal flora on board the Mir-Space Station, identification by morphological features and ribosomal DNA sequences. Microbiology and Immunology, 45(5), 357-363. https://doi.org/http://dx.doi.org/10.1111/j.13480421.2001.tb02631.x

Mann, V., Sundaresan, A., Mehta, S. K., Crucian, B., Doursout, M. F., and Devakottai, S. (2019). Effects of microgravity and other space stressors in immunosuppression and viral reactivation with potential nervous system involvement. Neurology India, 67(Supplement), S198-S203. https://doi.org/10.4103/0028-3886.259125

McIntyre, A. B. R., Rizzardi, L., Yu, A. M., Alexander, N., Rosen, G. L., Botkin, D. J., Stahl, S. E., John, K. K., 
Castro-Wallace, S. L., McGrath, K., Burton, A. S., Feinberg, A. P., and Mason, C. E. (2016). Nanopore sequencing in microgravity. NPJ Microgravity, 2, 16035. https://doi.org/10.1038/npjmgrav.2016.35

McNamara, C. J., Perry, T. D., Leard, R., Bearce, K., Dante, J., and Mitchell, R. (2005). Corrosion of aluminum alloy 2024 by microorganisms isolated from aircraft fuel tanks. Biofouling, 21(5-6), 257265. https://doi.org/10.1080/08927010500389921

McNulty, M. J., Xiong, Y. M., Yates, K., Karuppanan, K., Hilzinger, J. M., Berliner, A. J., Delzio, J., Arkin, A. P., Lane, N. E., Nandi, S., and McDonald, K. A. (2021). Molecular pharming to support human life on the moon, mars, and beyond. Critical Reviews in Biotechnology, 1-16. https://doi.org/10.1080/07388551.2021.1888070

Melloul, E., Luiggi, S., Anaïs, L., Arné, P., Costa, J.-M., Fihman, V., Briard, B., Dannaoui, E., Guillot, J., Decousser, J.-W., Beauvais, A., and Botterel, F. (2016). Characteristics of Aspergillus fumigatus in association with Stenotrophomonas maltophilia in an in vitro model of mixed biofilm. PloS One, 11(11), e0166325. https://doi.org/10.1371/journal.pone.0166325

Menezes, A. A., Cumbers, J., Hogan, J. A., and Arkin, A. P. (2015). Towards synthetic biological approaches to resource utilization on space missions. Journal of the Royal Society, Interface, 12(102), 20140715. https://doi.org/10.1098/rsif.2014.0715

Meyer, V., Arentshorst, M., El-Ghezal, A., Drews, A.-C., Kooistra, R., van den Hondel, C. A. M. J. J., and Ram, A. F. J. (2007). Highly efficient gene targeting in the Aspergillus niger kusA mutant. Journal of Biotechnology, 128(4), 770-775. https://doi.org/https://doi.org/10.1016/j.jbiotec.2006.12.021

Meyer, V., Basenko, E. Y., Benz, J. P., Braus, G. H., Caddick, M. X., Csukai, M., de Vries, R. P., Endy, D., Frisvad, J. C., Gunde-Cimerman, N., Haarmann, T., Hadar, Y., Hansen, K., Johnson, R. I., Keller, N. P., Kraševec, N., Mortensen, U. H., Perez, R., Ram, A. F. J., ... Wösten, H. A. B. (2020). Growing a circular economy with fungal biotechnology: a white paper. In Fungal biology and biotechnology (Vol. 7, p. 5). https://doi.org/10.1186/s40694-020-00095-z

Meyer, V., Wu, B., and Ram, A. F. J. (2011). Aspergillus as a multi-purpose cell factory: current status and perspectives. Biotechnology Letters, 33(3), 469-476. https://doi.org/10.1007/s10529-010-0473-8

Mhatre, S., Wood, J. M., Sielaff, A. C., Mora, M., Duller, S., Singh, N. K., Karouia, F., Moissl-Eichinger, C., and Venkateswaran, K. (2020). Assessing the risk of transfer of microorganisms at the International Space Station due to cargo delivery by commercial resupply vehicles. Frontiers in Microbiology, 11, 566412. https://doi.org/10.3389/fmicb.2020.566412

Misra, N. N., Yadav, B., Roopesh, M. S., and Jo, C. (2019). Cold plasma for effective fungal and mycotoxin control in foods: mechanisms, inactivation effects, and applications. Comprehensive Reviews in Food Science and Food Safety, 18(1), 106-120. https://doi.org/https://doi.org/10.1111/15414337.12398

Moeller, R., Horneck, G., Facius, R., and Stackebrandt, E. (2005). Role of pigmentation in protecting Bacillus sp. endospores against environmental UV radiation. FEMS Microbiology Ecology, 51(2), 231-236. https://doi.org/10.1016/j.femsec.2004.08.008

Moeller, R., Raguse, M., Reitz, G., Okayasu, R., Li, Z., Klein, S., Setlow, P., and Nicholson, W. L. (2014). Resistance of Bacillus subtilis spore DNA to lethal ionizing radiation damage relies primarily on spore core components and DNA repair, with minor effects of oxygen radical detoxification. Applied and Environmental Microbiology, 80(1), 104-109. https://doi.org/10.1128/AEM.03136-13

Moeller, R., Reitz, G., Berger, T., Okayasu, R., Nicholson, W. L., and Horneck, G. (2010)a. Astrobiological 
aspects of the mutagenesis of cosmic radiation on bacterial spores. Astrobiology, 10(5), 509-521. https://doi.org/10.1089/ast.2009.0429

Moeller, R., Rohde, M., and Reitz, G. (2010)b. Effects of ionizing radiation on the survival of bacterial spores in artificial martian regolith. Icarus, 206(2), 783-786. https://doi.org/https://doi.org/10.1016/j.icarus.2009.11.014

Moeller, R., Setlow, P., Horneck, G., Berger, T., Reitz, G., Rettberg, P., Doherty, A. J., Okayasu, R., and Nicholson, W. L. (2008). Roles of the major, small, acid-soluble spore proteins and spore-specific and universal DNA repair mechanisms in resistance of Bacillus subtilis spores to ionizing radiation from $X$ rays and high-energy charged-particle bombardment. Journal of Bacteriology, 190(3), 11341140. https://doi.org/10.1128/JB.01644-07

Moeller, R., Stackebrandt, E., Reitz, G., Berger, T., Rettberg, P., Doherty, A. J., Horneck, G., and Nicholson, W. L. (2007). Role of DNA repair by nonhomologous-end joining in Bacillus subtilis spore resistance to extreme dryness, mono- and polychromatic UV, and ionizing radiation. Journal of Bacteriology, 189(8), 3306-3311. https://doi.org/10.1128/JB.00018-07

Moissl-Eichinger, C., Rettberg, P., and Pukall, R. (2012). The first collection of spacecraft-associated microorganisms: a public source for extremotolerant microorganisms from spacecraft assembly clean rooms. Astrobiology, 12(11), 1024-1034. https://doi.org/10.1089/ast.2012.0906

Montibus, M., Pinson-Gadais, L., Richard-Forget, F., Barreau, C., and Ponts, N. (2015). Coupling of transcriptional response to oxidative stress and secondary metabolism regulation in filamentous fungi. Critical Reviews in Microbiology, 41(3), 295-308.

https://doi.org/10.3109/1040841X.2013.829416

Moosekian, S. R., Jeong, S., Marks, B. P., and Ryser, E. T. (2012). X-Ray irradiation as a microbial intervention strategy for food. Annual Review of Food Science and Technology, 3(1), 493-510. https://doi.org/10.1146/annurev-food-022811-101306

Mora, M., Mahnert, A., Koskinen, K., Pausan, M. R., Oberauner-Wappis, L., Krause, R., Perras, A. K., Gorkiewicz, G., Berg, G., and Moissl-Eichinger, C. (2016). Microorganisms in confined habitats: microbial monitoring and control of intensive care units, operating rooms, cleanrooms and the International Space Station. Frontiers in Microbiology, 7, 1573. https://doi.org/10.3389/fmicb.2016.01573

Motamedi, K., Colin, A. P., Hooijschuur, J. H., Postma, O., Lootens, R., Pruijser, D., Stoevelaar, R., Ariese, F., Hutchinson, I. B., Ingley, R., and Davies, G. R. (2015). Design of a Mars atmosphere simulation chamber and testing a Raman Laser Spectrometer (RLS) under conditions pertinent to Mars rover missions. EPJ Techniques and Instrumentation, 2(1), 15. https://doi.org/10.1140/epjti/s40485-0150025-7

Munir, M. T., and Federighi, M. (2020). Control of foodborne biological hazards by ionizing radiations. Foods (Basel, Switzerland), 9(7), 878. https://doi.org/10.3390/foods9070878

Najrana, T., and Sanchez-Esteban, J. (2016). Mechanotransduction as an Adaptation to Gravity. Frontiers in Pediatrics, 4, 140. https://doi.org/10.3389/fped.2016.00140

Nangle, S. N., Wolfson, M. Y., Hartsough, L., Ma, N. J., Mason, C. E., Merighi, M., Nathan, V., Silver, P. A., Simon, M., Swett, J., Thompson, D. B., and Ziesack, M. (2020). The case for biotech on Mars. Nature Biotechnology, 38(4), 401-407. https://doi.org/10.1038/s41587-020-0485-4

Nascimento, É., Da Silva, S. H., Dos Reis Marques, E., Roberts, D. W., and Braga, G. U. L. (2010). 
Quantification of cyclobutane pyrimidine dimers induced by uvb radiation in conidia of the fungi Aspergillus fumigatus, Aspergillus nidulans, Metarhizium acridum and Metarhizium robertsii. Photochemistry and Photobiology, 86(6), 1259-1266. https://doi.org/https://doi.org/10.1111/j.1751-1097.2010.00793.x

Newcombe, D. A., Schuerger, A. C., Benardini, J. N., Dickinson, D., Tanner, R., and Venkateswaran, K. (2005). Survival of spacecraft-associated microorganisms under simulated martian UV irradiation. Applied and Environmental Microbiology, 71(12), 8147-8156. https://doi.org/10.1128/AEM.71.12.8147-8156.2005

Newcombe, F. C. (1904). Limitations of the Klinostat as an instrument for scientific research. Science, 20(507), 376 LP - 379. https://doi.org/10.1126/science.20.507.376-b

Nicholson, W. L., Moeller, R., and Horneck, G. (2012). Transcriptomic responses of germinating Bacillus subtilis spores exposed to 1.5 years of space and simulated martian conditions on the EXPOSE-E experiment PROTECT. Astrobiology, 12(5), 469-486. https://doi.org/10.1089/ast.2011.0748

Nickerson, C. A., Ott, C. M., Wilson, J. W., Ramamurthy, R., and Pierson, D. L. (2004). Microbial responses to microgravity and other low-shear environments. Microbiology and Molecular Biology Reviews: MMBR, 68(2), 345-361. https://doi.org/10.1128/MMBR.68.2.345-361.2004

NIRS-HIMAC. (2021). Call for Proposal of experiments at "HIMAC." https://www.qst.go.jp/site/nirsenglish/20559.html accessed 6.05.2021

Novikova, N., De Boever, P., Poddubko, S., Deshevaya, E., Polikarpov, N., Rakova, N., Coninx, I., and Mergeay, M. (2006). Survey of environmental biocontamination on board the International Space Station. Research in Microbiology, 157(1), 5-12. https://doi.org/10.1016/j.resmic.2005.07.010

Onofri, S, Barreca, D., Selbmann, L., Isola, D., Rabbow, E., Horneck, G., de Vera, J. P. P., Hatton, J., and Zucconi, L. (2008). Resistance of Antarctic black fungi and cryptoendolithic communities to simulated space and martian conditions. Studies in Mycology, 61, 99-109. https://doi.org/10.3114/sim.2008.61.10

Onofri, Silvano, de la Torre, R., de Vera, J.-P., Ott, S., Zucconi, L., Selbmann, L., Scalzi, G., Venkateswaran, K. J., Rabbow, E., Sánchez Iñigo, F. J., and Horneck, G. (2012). Survival of rock-colonizing organisms after 1.5 years in outer space. Astrobiology, 12(5), 508-516. https://doi.org/10.1089/ast.2011.0736

Onofri, Silvano, Selbmann, L., Pacelli, C., de Vera, J. P., Horneck, G., Hallsworth, J. E., and Zucconi, L. (2018). Integrity of the DNA and cellular ultrastructure of cryptoendolithic fungi in space or Mars conditions: A 1.5-year study at the International Space Station. Life (Basel, Switzerland), 8(2). https://doi.org/10.3390/life8020023

Ott, C. M., Crabbé, A., Wilson, J. W., Barrila, J., Castro, S. L., and Nickerson, C. A. (2012). microbial stress: spaceflight-induced alterations in microbial virulence and infectious disease risks for the crew Stress challenges and immunity in space: From Mechanisms to Monitoring and Preventive Strategies (A. Chouker (Ed.); pp. 203-225). Springer Berlin Heidelberg. https://doi.org/10.1007/978-3-642-22272-6_15

Pacelli, C., Bryan, R. A., Onofri, S., Selbmann, L., Shuryak, I., and Dadachova, E. (2017). Melanin is effective in protecting fast and slow growing fungi from various types of ionizing radiation. Environmental Microbiology, 19(4), 1612-1624. https://doi.org/10.1111/1462-2920.13681

Pacelli, C., Bryan, R. A., Onofri, S., Selbmann, L., Zucconi, L., Shuryak, I., and Dadachova, E. (2018). The effect of protracted $X$-ray exposure on cell survival and metabolic activity of fast and slow growing 
fungi capable of melanogenesis. Environmental Microbiology Reports, 10(3), 255-263. https://doi.org/10.1111/1758-2229.12632

Pacelli, C., Cassaro, A., Aureli, L., Moeller, R., Fujimori, A., and Onofri, S. (2020). The responses of the black fungus Cryomyces antarcticus to high doses of accelerated helium ions radiation within martian regolith simulants and their relevance for Mars. Life (Basel, Switzerland), 10(8). https://doi.org/10.3390/life10080130

Pacelli, C., Selbmann, L., Zucconi, L., Coleine, C., de Vera, J.-P., Rabbow, E., Böttger, U., Dadachova, E., and Onofri, S. (2019). Responses of the black fungus Cryomyces antarcticus to simulated Mars and space conditions on rock analogs. Astrobiology, 19(2), 209-220. https://doi.org/10.1089/ast.2016.1631

Paris, S., Wysong, D., Debeaupuis, J.-P., Shibuya, K., Philippe, B., Diamond, R. D., and Latgé, J.-P. (2003). Catalases of Aspergillus fumigatus. Infection and Immunity, 71(6), 3551-3562. https://doi.org/10.1128/iai.71.6.3551-3562.2003

Patel, Z. S., Brunstetter, T. J., Tarver, W. J., Whitmire, A. M., Zwart, S. R., Smith, S. M., and Huff, J. L. (2020). Red risks for a journey to the red planet: The highest priority human health risks for a mission to Mars. NPJ Microgravity, 6(1), 33. https://doi.org/10.1038/s41526-020-00124-6

Paton, S., Moore, G., Campagnolo, L., and Pottage, T. (2020). Antimicrobial surfaces for use on inhabited spacecraft: A review. Life Sciences in Space Research, 26, 125-131. https://doi.org/10.1016/j.Issr.2020.05.004

Pel, H. J., de Winde, J. H., Archer, D. B., Dyer, P. S., Hofmann, G., Schaap, P. J., Turner, G., de Vries, R. P., Albang, R., Albermann, K., Andersen, M. R., Bendtsen, J. D., Benen, J. A. E., van den Berg, M., Breestraat, S., Caddick, M. X., Contreras, R., Cornell, M., Coutinho, P. M., ... Stam, H. (2007). Genome sequencing and analysis of the versatile cell factory Aspergillus niger CBS 513.88. Nature Biotechnology, 25(2), 221-231. https://doi.org/10.1038/nbt1282

Peleg, A. Y., Hogan, D. A., and Mylonakis, E. (2010). Medically important bacterial-fungal interactions. Nature Reviews Microbiology, 8(5), 340-349. https://doi.org/10.1038/nrmicro2313

Perchonok, M. H., Cooper, M. R., and Catauro, P. M. (2012). Mission to Mars: Food production and processing for the final frontier. Annual Review of Food Science and Technology, 3(1), 311-330. https://doi.org/10.1146/annurev-food-022811-101222

Perez-Cuesta, U., Aparicio-Fernandez, L., Guruceaga, X., Martin-Souto, L., Abad-Diaz-de-Cerio, A., Antoran, A., Buldain, I., Hernando, F. L., Ramirez-Garcia, A., and Rementeria, A. (2020). Melanin and pyomelanin in Aspergillus fumigatus: from its genetics to host interaction. International Microbiology, 23(1), 55-63. https://doi.org/10.1007/s10123-019-00078-0

Person, A. K., Chudgar, S. M., Norton, B. L., Tong, B. C., and Stout, J. E. (2010). Aspergillus niger: an unusual cause of invasive pulmonary aspergillosis. Journal of Medical Microbiology, 59(Pt 7), 834838. https://doi.org/10.1099/jmm.0.018309-0

Pierson, D. L. (2001). Microbial contamination of spacecraft. Gravitational and Space Biology Bulletin : Publication of the American Society for Gravitational and Space Biology, 14(2), 1-6.

Pitt, J. I., and Christian, J. H. (1970). Heat resistance of xerophilic fungi based on microscopical assessment of spore survival. Applied Microbiology, 20(5), 682-686. https://pubmed.ncbi.nlm.nih.gov/5485080 
Pletser, V. (2004). Short duration microgravity experiments in physical and life sciences during parabolic flights: the first 30 ESA campaigns. Acta Astronautica, 55(10), 829-854. https://doi.org/10.1016/j.actaastro.2004.04.006

Pombeiro-Sponchiado, S. R. (2017). Production of Melanin Pigment by Fungi and Its Biotechnological Applications (G. S. Sousa (Ed.); p. Ch. 4). IntechOpen. https://doi.org/10.5772/67375

Probst, A. J., and Vaishampayan, P. (2020). Are we there yet? understanding interplanetary microbial hitchhikers using molecular methods. Current Issues in Molecular Biology, 38, 33-52. https://doi.org/10.21775/cimb.038.033

PubMed-NCBI. (2021). PubMed NCBI entries for search using key-words "Aspergillus niger." https://pubmed.ncbi.nlm.nih.gov/?term=aspergillus+niger accessed 6.05.2021

Puleo, J. R., Oxborrow, G. S., Fields, N. D., Herring, C. M., and Smith, L. S. (1973). Microbiological profiles of four Apollo spacecraft. Applied Microbiology, 26(6), 838-845.

Punt, M., van den Brule, T., Teertstra, W. R., Dijksterhuis, J., den Besten, H. M. W., Ohm, R. A., and Wösten, H. A. B. (2020). Impact of maturation and growth temperature on cell-size distribution, heat-resistance, compatible solute composition and transcription profiles of Penicillium roqueforti conidia. Food Research International, 136, 109287. https://doi.org/https://doi.org/10.1016/j.foodres.2020.109287

Rabbow, E., Horneck, G., Rettberg, P., Schott, J.-U., Panitz, C., L'Afflitto, A., von Heise-Rotenburg, R., Willnecker, R., Baglioni, P., Hatton, J., Dettmann, J., Demets, R., and Reitz, G. (2009). EXPOSE, an astrobiological exposure facility on the international space station - from proposal to flight. Origins of Life and Evolution of the Biosphere: The Journal of the International Society for the Study of the Origin of Life, 39(6), 581-598. https://doi.org/10.1007/s11084-009-9173-6

Rabbow, E., Parpart, A., and Reitz, G. (2016). The planetary and space simulation facilities at DLR Cologne. Microgravity Science and Technology, 28(3), 215-229. https://doi.org/10.1007/s12217015-9448-7

Rabbow, E., Rettberg, P., Barczyk, S., Bohmeier, M., Parpart, A., Panitz, C., Horneck, G., Burfeindt, J., Molter, F., Jaramillo, E., Pereira, C., Weiß, P., Willnecker, R., Demets, R., Dettmann, J., and Reitz, G. (2015). The astrobiological mission EXPOSE-R on board of the International Space Station. International Journal of Astrobiology, 14(1), 3-16. https://doi.org/DOI: 10.1017/S1473550414000202

Rabbow, E., Rettberg, P., Parpart, A., Panitz, C., Schulte, W., Molter, F., Jaramillo, E., Demets, R., Weiß, P., and Willnecker, R. (2017). EXPOSE-R2: The astrobiological ESA mission on board of the International Space Station. Frontiers in Microbiology, 8, 1533. https://doi.org/10.3389/fmicb.2017.01533

Raddadi, N., Cherif, A., Daffonchio, D., Neifar, M., and Fava, F. (2015). Biotechnological applications of extremophiles, extremozymes and extremolytes. Applied Microbiology and Biotechnology, 99(19), 7907-7913. https://doi.org/10.1007/s00253-015-6874-9

Ramage, G., Rajendran, R., Gutierrez-Correa, M., Jones, B., and Williams, C. (2011). Aspergillus biofilms: clinical and industrial significance. FEMS Microbiology Letters, 324(2), 89-97. https://doi.org/10.1111/j.1574-6968.2011.02381.x

Ramage, G., Rajendran, R., Sherry, L., and Williams, C. (2012). Fungal biofilm resistance. International Journal of Microbiology, 2012, 528521. https://doi.org/10.1155/2012/528521 
Ravindran, R., and Jaiswal, A. K. (2019). Wholesomeness and safety aspects of irradiated foods. Food Chemistry, 285, 363-368. https://doi.org/https://doi.org/10.1016/j.foodchem.2019.02.002

Rcheulishvili, N., Zhang, Y., Papukashvili, D., and Deng, Y.-L. (2020). Survey and evaluation of spacecraftassociated aluminum-degrading microbes and their rapid identification methods. Astrobiology, 20(8), 925-934. https://doi.org/10.1089/ast.2019.2078

Regberg, A. B., Castro, C. L., Connolly, H. C. J., Davis, R. E., Dworkin, J. P., Lauretta, D. S., Messenger, S. R., Mclain, H. L., McCubbin, F. M., Moore, J. L., Righter, K., Stahl-Rommel, S., and Castro-Wallace, S. L. (2020). Prokaryotic and fungal characterization of the facilities used to assemble, test, and launch the OSIRIS-REx spacecraft. Frontiers in Microbiology, 11, 530661. https://doi.org/10.3389/fmicb.2020.530661

Rettberg, P, Rabbow, E., Panitz, C., and Horneck, G. (2004). Biological space experiments for the simulation of Martian conditions: UV radiation and martian soil analogues. Advances in Space Research : The Official Journal of the Committee on Space Research (COSPAR), 33(8), 1294-1301. https://doi.org/10.1016/j.asr.2003.09.050

Rettberg, Petra, Anesio, A. M., Baker, V. R., Baross, J. A., Cady, S. L., Detsis, E., Foreman, C. M., Hauber, E., Ori, G. G., Pearce, D. A., Renno, N. O., Ruvkun, G., Sattler, B., Saunders, M. P., Smith, D. H., Wagner, D., and Westall, F. (2016). Planetary protection and Mars Special Regions - A suggestion for updating the definition. Astrobiology, 16(2), 119-125. https://doi.org/10.1089/ast.2016.1472

Richardson, M., and Rautemaa-Richardson, R. (2019). Exposure to Aspergillus in home and healthcare facilities' water environments: Focus on biofilms. Microorganisms, 7(1), 7. https://doi.org/10.3390/microorganisms7010007

Romsdahl, J., Blachowicz, A., Chiang, A. J., Chiang, Y.-M., Masonjones, S., Yaegashi, J., Countryman, S., Karouia, F., Kalkum, M., Stajich, J. E., Venkateswaran, K., and Wang, C. C. C. (2019). International Space Station conditions alter genomics, proteomics, and metabolomics in Aspergillus nidulans. Applied Microbiology and Biotechnology, 103(3), 1363-1377. https://doi.org/10.1007/s00253-0189525-0

Romsdahl, J., Blachowicz, A., Chiang, A. J., Singh, N., Stajich, J. E., Kalkum, M., Venkateswaran, K., and Wang, C. C. C. (2018). Characterization of Aspergillus niger Isolated from the International Space Station. MSystems, 3(5). https://doi.org/10.1128/mSystems.00112-18

Romsdahl, J., Blachowicz, A., Chiang, Y.-M., Venkateswaran, K., and Wang, C. C. C. (2020). Metabolomic Analysis of Aspergillus niger isolated from the International Space Station reveals enhanced production levels of the antioxidant pyranonigrin A. Frontiers in Microbiology, 11, 931. https://doi.org/10.3389/fmicb.2020.00931

Rosenzweig, J. A., Abogunde, O., Thomas, K., Lawal, A., Nguyen, Y.-U., Sodipe, A., and Jejelowo, O. (2010). Spaceflight and modeled microgravity effects on microbial growth and virulence. Applied Microbiology and Biotechnology, 85(4), 885-891. https://doi.org/10.1007/s00253-009-2237-8

Rosenzweig, J. A., Ahmed, S., Eunson, J. J., and Chopra, A. K. (2014). Low-shear force associated with modeled microgravity and spaceflight does not similarly impact the virulence of notable bacterial pathogens. Applied Microbiology and Biotechnology, 98(21), 8797-8807. https://doi.org/10.1007/s00253-014-6025-8

Santiago, I. F., Gonçalves, V. N., Gómez-Silva, B., Galetovic, A., and Rosa, L. H. (2018). Fungal diversity in the Atacama Desert. Antonie van Leeuwenhoek, 111(8), 1345-1360. 
https://doi.org/10.1007/s10482-018-1060-6

Santomartino, R., Waajen, A. C., de Wit, W., Nicholson, N., Parmitano, L., Loudon, C.-M., Moeller, R., Rettberg, P., Fuchs, F. M., Van Houdt, R., Finster, K., Coninx, I., Krause, J., Koehler, A., Caplin, N., Zuijderduijn, L., Zolesi, V., Balsamo, M., Mariani, A., ... Cockell, C. S. (2020). No effect of microgravity and simulated Mars gravity on final bacterial cell concentrations on the International Space Station: Applications to Space Bioproduction. Frontiers in Microbiology, 11, 579156. https://doi.org/10.3389/fmicb.2020.579156

Santos, T. Dos, and de Castro, L. F. (2021). Evaluation of a portable Ultraviolet C (UV-C) device for hospital surface decontamination. Photodiagnosis and Photodynamic Therapy, 33, 102161. https://doi.org/10.1016/j.pdpdt.2020.102161

Sathishkumar, Y., Krishnaraj, C., Rajagopal, K., Sen, D., and Lee, Y. S. (2016). High throughput de novo RNA sequencing elucidates novel responses in Penicillium chrysogenum under microgravity. Bioprocess and Biosystems Engineering, 39(2), 223-231. https://doi.org/10.1007/s00449-015-15064

Sathishkumar, Y., Velmurugan, N., Lee, H. M., Rajagopal, K., Im, C. K., and Lee, Y. S. (2014). Effect of low shear modeled microgravity on phenotypic and central chitin metabolism in the filamentous fungi Aspergillus niger and Penicillium chrysogenum. Antonie van Leeuwenhoek, 106(2), 197-209. https://doi.org/10.1007/s10482-014-0181-9

Satoh, K., Yamazaki, T., Furukawa, S., Mukai, C., and Makimura, K. (2021). Identification of fungi isolated from astronaut nasal and pharyngeal smears and saliva. Microbiology and Immunology, 65(2), 8994. https://doi.org/10.1111/1348-0421.12872

Satoh, K., Yamazaki, T., Nakayama, T., Umeda, Y., Alshahni, M. M., Makimura, M., and Makimura, K. (2016). Characterization of fungi isolated from the equipment used in the International Space Station or Space Shuttle. Microbiology and Immunology, 60(5), 295-302. https://doi.org/https://doi.org/10.1111/1348-0421.12375

Schäpe, P., Kwon, M. J., Baumann, B., Gutschmann, B., Jung, S., Lenz, S., Nitsche, B., Paege, N., Schütze, T., Cairns, T. C., and Meyer, V. (2019). Updating genome annotation for the microbial cell factory Aspergillus niger using gene co-expression networks. Nucleic Acids Research, 47(2), 559-569. https://doi.org/10.1093/nar/gky1183

Schuerger, A. C., Mancinelli, R. L., Kern, R. G., Rothschild, L. J., and McKay, C. P. (2003). Survival of endospores of Bacillus subtilis on spacecraft surfaces under simulated martian environments: implications for the forward contamination of Mars. Icarus, 165(2), 253-276. https://doi.org/10.1016/s0019-1035(03)00200-8

Schuerger, A. C., Trigwell, S., and Calle, C. I. (2008). Use of non-thermal atmospheric plasmas to reduce the viability of Bacillus subtilis on spacecraft surfaces. International Journal of Astrobiology, 7, 4757. https://doi.org/10.1017/S1473550407004016

Schuetze, T., and Meyer, V. (2017). Polycistronic gene expression in Aspergillus niger. Microbial Cell Factories, 16(1), 162. https://doi.org/10.1186/s12934-017-0780-z

Schuster, E., Dunn-Coleman, N., Frisvad, J., and van Dijck, P. (2002). On the safety of Aspergillus niger - a review. Applied Microbiology and Biotechnology, 59(4), 426-435. https://doi.org/10.1007/s00253002-1032-6

Schwendner, P., Moissl-Eichinger, C., Barczyk, S., Bohmeier, M., Pukall, R., and Rettberg, P. (2013). 
Insights into the microbial diversity and bioburden in a South American spacecraft assembly clean room. Astrobiology, 13(12), 1140-1154. https://doi.org/10.1089/ast.2013.1023

Segers, F. J. J., Wösten, H. A. B., and Dijksterhuis, J. (2018). Aspergillus niger mutants affected in conidial pigmentation do not have an increased susceptibility to water stress during growth at low water activity. Letters in Applied Microbiology, 66(3), 238-243. https://doi.org/10.1111/lam.12846

Selbmann, L., Pacelli, C., Zucconi, L., Dadachova, E., Moeller, R., de Vera, J.-P., and Onofri, S. (2018). Resistance of an Antarctic cryptoendolithic black fungus to radiation gives new insights of astrobiological relevance. Fungal Biology, 122(6), 546-554. https://doi.org/https://doi.org/10.1016/j.funbio.2017.10.012

Senatore, G., Mastroleo, F., Leys, N., and Mauriello, G. (2018). Effect of microgravity and space radiation on microbes. Future Microbiology, 13, 831-847. https://doi.org/10.2217/fmb-2017-0251

Setlow, P. (2014). Spore resistance properties. Microbiology Spectrum, 2(5). https://doi.org/10.1128/microbiolspec.TBS-0003-2012

Sharma, M., Dubey, S., Darwhekar, G., and Jain, S. K. (2015). The diverse applications of plasma. AIP Conference Proceedings, 1670(1), 30027. https://doi.org/10.1063/1.4926711

Shin, K.-S., Park, H.-S., Kim, Y., Heo, I.-B., Kim, Y. H., and Yu, J.-H. (2016). Aspergillus fumigatus spore proteomics and genetics reveal that $\mathrm{VeA}$ represses DefA-mediated DNA damage response. Journal of Proteomics, 148, 26-35. https://doi.org/10.1016/j.jprot.2016.07.022

Silverman, G. J., Davis, N. S., and Beecher, N. (1967). Resistivity of spores to ultraviolet and gamma radiation while exposed to ultrahigh vacuum or at atmospheric pressure. Applied Microbiology, 15(3), 510-515.

Simões, M. F., and Antunes, A. (2021). Microbial pathogenicity in space. In Pathogens (Vol. 10, Issue 4). https://doi.org/10.3390/pathogens10040450

Singaravelan, N., Grishkan, I., Beharav, A., Wakamatsu, K., Ito, S., and Nevo, E. (2008). Adaptive melanin response of the soil fungus Aspergillus niger to UV radiation stress at "Evolution Canyon", Mount Carmel, Israel. PloS One, 3(8), e2993. https://doi.org/10.1371/journal.pone.0002993

Smith, D. J., and Sowa, M. B. (2017). Ballooning for biologists: Mission essentials for flying life science experiments to near space on NASA large scientific balloons. Gravitational and Space Research : Publication of the American Society for Gravitational and Space Research, 5(1), 52-73. https://pubmed.ncbi.nlm.nih.gov/31360738

Sobrado, J M, Martín-Soler, J., and Martín-Gago, J. A. (2014). Mimicking Mars: A vacuum simulation chamber for testing environmental instrumentation for Mars exploration. Review of Scientific Instruments, 85(3), 35111. https://doi.org/10.1063/1.4868592

Sobrado, Jesús Manuel. (2020). Mimicking the martian hydrological cycle: A set-up to introduce liquid water in vacuum. Sensors (Basel, Switzerland), 20(21), 6150. https://doi.org/10.3390/s20216150

Soccol, C., Vandenberghe, L., Rodrigues, C., and Pandey, A. (2006). New perspectives for citric acid production and application. Food Technology and Biotechnology, 44, 141-149.

Soffen, G. A. (1997). Astrobiology from exobiology: Viking and the current Mars probes. Acta Astronautica, 41(4-10), 609-611. https://doi.org/10.1016/s0094-5765(98)00055-1

Song, K., Mohseni, M., and Taghipour, F. (2016). Application of ultraviolet light-emitting diodes (UV-LEDs) 
for water disinfection: A review. Water Research, 94, 341-349.

https://doi.org/10.1016/j.watres.2016.03.003

Soreanu, G., Cretescu, I., Diaconu, M., Cojocaru, C., Ignat, M., Samoila, P., and Harabagiu, V. (2021). Investigation of a biosystem based on Arthrospira platensis for air revitalisation in spacecrafts: Performance evaluation through response surface methodology. Chemosphere, 264(Pt 2), 128465. https://doi.org/10.1016/j.chemosphere.2020.128465

SpaceX. (2021). Mars and beyond: The road to making humanity interplanetary. https://www.spacex.com/human-spaceflight/mars/

Stahl-Rommel, S., Jain, M., Nguyen, H. N., Arnold, R. R., Aunon-Chancellor, S. M., Sharp, G. M., Castro, C. L., John, K. K., Juul, S., Turner, D. J., Stoddart, D., Paten, B., Akeson, M., Burton, A. S., and CastroWallace, S. L. (2021). Real-time culture-independent microbial profiling onboard the International Space Station using nanopore sequencing. Genes, 12(1). https://doi.org/10.3390/genes12010106

Stenzel, C. (2016). Deployment of precise and robust sensors on board ISS-for scientific experiments and for operation of the station. Analytical and Bioanalytical Chemistry, 408(24), 6517-6536. https://doi.org/10.1007/s00216-016-9789-0

Stevenson, A., Hamill, P. G., O'Kane, C. J., Kminek, G., Rummel, J. D., Voytek, M. A., Dijksterhuis, J., and Hallsworth, J. E. (2017). Aspergillus penicillioides differentiation and cell division at 0.585 water activity. Environmental Microbiology, 19(2), 687-697. https://doi.org/https://doi.org/10.1111/1462-2920.13597

StiegImeier, M., Rettberg, P., Barczyk, S., Bohmeier, M., Pukall, R., Wirth, R., and Moissl-Eichinger, C. (2012). Abundance and diversity of microbial inhabitants in european spacecraft-associated clean rooms. Astrobiology, 12(6), 572-585. https://doi.org/10.1089/ast.2011.0735

Tesei, D., Chiang, A. J., Kalkum, M., Stajich, J. E., Mohan, G. B. M., Sterflinger, K., and Venkateswaran, K. (2021). Effects of simulated microgravity on the proteome and secretome of the polyextremotolerant black fungus Knufia chersonesos. Frontiers in Genetics, 12, 638708. https://doi.org/10.3389/fgene.2021.638708

Thammahong, A., Puttikamonkul, S., Perfect, J. R., Brennan, R. G., and Cramer, R. A. (2017). Central role of the trehalose biosynthesis pathway in the pathogenesis of human fungal infections: opportunities and challenges for therapeutic development. Microbiology and Molecular Biology Reviews : MMBR, 81(2), e00053-16. https://doi.org/10.1128/MMBR.00053-16

Thornhill, S. G., and Kumar, M. (2018). Biological filters and their use in potable water filtration systems in spaceflight conditions. Life Sciences in Space Research, 17, 40-43. https://doi.org/https://doi.org/10.1016/j.Issr.2018.03.003

Tong, Z., Zheng, X., Tong, Y., Shi, Y.-C., and Sun, J. (2019). Systems metabolic engineering for citric acid production by Aspergillus niger in the post-genomic era. Microbial Cell Factories, 18(1), 28. https://doi.org/10.1186/s12934-019-1064-6

Tribst, A. A. L., Franchi, M. A., Cristianini, M., and De Massaguer, P. R. (2009). Inactivation of Aspergillus niger in mango nectar by high-pressure homogenization combined with heat shock. Journal of Food Science, 74(9), M509-M514. https://doi.org/https://doi.org/10.1111/j.1750-3841.2009.01370.x

Turroni, S., Magnani, M., Kc, P., Lesnik, P., Vidal, H., and Heer, M. (2020). Gut microbiome and space travelers' health: State of the art and possible pro/prebiotic strategies for long-term space missions. Frontiers in Physiology, 11, 553929. https://doi.org/10.3389/fphys.2020.553929 
Urbaniak, C., van Dam, P., Zaborin, A., Zaborina, O., Gilbert, J. A., Torok, T., Wang, C. C. C., and Venkateswaran, K. (2019). Genomic characterization and virulence potential of two Fusarium oxysporum isolates cultured from the International Space Station. MSystems, 4(2). https://doi.org/10.1128/mSystems.00345-18

Vago, J. L., Westall, F., Coates, A. J., Jaumann, R., Korablev, O., Ciarletti, V., Mitrofanov, I., Josset, J.-L., De Sanctis, M. C., Bibring, J.-P., Rull, F., Goesmann, F., Steininger, H., Goetz, W., Brinckerhoff, W., Szopa, C., Raulin, F., Westall, F., Edwards, H. G. M., ... Carreau, C. (2017). Habitability on early Mars and the search for biosignatures with the ExoMars rover. Astrobiology, 17(6-7), 471-510. https://doi.org/10.1089/ast.2016.1533

Vaishampayan, A., and Grohmann, E. (2019). Multi-resistant biofilm-forming pathogens on the International Space Station. Journal of Biosciences, 44(5).

Vakkada Ramachandran, A., Nazarious, M. I., Mathanlal, T., Zorzano, M.-P., and Martín-Torres, J. (2020). Space environmental chamber for planetary studies. Sensors (Basel, Switzerland), 20(14), 3996. https://doi.org/10.3390/s20143996

Valero, A., Begum, M., Hocking, A. D., Marín, S., Ramos, A. J., and Sanchis, V. (2008). Mycelial growth and ochratoxin A production by Aspergillus section Nigri on simulated grape medium in modified atmospheres. Journal of Applied Microbiology, 105(2), 372-379. https://doi.org/10.1111/j.13652672.2008.03760.x

Valiante, V., Macheleidt, J., Föge, M., and Brakhage, A. A. (2015). The Aspergillus fumigatus cell wall integrity signaling pathway: drug target, compensatory pathways, and virulence. Frontiers in Microbiology, 6, 325. https://doi.org/10.3389/fmicb.2015.00325

van Loon, J. J., Folgering, E. H., Bouten, C. V, Veldhuijzen, J. P., and Smit, T. H. (2003). Inertial shear forces and the use of centrifuges in gravity research. What is the proper control? Journal of Biomechanical Engineering, 125(3), 342-346. https://doi.org/10.1115/1.1574521

van Loon, J. J. W. A. (2016). Centrifuges for microgravity simulation. The reduced gravity paradigm. In Frontiers in Astronomy and Space Sciences (Vol. 3, p. 21). https://www.frontiersin.org/article/10.3389/fspas.2016.00021

Van Mulders, S. E., Stassen, C., Daenen, L., Devreese, B., Siewers, V., van Eijsden, R. G. E., Nielsen, J., Delvaux, F. R., and Willaert, R. (2011). The influence of microgravity on invasive growth in Saccharomyces cerevisiae. Astrobiology, 11(1), 45-55. https://doi.org/10.1089/ast.2010.0518

Van Ombergen, A., Rossiter, A., and Ngo-Anh, T. J. (2021). 'White Mars' - nearly two decades of biomedical research at the Antarctic Concordia station. Experimental Physiology, 106(1), 6-17. https://doi.org/https://doi.org/10.1113/EP088352

Venkateswaran, K., Vaishampayan, P., Cisneros, J., Pierson, D. L., Rogers, S. O., and Perry, J. (2014). International Space Station environmental microbiome - microbial inventories of ISS filter debris. Applied Microbiology and Biotechnology, 98(14), 6453-6466. https://doi.org/10.1007/s00253-0145650-6

Videla, H. A., and Herrera, L. K. (2005). Microbiologically influenced corrosion: looking to the future. International Microbiology: The Official Journal of the Spanish Society for Microbiology, 8(3), 169180.

Wagner, I., Braun, M., Slenzka, K., and Posten, C. (2016). Photobioreactors in Life Support Systems. Advances in Biochemical Engineering/Biotechnology, 153, 143-184. 
https://doi.org/10.1007/10_2015_327

Walther, I. I., B, van der S., Boillat, M., and Cogoli, A. (2000). Performance of a miniaturized bioreactor in space flight: microtechnology at the service of space biology. Enzyme and Microbial Technology, 27(10), 778-783. https://doi.org/10.1016/s0141-0229(00)00300-8

Wang, H., Wang, X., Zhang, P., and Wang, Y. (2008). The Ku-dependent non-homologous end-joining but not other repair pathway is inhibited by high linear energy transfer ionizing radiation. DNA Repair, 7(5), 725-733. https://doi.org/https://doi.org/10.1016/j.dnarep.2008.01.010

Wassmann, M., Moeller, R., Reitz, G., and Rettberg, P. (2010). Adaptation of Bacillus subtilis cells to Archean-like UV climate: relevant hints of microbial evolution to remarkably increased radiation resistance. Astrobiology, 10(6), 605-615. https://doi.org/10.1089/ast.2009.0455

Witze, A. (2020). SpaceX to launch astronauts - and a new era of private human spaceflight. In Nature. https://doi.org/10.1038/d41586-020-01554-8

Wong, W. C., Dudinsky, L. A., Garcia, V. M., Ott, C. M., and Castro, V. A. (2010). Efficacy of various chemical disinfectants on biofilms formed in spacecraft potable water system components. Biofouling, 26(5), 583-586. https://doi.org/10.1080/08927014.2010.495772

Yamaguchi, N., Roberts, M., Castro, S., Oubre, C., Makimura, K., Leys, N., Grohmann, E., Sugita, T., Ichijo, T., and Nasu, M. (2014). Microbial monitoring of crewed habitats in space-current status and future perspectives. Microbes and Environments, 29(3), 250-260. https://doi.org/10.1264/jsme2.me14031

Yamazaki, T., Yoshimoto, M., Nishiyama, Y., Okubo, Y., and Makimura, K. (2012). Phenotypic characterization of Aspergillus niger and Candida albicans grown under simulated microgravity using a three-dimensional clinostat. Microbiology and Immunology, 56(7), 441-446. https://doi.org/https://doi.org/10.1111/j.1348-0421.2012.00471.x

Yang, S. S., Lin, J. Y., and Lin, Y. T. (1998). Microbiologically induced corrosion of aluminum alloys in fueloil/aqueous system. Journal of Microbiology, Immunology, and Infection = Wei Mian Yu Gan Ran Za Zhi, 31(3), 151-164. http://europepmc.org/abstract/MED/10496152

Zakharova, K., Marzban, G., de Vera, J.-P., Lorek, A., and Sterflinger, K. (2014). Protein patterns of black fungi under simulated Mars-like conditions. Scientific Reports, 4, 5114. https://doi.org/10.1038/srep05114

Zammuto, V., Fuchs, F. M., Fiebrandt, M., Stapelmann, K., Ulrich, N. J., Maugeri, T. L., Pukall, R., Gugliandolo, C., and Moeller, R. (2018). Comparing spore resistance of Bacillus strains isolated from hydrothermal vents and spacecraft assembly facilities to environmental stressors and decontamination treatments. Astrobiology, 18(11), 1425-1434. https://doi.org/10.1089/ast.2017.1715

Zea, L., Larsen, M., Estante, F., Qvortrup, K., Moeller, R., Dias de Oliveira, S., Stodieck, L., and Klaus, D. (2017). Phenotypic changes exhibited by E. coli cultured in space. Frontiers in Microbiology, 8, 1598. https://doi.org/10.3389/fmicb.2017.01598

Zea, L., McLean, R. J. C., Rook, T. A., Angle, G., Carter, D. L., Delegard, A., Denvir, A., Gerlach, R., Gorti, S., Mcllwaine, D., Nur, M., Peyton, B. M., Stewart, P. S., Sturman, P., and Velez Justiniano, Y. A. (2020). Potential biofilm control strategies for extended spaceflight missions. Biofilm, 2, 100026. https://doi.org/10.1016/j.bioflm.2020.100026 
Zeitlin, C., Hassler, D. M., Cucinotta, F. A., Ehresmann, B., Wimmer-Schweingruber, R. F., Brinza, D. E., Kang, S., Weigle, G., Böttcher, S., Böhm, E., Burmeister, S., Guo, J., Köhler, J., Martin, C., Posner, A., Rafkin, S., and Reitz, G. (2013). measurements of energetic particle radiation in transit to Mars on the Mars Science Laboratory. Science, 340(6136), 1080 LP - 1084.

https://doi.org/10.1126/science.1235989

Zhang, Y., Fan, J., Ye, J., and Lu, L. (2020). The fungal-specific histone acetyltransferase Rtt109 regulates development, DNA damage response, and virulence in Aspergillus fumigatus. Molecular Microbiology, n/a(n/a). https://doi.org/https://doi.org/10.1111/mmi.14665 


\section{Appendix}

Table 4: Abbreviation list

\section{Abbreviation}

2-D

3-D

A. niger

ATCC

BER

BIOMEX

CAB

CFW

COSPAR

CPD

CPDs

CWI

DDR

DHN

DLR

DNA

DSBs

DSM(Z)

ECM

ESA

GCR

GSI

HARV

HEPA

HOG

HR

HZE

ISS

ITS

L-DOPA

LEO

LET

LIFE

MAPK

MESCH

MSC

NASA

NER

NHEJ

\section{Connotation}

Two Dimensional

Three Dimentional

Aspergillus niger

American Type Culture Collection

Base Excision Repair

Biology and Mars Experiment

Center for Astrobiology

Calcofluor white

Committee on Space Research

Critical point drying

Cyclobutane pyrimidine dimers

Cell Wall Integrity

DNA Damage Response

1,8-dihydroxynaphthalene

German Aerospace Center

Deoxyribonucleic acid

Double-strand breaks

German Collection of Microorganisms

Extracellular matrix

European Space Agency

Galactic cosmic radiation

Hemholtz Center for Heavy Ion Research

High aspect rotating vessel

High efficiency particulate air filter

High Osmolality Glycerol

Homologous recombination

High energy charged particles

International Space Station

Internal transcribed spacer

3,4-dihydroxyphenylalanine

Low Earth Orbit

Linear Energy Transfer

Leading International Fungal Education

Mitogen-activated protein kinase

Mars Environmental Simulation Chamber

Mars Simulation Chamber

National Aeronautics and Space Administration

Nucleotide Excision repair

Non-Homologous End-Joining 


\begin{tabular}{ll} 
Abbreviation & Connotation \\
\hline OD & Optical density (nm) \\
P. rubens & Penicillium rubens \\
PBS & Phosphate buffered saline \\
PFA & Paraformaldehyde \\
pH & Potential of hydrogen: measure of acidity or alkalinity \\
PKS & Polyketide synthase \\
RNA & Ribonucleic acid \\
ROS & Reactive oxygen species \\
RPM & Random positioning machine (or 3-D Clinostat) \\
RWV & Rotating wall vessel \\
SEM & Scanning electron microscopy \\
SMG & Simulated microgravity \\
SSBs & Single-strand breaks \\
TEM & Transmission electron microscopy \\
UHV & Ultra-high vacuum \\
UV & Ultraviolet (radiation)
\end{tabular}

Table 5: Units and their description

\begin{tabular}{ll} 
Unit & Description \\
\hline $\mathbf{- g}$ & g-force \\
${ }^{\circ} \mathbf{C}$ & degrees Celsius \\
$\boldsymbol{\mu l}$ & microliter \\
$\boldsymbol{\mu m}$ & micrometer \\
$\mathbf{a w}$ & water activity \\
$\mathbf{b p}$ & Base pairs \\
$\mathbf{C F U}$ & colony forming unit \\
$\mathbf{C M}$ & Complete media \\
$\mathbf{g}$ & gram \\
$\mathbf{G y}$ & Gray \\
$\mathbf{h}$ & hour \\
$\mathbf{k b}$ & Kilobase pairs \\
$\mathbf{J}$ & Joule \\
$\mathbf{k e V}$ & kilo electronvolt \\
$\mathbf{L}$ & liter \\
Log & logaritmic \\
$\mathbf{m}$ & meter \\
$\mathbf{M}$ & Mol \\
MM & Minimal Media \\
$\mathbf{m i n}$ & minutes \\
& \\
\hline
\end{tabular}




\begin{tabular}{ll} 
Unit & Description \\
\hline $\mathbf{m L}$ & milliliter \\
$\mathbf{m M}$ & millimolar \\
$\mathbf{m m}$ & millimeter \\
$\mathbf{m W}$ & milliWatt \\
$\mathbf{n}$ & number of single experiments \\
$\mathbf{N m}$ & nanometer \\
$\mathbf{N}$ & Tested condition \\
$\mathbf{N O}$ & Control for the tested condition \\
$\mathbf{n g}$ & Nanogram \\
$\mathbf{n m}$ & nanometer \\
$\mathbf{R H}$ & Relative humidity [\%] \\
$\mathbf{r . p .} \mathbf{m}$. & rounds/revelations per minute \\
$\mathbf{T}$ & Temperature $\left(\mathbf{C}^{\circ}\right)$ \\
$\mathbf{W}$ & Watt \\
$\mathbf{Z}$ & Atomic number
\end{tabular}

